\title{
Concentrations of Glyphosate, Its Degradation Product, Aminomethylphosphonic Acid, and Glufosinate in Ground- and Surface-Water, Rainfall, and Soil Samples Collected in the United States, 2001-06
}

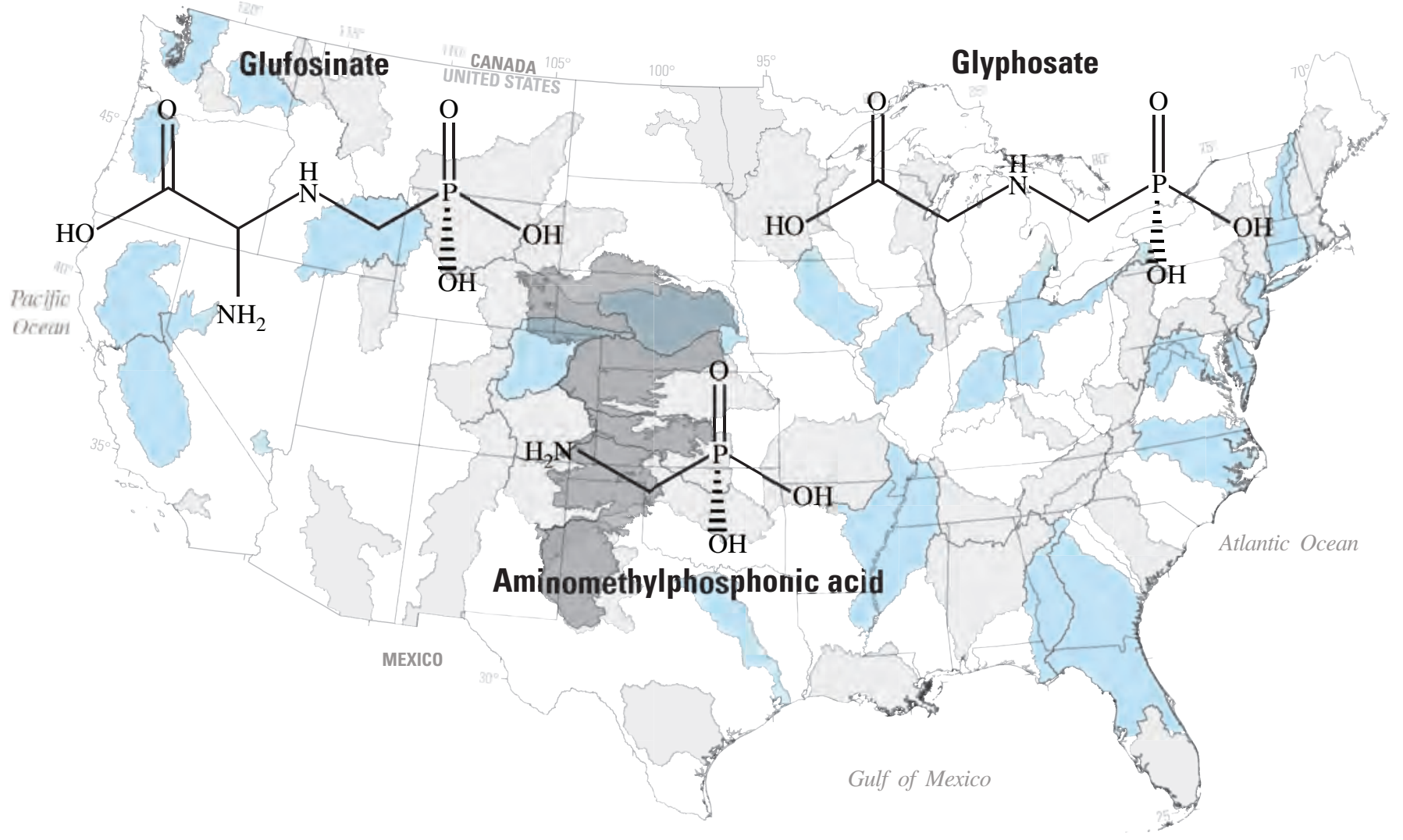

NAWQA Study Units

Scientific Investigations Report 2007-5122 



\section{Concentrations of Glyphosate, Its Degradation Product, Aminomethylphosphonic Acid, and Glufosinate in Ground- and Surface-Water, Rainfall, and Soil Samples Collected in the United States, 2001-06}

By Elisabeth A. Scribner, William A. Battaglin, Robert J. Gilliom, and Michael T. Meyer

Scientific Investigations Report 2007-5122 


\section{U.S. Department of the Interior DIRK KEMPTHORNE, Secretary}

\section{U.S. Geological Survey \\ Mark D. Myers, Director}

\section{U.S. Geological Survey, Reston, Virginia: 2007}

For product and ordering information:

World Wide Web: http://www.usgs.gov/pubprod

Telephone: 1-888-ASK-USGS

For more information on the USGS — the Federal source for science about the Earth, its natural and living resources, natural hazards, and the environment:

World Wide Web: http://www.usgs.gov

Telephone: 1-888-ASK-USGS

Any use of trade, product, or firm names is for descriptive purposes only and does not imply endorsement by the U.S. Government.

Although this report is in the public domain, permission must be secured from the individual copyright owners to reproduce any copyrighted materials contained within this report.

Suggested citation:

Scribner, E.A., Battaglin, W.A., Gilliom, R.J., and Meyer, M.T., 2007, Concentrations of glyphosate, its degradation product, aminomethylphosphonic acid, and glufosinate in ground- and surface-water, rainfall, and soil samples collected in the United States, 2001-06: U.S. Geological Survey Scientific Investigations Report 2007-5122, 111 p. 


\section{Contents}

Abstract

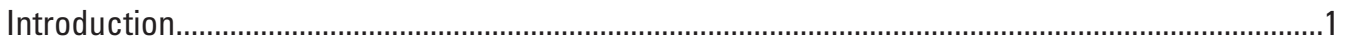

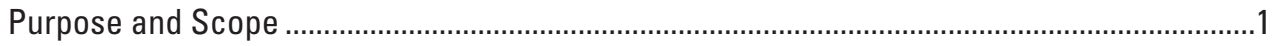

Description of Study Sites ...................................................................................................2

Description and Use of Glyphosate, Aminomethylphosphonic Acid, and Glufosinate .............2

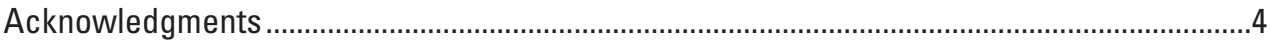

Sampling Methods and Procedures ..................................................................................

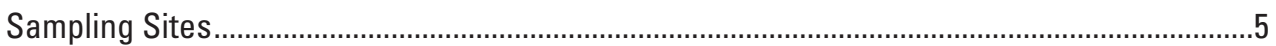

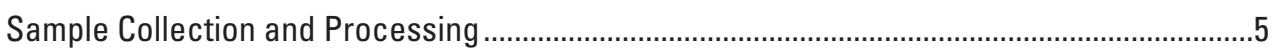

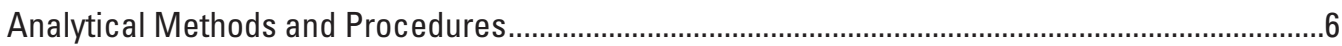

Liquid Chromatography/Mass Spectrometry and Liquid Chromatography/Tandem Mass

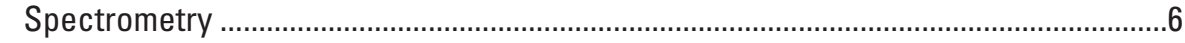

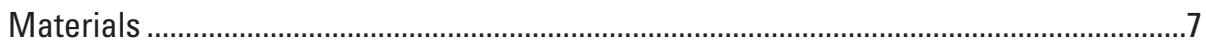

Derivatization of Samples........................................................................................

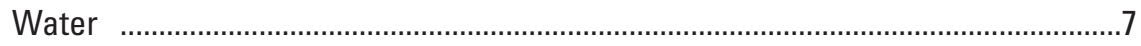

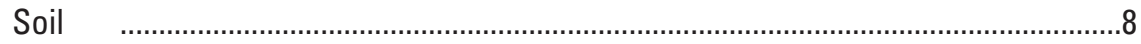

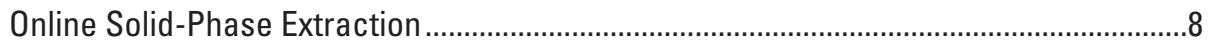

Liquid Chromatography/Mass Spectrometry .............................................................

Liquid Chromatography/Tandem Mass Spectrometry.................................................

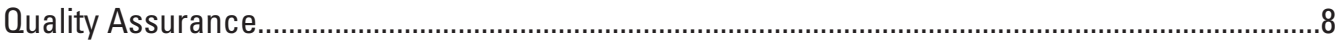

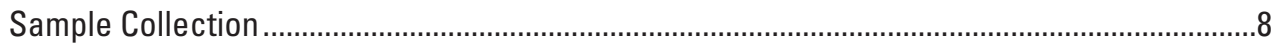

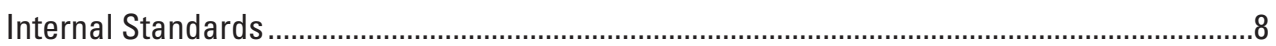

Laboratory Blank

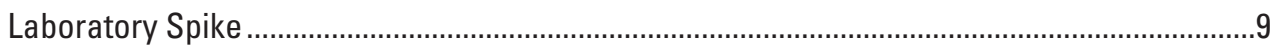

Instrumental Analysis Quality Control .................................................................................

Concentrations of Glyphosate, Aminomethylphosphonic Acid, and Glufosinate ..............................

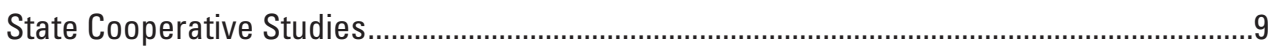

National Stream Quality Accounting Network (NASQAN) Program.......................................

National Water-Quality Assessment (NAWQA) Program .......................................................

Toxic Substances Hydrology (Toxics) Program .................................................................

Reconnaissance Data From 51 Streams in Nine Midwestern States............................12

Lower Mississippi River Basin .................................................................................12

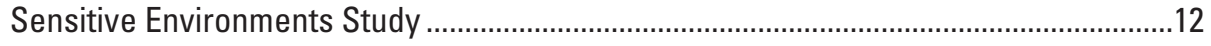

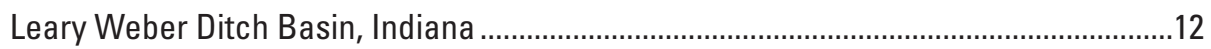

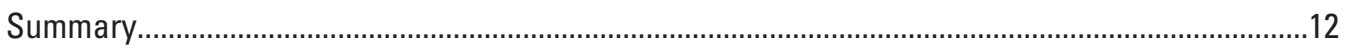

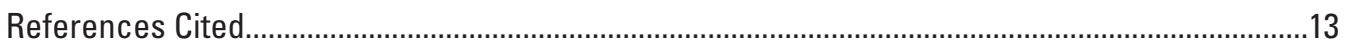

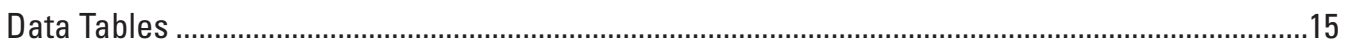

\section{Figures}

1. Chemical structures of glyphosate, its degradation product, aminomethylphosphonic acid, and glufosinate. 
2. Map showing location of 10 States where samples were collected for analysis of glyphosate, its degradation product, aminomethylphosphonic acid, and glufosinate, 2001-06

3. Map showing location of streamflow-gaging stations where samples were collected for analysis of glyphosate, its degradation product, aminomethylphosphonic acid, and glufosinate as part of the U.S. Geological Survey National Stream Quality Accounting Network Program, 2002

4. Map showing location of National Water-Quality Assessment Program study units where samples were collected for analysis of glyphosate, its degradation product, aminomethylphosphonic acid, and glufosinate, 2001-06.

5. Map showing location of Midwest Corn Belt and Toxic Substances Hydrology Program studies where samples were collected for analysis of glyphosate, its degradation product, aminomethylphosphonic acid, and glufosinate, 2001-06.

6. Graph showing glyphosate use in nine Midwestern States, 1989-2003

\section{Tables}

1. Example of analytical sequence for use in determining glyphosate, aminomethylphosphonic acid, and glufosinate in water samples

2. Summary of glyphosate, aminomethylphosphonic acid, and glufosinate concentrations analyzed by the U.S. Geological Survey Organic Geochemistry Research Laboratory, Lawrence, Kansas, for ground-water, surface-water, rainfall, and soil samples collected for State cooperative, National Stream Quality Accounting Network Program, National Water-Quality Assessment Program, and Toxic Substances Hydrology Program studies, 2001-06.

3. Analytical results for glyphosate, aminomethylphosphonic acid, and glufosinate in State cooperative surface- and ground-water studies, 2001-06

4. Analytical results for glyphosate, aminomethylphosphonic acid, and glufosinate in National Stream Quality Accounting Network Program surface- and ground-water studies, 2002

5. Analytical results for glyphosate, aminomethylphosphonic acid, and glufosinate in National Water-Quality Assessment Program surface- and ground-water studies, 2001-06

6. Analytical results for glyphosate, aminomethylphosphonic acid, and glufosinate in Toxic Substances Hydrology Program studies, 2001-06. 


\title{
Conversion Factors
}

\begin{tabular}{lcl}
\hline \multicolumn{1}{c}{ Multiply } & \multicolumn{1}{c}{ By } & \multicolumn{1}{c}{ To obtain } \\
\hline acre & 4,047 & square meter $\left(\mathrm{m}^{2}\right)$ \\
liter $(\mathrm{L})$ & 0.2642 & gallon $(\mathrm{gal})$ \\
microgram $(\mu \mathrm{g})$ & $3.527 \times 10^{-8}$ & ounce $(\mathrm{oz})$ \\
micrometer $(\mu \mathrm{m})$ & $3.937 \times 10^{-5}$ & inch $(\mathrm{in})$. \\
milligram $(\mathrm{mg})$ & $3.53 \times 10^{-5}$ & ounce $(\mathrm{oz})$ \\
pound $(\mathrm{lb})$ & 0.45359 & kilogram $(\mathrm{k})$ \\
pound per square inch $\left(\mathrm{lb} / \mathrm{in}^{2}\right)$ & 0.07031 & kilogram per square centimeter $\left(\mathrm{kg} / \mathrm{cm}^{2}\right)$ \\
square mile $\left(\mathrm{mi}^{2}\right)$ & 2.590 & square kilometer $\left(\mathrm{km}^{2}\right)$ \\
\hline
\end{tabular}

Temperature in degrees Celsius $\left({ }^{\circ} \mathrm{C}\right)$ may be converted to degrees Fahrenheit $\left({ }^{\circ} \mathrm{F}\right)$ as follows:

$$
{ }^{\circ} \mathrm{F}=\left(1.8 x^{\circ} \mathrm{C}\right)+32 .
$$

Temperature in degrees Fahrenheit $\left({ }^{\circ} \mathrm{F}\right)$ may be converted to degrees Celsius $\left({ }^{\circ} \mathrm{C}\right)$ as follows:

${ }^{\circ} \mathrm{C}=\left({ }^{\circ} \mathrm{F}-32\right) / 1.8$.

\section{Abbreviated Water-Quality Units}

\author{
gram (g) \\ microgram per kilogram $(\mu \mathrm{g} / \mathrm{kg})$ \\ microgram per liter $(\mu \mathrm{g} / \mathrm{L})$ \\ microgram per milliliter $(\mu \mathrm{g} / \mathrm{mL})$ \\ microliter $(\mu \mathrm{L})$ \\ milligram per liter (mg/L) \\ milliliter $(\mathrm{mL})$ \\ milliliter per minute $(\mathrm{mL} / \mathrm{min})$ \\ molar (M) \\ parts per billion (ppb)
}




\section{Other Abbreviations Used in This Report}

$\begin{array}{ll}\text { ACN } & \text { acetonitrile } \\ \text { AMPA } & \text { aminomethylphosphonic acid } \\ \text { AP } & \text { atmospheric pressure ionization } \\ \text { COB } & \text { carry-over blank } \\ \text { EDTA } & \text { ethylenediaminetetraacetic acid } \\ \text { FMOC } & \text { fluorenylmethylchloroformate } \\ \text { GC/MS } & \text { gas chromatography/mass spectrometry } \\ \text { HCl } & \text { hydrochloric acid } \\ \text { HPLC } & \text { high-performance liquid chromatography } \\ \text { KOH } & \text { potassium hydroxide } \\ \text { LC } & \text { liquid chromatography } \\ \text { LC/MS } & \text { liquid chromatography/mass spectrometry } \\ \text { LC/MS/MS } & \text { liquid chromatography/tandem mass spectrometry } \\ \text { MRL } & \text { minimum reporting level } \\ \text { NaOH } & \text { sodium hydroxide } \\ \text { NASQAN } & \text { National Stream Quality Accounting Network } \\ \text { NAWQA } & \text { National Water-Quality Assessment Program } \\ \text { OGRL } & \text { Organic Geochemistry Research Laboratory } \\ \text { SPE } & \text { Solid-phase extraction } \\ \text { Toxics } & \text { Toxic Substances Hydrology Program } \\ \text { USGS } & \text { U.S. Geological Survey }\end{array}$

\section{Definition}

Minimum reporting level (MRL)-smallest measured concentration of a constituent that may be reliably reported by using a given analytical method (Timme, 1995).

\section{Datum}

Horizontal coordinate information is referenced to the North American Datum of 1983 (NAD 83). 


\title{
Concentrations of Glyphosate, Its Degradation Product, Aminomethylphosphonic Acid, and Glufosinate in Ground- and Surface-Water, Rainfall, and Soil Samples Collected in the United States, 2001-06
}

\author{
By Elisabeth A. Scribner', William A. Battaglin², Robert J. Gilliom³, and Michael T. Meyer ${ }^{1}$
}

\section{Abstract}

The U.S. Geological Survey conducted a number of studies from 2001 through 2006 to investigate and document the occurrence, fate, and transport of glyphosate, its degradation product, aminomethylphosphonic acid (AMPA), and glufosinate in 2,135 ground- and surface-water samples, 14 rainfall samples, and 193 soil samples. Analytical methods were developed to detect and measure glyphosate, AMPA, and glufosinate in water, rainfall, and soil. Results show that AMPA was detected more frequently and occurred at similar or higher concentrations than the parent compound, glyphosate, whereas glufosinate was seldom found in the environment. Glyphosate and AMPA were detected more frequently in surface water than in ground water. Trace levels of glyphosate and AMPA may persist in the soil from year to year. The methods and data described in this report are useful to researchers and regulators interested in the occurrence, fate, and transport of glyphosate and AMPA in the environment.

\section{Introduction}

Nonpoint-source contamination of water resources from herbicides became a major water-quality concern during the 1990s in the United States. To address this issue, numerous studies of ground and surface water, rainfall, and soil were completed by the U.S. Geological Survey (USGS) (Scribner and others, 2005). The Organic Geochemistry Research Laboratory (OGRL) was established by the USGS in Lawrence, Kansas, to identify new or seldom-studied contaminants that may have deleterious effects on water quality or may be useful indicators of geochemical transport processes and to conduct

\footnotetext{
${ }^{1}$ U.S. Geological Survey, Lawrence, Kansas.

${ }^{2}$ U.S. Geological Survey, Lakewood, Colorado.

${ }^{3}$ U.S. Geological Survey, Sacramento, California.
}

research on the fate and transport of contaminants in the hydrogeologic system.

To accomplish this, analytical methods have been developed by OGRL using solid-phase extraction (SPE), gas chromatography/mass spectrometry (GC/MS), liquid chromatography/mass spectrometry (LC/MS), and liquid chromatography/tandem spectrometry (LC/MS/MS) to detect and measure pesticides, antibiotics, and their degradation products, including glyphosate, its degradation product, aminomethylphosphonic acid (AMPA), and glufosinate in ground and surface water, rainfall, and soil throughout the United States as identified in this report.

Glyphosate, often referred to as Roundup ${ }^{\mathrm{TM}}$, currently (2007) is the most commonly used agricultural herbicide in the United States and the world. Glyphosate herbicides have been popular since first marketed in 1974. In agriculture, glyphosate is used extensively with genetically modified herbicide-tolerant crops, but it also is commonly used around homes in yards, gardens, and other nonagricultural areas (Williams and others, 2000; Cox, 2004). In the past 10 years, it has become one of the most extensively used herbicides for weed control in corn and soybeans in the United States.

Glufosinate, a shortened name for glufosinate-ammonium salt, is a broad-spectrum, contact herbicide first introduced in the United States in 1997 and is used to control a wide range of weeds after the crop emerges or for total vegetation control. Glufosinate herbicides also are often used to desiccate crops before harvest (Friends of the Earth, 2001). Use of glufosinate is expected to increase substantially in the next few years due to the cultivation of crops, such as corn and soybeans, that have been genetically engineered to tolerate it (Cox, 1996).

\section{Purpose and Scope}

The purpose of this report is to present methods and data that document the occurrence and concentrations of glyphosate, its degradation product, AMPA, and glufosinate (chemical structures shown in fig. 1) in 873 ground- and 1,262 surface-water samples, 14 rainfall samples, and 193 soil 
Glyphosate<smiles>O=C(O)CNCP(=O)(O)O</smiles>

Aminomethylphosphonic acid (AMPA)

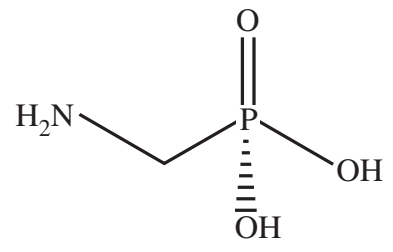

Glufosinate

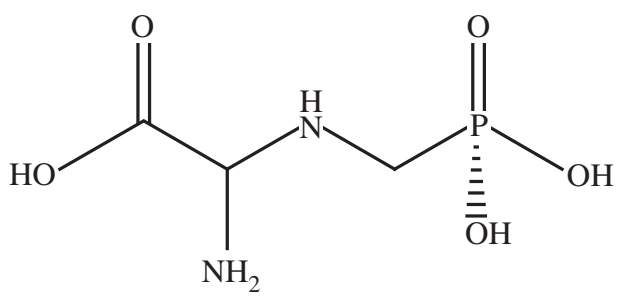

Figure 1. Chemical structures of glyphosate, its degradation product, aminomethylphosphonic acid, and glufosinate.

samples collected from 2001 through 2006. These samples were collected as part of several ongoing USGS studies being conducted throughout the United States, including studies conducted with Federal and State cooperative agencies and other USGS programs such as the National Stream Quality Accounting Network (NASQAN) Program, the National Water-Quality Assessment (NAWQA) Program, and the Toxic Substances Hydrology (Toxics) Program. The data in this report were collected from studies funded, in part or whole, by these programs. This report also includes a chemical description of glyphosate, AMPA, and glufosinate and documents the sample-collection and processing methods in addition to laboratory and analytical methods.

\section{Description of Study Sites}

Water samples were collected and analyzed as part of various USGS studies listed below.

1. USGS cooperative studies with various Federal and State agencies investigated glyphosate, AMPA, and glufosinate transport in aquatic ecosystems (fig. 2). In some cases, these cooperative studies evaluated agricultural contamination of ground and surface water from nonpoint and distributed point sources. For example, herbicide-related samples were collected in 2002 in cooperation with the U.S. Environmental Protection Agency to study urban contributions of glyphosate and AMPA to streams in the United States (Scribner and others, 2003).

2. The NASQAN program was established to develop a long-term water chemistry data set from five major river basins - the Colorado, Columbia, Mississippi, Rio Grande, and Yukon (fig. 3). In 2002, water samples from four streamflow-gaging stations in the Mississippi River Basin were collected for analysis (fig. 3) and results for glyphosate, AMPA, and glufosinate are reported herein. These data are important in characterizing baseline hydrology conditions and variations in chemical and sediment concentrations that occur throughout the year, during different seasons, during periods of high and low streamflow, and under different conditions of land use (Hooper and others, 1997; Coupe and Goolsby, 1999; Hooper and others, 2001; U.S. Geological Survey, 2006a).

3. NAWQA includes investigations of 51 major river basins and aquifer system study units (fig. 4) that provide the framework for national and regional status and trends in the quality of the Nation's ground- and surface-water resources (U.S. Geological Survey, 2003; Gilliom and Hamilton, 2006; Gilliom and others, 2006). Data for glyphosate, AMPA, and glufosinate from 23 of the 51 study units are included in this report.

4. The USGS Toxic Substances Hydrology (Toxics) Program, Pesticide Research Group, provides unbiased scientific information on the occurrence, movement, flux, fate, and effects of agricultural chemicals in the Nation's hydrologic environments. The program includes development and quantification of methods to measure contaminants and their degradation products at concentrations low enough to understand fate and transport in hydrologic systems (Buxton, 2000). An example of studies funded by Toxics includes herbicide and degradation product concentrations evidenced during a reconnaissance of 51 streams in nine Midwestern States in the Midwest Corn Belt during 2002 (fig. 5; Scribner and others, 2003; Battaglin and others, 2005; Kolpin and others, 2006).

\section{Description and Use of Glyphosate, Aminomethylphosphonic Acid, and Glufosinate}

Glyphosate [N-(phosphonomethyl)-glycine] is a nonselective, postemergence herbicide used for perennial weed control. Glyphosate was developed by J.E. Franz, Monsanto Corp., in 1971 and was released commercially in 1974 (Iowa State University, 1997). In the early 1980s, it became the first 


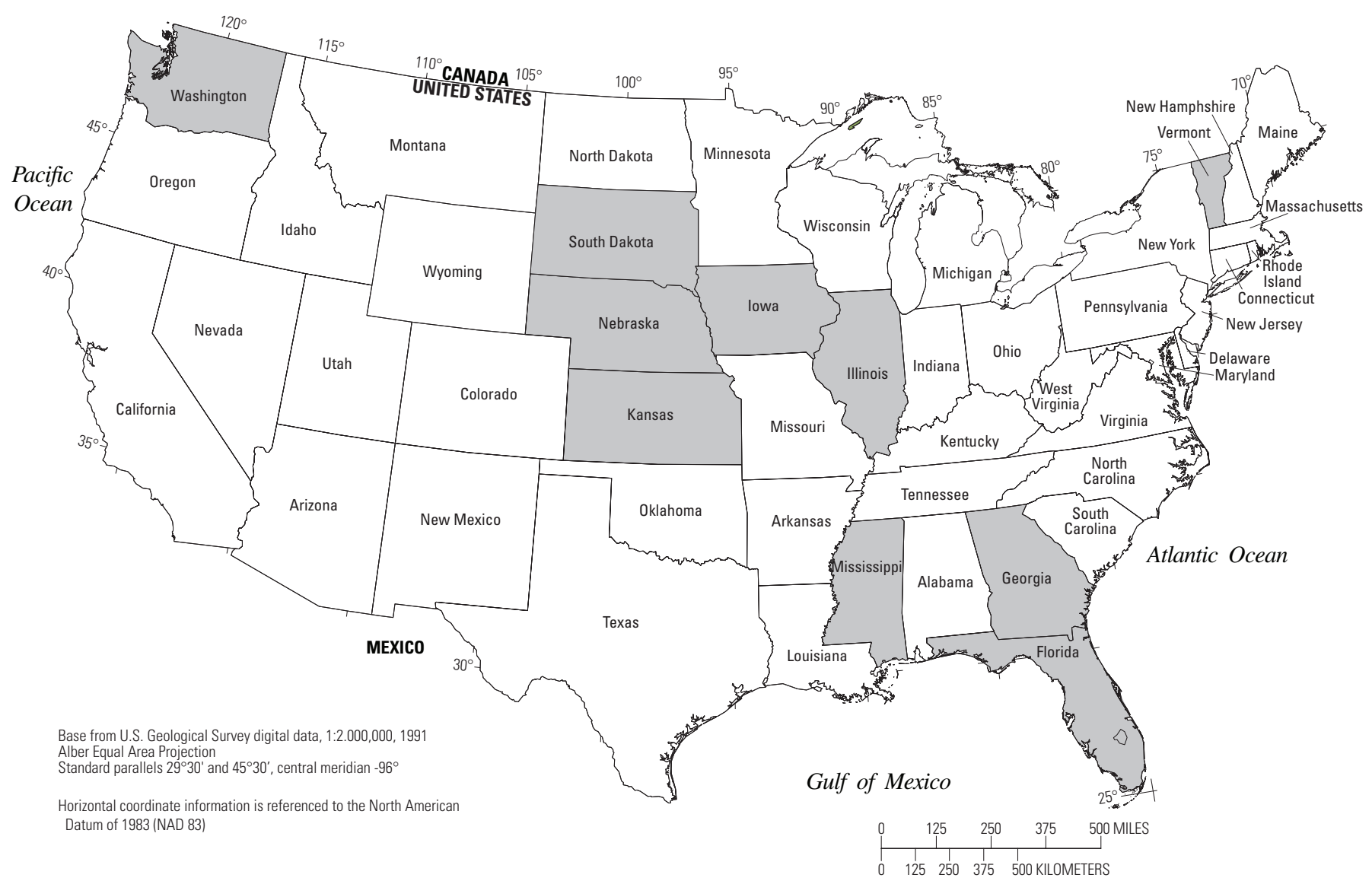

EXPLANATION

State where samples were collected-Results are shown in table 3

Figure 2. Ten States where samples were collected for analysis of glyphosate, its degradation product, aminomethylphosphonic acid, and glufosinate, 2001-06.

individual pesticide with worldwide sales of over 1 billion dollars (Iowa State University, 1997). Glyphosate use in the United States rapidly increased with the introduction of genetically modified glyphosate-resistant soybeans in 1997 and corn in 1998. Glyphosate also is used extensively in yards, gardens, parks, and other nonagricultural areas (Cox, 2004). Glyphosate was the world's most extensively used herbicide in 2000 (Baylis, 2000). In the United States, estimated usage is between 103 and 113 million lbs (Cox, 2004; Kiely and others, 2004). Herbicide use is an important factor affecting the concentrations of herbicides measured in Midwestern streams (Battaglin and Goolsby, 1999; Scribner and others, 2000; Battaglin and others, 2005). About 55 million lbs of glyphosate were applied to agricultural land in nine Midwestern States (fig. 6) during 2003 compared to atrazine, which decreased to about 40 million lbs during the same timeframe (Scribner and others, 2005; U.S. Department of Agriculture, 2006).

Glyphosate is absorbed directly through plant leaves and rapidly spreads throughout the plant. It blocks the activity of an enzyme used by plants to make amino acids. Without these amino acids, the plant cannot make proteins required for various life processes, resulting in the death of the plant (Monsanto Company, 2002; Cox, 2004). Glyphosate is known to bind tightly to particles that are adsorbed to soils that may prevent excessive leaching or being taken up from the soil by nontarget plants (Carlisle and Trevors, 1988). Glyphosate is degraded primarily by microbial processes producing AMPA [alpha-amino-3-hydroxy-5-methyl-4-isoxazolepropionic acid] (Sprankle and others, 1975; Rueppel and others, 1977). Studies show that glyphosate has a water solubility of 12,000 to $900,000 \mathrm{mg} / \mathrm{L}$ at $20^{\circ} \mathrm{C}$ and that the half-life can vary from 2 to 174 days (Extension Toxicology Network, 1994).

Glufosinate [2-amino-4-(hydroxy-methylphosphinyl) butanoic acid], a broad-spectrum contact herbicide used to control a wide range of weeds after the crop emerges or for total vegetation control on land not used for cultivation, is produced by AgrEvo, a joint venture by the German chemical corporations, Hoechst and Schering. The herbicide was first introduced in Japan and the United Kingdom in 1984. Registration in the United States was in 1993. Glufosinate is 


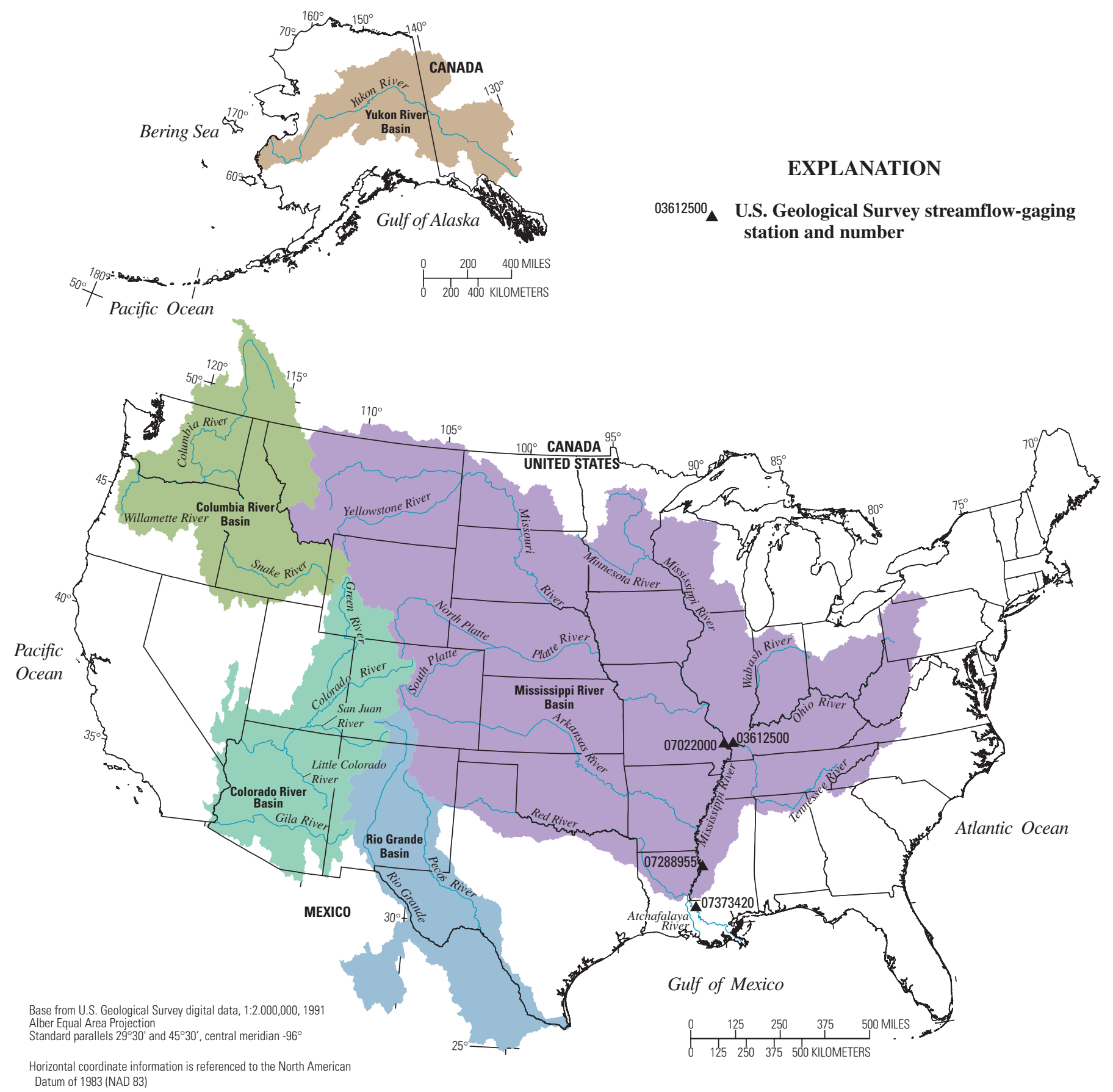

Figure 3. Location of streamflow-gaging stations where samples were collected for analysis of glyphosate, its degradation product, aminomethylphosphonic acid, and glufosinate as part of the U.S. Geological Survey National Stream Quality Accounting Network Program, 2002.

registered for use in more than 40 countries. AgrEvo introduced a glufosinate formulation for use on crops resistant to glufosinate in the late 1990s (Pesticide Action Network UK, 1998).

Studies show that $1,370 \mathrm{~g}$ of glufosinate can be dissolved in $1 \mathrm{~L}$ of water and the half-life will vary from 12 to 70 days, with an average of about 40 days (Cox, 1996). Usage of glufosinate in the United States was 847,000 lbs during 2003 (U.S. Department of Agriculture, 2006) and included nine
States (California, Iowa, Michigan, Minnesota, North Dakota, Oregon, South Dakota, Washington, and Wisconsin).

\section{Acknowledgments}

The authors wish to acknowledge the USGS scientists and hydrologic technicians who provided essential support to the studies just described by identifying candidate stream 


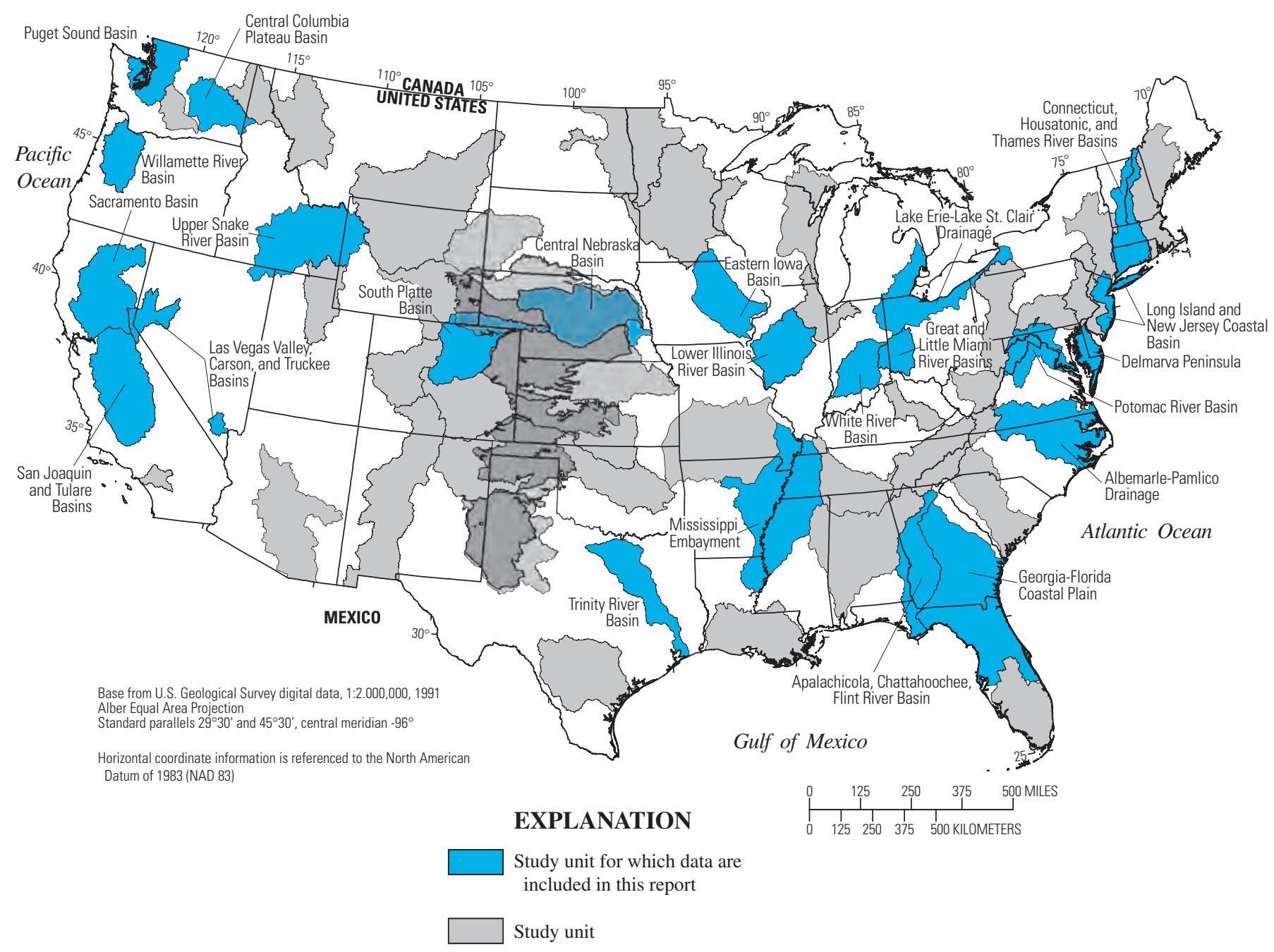

Figure 4. Location of National Water-Quality Assessment Program study units where samples were collected for analysis of glyphosate, its degradation product, aminomethylphosphonic acid, and glufosinate, 2001-06.

sites across the United States and in collecting and processing stream samples. In addition, thanks go to E. Michael Thurman (retired USGS research scientist) and Edward A. Lee (former OGRL staff member) for their important contributions in developing and interpreting the analytical methods used on samples described in this report.

\section{Sampling Methods and Procedures}

\section{Sampling Sites}

Drainage basins for surface-water sampling sites ranged in size from just a few acres to one of the largest river basins in the world (the Mississippi) and everywhere in-between. The data presented in this report are not meant to be a representative examination of the occurrence of glyphosate in the Nation's water but rather are a compilation of individual studies conducted for various purposes and with different objectives. The interpretation of these data as a whole should reflect this fact. However, these data do give an indication of the breadth and scope of the occurrence of glyphosate in the Nation's water from 2001-06.

\section{Sample Collection and Processing}

Hydrologic technicians collected water samples that accurately represent the water-quality characteristics of the ground water, surface water, rainfall, or soil at a given time or location following instructions of the USGS National Field Manual for Collection of Water-Quality Data (U.S. Geological Survey, 2006b). Sample-collection equipment was made of Teflon or stainless-steel material and was free of materials that might leach interfering compounds into water samples or absorb the target analytical compounds from the water samples.

Ground-water samples were discharged directly into the filtering device through tubing connected to sample taps at water-supply wells or the discharge outlet on sampling pumps. 


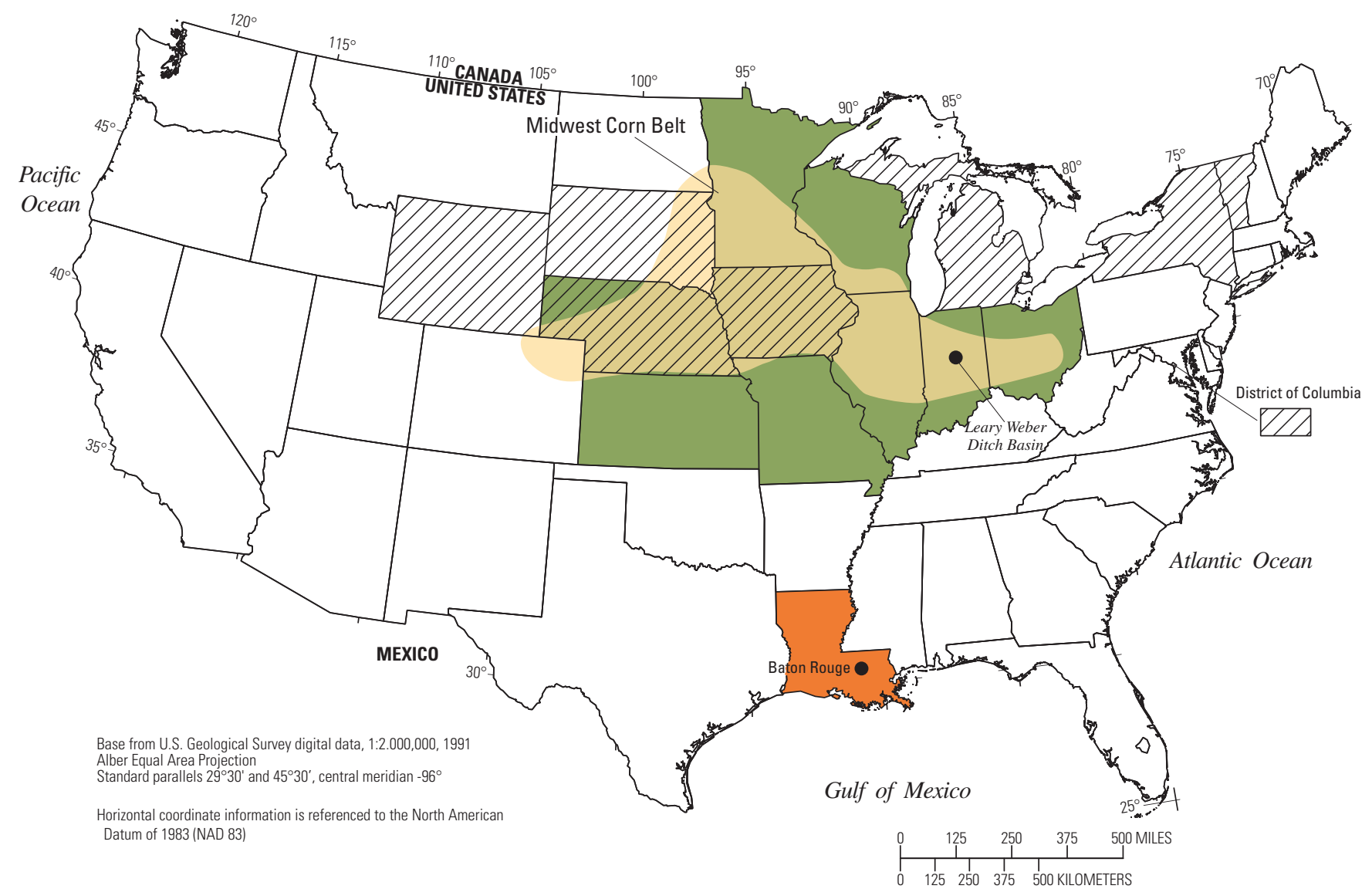

EXPLANATION

Nine Midwestern States

Mississippi River glyphosate study

Sensitive environments study

Figure 5. Location of Midwest Corn Belt and Toxic Substances Hydrology Program studies where samples were collected for analysis of glyphosate, its degradation product, aminomethylphosphonic acid, and glufosinate, 2001-06.

The water samples from each surface-water site were composited in a single container and filtered through a nominal $0.7-\mu \mathrm{m}$ glass-fiber filter using a peristaltic pump. Filters were preconditioned with about $200 \mathrm{~mL}$ of sample water prior to collection of the sample. The filtrate for analysis was collected in baked 125-mL amber glass bottles with Teflon-lined lids. All samples were chilled immediately and shipped to OGRL and stored at approximately $4^{\circ} \mathrm{C}$ until analyzed.

Rainfall samples were collected by a refrigerated wetdeposition sampler as described in Baker and others (2006). Soil samples were collected at three depths at each site (for a total of nine samples per site for every sample-collection period). A soil core sampler was used to collect samples from 0-6 in., 6-12 in., and 12-18 in. at each of the three randomly chosen sample locations. Clean aluminum sampling tubes were inserted in the core sampler and the first 6-in. section (0-6 in.) of soil was extracted. The section was removed from the sampling tube (with nitrile gloves) and placed in a labeled glass jar. Clean sampling tubes were inserted in the core sampler, and the second 6-in. section (6-12 in.) of soil was extracted from the same hole. The same procedure was used on the third 6-in. section (12-18 in.) of soil.

\section{Analytical Methods and Procedures}

\section{Liquid Chromatography/Mass Spectrometry and Liquid Chromatography/Tandem Mass Spectrometry}

The analytical method for glyphosate, AMPA, and glufosinate compounds in water is described in Lee and others $(2002 a, b)$ and is suitable for the determination of concentrations, using online SPE and LC/MS (from 2001 to 2004), and was modified to use an LC/MS/MS method in 2004. 


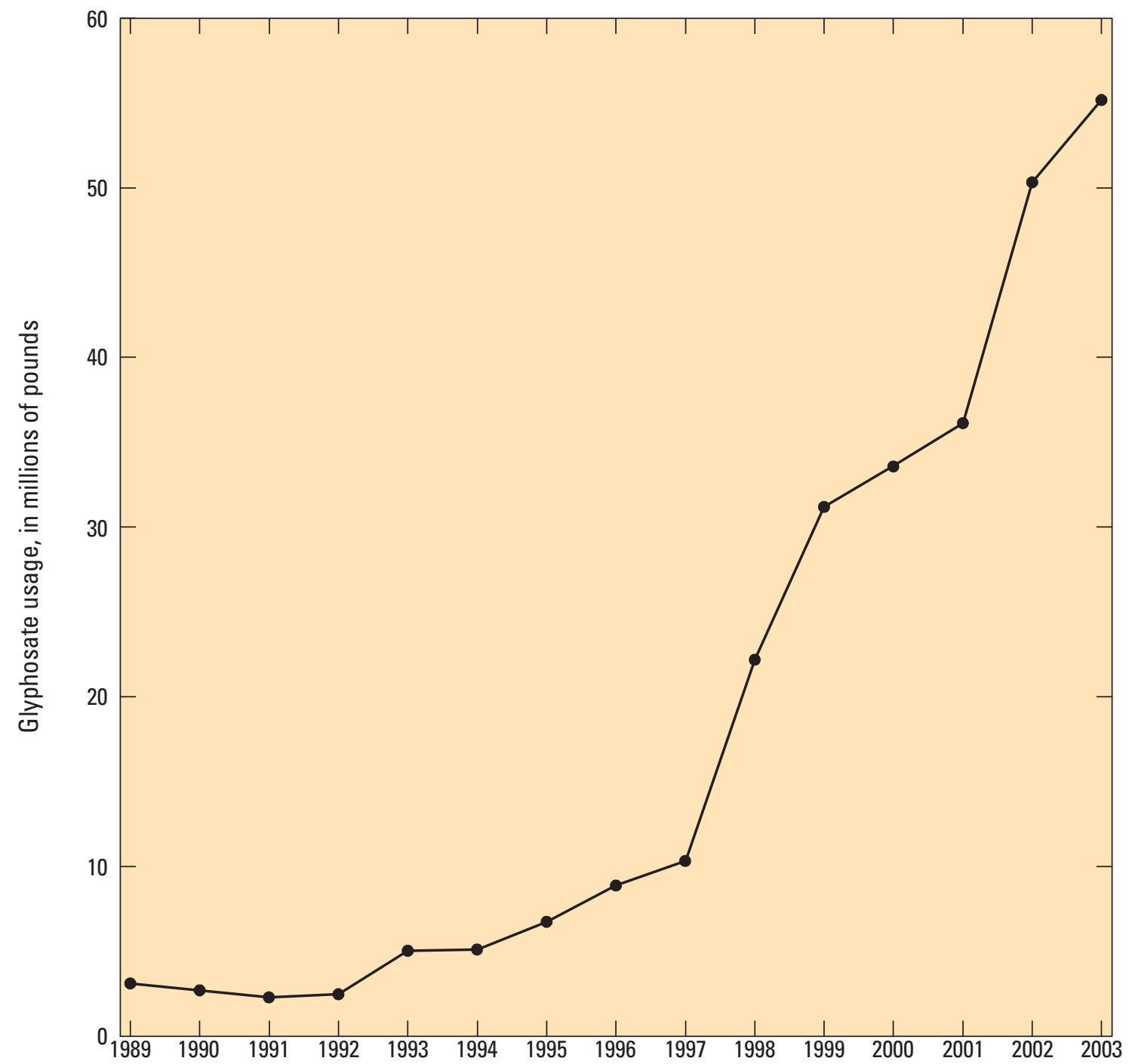

Figure 6. Glyphosate use in nine Midwestern States, 1989-2003 (data from U.S. Department of Agriculture, 2006).

\section{Materials}

Analytical grade standards of glyphosate, AMPA, and glufosinate were obtained from ChemService, Inc. (West Chester, Pennsylvania) for use as internal standards. Stable isotope-labeled glyphosate $\left(2-{ }^{13} \mathrm{C},{ }^{15} \mathrm{~N}\right)$ purchased from Cambridge Isotope Laboratories (Andover, Massachusetts) was used as an internal standard for LC/MS method as described in Lee and others (2002a). Stable isotope-labeled $\left[{ }^{13} \mathrm{C}_{2},{ }^{15} \mathrm{~N}\right.$ - glyphosate (glyphosate +3 ) $]$ and $\left[{ }^{13} \mathrm{C},{ }^{15} \mathrm{~N}\right.$, methylene- $\mathrm{D}_{2}$ - AMPA (AMPA +4)] were purchased from $\mathrm{GmbH}$ Company (Germany). The second isotope-labeled AMPA $\left[{ }^{13} \mathrm{C}\right.$, ${ }^{15} \mathrm{~N}$ - AMPA (AMPA +2)] came from Cerfilliant Corporation (Round Rock, Texas). There was no stabilized isotopelabeled internal standard available for glufosinate. The internal standard cysteic acid was obtained from Sigma, St. Louis, Missouri. All solvents were high-performance liquid chromatography (HPLC) grade.

\section{Derivatization of Samples}

\section{Water}

As described in Lee and others (2002a), each water sample (ground water, surface water, rainfall) was derivatized by dispensing $10 \mathrm{~mL}$ into two labeled, $19-\mathrm{mL}$, screwcapped plastic tubes. The sample in the tube labeled "standard addition" was fortified with $1 \mu \mathrm{g} / \mathrm{L}$ of each compound to be analyzed. Internal standard solutions were added to both tubes, the sample was buffered to $\mathrm{pH} 9.0$ standard units by adding borate buffer, and after mixing, a solution of 9-fluorenylmethylchloroformate (FMOC) was added to all tubes. Derivatization was conducted in the dark in a water bath at $40^{\circ} \mathrm{C}$. After 24 hours, the reaction was stopped and stabilized by adding 2-percent phosphoric acid, then tubes were stored in a refrigerator at $4^{\circ} \mathrm{C}$ until analyzed. 
Soil

Soil samples were placed on an aluminum foil sheet, and a spatula was used to break up and mix the sample. Five grams $(5 \mathrm{~g})$ of soil sample were weighed into a labeled $50-\mathrm{mL}$ plastic centrifuge tube using at least ten 0.5 -g aliquots from different areas of the sample. Twenty-five milliliters $(25 \mathrm{~mL})$ of $0.5 \mathrm{M}$ potassium hydroxide $(\mathrm{KOH})$ were added to the sample tube, which was placed on a mechanical shaker for $45 \mathrm{~min}$ at medium speed, then centrifuged for $10 \mathrm{~min}$ to extract the compounds.

Five milliliters $(5 \mathrm{~mL})$ of the centrifuged $\mathrm{KOH}$ soil extracts were pipetted into a new labeled $50-\mathrm{mL}$ centrifuged tube to which $5.1 \mathrm{~mL}$ of $0.5 \mathrm{M}$ hydrochloric acid $(\mathrm{HCl})$ were added. Five-tenths $\mathrm{M} \mathrm{HCl}$ was added drop-wise until a $\mathrm{pH}$ of 3.0 to 4.0 standard units was obtained. Ten milliliters $(10 \mathrm{~mL})$ of borate buffer were added by drop until $\mathrm{pH}$ was adjusted to 9.0 standard units. The internal standard and FMOC solutions then were added to the sample eluate and placed in a $40^{\circ} \mathrm{C}$ water bath for 24 hours. The reaction was stopped and stabilized by adding 2-percent phosphoric acid, then tubes were stored in a refrigerator at $4^{\circ} \mathrm{C}$ until analyzed.

\section{Online Solid-Phase Extraction}

A Prospekt automated online SPE instrument with the Triathlon autosampler (Spark-Holland, The Netherlands) were used to extract the samples. Each sample and the matching standard-addition sample were loaded into the sample tray of the autosampler. The SPE instrument were loaded with cartridges. The Prospekt had one cartridge clamp to rinse the cartridge and load the sample and another cartridge clamp to elute the previously prepared cartridge. The sample lines were rinsed and deactivated with a sodium hydroxide $(\mathrm{NaOH})$ and ethylenediaminetetraacetic acid (EDTA) solution between samples. The SPE cartridge was rinsed with methanol and conditioned with reagent water, and then $10 \mathrm{~mL}$ of soil sample were loaded onto the cartridge from the autosampler at a rate of $2 \mathrm{~mL} / \mathrm{min}$. The cartridge was washed with reagent water at the same rate for $15 \mathrm{sec}$. The SPE cartridge then was eluted with $750 \mu \mathrm{L}$ of acetonitrile (ACN) and by diverting the liquid chromatography (LC) mobile-phase stream through the cartridge. The mobile-phase composition was set to elute the compounds of interest and leave the excess derivatization reagent on the cartridge (Lee and others, 2002a).

\section{Liquid Chromatography/Mass Spectrometry}

Samples were analyzed on a Hewlett Packard (Wilmington, Delaware) model 1100 high-performance liquid chromatograph (HPLC) with autoinjector and mass spectrometry detector. The concentration of each compound was calculated by determining the ratio of the compound to the internal standard to the ratio of the same compound in the standardaddition sample minus the ratio of the sample. The sample and standard-addition sample were analyzed sequentially using the same method and instruments (Lee and others, 2002a). The reporting limit for the LC/MS method was $0.10 \mu \mathrm{g} / \mathrm{L}$ from 2001 through 2004.

\section{Liquid Chromatography/Tandem Mass Spectrometry}

Samples were analyzed on an Agilent 1100 series liquid chromatography (LC) system (Wilmington, Delaware) and a Waters Quattro Micro atmospheric pressure ionization (API) tandem mass spectrometer (MS/MS) system with electrospray ionization in negative-ion mode (Milford, Massachusetts). A Luna C-18 (2) analytical column (Phenomenex, Torrance, California) was used to separate glyphosate, AMPA, and glufosinate. The LC column was equilibrated with the mobile phase approximately 2 hours prior to analysis. A reporting level of $0.02 \mu \mathrm{g} / \mathrm{L}$ for the LC/MS/MS method has been used since 2004.

\section{Quality Assurance}

\section{Sample Collection}

All water samples for these studies were collected either by or under the direction of USGS personnel in accordance with a written work plan for the study. Field blank and equipment blank samples were collected to ensure cross contamination did not occur due to contaminated equipment. Field replicate samples were collected to document analytical variability. Quality-control samples included 28 field blanks and 184 field replicates. The blanks were free of glyphosate, AMPA, and glufosinate. Of the 184 field replicates, 135 samples contained analytes, whereas 49 samples were nondetections. All replicates matched the regular sample within 20 percent. The analytical results from these samples are listed along with the results from the regular samples in tables 3 through 6 at the end of this report.

\section{Internal Standards}

Internal standards were added to correct quantitative results for slight differences in extract volume as well as to compensate for differences in the injected sample volume. Internal standards also were used to monitor instrument conditions, such as extract injection errors, retention time shifts, or instrument abnormalities or malfunctions.

\section{Laboratory Blank}

The laboratory blank was reagent water prepared to monitor the entire sample preparation and analytical procedure for 
possible laboratory contamination. The blank was considered acceptable when a compound was undetected or detected at less than the minimum reporting level (MRL; Timme, 1995). On the basis of data analysis, there were no interferences in the laboratory blanks.

\section{Laboratory Spike}

The percentage recovery from the laboratory reagent spike was calculated from the spiking procedure described. The recovery was used to monitor the method performance for the sample preparation without considering the effects of sample matrix. The laboratory spike results were compiled for long-term recovery performance used for creating control limits and charts. If the recovery of a compound was not within control limits, the source of the problem was identified and corrected before continuing the procedure.

\section{Instrumental Analysis Quality Control}

A sample analytical sequence used for this method is listed in table 1. Sample extracts were analyzed in an instrument sequence to provide additional information to facilitate corrective actions that might be required if performance criteria were not met.

\section{Concentrations of Glyphosate, Aminomethylphosphonic Acid, and Glufosinate}

A summary of ground-water, surface-water, rainfall, and soil samples collected for State cooperative studies, the National Stream Quality Accounting Network Program, the National Water-Quality Assessment Program, and the Toxic Substances Hydrology Program, and analyzed by OGRL for glyphosate, AMPA, and glufosinate is presented in table 2.

Analytical results for glyphosate, AMPA, and glufosinate in 2,135 ground- and surface-water samples, 14 rainfall samples, and 193 soil samples collected from various study areas throughout the United States are provided in tables 3 through 6 at the end of this report. Laboratory duplicates also are shown in tables 3 through 6 but not included in the sample count in table 2 .

\section{State Cooperative Studies}

During the State cooperative studies, 271 ground- and 281 surface-water samples were analyzed (table 3 at end of report) from 10 States ranging from Washington to Vermont and south to Mississippi. In the ground-water samples analyzed, there was no glyphosate detection and only one AMPA detection of $0.33 \mu \mathrm{g} / \mathrm{L}$ in water from a well in Kansas. There also were no samples from the State cooperative studies with a reportable glufosinate concentration. The lack of groundwater detections may have been the result of a higher reporting level $(0.1 \mu \mathrm{g} / \mathrm{L})$ prior to 2004 compared to a reporting level $(0.02 \mu \mathrm{g} / \mathrm{L})$ that was later achieved with the LC/MS/MS system. Glyphosate was detected in about 50 percent of the surface-water samples. A maximum glyphosate concentration of $99 \mu \mathrm{g} / \mathrm{L}$ was found in a sample collected in April 2002 from a site in Mississippi surrounded by cotton fields being treated with glyphosate to prepare for planting.

\section{National Stream Quality Accounting Network (NASQAN) Program}

Twenty-seven samples (table 4 at end of report) were collected from four NASQAN river sites in the Mississippi River Basin during 2002 (fig. 2). Overall, AMPA was detected at greater frequencies than glyphosate in these surface-water samples. There were two glyphosate detections with concentrations of 0.33 and $0.14 \mu \mathrm{g} / \mathrm{L}$, and 17 AMPA detections with a maximum concentration of $0.38 \mu \mathrm{g} / \mathrm{L}$ (tables 2 and 4 ).

\section{National Water-Quality Assessment (NAW0A) Program}

A total of 1,093 water samples were collected from 23 study units for the NAWQA Program during 2001-06 (fig. 3 and table 5 at end of report). In 485 ground-water samples, maximum concentrations were $0.67 \mu \mathrm{g} / \mathrm{L}$ for glyphosate (28 detections) and $0.62 \mu \mathrm{g} / \mathrm{L}$ for AMPA (47 detections). In 608 surface-water samples, maximum concentrations were $9.7 \mu \mathrm{g} / \mathrm{L}$ for glyphosate (196 detections) and $8.7 \mu \mathrm{g} / \mathrm{L}$ for AMPA (313 detections). There were two detections (0.11 and $0.56 \mu \mathrm{g} / \mathrm{L}$ ) of glufosinate in surface water from the Mississippi Embayment and White River Basin NAWQA study units. These results concur with results from previous studies that found the glyphosate degradation product, AMPA, occurs more frequently or at similar or higher concentrations than glyphosate, whereas glufosinate was seldom found in the environment (Scribner and others, 2003; Battaglin and others, 2005).

\section{Toxic Substances Hydrology (Toxics) Program}

The USGS Toxic Substances Hydrology Program funded several studies focused on the fate and transport of glyphosate and AMPA in ground and surface water including overland flow, tile-drain flow, rainfall, and soil since 2001 (table 6 at end of report). Overall, these studies show that glyphosate and AMPA were detected more frequently in surface water than in ground water, and AMPA was detected at greater frequencies than glyphosate in surface water. The data also indicate that levels of glyphosate and AMPA may persist in the soil from year to year (Baker and others, 2006). Table 2 shows 
Table 1. Example of analytical sequence for use in determining glyphosate, aminomethylphosphonic acid, and glufosinate in water samples.

[Study code, code used by study to identify samples; LCGY 102, glyphosate method acronym and run number; COB, carryover blank sample; ppb, parts per billion; L, laboratory duplicate; A, B, C, letters assigned to first, second, third sample bottles, respectively, at login; --, not applicable]

\begin{tabular}{|c|c|c|c|c|}
\hline \multicolumn{2}{|c|}{ LCGY run sheet } & \multirow{3}{*}{ LCGY 102} & \multirow{3}{*}{$\begin{array}{c}\text { Date: 08/24/05 } \\
\text { Derivatized }\end{array}$} & \multirow{3}{*}{ Login iniitials } \\
\hline \multicolumn{2}{|c|}{ Method } & & & \\
\hline Vial number & Study code & & & \\
\hline 1,2 & -- & COB A \& $1.0 \mathrm{ppb}$ & $08 / 25 / 05$ & EG \\
\hline 3,4 & LIG & $05254 \mathrm{~A}$ & $08 / 25 / 05$ & EG \\
\hline 5,6 & PUB & $05255 \mathrm{~A}$ & $08 / 25 / 05$ & EG \\
\hline 7,8 & $\mathrm{RCM}$ & $05263 \mathrm{~A}$ & 08/25/05 & EG \\
\hline 9,10 & LIG & $05264 \mathrm{~A}$ & $08 / 25 / 05$ & EG \\
\hline 11,12 & LIG & $05265 \mathrm{~A}$ & 08/25/05 & EG \\
\hline 13,14 & LIG & $05266 \mathrm{~A}$ & $08 / 25 / 05$ & EG \\
\hline 15,16 & LIG & $05275 \mathrm{~A}$ & 09/01/05 & EG \\
\hline 17,18 & LIG & $05276 \mathrm{~A}$ & 09/01/05 & EG \\
\hline 19,20 & LIG & $05277 \mathrm{~A}$ & 09/01/05 & EG \\
\hline 21,22 & PUG & $05312 \mathrm{~A}$ & 09/01/05 & EG \\
\hline 23,24 & LIG & $05254 \mathrm{~L}$ & 08/25/05 & EG \\
\hline 25,26 & -- & COB B \& $2.0 \mathrm{ppb}$ & $08 / 25 / 05$ & EG \\
\hline 27,28 & PUG & $05313 \mathrm{~A}$ & 09/01/05 & EG \\
\hline 29,30 & LIG & $05318 \mathrm{~A}$ & 09/01/05 & EG \\
\hline 31,32 & LIG & $05319 \mathrm{~A}$ & 09/01/05 & EG \\
\hline 33,34 & LIG & $05324 \mathrm{~A}$ & 09/07/05 & EG \\
\hline 35,36 & LIG & $05325 \mathrm{~A}$ & 09/07/05 & EG \\
\hline 37,38 & LIG & $05326 \mathrm{~A}$ & 09/07/05 & EG \\
\hline 39,40 & USG & $05335 \mathrm{~A}$ & 09/07/05 & EG \\
\hline 41,42 & LIG & 05349 A & 09/07/05 & EG \\
\hline 43,44 & $\mathrm{RCM}$ & $05350 \mathrm{~A}$ & 09/07/05 & EG \\
\hline 45,46 & PUB & $05351 \mathrm{~A}$ & 09/07/05 & EG \\
\hline 47,48 & SKF & $05353 \mathrm{~A}$ & 09/13/05 & EG \\
\hline 49,50 & SKF & $05354 \mathrm{~A}$ & 09/13/05 & EG \\
\hline 51,52 & SKF & $05355 \mathrm{~A}$ & 09/13/05 & EG \\
\hline 53,54 & SKF & $05356 \mathrm{~A}$ & 09/13/05 & EG \\
\hline 55,56 & SKF & $05357 \mathrm{~A}$ & 09/13/05 & EG \\
\hline 57,58 & ILG & $05367 \mathrm{~A}$ & 09/13/05 & EG \\
\hline 59,60 & PUB & $05313 \mathrm{~L}$ & 09/01/05 & EG \\
\hline 61,62 & -- & COB C \& $1.0 \mathrm{ppb}$ & 09/01/05 & EG \\
\hline
\end{tabular}


Table 2. Summary of glyphosate, aminomethylphosphonic acid, and glufosinate concentrations analyzed by the U.S. Geological Survey Organic Geochemistry Research Laboratory, Lawrence, Kansas, for ground-water, surface-water, rainfall, and soil samples collected for State cooperative, National Stream Quality Accounting Program, National Water-Quality Assessment Program, and Toxic Substances Hydrology Program studies, 2001-06.

$[--$, no data; $<$, less than; $\mu \mathrm{g} / \mathrm{kg}$, microgram per kilogram; $\mu \mathrm{g} / \mathrm{L}$, microgram per liter $]$

\begin{tabular}{|c|c|c|c|c|c|c|c|c|c|c|}
\hline \multirow[b]{2}{*}{ U.S. Geological Survey studies } & \multirow[b]{2}{*}{$\begin{array}{l}\text { Num- } \\
\text { ber of } \\
\text { sam- } \\
\text { ples }\end{array}$} & \multicolumn{3}{|c|}{ Glyphosate } & \multicolumn{3}{|c|}{$\begin{array}{l}\text { Aminomethylphosphonic } \\
\text { acid }\end{array}$} & \multicolumn{3}{|c|}{ Glufosinate } \\
\hline & & $\begin{array}{l}\text { Num- } \\
\text { ber of } \\
\text { detec- } \\
\text { tions }\end{array}$ & $\begin{array}{l}\text { Maxi- } \\
\text { mum } \\
\text { con- } \\
\text { centra- } \\
\text { tion }\end{array}$ & $\begin{array}{l}\text { Mini- } \\
\text { mum } \\
\text { con- } \\
\text { centra- } \\
\text { tion }\end{array}$ & $\begin{array}{c}\text { Num- } \\
\text { ber of } \\
\text { detec- } \\
\text { tions }\end{array}$ & $\begin{array}{l}\text { Maxi- } \\
\text { mum } \\
\text { con- } \\
\text { centra- } \\
\text { tion }\end{array}$ & $\begin{array}{c}\text { Mini- } \\
\text { mum } \\
\text { con- } \\
\text { cen- } \\
\text { tra- } \\
\text { tion }\end{array}$ & $\begin{array}{c}\text { Num- } \\
\text { ber of } \\
\text { detec- } \\
\text { tions }\end{array}$ & $\begin{array}{l}\text { Maxi- } \\
\text { mum } \\
\text { con- } \\
\text { centra- } \\
\text { tion }\end{array}$ & $\begin{array}{l}\text { Mini- } \\
\text { mum } \\
\text { con- } \\
\text { cen- } \\
\text { tration }\end{array}$ \\
\hline \multicolumn{11}{|c|}{ Ground water $(\mu \mathrm{g} / \mathrm{L})$} \\
\hline State cooperative studies & 271 & 0 & $<0.10$ & $<0.10$ & 1 & 0.33 & $<0.10$ & 0 & $<0.10$ & $<0.10$ \\
\hline $\begin{array}{l}\text { National Stream Quality Accounting } \\
\text { Network Program (NASQAN) }\end{array}$ & -- & -- & -- & -- & -- & -- & -- & -- & -- & -- \\
\hline $\begin{array}{l}\text { National Water-Quality Assessment } \\
\text { Program (NAWQA) }\end{array}$ & 485 & 28 & .67 & .02 & 47 & .62 & .02 & 0 & $<.10$ & $<.10$ \\
\hline \multicolumn{11}{|l|}{$\begin{array}{l}\text { Toxic Substances Hydrology } \\
\text { Program (Toxics) }\end{array}$} \\
\hline Reconnaissance Data & -- & -- & -- & -- & -- & -- & -- & -- & -- & -- \\
\hline Lower Mississippi River Basin & -- & -- & -- & -- & -- & -- & -- & -- & -- & -- \\
\hline Sensitive Environments & -- & -- & -- & -- & -- & -- & -- & -- & -- & -- \\
\hline Leary Weber Ditch Basin & 117 & 40 & 4.7 & .02 & 85 & 2.6 & .03 & 0 & $<.02$ & $<.02$ \\
\hline \multicolumn{11}{|c|}{ Surface water $(\mu \mathrm{g} / \mathrm{L})$} \\
\hline State cooperative studies & 281 & 143 & 99 & .02 & 165 & 22 & .04 & 0 & $<.02$ & $<.02$ \\
\hline $\begin{array}{l}\text { National Stream Quality Accounting } \\
\text { Network Program (NASQAN) }\end{array}$ & 27 & 2 & .33 & .14 & 17 & .38 & .12 & 0 & $<.10$ & $<.10$ \\
\hline $\begin{array}{l}\text { National Water-Quality Assessment } \\
\text { Program (NAWQA) }\end{array}$ & 608 & 196 & 9.7 & .03 & 313 & 8.7 & .03 & 2 & .56 & .11 \\
\hline \multicolumn{11}{|l|}{$\begin{array}{l}\text { Toxic Substances Hydrology } \\
\text { Program (Toxics) }\end{array}$} \\
\hline Reconnaissance Data & 171 & 63 & 8.7 & .11 & 117 & 3.6 & .10 & 2 & .26 & .14 \\
\hline Lower Mississippi River Basin & 35 & 0 & $<.10$ & $<.10$ & 31 & .38 & .10 & 0 & $<.10$ & $<.10$ \\
\hline Sensitive Environments & 76 & 31 & 328 & .02 & 30 & 41 & .02 & 1 & .05 & $<.02$ \\
\hline Leary Weber Ditch Basin & 64 & 54 & 427 & .03 & 52 & 29 & .06 & 2 & 1.5 & .24 \\
\hline
\end{tabular}

Toxic Substances Hydrology

Program (Toxics)

\begin{tabular}{cccccccccccc} 
Leary Weber Ditch Basin & 14 & 12 & 1.1 & .03 & 12 & .47 & .02 & 0 & $<.02$ & $<.02$ \\
\hline \multicolumn{10}{c}{ Soil $(\mu \mathrm{g} / \mathrm{kg})$} \\
\hline
\end{tabular}

Toxic Substances Hydrology

Program (Toxics)

Leary Weber Ditch Basin

$193 \quad 119 \quad 476$

1.0

154

956

1.0

1

$1.0<1.0$ 
the number of ground- and surface-water, rainfall, and soil samples collected, number of detections, and the minimum and maximum concentrations.

\section{Reconnaissance Data From 51 Streams in Nine Midwestern States}

During 2002, 171 samples were collected from 51 streams in nine Midwestern States during three periods of runoff (table 6 at end of report). Glyphosate was detected in 63 samples with a maximum concentration of $8.7 \mu \mathrm{g} / \mathrm{L}$, AMPA was detected in 117 samples with a maximum concentration of $3.6 \mu \mathrm{g} / \mathrm{L}$, and glufosinate was detected in two samples at concentrations of 0.26 and $0.14 \mu \mathrm{g} / \mathrm{L}$ (table 2; Scribner and others, 2003; Battaglin and others, 2005).

\section{Lower Mississippi River Basin}

Results from systematic water-quality sampling from sites in the lower Mississippi River were used to investigate the occurrence, fate, and transport of selected pesticides and their degradation products (U.S. Geological Survey, 2006a). Water samples were collected from the lower Mississippi River at Baton Rouge, Louisiana (site 07374000) (fig. 5) during April 1991 through December 2003 to help determine the occurrence and transport of selected herbicides, their degradation products, and nutrients in the lower Mississippi River Basin and the delivery to the Gulf of Mexico (Scribner and others, 2006).

During 2001-03, water samples were collected twice monthly to study the occurrence of glyphosate, AMPA, and glufosinate. There were no detections of either glyphosate or glufosinate in the 35 samples; however, there were 31 detections of AMPA, with a maximum concentration of $0.38 \mu \mathrm{g} / \mathrm{L}$ (tables 2 and 6).

\section{Sensitive Environments Study}

Vernal pools are sensitive environments of natural water that provide critical habitats for many species, including amphibians. These small water bodies are not always protected by pesticide label requirements for no-spray buffer zones, and the occurrence of pesticides detected is poorly documented. In the Sensitive Environments study (W.A. Battaglin, USGS written commun., June 19, 2007), glyphosate was detected in 31 of 76 samples with a maximum concentration of $328 \mu \mathrm{g} / \mathrm{L}$, whereas AMPA was detected in 30 samples with a maximum concentration of $41 \mu \mathrm{g} / \mathrm{L}$. Glufosinate was detected in one sample at $0.05 \mu \mathrm{g} / \mathrm{L}$ (table 2).

\section{Leary Weber Ditch Basin, Indiana}

A joint collaboration between Toxics and NAWQA was formed to study the agricultural chemicals source, transport, and fate within the Leary Weber Ditch Basin, a part of the White River Basin, a NAWQA study unit in Indiana (figs. 4 and 5). In 2004-05, samples were collected from various hydrologic compartments in the Leary Weber Ditch Basin and analyzed for glyphosate, AMPA, and glufosinate.

Agricultural chemicals were found in Leary Weber Ditch and every other hydrologic compartment sampled during 2004-05 (Baker and others, 2006). Of the 117 ground-water samples analyzed, glyphosate was detected in 40 samples with a maximum of $4.7 \mu \mathrm{g} / \mathrm{L}$, and AMPA was detected in $85 \mathrm{sam}-$ ples with a maximum of $2.6 \mu \mathrm{g} / \mathrm{L}$ (tables 2 and 6). There were no glufosinate detections. Glyphosate was detected in 54 of the 64 surface-water samples with a maximum concentration of $427 \mu \mathrm{g} / \mathrm{L}$, and AMPA was detected in 52 of the 64 samples with a maximum concentration of $29 \mu \mathrm{g} / \mathrm{L}$ at Mohawk, Indiana (table 6). In the Leary Weber Ditch, 51 of 64 water samples analyzed contained both glyphosate and AMPA. Higher concentrations in the Leary Weber Ditch occurred soon after application to fields and coincided with rainfall. Glufosinate was found in two surface-water samples from the Leary Weber Ditch at concentrations of 1.5 and $0.24 \mu \mathrm{g} / \mathrm{L}$ (tables 2 and 6).

Both glyphosate and AMPA were detected in 12 of the 14 rainfall samples collected. Concentrations ranged from 0.02 to $1.1 \mu \mathrm{g} / \mathrm{L}$. Glufosinate was not detected in any of the rainfall samples.

To examine the potential transport of glyphosate and AMPA through the unsaturated zone, soil cores were collected from April through October 2004 from both the north and south field sites in the Leary Weber Ditch Basin. Agricultural practices were the same for both fields. The maximum concentration for glyphosate was $476 \mu \mathrm{g} / \mathrm{kg}$, whereas the maximum concentration for AMPA was $956 \mu \mathrm{g} / \mathrm{kg}$ (table 2). The data show that the maximum concentration of AMPA during preapplication (April) was $23 \mu \mathrm{g} / \mathrm{kg}$. These data results may indicate that trace levels of glyphosate can persist in the soil from year to year (Baker and others, 2006). One north field soil sample (6-12 in. depth) contained glufosinate at a concentration of $1.0 \mu \mathrm{g} / \mathrm{kg}$ (table 6).

\section{Summary}

The Organic Geochemistry Research Laboratory (OGRL) was established by the USGS in Lawrence, Kansas, to identify contaminants that may have deleterious effects on water quality or be useful indicators of geochemical transport processes and to conduct research on the fate and transport of contaminants in the hydrogeologic system. Essential analytical methods have been developed using state-of-the-art liquid chromatography and mass spectrometry to accomplish this.

This report presents results of analyses by OGRL for glyphosate, its degradation product, aminomethylphosphonic acid (AMPA), and glufosinate in 2,135 ground- and surfacewater samples, 14 rainfall samples, and 193 soil samples collected from 2001 through 2006. The data are used to document 
the occurrence, distribution, and concentrations of glyphosate, its degradation product, aminophosphonic acid (AMPA), and glufosinate at locations across the United States. The data were collected as part of various ongoing USGS studies, such as those done for Federal and State cooperative agencies, the National Stream Quality Accounting Network (NASQAN) Program, the National Water-Quality Assessment (NAWQA) Program, and the Toxic Substances Hydrology (Toxics) Program.

Glyphosate is among the most commonly used herbicides in the world. Its use has substantially increased in the United States with the introduction of genetically modified glyphosate-resistant corn and soybeans; however, it also is used extensively in yards, gardens, parks, and other nonagricultural areas. Studies show glyphosate is degraded primarily by microbial processes producing aminomethylphosphonic acid (AMPA). Samples also were analyzed for glufosinate, a broad-spectrum contact herbicide, used to control a wide range of weeds after the crop emerges or for total vegetation control on land not used for cultivation.

Results show that AMPA was detected more frequently and occurred at similar or higher concentrations than the parent compound, glyphosate, whereas glufosinate was seldom found in the environment. Further study also shows that glyphosate and AMPA were detected more frequently in surface water than in ground water. Both glyphosate and AMPA were detected in rainfall samples in Indiana. The data also indicate that trace levels of glyphosate and AMPA may persist in the soil from year to year. These data were valuable for acquiring information about the occurrence, fate, and transport of these compounds and to show the importance of analyzing not only glyphosate but also AMPA in water-quality studies.

Analytical results show that the methods developed at OGRL were valuable for acquiring information about the fate and transport of glyphosate, AMPA, and glufosinate in ground and surface water, rainfall, and soil. The data also indicate the importance of including both parent compounds and their degradation products in herbicide studies.

\section{References Cited}

Baker, N.T., Stone, W.W., Wilson, J.T., and Meyer, M.T., 2006, Occurrence and transport of agricultural chemicals in Leary Weber Ditch Basin, Hancock County, Indiana, 2003-04: U.S. Geological Survey Scientific Investigations Report 2006-5251, 44 p.

Battaglin, W.A., and Goolsby, D.A., 1999, Are shifts in herbicide use reflected in concentration changes in Midwestern rivers?: Environmental Science \& Technology, v. 33, p. 2917-2925.
Battaglin, W.A., Kolpin, D.W., Scribner, E.A., Kuivila, K.M., and Sandstrom, M.W., 2005, Glyphosate, other herbicides, and transformation products in Midwestern streams, 2002: Journal of American Water Resources Association, v. 41, no. 2, p. 323-332.

Baylis, A.D., 2000. Why glyphosate is a global herbicidestrengths, weaknesses and prospects: Pesticide Management Science, v. 56, no. 4, p. 299-308.

Buxton, H.T., 2000, USGS Toxic Substances Hydrology Program, 2000: U.S. Geological Survey Fact Sheet 062-00, 4 p.

Carlisle, S.M., and Trevors, J.T., 1988, Glyphosate in the environment: Water, Air, and Soil Pollution, v. 39, p. 409-420.

Coupe, R.H., Jr., and Goolsby, D.A., 1999, Monitoring the water quality of the Nation's large rivers-Mississippi River Basin NASQAN Program: U.S. Geological Survey Fact Sheet 055-99, 6 p.

Cox, Caroline, 1996, Herbicide fact sheet—glufosinate: Journal of Pesticide Reform, Winter 1996, v. 16, no. 4, p. 15-19.

Cox, Caroline, 2004, Glyphosate herbicide fact sheet: Journal of Pesticide Reform, Winter 2004, v. 24, no. 4, p. 10-15.

Extension Toxicology Network, 1994, Glyphosate: Information available on the Web, accessed April 19, 2006, at http://pmep.cce.cornell.edu/profiles/extoxnet/dienochlorglyphosate/glyphosate-ext.htmll

Friends of the Earth, 2001, Health and environmental impacts on glufosinate ammonium: Information available on the Web, accessed February 7, 2007, at http:/www.foe.co.uk/ resource/reports/impacts_glufosinate_ammon.pdf

Gilliom, R.J., Barbash, J.E., Crawford, C.G., Hamilton, P.A., Martin, J.D., Nakagaki, Naomi, Nowell, L.H., Scott, J.C., Stackelberg, P.E., Thelin, G.P., and Wolock, D.M., 2006, Pesticides in the Nation's streams and ground water, 1992-2001: U.S. Geological Survey Circular 1291, 172 p.

Gilliom, R.J., and Hamilton, P.A., 2006, Pesticides in the Nation's streams and ground water, 1992-2001 - a summary: U.S. Geological Survey Fact Sheet 2006-3028, 6 p.

Hooper, R.P., Aulenbach, B.T., and Kelly, V.J., 2001, The National Stream Quality Accounting Network-a fluxbased approach to monitoring the water quality of large rivers: Hydrological Processes, v. 15, p. 1251-1269.

Hooper, R.P., Goolsby, D.A., Rickert, D.A., and McKenzie, S.W., 1997, A river-basin perspective on monitoring water quality: U.S. Geological Survey Fact Sheet 055-97, 6 p. 
Iowa State University, 1997, Glyphosate: Iowa State University Agronomy, information available on the Web, accessed September 23, 2005, at http:/www.agron.iastate. edu/ weeds/Ag317/manage/herbicide/glyphosate.htm

Kiely, Thomas, Donaldson, David, and Grube, Arthur, 2004, Pesticides industry sales and usage-2000 and 2001 market estimates: U.S. Environmental Protection Agency, Office of Prevention, Pesticides, and Toxic Substances, Office of Pesticide Programs, information available on the Web, accessed April 20, 2006, at http://www.epa.gov/oppbeadl/ pestsales/01pestsales/market_estimates2001.pdf

Kolpin, D.W., Thurman, E.M., Lee, E.A., Meyer, M.T., Furlong, E.T., and Glassmeyer, S.T., 2006, Urban contributions of glyphosate and its degradate AMPA to streams in the United States: Science of Total Environment, v. 354, p. 191-197.

Lee, E.A., Strahan, A.P., and Thurman, E.M., 2002a, Methods of analysis by the U.S. Geological Survey Organic Geochemistry Research Group-determination of glyphosate, aminomethylphosphonic acid, and glufosinate in water using online solid-phase extraction and high-performance liquid chromatography/mass spectrometry: U.S. Geological Survey Open-File Report 01-454, 13 p.

Lee, E.A., Zimmerman, L.R., Bhullar, B.S., and Thurman, E.M., 2002b, Linker-assisted immunoassay and liquid chromatography/mass spectrometry for the analysis of glyphosate: Analytical Chemistry, v. 74, p. 4937-4943.

Monsanto Company, 2002, Backgrounder-history of Monsanto's glyphosate herbicides: Information available on the Web, accessed November 3, 2006, at http://www.monsanto. com/monsanto/content/products/productivity/roundupd Response_ISIS_apr_06.pdff

Pesticide Action Network UK, 1998, Glufosinate ammonium fact sheet: Information available on the Web, accessed September 23, 2006, at http://www.pan_uk.org/pestnews actives/glufosin.htm

Rueppel, M.L., Brightwell, B.B., Schaefer, J., and Marvel, J.T., 1977, Metabolism and degradation of glyphosate in soil and water: Journal of Agricultural and Food Chemistry, v. 25 , no. 3 , p. 517-528.

Scribner, E.A., Battaglin, W.A., Dietze, J.E., and Thurman, E.M., 2003, Reconnaissance data for glyphosate, other selected herbicides, their degradation products, and antibiotics in 51 streams in nine Midwestern States, 2002: U.S. Geological Survey Open-File Report 03-217, 101 p.
Scribner, E.A., Battaglin, W.A., Goolsby, D.A., and Thurman, E.M., 2000, Changes in herbicide concentrations in Midwestern streams in relation to changes in use, 1989-1998: The Science of the Total Environment, v. 248, p. 255-263.

Scribner, E.A., Goolsby, D.A., Battaglin, W.A., Meyer, M.T., and Thurman, E.M., 2006, Concentrations of selected herbicides, herbicide degradation products, and nutrients in the lower Mississippi River, Louisiana, April 1991 through December 2003: U.S. Geological Survey Data Series 165, 84 p.

Scribner, E.A., Thurman, E.M., Goolsby, D.A., Meyer, M.T., Battaglin, W.A., and Kolpin, D.W., 2005, Summary of significant results from studies of triazine herbicides and their degradation products in surface water, ground water, and precipitation in the Midwestern United States during the 1990s: U.S. Geological Survey Scientific Investigations Report 2005-5094, 27 p.

Sprankle, P., Meggitt, W.F., and Penner, D, 1975, Adsorption, mobility, and microbial degradation of glyposate in the soil: Weed Science, v. 23, no. 3, p. 229-234.

Timme, P.J., 1995, National Water Quality Laboratory 1995 services catalog: U.S. Geological Survey Open-File Report 95-352, 92 p.

U.S. Department of Agriculture, 2006, National Agricultural Statistics Service (NASS) agricultural chemical use database: Information available on the Web, accessed February 6, 2006, at http://www.pestmanagement.info/nass/

U.S. Geological Survey, 2003, National Water-Quality Assessment Program (NAWQA): Information available on the Web, accessed November 4, 2003, at http://water.usgs. gov/nawqa

U.S. Geological Survey, 2006a, National Stream Quality Accounting Network (NASQAN): Information available on the Web, accessed April 9, 2007, at http://water.usgs. gov/nasqan/

U.S. Geological Survey, 2006b, National field manual for the collection of water-quality data: U.S. Geological Survey Techniques of Water-Resources Investigations, book 9, chap. A1-A9, [various pagination], available on the Web, accessed February 21, 2007, at http://pubs.water.usgs. gov/twri9A

Williams, G.M., Kroes, Robert, and Munro, I.C., 2000, Safety evaluation and risk assessment of the herbicide Roundup and its active ingredient, glyphosate, for humans: Regulatory Toxicology Pharmacology, v. 31, no. 2, p. 117-165. 
Data Tables 
Table 3. Analytical results for glyphosate, aminomethylphosphonic acid, and glufosinate in State cooperative surface- and ground-water studies, 2001-06.

[Sample type: FB, field blank; FR, field replicate; LD, laboratory duplicate; R, regular; --, no data; <, less than; $\mu \mathrm{g} / \mathrm{L}$, micrograms per liter]

\begin{tabular}{|c|c|c|c|c|c|c|c|}
\hline \multirow[b]{2}{*}{ Site number } & \multirow[b]{2}{*}{ Site location } & \multirow[b]{2}{*}{$\begin{array}{c}\text { Sam- } \\
\text { ple } \\
\text { type }\end{array}$} & \multirow[b]{2}{*}{$\begin{array}{c}\text { Date of } \\
\text { col- } \\
\text { lection } \\
\text { (month/ } \\
\text { day/year) }\end{array}$} & \multirow[b]{2}{*}{$\begin{array}{l}\text { Col- } \\
\text { lection } \\
\text { time } \\
\text { (24- } \\
\text { hour) }\end{array}$} & \multicolumn{3}{|c|}{ Concentrations } \\
\hline & & & & & $\begin{array}{l}\text { Gly- } \\
\text { pho- } \\
\text { sate } \\
\text { ( } \mu \mathrm{g} / \mathrm{L})\end{array}$ & $\begin{array}{c}\text { Amino- } \\
\text { meth- } \\
\text { ylphos- } \\
\text { phonic } \\
\text { acid } \\
\text { ( } \mathrm{\mu g} / \mathrm{L})\end{array}$ & $\begin{array}{c}\text { Glu- } \\
\text { fos- } \\
\text { inate } \\
\text { ( } \mu \mathrm{g} / \mathrm{L})\end{array}$ \\
\hline \multicolumn{8}{|c|}{ Florida Surface-Water Study (SKF) } \\
\hline \multirow[t]{2}{*}{02266923} & Swim Lake near Alcoma & $\mathrm{R}$ & $03 / 23 / 04$ & 1050 & $<0.02$ & $<0.02$ & $<0.02$ \\
\hline & & $\mathrm{R}$ & 06/08/04 & 1130 & $<.02$ & .06 & $<.02$ \\
\hline \multirow[t]{3}{*}{02270655} & Lake Lynn near DeSoto City & $\mathrm{R}$ & $03 / 22 / 04$ & 1050 & $<.02$ & $<.02$ & $<.02$ \\
\hline & & $\mathrm{LD}$ & $03 / 22 / 04$ & 1050 & $<.02$ & $<.02$ & $<.02$ \\
\hline & & $\mathrm{R}$ & 06/07/04 & 1010 & $<.02$ & .14 & $<.02$ \\
\hline \multirow[t]{3}{*}{02270700} & Lake Annie near Lake Placid & $\mathrm{R}$ & 03/23/05 & 1150 & $<.02$ & $<.02$ & $<.02$ \\
\hline & & $\mathrm{R}$ & $06 / 22 / 05$ & 1300 & $<.02$ & $<.02$ & $<.02$ \\
\hline & & $\mathrm{R}$ & 09/06/05 & 1400 & $<.02$ & $<.02$ & $<.02$ \\
\hline \multirow[t]{2}{*}{273323081290800} & Lake Denton near Avon Park & $\mathrm{R}$ & $03 / 22 / 04$ & 1440 & $<.02$ & $<.02$ & $<.02$ \\
\hline & & $\mathrm{R}$ & 06/07/04 & 1350 & $<.02$ & .06 & $<.02$ \\
\hline \multirow[t]{3}{*}{273850081310800} & Pabor Lake near Avon Park & $\mathrm{R}$ & $03 / 23 / 05$ & 0815 & $<.02$ & $<.02$ & $<.02$ \\
\hline & & $\mathrm{R}$ & $06 / 22 / 05$ & 0850 & $<.02$ & $<.02$ & $<.02$ \\
\hline & & $\mathrm{R}$ & 09/07/05 & 1300 & $<.02$ & $<.02$ & $<.02$ \\
\hline \multirow[t]{6}{*}{274648081315100} & Lake Moody near Frostproof & $\mathrm{R}$ & $03 / 22 / 05$ & 1215 & $<.02$ & $<.02$ & $<.02$ \\
\hline & & $\mathrm{R}$ & 03/22/05 & 1310 & $<.02$ & $<.02$ & $<.02$ \\
\hline & & $\mathrm{R}$ & $06 / 21 / 05$ & 1300 & $<.02$ & $<.02$ & $<.02$ \\
\hline & & FR & $06 / 21 / 05$ & 1400 & $<.02$ & $<.02$ & $<.02$ \\
\hline & & $\mathrm{R}$ & 09/06/05 & 0930 & $<.02$ & $<.02$ & $<.02$ \\
\hline & & $\mathrm{R}$ & 09/07/05 & 1215 & $<.02$ & $<.02$ & $<.02$ \\
\hline \multirow[t]{5}{*}{274744081304200} & Lake Leonore near Frostproof & $\mathrm{R}$ & $03 / 22 / 05$ & 0930 & $<.02$ & $<.02$ & $<.02$ \\
\hline & & $\mathrm{LD}$ & $03 / 22 / 05$ & 0930 & $<.02$ & $<.02$ & $<.02$ \\
\hline & & $\mathrm{R}$ & $06 / 21 / 05$ & 0900 & $<.02$ & $<.02$ & $<.02$ \\
\hline & & LD & $06 / 21 / 05$ & 0900 & $<.02$ & $<.02$ & $<.02$ \\
\hline & & $\mathrm{R}$ & 09/07/05 & 0850 & $<.02$ & $<.02$ & $<.02$ \\
\hline 275256081275900 & Lake Aurora at Hesperides & $\mathrm{R}$ & $03 / 23 / 04$ & 0805 & $<.02$ & $<.02$ & $<.02$ \\
\hline
\end{tabular}


Table 3. Analytical results for glyphosate, aminomethylphosphonic acid, and glufosinate in State cooperative surface- and ground-water studies, 2001-06.-Continued

[Sample type: FB, field blank; FR, field replicate; LD, laboratory duplicate; R, regular; --, no data; <, less than; $\mu \mathrm{g} / \mathrm{L}$, micrograms per liter]

\begin{tabular}{|c|c|c|c|c|c|c|c|}
\hline \multirow[b]{2}{*}{ Site number } & & \multirow[b]{2}{*}{$\begin{array}{c}\text { Sam- } \\
\text { ple } \\
\text { type }\end{array}$} & \multirow[b]{2}{*}{$\begin{array}{l}\text { Date of } \\
\text { col- } \\
\text { lection } \\
\text { (month/ } \\
\text { day/year) }\end{array}$} & \multirow[b]{2}{*}{$\begin{array}{c}\text { Col- } \\
\text { lection } \\
\text { time } \\
(24- \\
\text { hour) }\end{array}$} & \multicolumn{3}{|c|}{ Concentrations } \\
\hline & & & & & $\begin{array}{l}\text { Gly- } \\
\text { pho- } \\
\text { sate } \\
(\mu \mathrm{g} / \mathrm{L})\end{array}$ & $\begin{array}{c}\text { Amino- } \\
\text { meth- } \\
\text { ylphos- } \\
\text { phonic } \\
\text { acid } \\
(\mu \mathrm{g} / \mathrm{L})\end{array}$ & $\begin{array}{l}\text { Glu- } \\
\text { fos- } \\
\text { inate } \\
(\mu \mathrm{g} / \mathrm{L})\end{array}$ \\
\hline \multicolumn{8}{|c|}{ Florida Surface-Water Study (SKF)—Continued } \\
\hline 275256081275900 & Lake Aurora at Hesperides & $\mathrm{R}$ & 06/08/04 & 0815 & 0.02 & 0.08 & $<0.02$ \\
\hline \multicolumn{8}{|c|}{ Georgia Ground-Water Study (BGG) } \\
\hline \multirow[t]{2}{*}{311015084511901} & 07H021 & $\mathrm{R}$ & $11 / 14 / 01$ & 1500 & $<.10$ & $<.10$ & $<.10$ \\
\hline & & $\mathrm{R}$ & 06/19/02 & 1300 & $<.10$ & $<.10$ & $<.10$ \\
\hline \multirow[t]{2}{*}{310552084455601} & 08G008 & $\mathrm{R}$ & $11 / 15 / 01$ & 1100 & $<.10$ & $<.10$ & $<.10$ \\
\hline & & $\mathrm{R}$ & 06/19/02 & 1100 & $<.10$ & $<.10$ & $<.10$ \\
\hline \multirow[t]{3}{*}{312119084215601} & $11 \mathrm{~J} 021$ & $\mathrm{R}$ & $11 / 15 / 01$ & 1400 & $<.10$ & $<.10$ & $<.10$ \\
\hline & & $\mathrm{LD}$ & $11 / 15 / 01$ & 1400 & $<.10$ & $<.10$ & $<.10$ \\
\hline & & $\mathrm{R}$ & $06 / 19 / 02$ & 1600 & $<.10$ & $<.10$ & $<.10$ \\
\hline \multirow[t]{3}{*}{312908084151901} & $11 \mathrm{~K} 045$ & $\mathrm{R}$ & $11 / 16 / 01$ & 1000 & $<.10$ & $<.10$ & $<.10$ \\
\hline & & $\mathrm{LD}$ & $11 / 16 / 01$ & 1000 & $<.10$ & $<.10$ & $<.10$ \\
\hline & & $\mathrm{R}$ & $06 / 19 / 02$ & 1800 & $<.10$ & $<.10$ & $<.10$ \\
\hline \multirow[t]{4}{*}{320001084032801} & 13Q051 & $\mathrm{R}$ & $11 / 16 / 01$ & 1300 & $<.10$ & $<.10$ & $<.10$ \\
\hline & & $\mathrm{R}$ & 06/18/02 & 1500 & $<.10$ & $<.10$ & $<.10$ \\
\hline & & FR & 06/18/02 & 1500 & $<.10$ & $<.10$ & $<.10$ \\
\hline & & $\mathrm{LD}$ & 06/18/02 & 1502 & $<.10$ & $<.10$ & $<.10$ \\
\hline \multicolumn{8}{|c|}{ Illinois Ground-Water Study (EPI) } \\
\hline 421506088550001 & Illinois-BCCDGIS & $\mathrm{R}$ & 09/10/01 & 1708 & $<.10$ & $<.10$ & $<.10$ \\
\hline \multirow[t]{2}{*}{403143088054701} & Illinois-47583 & $\mathrm{R}$ & 10/09/01 & 1145 & $<.10$ & $<.10$ & $<.10$ \\
\hline & & LD & $10 / 09 / 01$ & 1145 & $<.10$ & $<.10$ & $<.10$ \\
\hline 403143088054701 & Illinois-00823 & $\mathrm{R}$ & $10 / 10 / 01$ & 1330 & $<.10$ & $<.10$ & $<.10$ \\
\hline 412603087452601 & Illinois-20458 & $\mathrm{R}$ & $10 / 10 / 01$ & 1440 & $<.10$ & $<.10$ & $<.10$ \\
\hline \multirow[t]{2}{*}{403432089380901} & Illinois-50060 & $\mathrm{R}$ & $10 / 15 / 01$ & 1047 & $<.10$ & $<.10$ & $<.10$ \\
\hline & & $\mathrm{LD}$ & $10 / 15 / 01$ & 1047 & $<.10$ & $<.10$ & $<.10$ \\
\hline 383932090012601 & Illinois-60053 & $\mathrm{R}$ & $10 / 16 / 01$ & 1126 & $<.10$ & $<.10$ & $<.10$ \\
\hline \multirow[t]{2}{*}{415551088575201} & Illinois-11782 & $\mathrm{R}$ & $10 / 23 / 01$ & 1000 & $<.10$ & $<.10$ & $<.10$ \\
\hline & & $\mathrm{LD}$ & 10/23/01 & 1000 & $<.10$ & $<.10$ & $<.10$ \\
\hline 404418089011802 & Illinois-31409 & $\mathrm{R}$ & 10/23/01 & 1240 & $<.10$ & $<.10$ & $<.10$ \\
\hline
\end{tabular}


Table 3. Analytical results for glyphosate, aminomethylphosphonic acid, and glufosinate in State cooperative surface- and ground-water studies, 2001-06.-Continued

[Sample type: FB, field blank; FR, field replicate; LD, laboratory duplicate; R, regular; --, no data; <, less than; $\mu \mathrm{g} / \mathrm{L}$, micrograms per liter]

\begin{tabular}{|c|c|c|c|c|c|c|c|}
\hline \multirow[b]{2}{*}{ Site number } & & \multirow[b]{2}{*}{ Site location } & \multirow[b]{2}{*}{$\begin{array}{c}\text { Date of } \\
\text { col- } \\
\text { lection } \\
\text { (month/ } \\
\text { day/year) }\end{array}$} & \multirow[b]{2}{*}{$\begin{array}{l}\text { Col- } \\
\text { lection } \\
\text { time } \\
(24- \\
\text { hour) }\end{array}$} & \multicolumn{3}{|c|}{ Concentrations } \\
\hline & & & & & $\begin{array}{l}\text { Gly- } \\
\text { pho- } \\
\text { sate } \\
\text { ( } \mu \mathrm{g} / \mathrm{L})\end{array}$ & $\begin{array}{l}\text { Amino- } \\
\text { meth- } \\
\text { ylphos- } \\
\text { phonic } \\
\text { acid } \\
\text { ( } \mu \mathrm{g} / \mathrm{L} \text { ) }\end{array}$ & $\begin{array}{c}\text { Glu- } \\
\text { fos- } \\
\text { inate } \\
\text { ( } \mu \mathrm{g} / \mathrm{L})\end{array}$ \\
\hline \multicolumn{8}{|c|}{ Illinois Ground-Water Study (EPI)_Continued } \\
\hline 404418089011802 & Illinois-31409 & $\mathrm{R}$ & $10 / 23 / 01$ & 1645 & $<0.10$ & $<0.10$ & $<0.10$ \\
\hline 403404089390701 & Illinois-50057 & $\mathrm{R}$ & $10 / 30 / 01$ & 1007 & $<.10$ & $<.10$ & $<.10$ \\
\hline 421433088002701 & Illinois-20250 & $\mathrm{R}$ & $10 / 31 / 01$ & 1115 & $<.10$ & $<.10$ & $<.10$ \\
\hline \multirow[t]{2}{*}{403116089251401} & Illinois-50055 & $\mathrm{R}$ & $10 / 30 / 01$ & 1235 & $<.10$ & $<.10$ & $<.10$ \\
\hline & & $\mathrm{R}$ & $10 / 30 / 01$ & 1310 & $<.10$ & $<.10$ & $<.10$ \\
\hline 413654088475601 & Illinois-11470 & $\mathrm{R}$ & $11 / 07 / 01$ & 1035 & $<.10$ & $<.10$ & $<.10$ \\
\hline 414319089203501 & Illinois-01079 & $\mathrm{R}$ & $11 / 07 / 01$ & 1250 & $<.10$ & $<.10$ & $<.10$ \\
\hline 421256088593801 & Illinois-11678 & $\mathrm{R}$ & 11/07/01 & 1445 & $<.10$ & $<.10$ & $<.10$ \\
\hline \multirow[t]{2}{*}{401754090032001} & Illinois-50308 & $\mathrm{R}$ & 11/13/01 & 1035 & $<.10$ & $<.10$ & $<.10$ \\
\hline & & $\mathrm{LD}$ & $11 / 13 / 01$ & 1035 & $<.10$ & $<.10$ & $<.10$ \\
\hline 422225088102501 & Illinois-02305 & $\mathrm{R}$ & $11 / 20 / 01$ & 955 & $<.10$ & $<.10$ & $<.10$ \\
\hline 421840088033001 & Illinois-00366 & $\mathrm{R}$ & $11 / 20 / 01$ & 1100 & $<.10$ & $<.10$ & $<.10$ \\
\hline 412608088121401 & Illinois-20357 & $\mathrm{R}$ & $11 / 21 / 01$ & 1320 & $<.10$ & $<.10$ & $<.10$ \\
\hline 404625087335301 & Illinois-47561 & $\mathrm{R}$ & $11 / 27 / 01$ & 1110 & $<.10$ & $<.10$ & $<.10$ \\
\hline \multirow[t]{2}{*}{411928089073001} & Illinois-11509 & $\mathrm{R}$ & $12 / 04 / 01$ & 1030 & $<.10$ & $<.10$ & $<.10$ \\
\hline & & FR & $12 / 04 / 01$ & 1031 & $<.10$ & $<.10$ & $<.10$ \\
\hline 400112090312301 & Illinois-01118 & $\mathrm{R}$ & $12 / 04 / 01$ & 1115 & $<.10$ & $<.10$ & $<.10$ \\
\hline \multirow[t]{3}{*}{410002088313401} & Illinois- 47518 & $\mathrm{R}$ & $12 / 04 / 01$ & 1320 & $<.10$ & $<.10$ & $<.10$ \\
\hline & & LD & $12 / 04 / 01$ & 1320 & $<.10$ & $<.10$ & $<.10$ \\
\hline & & FR & $12 / 04 / 01$ & 1321 & $<.10$ & $<.10$ & $<.10$ \\
\hline 421222088152701 & Illinois-20141 & $\mathrm{R}$ & $12 / 09 / 01$ & 0955 & $<.10$ & $<.10$ & $<.10$ \\
\hline 422219088222601 & Illinois-00595 & $\mathrm{R}$ & $12 / 09 / 01$ & 1120 & $<.10$ & $<.10$ & $<.10$ \\
\hline-- & Illinois-11891 & $\mathrm{R}$ & $12 / 11 / 01$ & 1110 & $<.10$ & $<.10$ & $<.10$ \\
\hline-- & Illinois-20330 & $\mathrm{R}$ & $12 / 11 / 01$ & 1230 & $<.10$ & $<.10$ & $<.10$ \\
\hline 403737089365401 & Illinois-50376 & $\mathrm{R}$ & $12 / 12 / 01$ & 1055 & $<.10$ & $<.10$ & $<.10$ \\
\hline \multirow[t]{2}{*}{--} & Illinois-50251 & $\mathrm{R}$ & $12 / 12 / 01$ & 1315 & $<.10$ & $<.10$ & $<.10$ \\
\hline & & FR & $12 / 12 / 01$ & 1317 & $<.10$ & $<.10$ & $<.10$ \\
\hline \multirow[t]{2}{*}{404417090360801} & Illinois-50094 & $\mathrm{R}$ & $12 / 18 / 01$ & 1155 & $<.10$ & $<.10$ & $<.10$ \\
\hline & & FR & $12 / 18 / 01$ & 1157 & $<.10$ & $<.10$ & $<.10$ \\
\hline
\end{tabular}


Table 3. Analytical results for glyphosate, aminomethylphosphonic acid, and glufosinate in State cooperative surface- and ground-water studies, 2001-06.-Continued

[Sample type: FB, field blank; FR, field replicate; LD, laboratory duplicate; R, regular; --, no data; <, less than; $\mu \mathrm{g} / \mathrm{L}$, micrograms per liter]

\begin{tabular}{|c|c|c|c|c|c|c|c|}
\hline \multirow[b]{2}{*}{ Site number } & & \multirow[b]{2}{*}{ Site location } & \multirow[b]{2}{*}{$\begin{array}{c}\text { Date of } \\
\text { col-- } \\
\text { lection } \\
\text { (month/ } \\
\text { day/year) }\end{array}$} & \multirow[b]{2}{*}{$\begin{array}{c}\text { Col- } \\
\text { lection } \\
\text { time } \\
\text { (24- } \\
\text { hour) }\end{array}$} & \multicolumn{3}{|c|}{ Concentrations } \\
\hline & & & & & $\begin{array}{l}\text { Gly- } \\
\text { pho- } \\
\text { sate } \\
\text { ( } \mu \mathrm{g} / \mathrm{L})\end{array}$ & $\begin{array}{c}\text { Amino- } \\
\text { meth- } \\
\text { ylphos- } \\
\text { phonic } \\
\text { acid } \\
(\mu \mathrm{g} / \mathrm{L}) \\
\end{array}$ & $\begin{array}{l}\text { Glu- } \\
\text { fos- } \\
\text { inate } \\
(\mu \mathrm{g} / \mathrm{L})\end{array}$ \\
\hline \multicolumn{8}{|c|}{ Illinois Ground-Water Study (EPI)—Continued } \\
\hline 385946088020701 & Illinois-47819 & $\mathrm{R}$ & $12 / 19 / 01$ & 1130 & $<0.10$ & $<0.10$ & $<0.10$ \\
\hline 394602090360801 & Illinois-52121 & $\mathrm{R}$ & $01 / 09 / 02$ & 1045 & $<.10$ & $<.10$ & $<.10$ \\
\hline 394605089155301 & Illinois-50224 & $\mathrm{R}$ & $01 / 09 / 02$ & 1425 & $<.10$ & $<.10$ & $<.10$ \\
\hline \multirow[t]{2}{*}{421803087554801} & Illinois-20285 & $\mathrm{R}$ & $01 / 14 / 02$ & 1055 & $<.10$ & $<.10$ & $<.10$ \\
\hline & & $\mathrm{LD}$ & $01 / 14 / 02$ & 1055 & $<.10$ & $<.10$ & $<.10$ \\
\hline \multirow[t]{2}{*}{404532088111201} & Illinois-47532 & $\mathrm{R}$ & $01 / 15 / 02$ & 0950 & $<.10$ & $<.10$ & $<.10$ \\
\hline & & FR & $01 / 15 / 02$ & 0952 & $<.10$ & $<.10$ & $<.10$ \\
\hline 404614087512502 & Illinois-47551 & $\mathrm{R}$ & $01 / 15 / 02$ & 1150 & $<.10$ & $<.10$ & $<.10$ \\
\hline 372000088431001 & Illinois-00757 & $\mathrm{R}$ & $01 / 22 / 02$ & 1340 & $<.10$ & $<.10$ & $<.10$ \\
\hline 415410088034301 & Illinois-20700 & $\mathrm{R}$ & $01 / 23 / 02$ & 1020 & $<.10$ & $<.10$ & $<.10$ \\
\hline 410134089244503 & Illinois-31307 & $\mathrm{R}$ & $01 / 29 / 02$ & 1110 & $<.10$ & $<.10$ & $<.10$ \\
\hline 405404089022301 & Illinois-31428 & $\mathrm{R}$ & $01 / 29 / 02$ & 1310 & $<.10$ & $<.10$ & $<.10$ \\
\hline 421611089031101 & Illinois-11636 & $\mathrm{R}$ & $02 / 04 / 02$ & 1050 & $<.10$ & $<.10$ & $<.10$ \\
\hline 415035089175901 & Illinois-11562 & $\mathrm{R}$ & $02 / 04 / 02$ & 1400 & $<.10$ & $<.10$ & $<.10$ \\
\hline 415552088465601 & Illinois-11406 & $\mathrm{R}$ & $02 / 05 / 02$ & 0830 & $<.10$ & $<.10$ & $<.10$ \\
\hline 413805088405102 & Illinois-11438 & $\mathrm{R}$ & $02 / 05 / 02$ & 1000 & $<.10$ & $<.10$ & $<.10$ \\
\hline-- & Illinois-20497 & $\mathrm{R}$ & $02 / 11 / 02$ & 1317 & $<.10$ & $<.10$ & $<.10$ \\
\hline 395411087485701 & Illinois-47692 & $\mathrm{R}$ & $02 / 19 / 02$ & 1330 & $<.10$ & $<.10$ & $<.10$ \\
\hline 381238090160001 & Illinois-60127 & $\mathrm{R}$ & $02 / 25 / 02$ & 1205 & $<.10$ & $<.10$ & $<.10$ \\
\hline \multirow[t]{3}{*}{390644087391802} & Illinois-47811 & $\mathrm{R}$ & 03/04/02 & 1035 & $<.10$ & $<.10$ & $<.10$ \\
\hline & & $\mathrm{LD}$ & 03/04/02 & 1035 & $<.10$ & $<.10$ & $<.10$ \\
\hline & & FR & 03/04/02 & 1300 & $<.10$ & $<.10$ & $<.10$ \\
\hline 391251087393801 & Illinois-00251 & $\mathrm{R}$ & 03/04/02 & 1200 & $<.10$ & $<.10$ & $<.10$ \\
\hline \multirow[t]{2}{*}{412534089550901} & Illinois-11347 & $\mathrm{R}$ & 03/05/02 & 1110 & $<.10$ & $<.10$ & $<.10$ \\
\hline & & FR & 03/05/02 & 1111 & $<.10$ & $<.10$ & $<.10$ \\
\hline \multirow[t]{2}{*}{412534089215601} & Illinois-11327 & $\mathrm{R}$ & 03/05/02 & 1230 & $<.10$ & $<.10$ & $<.10$ \\
\hline & & $\mathrm{R}$ & 03/05/02 & 1530 & $<.10$ & $<.10$ & $<.10$ \\
\hline 414305089550901 & Illinois-11904 & $\mathrm{R}$ & 03/06/02 & 1130 & $<.10$ & $<.10$ & $<.10$ \\
\hline 420559088322101 & Illinois-00795 & $\mathrm{R}$ & 03/11/02 & 0930 & $<.10$ & $<.10$ & $<.10$ \\
\hline 395640088515501 & Illinois-47725 & $\mathrm{R}$ & 03/12/02 & 0955 & $<.10$ & $<.10$ & $<.10$ \\
\hline
\end{tabular}


Table 3. Analytical results for glyphosate, aminomethylphosphonic acid, and glufosinate in State cooperative surface- and ground-water studies, 2001-06.-Continued

[Sample type: FB, field blank; FR, field replicate; LD, laboratory duplicate; R, regular; --, no data; <, less than; $\mu \mathrm{g} / \mathrm{L}$, micrograms per liter]

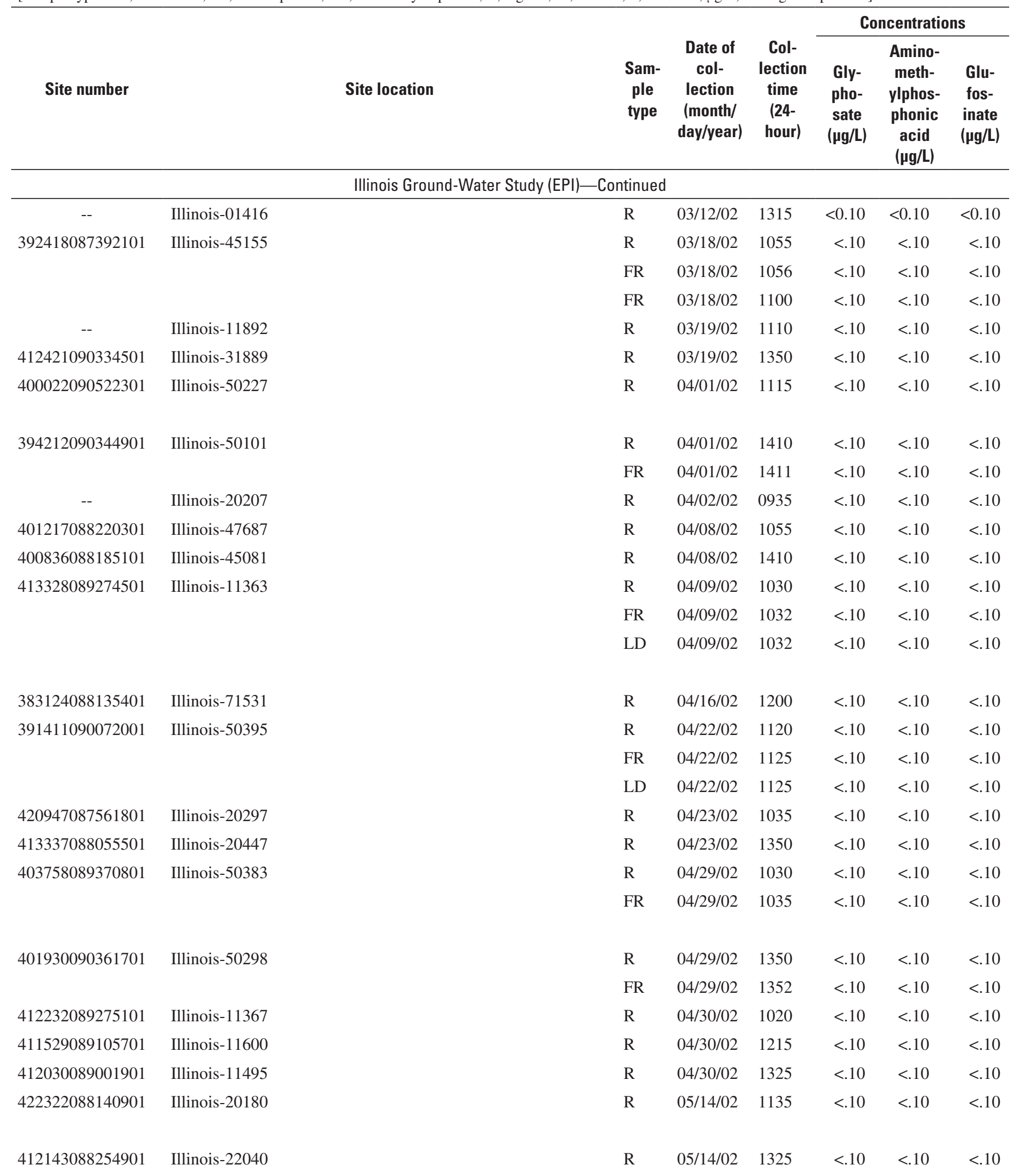


Table 3. Analytical results for glyphosate, aminomethylphosphonic acid, and glufosinate in State cooperative surface- and ground-water studies, 2001-06.-Continued

[Sample type: FB, field blank; FR, field replicate; LD, laboratory duplicate; R, regular; --, no data; <, less than; $\mu \mathrm{g} / \mathrm{L}$, micrograms per liter]

\begin{tabular}{|c|c|c|c|c|c|c|c|}
\hline \multirow[b]{2}{*}{ Site number } & \multirow[b]{2}{*}{ Site location } & \multirow[b]{2}{*}{$\begin{array}{c}\text { Sam- } \\
\text { ple } \\
\text { type }\end{array}$} & \multirow[b]{2}{*}{$\begin{array}{c}\text { Date of } \\
\text { col- } \\
\text { lection } \\
\text { (month/ } \\
\text { day/year) }\end{array}$} & \multirow[b]{2}{*}{$\begin{array}{c}\text { Col- } \\
\text { lection } \\
\text { time } \\
(24- \\
\text { hour) }\end{array}$} & \multicolumn{3}{|c|}{ Concentrations } \\
\hline & & & & & $\begin{array}{l}\text { Gly- } \\
\text { pho- } \\
\text { sate } \\
(\mu \mathrm{g} / \mathrm{L})\end{array}$ & $\begin{array}{l}\text { Amino- } \\
\text { meth- } \\
\text { ylphos- } \\
\text { phonic } \\
\text { acid } \\
(\mu \mathrm{g} / \mathrm{L})\end{array}$ & $\begin{array}{c}\text { Glu- } \\
\text { fos- } \\
\text { inate } \\
(\mu \mathrm{g} / \mathrm{L})\end{array}$ \\
\hline \multicolumn{8}{|c|}{ Illinois Ground-Water Study (EPI)—Continued } \\
\hline 412143088254901 & Illinois-22040 & $\mathrm{R}$ & 05/14/02 & 1425 & $<0.10$ & $<0.10$ & $<0.10$ \\
\hline \multirow[t]{2}{*}{421359088323201} & Illinois-00276 & $\mathrm{R}$ & $05 / 20 / 02$ & 0800 & $<.10$ & $<.10$ & $<.10$ \\
\hline & & FR & 05/20/02 & 0802 & $<.10$ & $<.10$ & $<.10$ \\
\hline 415027089175501 & Illinois-11560 & $\mathrm{R}$ & $05 / 20 / 02$ & 1030 & $<.10$ & $<.10$ & $<.10$ \\
\hline \multirow[t]{2}{*}{49332088363201} & Illinois-00572 & $\mathrm{R}$ & $05 / 21 / 02$ & 1035 & $<.10$ & $<.10$ & $<.10$ \\
\hline & & FR & $05 / 21 / 02$ & 1040 & $<.10$ & $<.10$ & $<.10$ \\
\hline 392439089262001 & Illinois-50237 & $\mathrm{R}$ & $05 / 21 / 02$ & 1335 & $<.10$ & $<.10$ & $<.10$ \\
\hline 394338090542001 & Illinois-52062 & $\mathrm{R}$ & 06/05/02 & 1130 & $<.10$ & $<.10$ & $<.10$ \\
\hline 415839088113901 & Illinois-20613 & $\mathrm{R}$ & $06 / 11 / 02$ & 0950 & $<.10$ & $<.10$ & $<.10$ \\
\hline 404511090004001 & Illinois-50351 & $\mathrm{R}$ & $06 / 11 / 02$ & 1425 & $<.10$ & $<.10$ & $<.10$ \\
\hline 412854087503501 & Illinois-20442 & $\mathrm{R}$ & $06 / 17 / 02$ & 1335 & $<.10$ & $<.10$ & $<.10$ \\
\hline 403551088021302 & Illinois-47573 & $\mathrm{R}$ & $06 / 17 / 02$ & 1120 & $<.10$ & $<.10$ & $<.10$ \\
\hline 421656089031301 & Illinois-11629 & $\mathrm{R}$ & 06/18/02 & 0905 & $<.10$ & $<.10$ & $<.10$ \\
\hline 414955089291901 & Illinois-11557 & $\mathrm{R}$ & $06 / 18 / 02$ & 1125 & $<.10$ & $<.10$ & $<.10$ \\
\hline 420727088153901 & Illinois-20029 & $\mathrm{R}$ & $06 / 24 / 02$ & 0950 & $<.10$ & $<.10$ & $<.10$ \\
\hline 4155090089032801 & Illinois-11943 & $\mathrm{R}$ & $06 / 25 / 02$ & 1005 & $<.10$ & $<.10$ & $<.10$ \\
\hline 414122088585401 & Illinois-11570 & $\mathrm{R}$ & $06 / 25 / 02$ & 1130 & $<.10$ & $<.10$ & $<.10$ \\
\hline 414945088115101 & Illinois-20766 & $\mathrm{R}$ & 07/01/02 & 1255 & $<.10$ & $<.10$ & $<.10$ \\
\hline \multirow[t]{2}{*}{395106089432801} & Illinois-50003 & $\mathrm{R}$ & 07/02/02 & 1040 & $<.10$ & $<.10$ & $<.10$ \\
\hline & & FR & 07/02/02 & 1045 & $<.10$ & $<.10$ & $<.10$ \\
\hline 385235090072601 & Illinois-60058 & $\mathrm{R}$ & $07 / 02 / 02$ & 1055 & $<.10$ & $<.10$ & $<.10$ \\
\hline 395106089432801 & Illinois-50003 & $\mathrm{R}$ & 07/02/02 & 1040 & $<.10$ & $<.10$ & $<.10$ \\
\hline 404126089305001 & Illinois-50051 & $\mathrm{R}$ & $07 / 17 / 02$ & 1325 & $<.10$ & $<.10$ & $<.10$ \\
\hline \multirow[t]{2}{*}{420608089501401} & Illinois-11707 & $\mathrm{R}$ & 07/29/02 & 1030 & $<.10$ & $<.10$ & $<.10$ \\
\hline & & $\mathrm{LD}$ & 07/29/02 & 1030 & $<.10$ & $<.10$ & $<.10$ \\
\hline 410931088384401 & Illinois-11514 & $\mathrm{R}$ & $07 / 30 / 02$ & 1300 & $<.10$ & $<.10$ & $<.10$ \\
\hline \multicolumn{8}{|c|}{ lowa Surface-Water Study (DJS) } \\
\hline \multirow[t]{2}{*}{05418500} & \multirow[t]{2}{*}{ Maquoketa River near Maquoketa } & $\mathrm{R}$ & $06 / 05 / 02$ & 1200 & $<.10$ & .18 & $<.10$ \\
\hline & & $\mathrm{LD}$ & 06/05/02 & 1200 & $<.10$ & .17 & $<.10$ \\
\hline 05420500 & Mississippi River at Clinton & $\mathrm{R}$ & $05 / 23 / 03$ & 1040 & $<.10$ & $<.10$ & $<.10$ \\
\hline 05422000 & Wapsipinicon River near DeWitt & $\mathrm{R}$ & 06/05/02 & 1430 & $<.10$ & .22 & $<.10$ \\
\hline
\end{tabular}


Table 3. Analytical results for glyphosate, aminomethylphosphonic acid, and glufosinate in State cooperative surface- and ground-water studies, 2001-06.-Continued

[Sample type: FB, field blank; FR, field replicate; LD, laboratory duplicate; R, regular; --, no data; <, less than; $\mu \mathrm{g} / \mathrm{L}$, micrograms per liter]

\begin{tabular}{|c|c|c|c|c|c|c|c|}
\hline \multirow[b]{2}{*}{ Site number } & \multirow[b]{2}{*}{ Site location } & \multirow[b]{2}{*}{$\begin{array}{c}\text { Sam- } \\
\text { ple } \\
\text { type }\end{array}$} & \multirow[b]{2}{*}{$\begin{array}{c}\text { Date of } \\
\text { col- } \\
\text { lection } \\
\text { (month/ } \\
\text { day/year) }\end{array}$} & \multirow[b]{2}{*}{$\begin{array}{c}\text { Col- } \\
\text { lection } \\
\text { time } \\
\text { (24- } \\
\text { hour) }\end{array}$} & \multicolumn{3}{|c|}{ Concentrations } \\
\hline & & & & & $\begin{array}{l}\text { Gly- } \\
\text { pho- } \\
\text { sate } \\
\text { ( } \mu \text { g/L) }\end{array}$ & $\begin{array}{c}\text { Amino- } \\
\text { meth- } \\
\text { ylphos- } \\
\text { phonic } \\
\text { acid } \\
\text { ( } \mathrm{rg} / \mathrm{L} \text { ) }\end{array}$ & $\begin{array}{c}\text { Glu- } \\
\text { fos- } \\
\text { inate } \\
\text { ( } \mu \mathrm{g} / \mathrm{L} \text { ) }\end{array}$ \\
\hline \multicolumn{8}{|c|}{ lowa Surface-Water Study (DJS) —Continued } \\
\hline 05457470 & Cedar River at St. Ansgar & $\mathrm{R}$ & 09/23/02 & 1058 & 0.47 & 0.81 & $<0.10$ \\
\hline 05458500 & Cedar River at Janesville & $\mathrm{R}$ & $09 / 24 / 02$ & 0752 & $<.10$ & .41 & $<.10$ \\
\hline 05458900 & West Fork Cedar River at Finchford & $\mathrm{R}$ & $09 / 24 / 02$ & 0950 & $<.10$ & .26 & $<.10$ \\
\hline 05462000 & Shell Rock River at Shell Rock & $\mathrm{R}$ & $09 / 23 / 02$ & 1705 & $<.10$ & .44 & $<.10$ \\
\hline 05464090 & Cedar River near LaPorte City & $\mathrm{R}$ & $09 / 25 / 02$ & 0905 & $<.10$ & .27 & $<.10$ \\
\hline 05464220 & Wolf Creek near Dysart & $\mathrm{R}$ & $09 / 24 / 02$ & 1410 & $<.10$ & .21 & $<.10$ \\
\hline \multirow[t]{2}{*}{05464420} & Cedar River at Blairs Ferry Road at Palo & $\mathrm{R}$ & $09 / 23 / 02$ & 1415 & $<.10$ & .28 & $<.10$ \\
\hline & & $\mathrm{LD}$ & $09 / 23 / 02$ & 1415 & $<.10$ & .26 & $<.10$ \\
\hline \multirow[t]{2}{*}{05464480} & Cedar River at Edgewood Road at Cedar Rapids & $\mathrm{R}$ & 09/04/02 & 0915 & $<.10$ & $<.10$ & $<.10$ \\
\hline & & $\mathrm{LD}$ & 09/04/02 & 0915 & $<.10$ & $<.10$ & $<.10$ \\
\hline 122 & -- & $\mathrm{R}$ & $06 / 14 / 03$ & 1015 & $<.10$ & $<.10$ & $<.10$ \\
\hline 123 & -- & $\mathrm{R}$ & 06/14/03 & 1000 & $<.10$ & $<.10$ & $<.10$ \\
\hline 124 & -- & $\mathrm{R}$ & $06 / 14 / 03$ & 0945 & $<.10$ & $<.10$ & $<.10$ \\
\hline 124 & -- & LD & 06/14/03 & 0945 & $<.10$ & $<.10$ & $<.10$ \\
\hline 126 & -- & $\mathrm{R}$ & $06 / 14 / 03$ & 0920 & $<.10$ & $<.10$ & $<.10$ \\
\hline 164 & -- & $\mathrm{R}$ & $06 / 14 / 03$ & 1058 & $<.10$ & .16 & $<.10$ \\
\hline 164 & -- & LD & $06 / 14 / 03$ & 1058 & $<.10$ & .16 & $<.10$ \\
\hline 192 & -- & $\mathrm{R}$ & 06/14/03 & 1025 & $<.10$ & .18 & $<.10$ \\
\hline 341 & -- & $\mathrm{R}$ & $06 / 14 / 03$ & 0935 & $<.10$ & .14 & $<.10$ \\
\hline 351 & -- & $\mathrm{R}$ & $06 / 14 / 03$ & 1045 & $<.10$ & $<.10$ & $<.10$ \\
\hline 352 & -- & $\mathrm{R}$ & $06 / 14 / 03$ & 1030 & $<.10$ & $<.10$ & $<.10$ \\
\hline 381 & -- & $\mathrm{R}$ & 06/14/03 & 1018 & $<.10$ & $<.10$ & $<.10$ \\
\hline 382 & -- & $\mathrm{R}$ & $06 / 14 / 03$ & 0954 & $<.10$ & $<.10$ & $<.10$ \\
\hline 383 & -- & $\mathrm{R}$ & $06 / 14 / 03$ & 1045 & $<.10$ & $<.10$ & $<.10$ \\
\hline 384 & -- & $\mathrm{R}$ & $06 / 14 / 03$ & 0910 & $<.10$ & $<.10$ & $<.10$ \\
\hline 573 & -- & $\mathrm{R}$ & $06 / 14 / 03$ & 1140 & $<.10$ & $<.10$ & $<.10$ \\
\hline 574 & -- & $\mathrm{R}$ & $06 / 14 / 03$ & 1215 & $<.10$ & $<.10$ & $<.10$ \\
\hline 61 & -- & $\mathrm{R}$ & $06 / 14 / 03$ & 1325 & $<.10$ & $<.10$ & $<.10$ \\
\hline 62 & -- & $\mathrm{R}$ & $06 / 14 / 03$ & 0730 & $<.10$ & $<.10$ & $<.10$ \\
\hline 662 & -- & $\mathrm{R}$ & $06 / 14 / 03$ & 0801 & 3.5 & .68 & $<.10$ \\
\hline
\end{tabular}


Table 3. Analytical results for glyphosate, aminomethylphosphonic acid, and glufosinate in State cooperative surface- and ground-water studies, 2001-06.-Continued

[Sample type: FB, field blank; FR, field replicate; LD, laboratory duplicate; R, regular; --, no data; <, less than; $\mu \mathrm{g} / \mathrm{L}$, micrograms per liter]

\begin{tabular}{|c|c|c|c|c|c|c|c|c|}
\hline \multirow[b]{2}{*}{ Site number } & \multirow{2}{*}{\multicolumn{2}{|c|}{ Site location }} & \multirow[b]{2}{*}{$\begin{array}{c}\text { Sam- } \\
\text { ple } \\
\text { type }\end{array}$} & \multirow[b]{2}{*}{$\begin{array}{c}\text { Date of } \\
\text { col- } \\
\text { lection } \\
\text { (month/ } \\
\text { day/year) }\end{array}$} & \multirow[b]{2}{*}{$\begin{array}{c}\text { Col- } \\
\text { lection } \\
\text { time } \\
(24- \\
\text { hour) }\end{array}$} & \multicolumn{3}{|c|}{ Concentrations } \\
\hline & & & & & & $\begin{array}{c}\text { Gly- } \\
\text { pho- } \\
\text { sate } \\
(\mu \mathrm{g} / \mathrm{L})\end{array}$ & $\begin{array}{c}\text { Amino- } \\
\text { meth- } \\
\text { ylphos- } \\
\text { phonic } \\
\text { acid } \\
\text { ( } \mu \mathrm{g} / \mathrm{L})\end{array}$ & $\begin{array}{l}\text { Glu- } \\
\text { fos- } \\
\text { inate } \\
\text { ( } \mu \mathrm{g} / \mathrm{L})\end{array}$ \\
\hline \multicolumn{9}{|c|}{ lowa Surface-Water Study (DJS)—Continued } \\
\hline 701 & & -- & $\mathrm{R}$ & $06 / 14 / 03$ & 0857 & $<0.10$ & $<0.10$ & $<0.10$ \\
\hline 702 & & -- & $\mathrm{R}$ & $06 / 14 / 03$ & 0812 & $<.10$ & .19 & $<.10$ \\
\hline 704 & & -- & $\mathrm{R}$ & $06 / 14 / 03$ & 0933 & $<.10$ & .13 & $<.10$ \\
\hline 71 & & -- & $\mathrm{R}$ & $06 / 14 / 03$ & 0930 & $<.10$ & .10 & $<.10$ \\
\hline 73 & & -- & $\mathrm{R}$ & $06 / 14 / 03$ & 0800 & $<.10$ & $<.10$ & $<.10$ \\
\hline 74 & & -- & $\mathrm{R}$ & $06 / 14 / 03$ & 1000 & $<.10$ & .13 & $<.10$ \\
\hline 77 & & -- & $\mathrm{R}$ & 06/14/03 & 1000 & $<.10$ & $<.10$ & $<.10$ \\
\hline 861 & & -- & $\mathrm{R}$ & $06 / 14 / 03$ & 1215 & $<.10$ & $<.10$ & $<.10$ \\
\hline 862 & & -- & $\mathrm{R}$ & 06/14/03 & 1115 & $<.10$ & $<.10$ & $<.10$ \\
\hline 865 & & -- & $\mathrm{R}$ & $06 / 14 / 03$ & 1200 & $<.10$ & $<.10$ & $<.10$ \\
\hline 93 & & -- & $\mathrm{R}$ & 06/14/03 & 0820 & $<.10$ & .15 & $<.10$ \\
\hline \multicolumn{9}{|c|}{ lowa Ground-Water Study (DJS) } \\
\hline 415949091405401 & CRM-1 & & $\mathrm{R}$ & 09/05/02 & 1330 & $<.10$ & $<.10$ & $<.10$ \\
\hline 415953091435301 & CRM-4 & & $\mathrm{R}$ & 09/05/02 & 0930 & $<.10$ & $<.10$ & $<.10$ \\
\hline \multirow[t]{2}{*}{420009091432101} & CRM-27 & & $\mathrm{R}$ & 09/03/02 & 1400 & $<.10$ & $<.10$ & $<.10$ \\
\hline & & & $\mathrm{R}$ & 09/04/02 & 1715 & $<.10$ & $<.10$ & $<.10$ \\
\hline 420012091432601 & CRM-25 & & $\mathrm{R}$ & 09/03/02 & 1200 & $<.10$ & $<.10$ & $<.10$ \\
\hline 420013091431001 & CRM-22 & & $\mathrm{R}$ & 09/04/02 & 1045 & $<.10$ & $<.10$ & $<.10$ \\
\hline 420015091431301 & CRM-23 & & $\mathrm{R}$ & 09/04/02 & 1315 & $<.10$ & $<.10$ & $<.10$ \\
\hline \multirow[t]{2}{*}{420018091430801} & CRM-26 & & $\mathrm{R}$ & 09/04/02 & 1530 & $<.10$ & $<.10$ & $<.10$ \\
\hline & & & FR & 09/04/02 & 1535 & $<.10$ & $<.10$ & $<.10$ \\
\hline 420033091420301 & CRM-2 & & $\mathrm{R}$ & 09/05/02 & 1130 & $<.10$ & $<.10$ & $<.10$ \\
\hline 420002091403200 & Water treatment plant & & $\mathrm{R}$ & $09 / 05 / 02$ & 1515 & $<.10$ & $<.10$ & $<.10$ \\
\hline 420010091431801 & Seminole Ranney & & $\mathrm{R}$ & $05 / 29 / 03$ & 1315 & $<.10$ & $<.10$ & $<.10$ \\
\hline 420035091422301 & Edgewood Ranney & & $\mathrm{R}$ & $05 / 29 / 03$ & 1400 & $<.10$ & $<.10$ & $<.10$ \\
\hline \multicolumn{9}{|c|}{ lowa Ground-Water Study (DKW) } \\
\hline-- & Fairfield $94-1$ & & $\mathrm{R}$ & 09/06/01 & 1000 & $<.10$ & $<.10$ & $<.10$ \\
\hline \multirow[t]{2}{*}{403659094285301} & Blockton \#1 & & $\mathrm{R}$ & $08 / 14 / 01$ & 0915 & $<.10$ & $<.10$ & $<.10$ \\
\hline & & & LD & $08 / 14 / 01$ & 0915 & $<.10$ & $<.10$ & $<.10$ \\
\hline \multirow[t]{2}{*}{403745091174701} & Fort Madison 4 & & $\mathrm{R}$ & 09/06/01 & 1300 & $<.10$ & $<.10$ & $<.10$ \\
\hline & & & LD & 09/06/01 & 1300 & $<.10$ & $<.10$ & $<.10$ \\
\hline
\end{tabular}


Table 3. Analytical results for glyphosate, aminomethylphosphonic acid, and glufosinate in State cooperative surface- and ground-water studies, 2001-06.-Continued

[Sample type: FB, field blank; FR, field replicate; LD, laboratory duplicate; R, regular; --, no data; <, less than; $\mu \mathrm{g} / \mathrm{L}$, micrograms per liter]

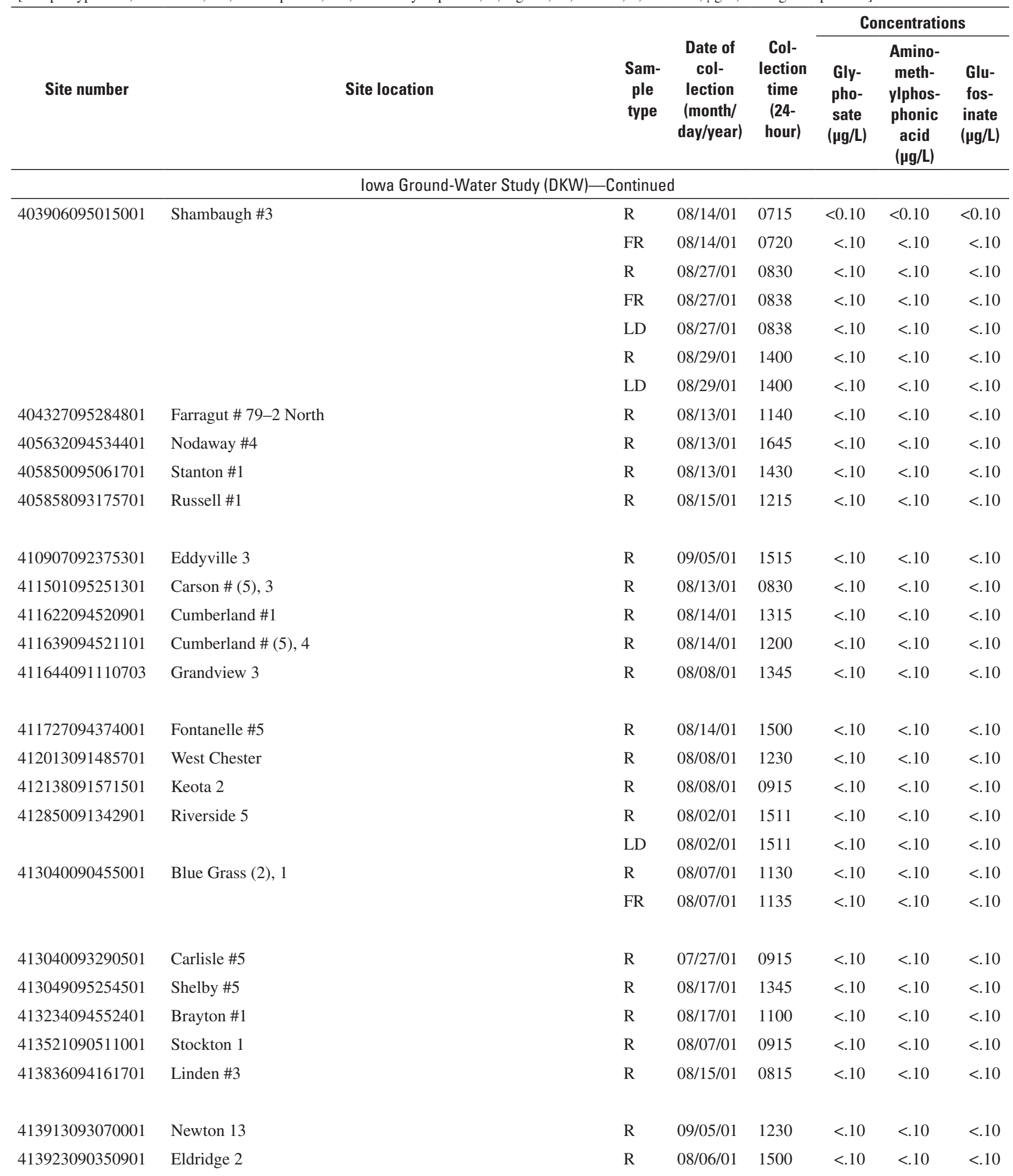


Table 3. Analytical results for glyphosate, aminomethylphosphonic acid, and glufosinate in State cooperative surface- and ground-water studies, 2001-06.-Continued

[Sample type: FB, field blank; FR, field replicate; LD, laboratory duplicate; R, regular; --, no data; <, less than; $\mu \mathrm{g} / \mathrm{L}$, micrograms per liter]

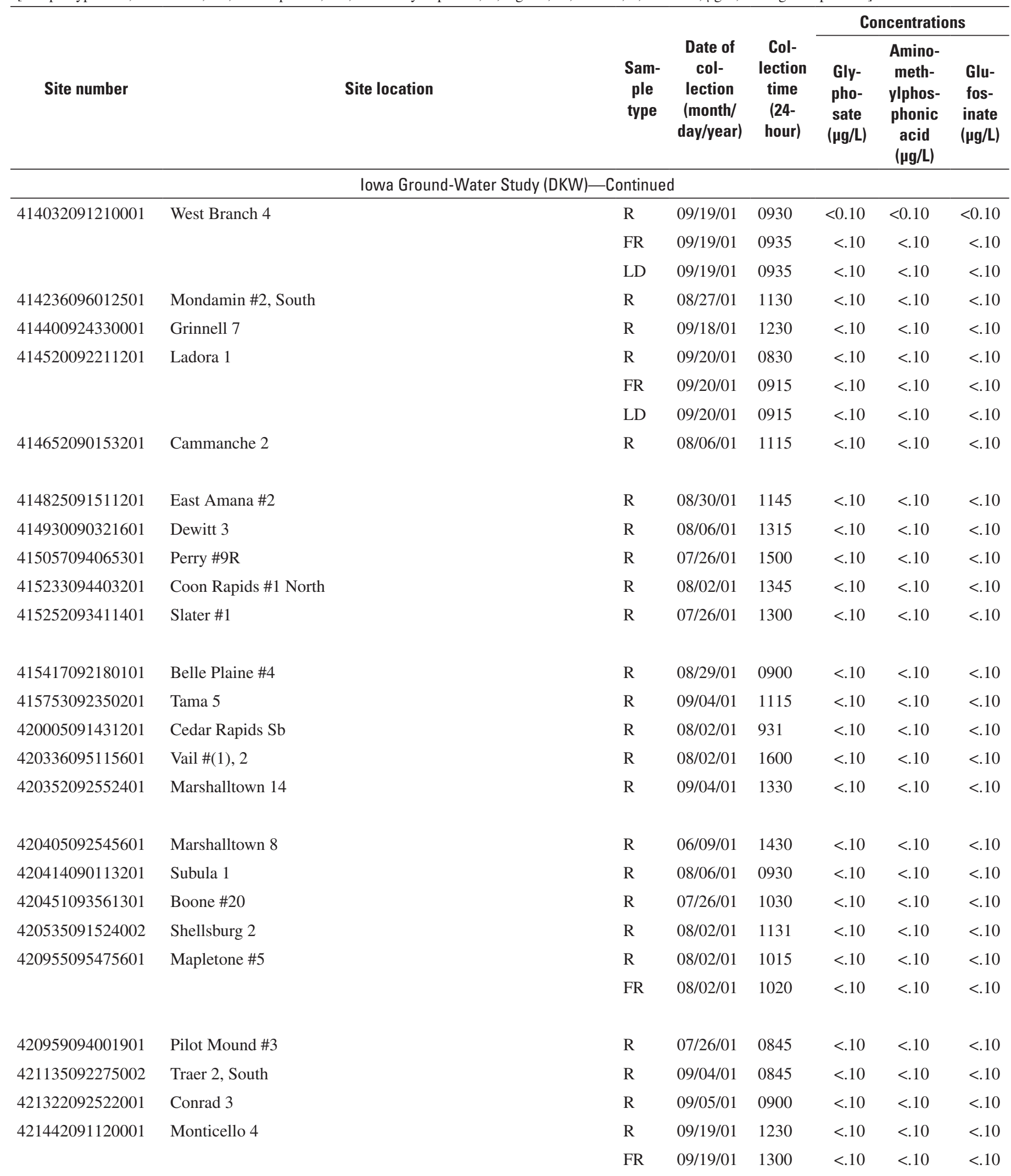


Table 3. Analytical results for glyphosate, aminomethylphosphonic acid, and glufosinate in State cooperative surface- and ground-water studies, 2001-06.-Continued

[Sample type: FB, field blank; FR, field replicate; LD, laboratory duplicate; R, regular; --, no data; <, less than; $\mu \mathrm{g} / \mathrm{L}$, micrograms per liter]

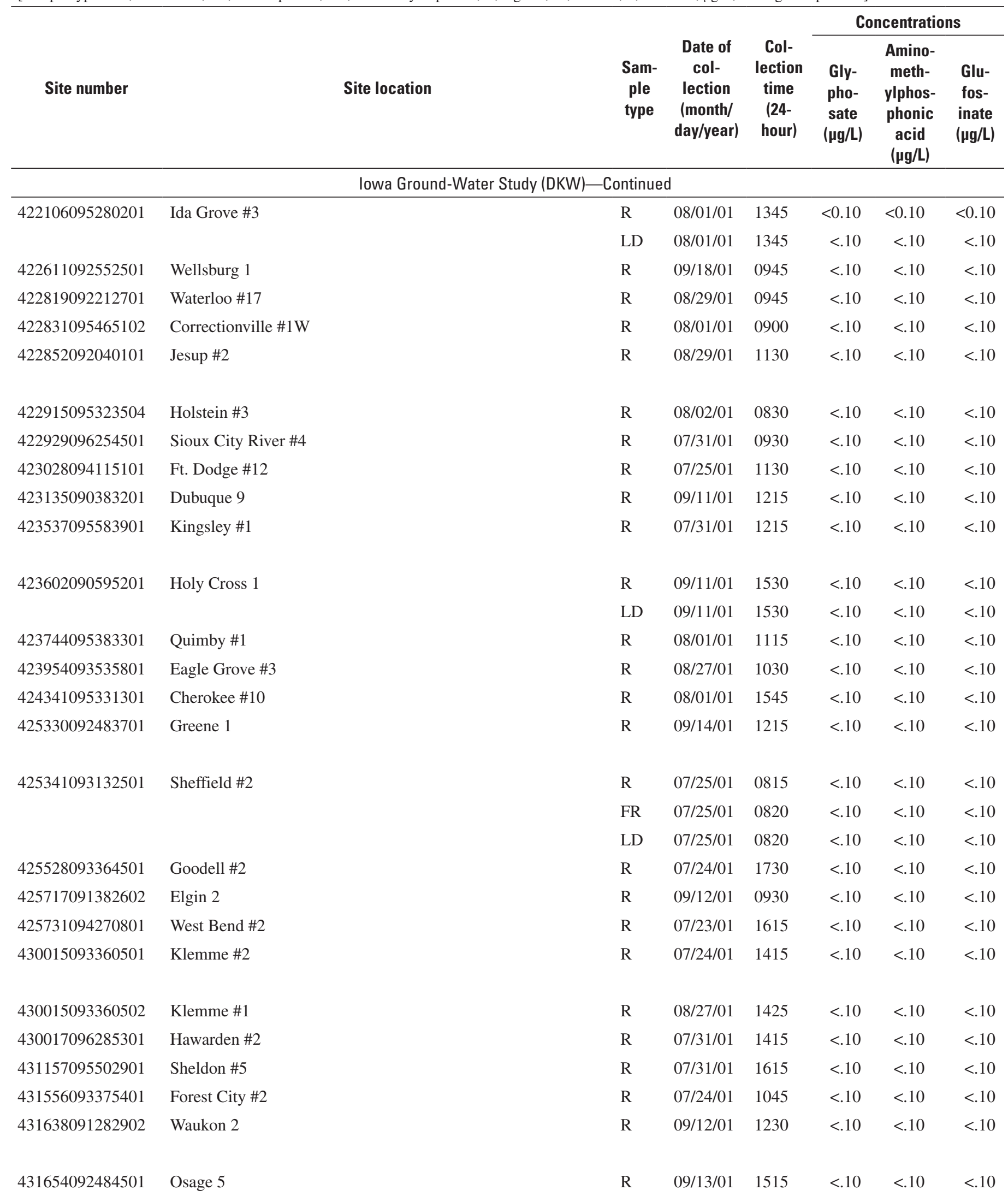


Table 3. Analytical results for glyphosate, aminomethylphosphonic acid, and glufosinate in State cooperative surface- and ground-water studies, 2001-06.-Continued

[Sample type: FB, field blank; FR, field replicate; LD, laboratory duplicate; R, regular; --, no data; <, less than; $\mu \mathrm{g} / \mathrm{L}$, micrograms per liter]

\begin{tabular}{|c|c|c|c|c|c|c|c|}
\hline \multirow[b]{2}{*}{ Site number } & \multirow[b]{2}{*}{ Site location } & \multirow[b]{2}{*}{$\begin{array}{c}\text { Sam- } \\
\text { ple } \\
\text { type }\end{array}$} & \multirow[b]{2}{*}{$\begin{array}{c}\text { Date of } \\
\text { col- } \\
\text { lection } \\
\text { (month/ } \\
\text { day/year) }\end{array}$} & \multirow[b]{2}{*}{$\begin{array}{c}\text { Col- } \\
\text { lection } \\
\text { time } \\
(24- \\
\text { hour) }\end{array}$} & \multicolumn{3}{|c|}{ Concentrations } \\
\hline & & & & & $\begin{array}{c}\text { Gly- } \\
\text { pho- } \\
\text { sate } \\
(\mu \mathrm{g} / \mathrm{L})\end{array}$ & $\begin{array}{c}\text { Amino- } \\
\text { meth- } \\
\text { ylphos- } \\
\text { phonic } \\
\text { acid } \\
(\mu \mathrm{g} / \mathrm{L})\end{array}$ & $\begin{array}{l}\text { Glu- } \\
\text { fos- } \\
\text { inate } \\
\text { ( } \mu \mathrm{g} / \mathrm{L})\end{array}$ \\
\hline \multicolumn{8}{|c|}{ lowa Ground-Water Study (DKW)—Continued } \\
\hline 431828091473201 & Decorah 6 & $\mathrm{R}$ & 09/12/01 & 1530 & $<0.10$ & $<0.10$ & $<0.10$ \\
\hline 432150092332401 & Riceville & $\mathrm{R}$ & 09/13/01 & 1215 & $<.10$ & $<.10$ & $<.10$ \\
\hline 432241092550802 & St. Ansgar 2 & $\mathrm{R}$ & $09 / 14 / 01$ & 0915 & $<.10$ & $<.10$ & $<.10$ \\
\hline 432349094285201 & Armstrong \#7 & $\mathrm{R}$ & $07 / 24 / 01$ & 0815 & $<.10$ & $<.10$ & $<.10$ \\
\hline 432608096201503 & Lester \#(4), 2 & $\mathrm{R}$ & $07 / 31 / 01$ & 1930 & $<.10$ & $<.10$ & $<.10$ \\
\hline 432650092170401 & Lime Springs & $\mathrm{R}$ & 09/13/01 & 0930 & $<.10$ & $<.10$ & $<.10$ \\
\hline \multicolumn{8}{|c|}{ Kansas Surface-Water Study (AZE) } \\
\hline \multirow[t]{6}{*}{07143672} & Little Arkansas River at Hwy 50 near Halstead & FB & $05 / 14 / 02$ & 0730 & $<.10$ & $<.10$ & $<.10$ \\
\hline & & $\mathrm{R}$ & $05 / 14 / 02$ & 1005 & .16 & .41 & $<.10$ \\
\hline & & $\mathrm{R}$ & $06 / 06 / 02$ & 1200 & $<.10$ & .26 & $<.10$ \\
\hline & & $\mathrm{R}$ & $06 / 17 / 02$ & 0915 & .75 & .50 & $<.10$ \\
\hline & & $\mathrm{R}$ & $04 / 22 / 03$ & 1010 & $<.10$ & .28 & $<.10$ \\
\hline & & $\mathrm{R}$ & $05 / 28 / 03$ & 1030 & .28 & .36 & $<.10$ \\
\hline \multirow[t]{5}{*}{07144100} & \multirow[t]{5}{*}{ Little Arkansas River near Sedgwick } & $\mathrm{R}$ & 05/13/02 & 1115 & .98 & .54 & $<.10$ \\
\hline & & $\mathrm{R}$ & 06/06/02 & 0900 & .66 & .41 & $<.10$ \\
\hline & & $\mathrm{R}$ & $06 / 13 / 02$ & 0930 & .43 & .49 & $<.10$ \\
\hline & & $\mathrm{R}$ & $04 / 23 / 03$ & 0950 & $<.10$ & .27 & $<.10$ \\
\hline & & $\mathrm{R}$ & $05 / 29 / 03$ & 1000 & .32 & .37 & $<.10$ \\
\hline \multicolumn{8}{|c|}{ Kansas Ground-Water Study (AZE) } \\
\hline 380643097353001 & Alta Mills well & $\mathrm{R}$ & $05 / 29 / 02$ & 1245 & $<.10$ & $<.10$ & $<.10$ \\
\hline \multirow[t]{2}{*}{380424097343801} & TH-10-95 & $\mathrm{R}$ & $05 / 28 / 02$ & 0945 & $<.10$ & $<.10$ & $<.10$ \\
\hline & & $\mathrm{R}$ & $05 / 29 / 02$ & 1020 & $<.10$ & $<.10$ & $<.10$ \\
\hline 380237097324401 & TH-02-95 & $\mathrm{R}$ & 05/29/02 & 1025 & $<.10$ & $<.10$ & $<.10$ \\
\hline 375304097291301 & TH-06-95 & $\mathrm{R}$ & $05 / 29 / 02$ & 1255 & $<.10$ & $<.10$ & $<.10$ \\
\hline 375628097270801 & TH-08-A3 & $\mathrm{R}$ & $05 / 30 / 02$ & 0910 & $<.10$ & $<.10$ & $<.10$ \\
\hline 375628097271701 & TH-08-A5 & $\mathrm{R}$ & 05/30/02 & 1110 & $<.10$ & $<.10$ & $<.10$ \\
\hline 375259097252901 & Sedgwick well & $\mathrm{R}$ & $05 / 31 / 02$ & 0925 & $<.10$ & $<.10$ & $<.10$ \\
\hline \multirow[t]{2}{*}{380028097311001} & EB-145-A1 & $\mathrm{R}$ & 06/03/02 & 0945 & $<.10$ & $<.10$ & $<.10$ \\
\hline & & FR & 06/03/02 & 1145 & $<.10$ & $<10$ & $<.10$ \\
\hline
\end{tabular}


Table 3. Analytical results for glyphosate, aminomethylphosphonic acid, and glufosinate in State cooperative surface- and ground-water studies, 2001-06.-Continued

[Sample type: FB, field blank; FR, field replicate; LD, laboratory duplicate; R, regular; --, no data; <, less than; $\mu \mathrm{g} / \mathrm{L}$, micrograms per liter]

\begin{tabular}{|c|c|c|c|c|c|c|c|}
\hline \multirow[b]{2}{*}{ Site number } & & \multirow[b]{2}{*}{ Site location } & \multirow[b]{2}{*}{$\begin{array}{c}\text { Date of } \\
\text { col- } \\
\text { lection } \\
\text { (month/ } \\
\text { day/year) }\end{array}$} & \multirow[b]{2}{*}{$\begin{array}{l}\text { Col- } \\
\text { lection } \\
\text { time } \\
(24- \\
\text { hour) }\end{array}$} & \multicolumn{3}{|c|}{ Concentrations } \\
\hline & & & & & $\begin{array}{l}\text { Gly- } \\
\text { pho- } \\
\text { sate } \\
\text { ( } \mu \mathrm{g} / \mathrm{L})\end{array}$ & $\begin{array}{c}\text { Amino- } \\
\text { meth- } \\
\text { ylphos- } \\
\text { phonic } \\
\text { acid } \\
\text { ( } \mathrm{rg} / \mathrm{L})\end{array}$ & $\begin{array}{c}\text { Glu- } \\
\text { fos- } \\
\text { inate } \\
\text { ( } \mu \mathrm{g} / \mathrm{L} \text { ) }\end{array}$ \\
\hline \multicolumn{8}{|c|}{ Kansas Ground-Water Study (AZE) —Continued } \\
\hline 380143097344201 & IW-06A & $\mathrm{R}$ & $06 / 24 / 02$ & 1005 & $<0.10$ & 0.33 & $<0.10$ \\
\hline 380143097344202 & IW-06C & $\mathrm{R}$ & $06 / 24 / 02$ & 1225 & $<.10$ & $<.10$ & $<.10$ \\
\hline 380016097384901 & IW-08A & $\mathrm{R}$ & $06 / 25 / 02$ & 0950 & $<.10$ & $<.10$ & $<.10$ \\
\hline 380016097384902 & IW-08C & $\mathrm{R}$ & $06 / 25 / 02$ & 1245 & $<.10$ & $<.10$ & $<.10$ \\
\hline 380328097342501 & IW-03A & $\mathrm{R}$ & 06/26/02 & 0950 & $<.10$ & $<.10$ & $<.10$ \\
\hline 380328097342502 & IW-03C (deep) & $\mathrm{R}$ & 06/26/02 & 1150 & $<.10$ & $<.10$ & $<.10$ \\
\hline \multirow[t]{2}{*}{380421097385001} & IW-01A & $\mathrm{R}$ & 06/27/02 & 0945 & $<.10$ & $<.10$ & $<.10$ \\
\hline & & LD & 06/27/02 & 0945 & $<.10$ & $<.10$ & $<.10$ \\
\hline \multirow[t]{2}{*}{380421097385002} & IW-01C & $\mathrm{R}$ & $06 / 27 / 02$ & 1205 & $<.10$ & $<.10$ & $<.10$ \\
\hline & & LD & 06/27/02 & 1205 & $<.10$ & $<.10$ & $<.10$ \\
\hline 375958097300001 & IW-12A & $\mathrm{R}$ & 07/08/02 & 0940 & $<.10$ & $<.10$ & $<.10$ \\
\hline 375958097300002 & IW-12C & $\mathrm{R}$ & 07/08/02 & 1200 & $<.10$ & $<.10$ & $<.10$ \\
\hline 375630097342701 & IW-20A & $\mathrm{R}$ & 07/10/02 & 0905 & $<.10$ & $<.10$ & $<.10$ \\
\hline 375630097342702 & IW-20C & $\mathrm{R}$ & 07/10/02 & 1150 & $<.10$ & $<.10$ & $<.10$ \\
\hline 375629097293701 & IW-22A & $\mathrm{R}$ & $07 / 11 / 02$ & 0930 & $<.10$ & $<.10$ & $<.10$ \\
\hline 375629097293702 & IW-22C & $\mathrm{R}$ & $07 / 11 / 02$ & 1200 & $<.10$ & $<.10$ & $<.10$ \\
\hline 375446097390701 & IW-24A & $\mathrm{R}$ & 07/16/02 & 0950 & $<.10$ & $<.10$ & $<.10$ \\
\hline \multirow[t]{2}{*}{375446097390702} & IW-24C & $\mathrm{R}$ & 07/16/02 & 1140 & $<.10$ & $<.10$ & $<.10$ \\
\hline & & LD & 07/16/02 & 1140 & $<.10$ & $<.10$ & $<.10$ \\
\hline 375258097340601 & IW-30A & $\mathrm{R}$ & 07/18/02 & 0850 & $<.10$ & $<.10$ & $<.10$ \\
\hline 375258097340602 & IW-30C & $\mathrm{R}$ & 07/18/02 & 1105 & $<.10$ & $<.10$ & $<.10$ \\
\hline 375116097274701 & IW-37A & $\mathrm{R}$ & $07 / 25 / 02$ & 0900 & $<.10$ & $<.10$ & $<.10$ \\
\hline 375116097274702 & IW-37C & $\mathrm{R}$ & $07 / 25 / 02$ & 1130 & $<.10$ & $<.10$ & $<.10$ \\
\hline 380356097340901 & DW-TW-06 & $\mathrm{R}$ & $12 / 03 / 02$ & 1305 & $<.10$ & $<.10$ & $<.10$ \\
\hline 380358097341301 & DW-TW-07 & $\mathrm{R}$ & $12 / 03 / 02$ & 1040 & $<.10$ & $<.10$ & $<.10$ \\
\hline 380142097363601 & RRW-03 & $\mathrm{R}$ & $12 / 09 / 02$ & 1050 & $<.10$ & $<.10$ & $<.10$ \\
\hline \multirow[t]{2}{*}{380329097363703} & RRW-01 & $\mathrm{R}$ & $12 / 10 / 02$ & 1300 & $<.10$ & $<.10$ & $<.10$ \\
\hline & & LD & $12 / 10 / 02$ & 1300 & $<.10$ & $<.10$ & $<.10$ \\
\hline 380336097335801 & DW-TW-03 & $\mathrm{R}$ & $12 / 10 / 02$ & 1015 & $<.10$ & $<.10$ & $<.10$ \\
\hline
\end{tabular}


Table 3. Analytical results for glyphosate, aminomethylphosphonic acid, and glufosinate in State cooperative surface- and ground-water studies, 2001-06.-Continued

[Sample type: FB, field blank; FR, field replicate; LD, laboratory duplicate; R, regular; --, no data; <, less than; $\mu \mathrm{g} / \mathrm{L}$, micrograms per liter]

\begin{tabular}{|c|c|c|c|c|c|c|c|}
\hline \multirow[b]{2}{*}{ Site number } & & \multirow[b]{2}{*}{$\begin{array}{c}\text { Sam- } \\
\text { ple } \\
\text { type }\end{array}$} & \multirow[b]{2}{*}{$\begin{array}{c}\text { Date of } \\
\text { col- } \\
\text { lection } \\
\text { (month/ } \\
\text { day/year) }\end{array}$} & \multirow[b]{2}{*}{$\begin{array}{c}\text { Col- } \\
\text { lection } \\
\text { time } \\
\text { (24- } \\
\text { hour) }\end{array}$} & \multicolumn{3}{|c|}{ Concentrations } \\
\hline & & & & & $\begin{array}{l}\text { Gly- } \\
\text { pho- } \\
\text { sate } \\
(\mu \mathrm{g} / \mathrm{L})\end{array}$ & $\begin{array}{c}\text { Amino- } \\
\text { meth- } \\
\text { ylphos- } \\
\text { phonic } \\
\text { acid } \\
(\mu \mathrm{g} / \mathrm{L}) \\
\end{array}$ & $\begin{array}{l}\text { Glu- } \\
\text { fos- } \\
\text { inate } \\
(\mu \mathrm{g} / \mathrm{L})\end{array}$ \\
\hline \multicolumn{8}{|c|}{ Kansas Ground-Water Study (AZE)—Continued } \\
\hline 380338097340201 & DW-TW-04 & $\mathrm{R}$ & $12 / 11 / 02$ & 1015 & $<0.10$ & $<0.10$ & $<0.10$ \\
\hline 380344097340401 & DW-TW-05 & $\mathrm{R}$ & $12 / 11 / 02$ & 1215 & $<.10$ & $<.10$ & $<.10$ \\
\hline 380329097334601 & DW-TW-01 & $\mathrm{R}$ & $12 / 12 / 02$ & 1030 & $<.10$ & $<.10$ & $<.10$ \\
\hline 380333097335001 & DW-TW-02 & $\mathrm{R}$ & $12 / 12 / 02$ & 1015 & $<.10$ & $<.10$ & $<.10$ \\
\hline 380235097363801 & RRW-02 & $\mathrm{R}$ & 03/06/03 & 1225 & $<.10$ & $<.10$ & $<.10$ \\
\hline \multicolumn{8}{|c|}{ Kansas Surface-Water Study (JUR) } \\
\hline \multirow[t]{2}{*}{--} & Bronson City Lake & $\mathrm{R}$ & $04 / 03 / 03$ & 1300 & $<.10$ & $<.10$ & $<.10$ \\
\hline & & $\mathrm{LD}$ & $04 / 03 / 03$ & 1300 & $<.10$ & $<.10$ & $<.10$ \\
\hline \multirow[t]{2}{*}{--} & Centralia Lake, Centralia & $\mathrm{R}$ & $05 / 02 / 03$ & 1000 & $<.10$ & $<.10$ & $<.10$ \\
\hline & & $\mathrm{LD}$ & 05/02/03 & 1000 & $<.10$ & .10 & $<.10$ \\
\hline-- & Crystal Lake, Garnett & $\mathrm{R}$ & $04 / 10 / 03$ & 1030 & $<.10$ & $<.10$ & $<.10$ \\
\hline-- & Edgerton City Lake & $\mathrm{R}$ & $11 / 12 / 02$ & 1000 & $<.10$ & .19 & $<.10$ \\
\hline-- & Gardner City Lake & $\mathrm{R}$ & $10 / 21 / 02$ & 1100 & $<.10$ & $<.10$ & $<.10$ \\
\hline-- & Hiawatha City Lake & $\mathrm{R}$ & $10 / 02 / 02$ & 1200 & $<.10$ & .19 & $<.10$ \\
\hline-- & Lake Afton & $\mathrm{R}$ & $10 / 17 / 02$ & 1230 & $<.10$ & $<.10$ & $<.10$ \\
\hline-- & Mission Lake & $\mathrm{R}$ & $10 / 23 / 02$ & 1000 & $<.10$ & .27 & $<.10$ \\
\hline-- & Mission Lake & $\mathrm{R}$ & $05 / 21 / 03$ & 1300 & $<.10$ & .31 & $<.10$ \\
\hline-- & Pony Creek Lake, Sabetha & $\mathrm{R}$ & $05 / 21 / 03$ & 1000 & $<.10$ & $<.10$ & $<.10$ \\
\hline & & & & & & & \\
\hline \multirow[t]{2}{*}{--} & 9F-D Manhattan & $\mathrm{R}$ & $10 / 18 / 01$ & -- & $<.02$ & $<.02$ & $<.02$ \\
\hline & & FR & $10 / 18 / 01$ & -- & $<.02$ & $<.02$ & $<.02$ \\
\hline-- & $9 \mathrm{~F}-\mathrm{G}$ & FR & $10 / 18 / 01$ & -- & $<.02$ & $<.02$ & $<.02$ \\
\hline-- & Fairway \#1 & $\mathrm{R}$ & $05 / 21 / 03$ & 1000 & 3.3 & 6.1 & $<.02$ \\
\hline-- & Fairway \#2 & $\mathrm{R}$ & 05/07/02 & 1200 & 5.2 & 22 & $<.02$ \\
\hline \multirow[t]{2}{*}{--} & Fairway \#3 & $\mathrm{R}$ & 07/02/03 & 0845 & .63 & .80 & $<.02$ \\
\hline & & $\mathrm{R}$ & 07/23/03 & 0830 & $<.02$ & .64 & $<.02$ \\
\hline \multirow[t]{2}{*}{--} & Fairway Drain \#1 & $\mathrm{R}$ & 05/07/02 & 1200 & 1.2 & 5.4 & $<.02$ \\
\hline & & $\mathrm{R}$ & $05 / 12 / 03$ & 0900 & .33 & 1.0 & $<.02$ \\
\hline \multirow[t]{2}{*}{--} & Fairway Drain \#2 & $\mathrm{R}$ & $05 / 12 / 03$ & 0900 & .59 & 1.6 & $<.02$ \\
\hline & & $\mathrm{R}$ & 06/20/02 & 0930 & .42 & 1.2 & $<.02$ \\
\hline
\end{tabular}


Table 3. Analytical results for glyphosate, aminomethylphosphonic acid, and glufosinate in State cooperative surface- and ground-water studies, 2001-06.-Continued

[Sample type: FB, field blank; FR, field replicate; LD, laboratory duplicate; R, regular; --, no data; <, less than; $\mu \mathrm{g} / \mathrm{L}$, micrograms per liter]

\begin{tabular}{|c|c|c|c|c|c|c|c|}
\hline \multirow[b]{2}{*}{ Site number } & \multirow[b]{2}{*}{ Site location } & \multirow[b]{2}{*}{$\begin{array}{c}\text { Sam- } \\
\text { ple } \\
\text { type }\end{array}$} & \multirow[b]{2}{*}{$\begin{array}{c}\text { Date of } \\
\text { col- } \\
\text { lection } \\
\text { (month/ } \\
\text { day/year) }\end{array}$} & \multirow[b]{2}{*}{$\begin{array}{c}\text { Col- } \\
\text { lection } \\
\text { time } \\
\text { (24- } \\
\text { hour) }\end{array}$} & \multicolumn{3}{|c|}{ Concentrations } \\
\hline & & & & & $\begin{array}{l}\text { Gly- } \\
\text { pho- } \\
\text { sate } \\
\text { ( } \mu \mathrm{g} / \mathrm{L})\end{array}$ & $\begin{array}{l}\text { Amino- } \\
\text { meth- } \\
\text { ylphos- } \\
\text { phonic } \\
\text { acid } \\
\text { ( } \mathrm{gg} / \mathrm{L} \text { ) }\end{array}$ & $\begin{array}{l}\text { Glu- } \\
\text { fos- } \\
\text { inate } \\
\text { ( } \mu \mathrm{g} / \mathrm{L} \text { ) }\end{array}$ \\
\hline \multicolumn{8}{|c|}{ Kansas Surface-Water Study (KSU)_Continued } \\
\hline \multirow[t]{2}{*}{--} & Fairway Drain \#3 & $\mathrm{R}$ & 05/07/02 & 1200 & 0.47 & 1.2 & $<0.02$ \\
\hline & & $\mathrm{R}$ & $05 / 12 / 03$ & 0900 & 1.0 & 2.5 & $<.02$ \\
\hline \multirow[t]{2}{*}{--} & Inlet \#1 & $\mathrm{R}$ & 05/05/02 & 1932 & $<.02$ & $<.02$ & $<.02$ \\
\hline & & $\mathrm{R}$ & 05/10/02 & 2200 & $<.02$ & $<.02$ & $<.02$ \\
\hline \multirow[t]{3}{*}{--} & Inlet \#4 & $\mathrm{R}$ & $04 / 23 / 03$ & 1504 & $<.02$ & $<.02$ & $<.02$ \\
\hline & & LD & $04 / 23 / 03$ & 1504 & $<.02$ & $<.02$ & $<.02$ \\
\hline & & $\mathrm{R}$ & $04 / 29 / 02$ & 0306 & .16 & $<.02$ & $<.02$ \\
\hline \multirow[t]{2}{*}{--} & Outlet \#5 & $\mathrm{R}$ & $05 / 24 / 03$ & 0643 & $<.02$ & $<.02$ & $<.02$ \\
\hline & & $\mathrm{R}$ & $05 / 24 / 03$ & 0726 & $<.02$ & .26 & $<.02$ \\
\hline-- & Pond \#1, Site \#1, 0.75 foot & $\mathrm{R}$ & $04 / 16 / 03$ & -- & $<.02$ & $<.02$ & $<.02$ \\
\hline-- & Pond \#2, Site \#1, 1.0 foot & $\mathrm{R}$ & $04 / 16 / 03$ & -- & $<.02$ & $<.02$ & $<.02$ \\
\hline-- & Pond 2, Sample 4, 6 foot & $\mathrm{R}$ & 08/06/03 & -- & $<.02$ & $<.02$ & $<.02$ \\
\hline-- & Pond 2, Sample 5, 8 foot & $\mathrm{R}$ & 08/06/03 & -- & $<.02$ & $<.02$ & $<.02$ \\
\hline \multicolumn{8}{|c|}{ Mississippi Surface-Water Study (RRM) } \\
\hline-- & 1617-TL3 & $\mathrm{R}$ & 08/27/01 & 2114 & $<.10$ & $<.10$ & $<.10$ \\
\hline-- & 1610-BL3 & $\mathrm{R}$ & $08 / 31 / 01$ & 0626 & 1.0 & 2.5 & $<.10$ \\
\hline-- & 1618-TL3 & $\mathrm{R}$ & 08/31/01 & 0729 & $<.10$ & $<.10$ & $<.10$ \\
\hline-- & 1622-UL1 & $\mathrm{R}$ & 08/31/01 & 0800 & .23 & .81 & $<.10$ \\
\hline-- & 1619-TL3 & $\mathrm{R}$ & 09/01/01 & 0806 & $<.10$ & $<.10$ & $<.10$ \\
\hline-- & 1614-BL4B & $\mathrm{R}$ & $08 / 31 / 01$ & 0837 & .52 & 1.1 & $<.10$ \\
\hline-- & 1609-BL4 & $\mathrm{R}$ & $08 / 31 / 01$ & 0912 & .68 & 2.5 & $<.10$ \\
\hline-- & 1611-BL3 & $\mathrm{R}$ & 09/01/01 & 0823 & .88 & 2.9 & $<.10$ \\
\hline-- & 1623-UL1 & $\mathrm{R}$ & 09/01/01 & 0920 & .22 & .74 & $<.10$ \\
\hline-- & 1615-UL2 & $\mathrm{R}$ & 09/01/01 & 0945 & .25 & 1.7 & $<.10$ \\
\hline-- & 1616-UL2 & $\mathrm{R}$ & 09/02/01 & 0820 & .32 & 1.2 & $<.10$ \\
\hline-- & 1616-TL2 & $\mathrm{R}$ & 09/02/01 & 1305 & .58 & .68 & $<.10$ \\
\hline \multirow[t]{2}{*}{--} & 1615-BL4B & $\mathrm{R}$ & 09/02/01 & 1412 & .31 & 1.4 & $<.10$ \\
\hline & & $\mathrm{LD}$ & 09/02/01 & 1412 & .20 & 1.0 & $<.10$ \\
\hline-- & 2601-UL1 & $\mathrm{R}$ & $10 / 11 / 01$ & 1437 & .43 & .77 & $<.10$ \\
\hline
\end{tabular}


Table 3. Analytical results for glyphosate, aminomethylphosphonic acid, and glufosinate in State cooperative surface- and ground-water studies, 2001-06. - Continued

[Sample type: FB, field blank; FR, field replicate; LD, laboratory duplicate; R, regular; --, no data; <, less than; $\mu \mathrm{g} / \mathrm{L}$, micrograms per liter]

\begin{tabular}{|c|c|c|c|c|c|c|c|}
\hline \multirow[b]{2}{*}{ Site number } & & \multirow[b]{2}{*}{$\begin{array}{c}\text { Sam- } \\
\text { ple } \\
\text { type }\end{array}$} & \multirow[b]{2}{*}{$\begin{array}{c}\text { Date of } \\
\text { col- } \\
\text { lection } \\
\text { (month/ } \\
\text { day/year) }\end{array}$} & \multirow[b]{2}{*}{$\begin{array}{c}\text { Col- } \\
\text { lection } \\
\text { time } \\
\text { (24- } \\
\text { hour) }\end{array}$} & \multicolumn{3}{|c|}{ Concentrations } \\
\hline & & & & & $\begin{array}{c}\text { Gly- } \\
\text { pho- } \\
\text { sate } \\
\text { ( } \mu \mathrm{g} / \mathrm{L})\end{array}$ & $\begin{array}{l}\text { Amino- } \\
\text { meth- } \\
\text { ylphos- } \\
\text { phonic } \\
\text { acid } \\
\text { (pg/L) }\end{array}$ & $\begin{array}{c}\text { Glu- } \\
\text { fos- } \\
\text { inate } \\
(\mu \mathrm{g} / \mathrm{L})\end{array}$ \\
\hline \multicolumn{8}{|c|}{ Mississippi Surface-Water Study (RRM)_Continued } \\
\hline-- & 2601-UL2 & $\mathrm{R}$ & 10/11/01 & 1537 & 0.23 & 1.0 & $<0.10$ \\
\hline-- & 2601-TL2 & $\mathrm{R}$ & $10 / 12 / 01$ & 1312 & .20 & .33 & $<.10$ \\
\hline-- & 2602-UL1 & $\mathrm{R}$ & 10/13/01 & 0850 & .40 & 1.1 & $<.10$ \\
\hline-- & 2602-UL2 & $\mathrm{R}$ & $10 / 13 / 01$ & 0913 & .22 & .85 & $<.10$ \\
\hline-- & 2601-BL4A & $\mathrm{R}$ & $10 / 13 / 01$ & 0945 & 1.3 & 2.4 & $<.10$ \\
\hline-- & 2601-BL3 & $\mathrm{R}$ & $11 / 26 / 01$ & 1855 & .88 & 2.0 & $<.10$ \\
\hline-- & 2603-UL2 & $\mathrm{R}$ & $11 / 27 / 01$ & 0827 & .49 & 1.6 & $<.10$ \\
\hline-- & 2602-TL2 & $\mathrm{R}$ & $11 / 27 / 01$ & 0215 & .25 & .49 & $<.10$ \\
\hline-- & 2601-TL3 & $\mathrm{R}$ & $11 / 27 / 01$ & 0255 & .60 & $<.10$ & $<.10$ \\
\hline-- & 2602-BL4A & $\mathrm{R}$ & $11 / 27 / 01$ & 0258 & .40 & 1.7 & $<.10$ \\
\hline-- & 2603-UL1 & $\mathrm{R}$ & $11 / 27 / 01$ & 0300 & .29 & .86 & $<.10$ \\
\hline-- & 2604-UL2 & $\mathrm{R}$ & $11 / 28 / 01$ & 1111 & .42 & .86 & $<.10$ \\
\hline-- & 2604-UL1 & $\mathrm{R}$ & $11 / 28 / 01$ & 1118 & .52 & .76 & $<.10$ \\
\hline-- & 2603-TL2 & $\mathrm{R}$ & $11 / 28 / 01$ & 1341 & .43 & .33 & $<.10$ \\
\hline-- & 2602-BL3 & $\mathrm{R}$ & $11 / 28 / 01$ & 1402 & .49 & 1.5 & $<.10$ \\
\hline-- & 2602-TL3 & $\mathrm{R}$ & $11 / 28 / 01$ & 1812 & .41 & $<.10$ & $<.10$ \\
\hline-- & 2604-BL4A & $\mathrm{R}$ & $11 / 28 / 01$ & 1815 & .27 & .63 & $<.10$ \\
\hline \multirow[t]{2}{*}{--} & 2605-BL4A & $\mathrm{R}$ & $12 / 12 / 01$ & 1251 & .34 & .70 & $<.10$ \\
\hline & & LD & $12 / 12 / 01$ & 1251 & .29 & .55 & $<.10$ \\
\hline-- & 2603-TL3 & $\mathrm{R}$ & $12 / 12 / 01$ & 1314 & $<.10$ & $<.10$ & $<.10$ \\
\hline-- & 2604-TL2 & $\mathrm{R}$ & $12 / 12 / 01$ & 1316 & .43 & .24 & $<.10$ \\
\hline-- & 2605-UL1 & $\mathrm{R}$ & $12 / 12 / 01$ & 1319 & 2.0 & .66 & $<.10$ \\
\hline-- & 2605-UL2 & $\mathrm{R}$ & $12 / 12 / 01$ & 1353 & .14 & .66 & $<.10$ \\
\hline-- & 2606-UL2 & $\mathrm{R}$ & $12 / 12 / 01$ & 2318 & .11 & .89 & $<.10$ \\
\hline-- & 2601-BL1 & $\mathrm{R}$ & $12 / 13 / 01$ & 1558 & .48 & 1.1 & $<.10$ \\
\hline-- & 2606-BL4A & $\mathrm{R}$ & $12 / 15 / 01$ & 1452 & .14 & .36 & $<.10$ \\
\hline-- & 2604-TL3 & $\mathrm{R}$ & $12 / 17 / 01$ & 0311 & .13 & $<.10$ & $<.10$ \\
\hline-- & 2607-UL2 & $\mathrm{R}$ & $12 / 17 / 01$ & 0347 & .17 & .75 & $<.10$ \\
\hline-- & 2606-UL1 & $\mathrm{R}$ & $01 / 22 / 02$ & 1845 & $<.10$ & .47 & $<.10$ \\
\hline-- & 2601-BL4 & $\mathrm{R}$ & 01/23/02 & 0138 & .33 & .70 & $<.10$ \\
\hline
\end{tabular}


Table 3. Analytical results for glyphosate, aminomethylphosphonic acid, and glufosinate in State cooperative surface- and ground-water studies, 2001-06.-Continued

[Sample type: FB, field blank; FR, field replicate; LD, laboratory duplicate; R, regular; --, no data; <, less than; $\mu \mathrm{g} / \mathrm{L} ;$ micrograms per liter]

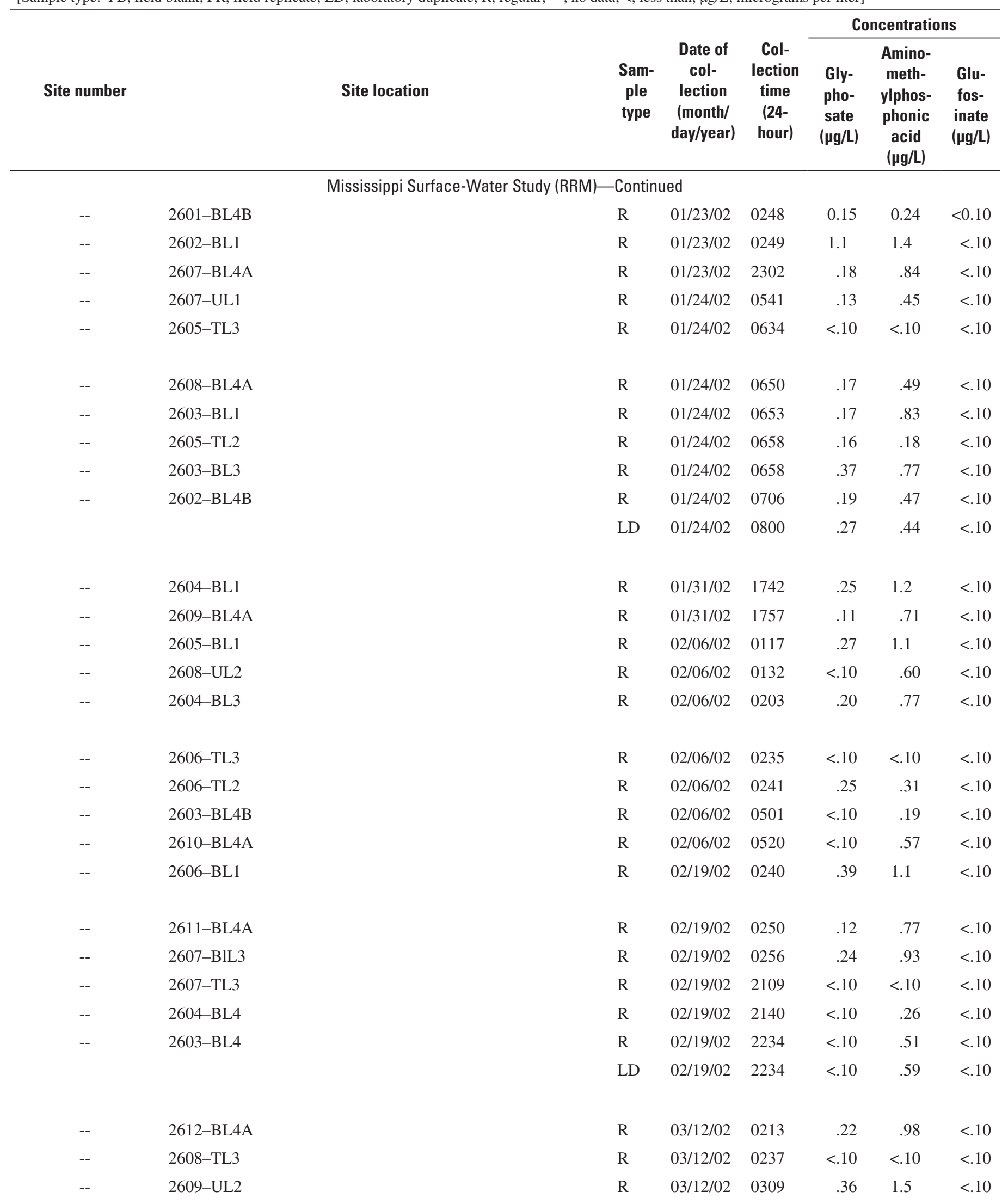


Table 3. Analytical results for glyphosate, aminomethylphosphonic acid, and glufosinate in State cooperative surface- and ground-water studies, 2001-06.-Continued

[Sample type: FB, field blank; FR, field replicate; LD, laboratory duplicate; R, regular; --, no data; <, less than; $\mu \mathrm{g} / \mathrm{L}$; micrograms per liter]

\begin{tabular}{|c|c|c|c|c|c|c|c|}
\hline \multirow[b]{2}{*}{ Site number } & & \multirow[b]{2}{*}{$\begin{array}{c}\text { Sam- } \\
\text { ple } \\
\text { type }\end{array}$} & \multirow[b]{2}{*}{$\begin{array}{c}\text { Date of } \\
\text { col- } \\
\text { lection } \\
\text { (month/ } \\
\text { day/year) }\end{array}$} & \multirow[b]{2}{*}{$\begin{array}{c}\text { Col- } \\
\text { lection } \\
\text { time } \\
\text { (24- } \\
\text { hour) }\end{array}$} & \multicolumn{3}{|c|}{ Concentrations } \\
\hline & & & & & $\begin{array}{l}\text { Gly- } \\
\text { pho- } \\
\text { sate } \\
(\mu \mathrm{g} / \mathrm{L})\end{array}$ & $\begin{array}{c}\text { Amino- } \\
\text { meth- } \\
\text { ylphos- } \\
\text { phonic } \\
\text { acid } \\
\text { ( } \mathrm{gg} / \mathrm{L})\end{array}$ & $\begin{array}{c}\text { Glu- } \\
\text { fos- } \\
\text { inate } \\
\text { ( } \mu \mathrm{g} / \mathrm{L})\end{array}$ \\
\hline \multicolumn{8}{|c|}{ Mississippi Surface-Water Study (RRM)_Continued } \\
\hline \multirow[t]{2}{*}{--} & 2605-BL4B & $\mathrm{R}$ & $03 / 12 / 02$ & 0350 & 0.49 & 0.44 & $<0.10$ \\
\hline & & LD & $03 / 12 / 02$ & 0350 & .43 & .33 & $<.10$ \\
\hline-- & 2604-BL4 & $\mathrm{R}$ & $03 / 12 / 02$ & 0500 & .21 & .90 & $<.10$ \\
\hline-- & 2606-BL4B & $\mathrm{R}$ & $03 / 16 / 02$ & 0100 & 2.7 & 1.2 & $<.10$ \\
\hline-- & 2613-BL4A & $\mathrm{R}$ & $03 / 16 / 02$ & 0122 & 5.7 & 2.3 & $<.10$ \\
\hline-- & 2609-TL3 & $\mathrm{R}$ & $03 / 16 / 02$ & 0128 & .28 & .22 & $<0.10$ \\
\hline-- & 2608-UL1 & $\mathrm{R}$ & $03 / 16 / 02$ & 0147 & .20 & .62 & $<.10$ \\
\hline-- & 2609-TL2 & $\mathrm{R}$ & $03 / 16 / 02$ & 0155 & .53 & .72 & $<.10$ \\
\hline-- & 2610-UL2 & $\mathrm{R}$ & $03 / 16 / 02$ & 0158 & .27 & 1.3 & $<.10$ \\
\hline-- & 2605-BL4 & $\mathrm{R}$ & $03 / 16 / 02$ & 0309 & 9.8 & 2.5 & $<.10$ \\
\hline \multirow[t]{2}{*}{--} & 2607-BL1 & $\mathrm{R}$ & $03 / 20 / 02$ & 0515 & .48 & 2.0 & $<.10$ \\
\hline & & LD & $03 / 20 / 02$ & 0515 & .44 & 2.2 & $<.10$ \\
\hline-- & 2609-UL1 & $\mathrm{R}$ & 03/20/02 & 0526 & .25 & .76 & $<.10$ \\
\hline-- & 2611-UL2 & $\mathrm{R}$ & $03 / 20 / 02$ & 0545 & .36 & 1.4 & $<.10$ \\
\hline-- & 2610-TL3 & $\mathrm{R}$ & 03/20/02 & 1148 & .43 & $<.10$ & $<.10$ \\
\hline-- & 2614-BL4A & $\mathrm{R}$ & $03 / 20 / 02$ & 1155 & 1.1 & 1.5 & $<.10$ \\
\hline-- & 2607-BL4B & $\mathrm{R}$ & $03 / 20 / 02$ & 1155 & .36 & 1.6 & $<.10$ \\
\hline-- & 2606-BL4 & $\mathrm{R}$ & $03 / 20 / 02$ & 1304 & 1.1 & 1.4 & $<.10$ \\
\hline-- & 2608-BL1 & $\mathrm{R}$ & 03/30/02 & 2136 & .47 & 1.9 & $<.10$ \\
\hline-- & 2611-TL3 & $\mathrm{R}$ & 03/30/02 & 2139 & $<.10$ & $<.10$ & $<.10$ \\
\hline-- & 2611-TL3 & LD & $03 / 30 / 02$ & 2139 & .10 & $<.10$ & $<.10$ \\
\hline-- & 2615-BL4A & $\mathrm{R}$ & 03/30/02 & 2156 & .51 & 1.1 & $<.10$ \\
\hline-- & 2612-UL2 & $\mathrm{R}$ & 03/30/02 & 2309 & .11 & 1.2 & $<.10$ \\
\hline-- & 2611-TL2 & $\mathrm{R}$ & 03/30/02 & 2310 & 2.1 & .67 & $<.10$ \\
\hline-- & 2608-BL4B & $\mathrm{R}$ & $03 / 31 / 02$ & 0150 & .38 & 2.2 & $<.10$ \\
\hline-- & 2607-BL4 & $\mathrm{R}$ & $03 / 31 / 02$ & 0320 & .59 & .65 & $<.10$ \\
\hline-- & 2607-BL4 & $\mathrm{LD}$ & $03 / 31 / 02$ & 0320 & .49 & .54 & $<.10$ \\
\hline-- & 2612-TL3 & $\mathrm{R}$ & $04 / 08 / 02$ & 1107 & $<.10$ & $<.10$ & $<.10$ \\
\hline-- & 2609-BL1 & $\mathrm{R}$ & 04/08/02 & 1110 & 99 & 3.4 & $<.10$ \\
\hline-- & 2616-BL4A & $\mathrm{R}$ & 04/08/02 & 1143 & .80 & .58 & $<.10$ \\
\hline
\end{tabular}


Table 3. Analytical results for glyphosate, aminomethylphosphonic acid, and glufosinate in State cooperative surface- and ground-water studies, 2001-06.-Continued

[Sample type: FB, field blank; FR, field replicate; LD, laboratory duplicate; R, regular; --, no data; <, less than; $\mu \mathrm{g} / \mathrm{L}$, micrograms per liter]

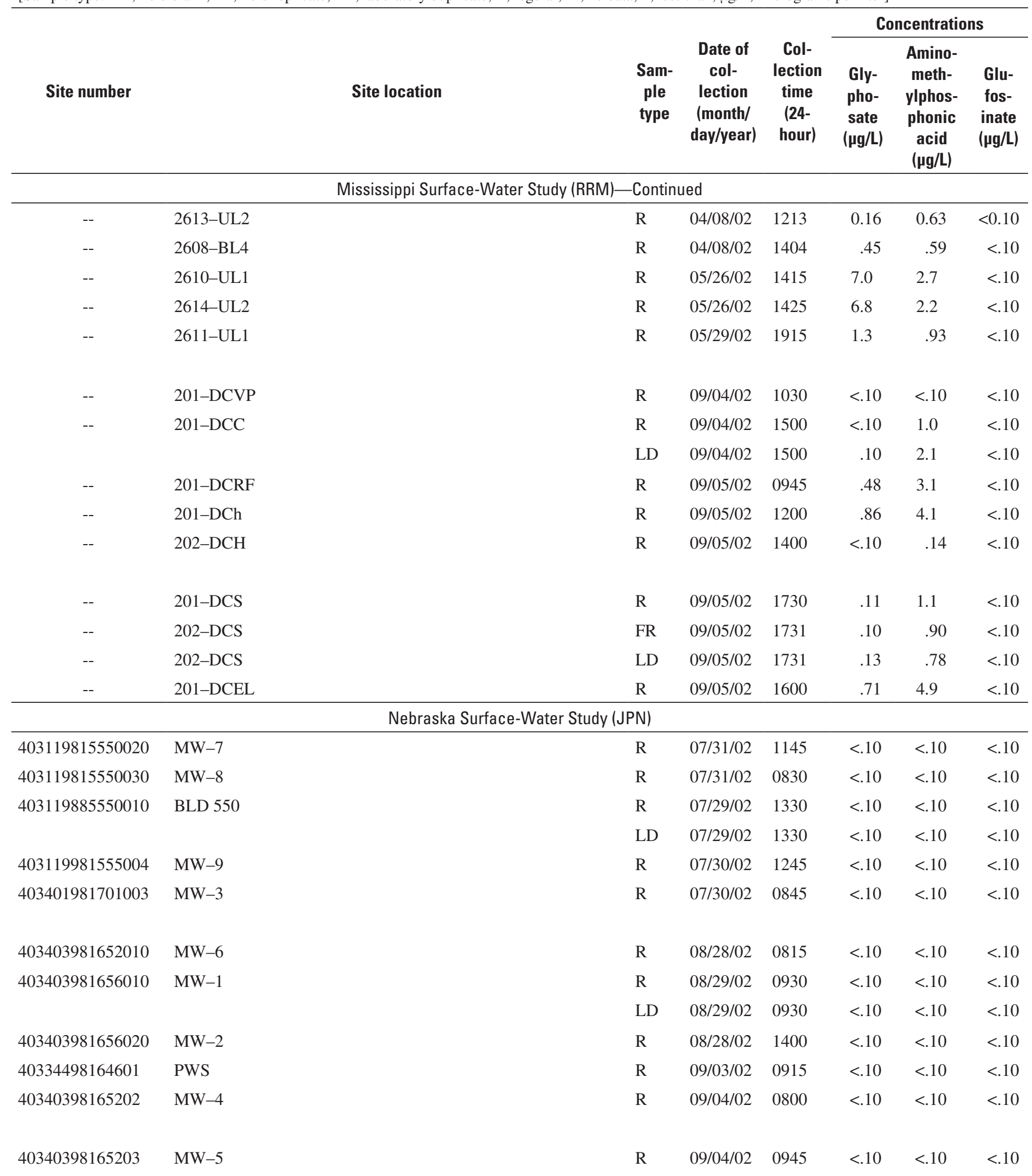


Table 3. Analytical results for glyphosate, aminomethylphosphonic acid, and glufosinate in State cooperative surface- and ground-water studies, 2001-06.-Continued

[Sample type: FB, field blank; FR, field replicate; LD, laboratory duplicate; R, regular; --, no data; <, less than; $\mu \mathrm{g} / \mathrm{L}$, micrograms per liter]

\begin{tabular}{|c|c|c|c|c|c|c|c|}
\hline \multirow[b]{2}{*}{ Site number } & \multirow[b]{2}{*}{ Site location } & \multirow[b]{2}{*}{$\begin{array}{c}\text { Sam- } \\
\text { ple } \\
\text { type }\end{array}$} & \multirow[b]{2}{*}{$\begin{array}{c}\text { Date of } \\
\text { col- } \\
\text { lection } \\
\text { (month/ } \\
\text { day/year) }\end{array}$} & \multirow[b]{2}{*}{$\begin{array}{l}\text { Col- } \\
\text { lection } \\
\text { time } \\
(24- \\
\text { hour })\end{array}$} & \multicolumn{3}{|c|}{ Concentrations } \\
\hline & & & & & $\begin{array}{c}\text { Gly- } \\
\text { pho- } \\
\text { sate } \\
(\mu \mathrm{g} / \mathrm{L})\end{array}$ & $\begin{array}{c}\text { Amino- } \\
\text { meth- } \\
\text { ylphos- } \\
\text { phonic } \\
\text { acid } \\
\text { ( } \mu \mathrm{g} / \mathrm{L})\end{array}$ & $\begin{array}{c}\text { Glu- } \\
\text { fos- } \\
\text { inate } \\
(\mu \mathrm{g} / \mathrm{L})\end{array}$ \\
\hline \multicolumn{8}{|c|}{ South Dakota Surface-Water Study (BSD) } \\
\hline \multirow[t]{2}{*}{425740098161100} & YST Wetlands Number 19 near Greenwood & $\mathrm{R}$ & $06 / 15 / 05$ & 0915 & 0.39 & 1.5 & $<0.02$ \\
\hline & & $\mathrm{R}$ & $06 / 15 / 05$ & 0945 & .08 & .14 & $<.02$ \\
\hline \multirow[t]{3}{*}{425941098173400} & YST Wetlands Number 18 near Wagner & $\mathrm{R}$ & $06 / 15 / 05$ & 0835 & .16 & .08 & $<.02$ \\
\hline & & $\mathrm{R}$ & $06 / 15 / 05$ & 1045 & .07 & .04 & $<.02$ \\
\hline & & $\mathrm{R}$ & $06 / 15 / 05$ & 1635 & .66 & .34 & $<.02$ \\
\hline 430125098120600 & YST Wetlands Number 17 near Dante & $\mathrm{R}$ & $06 / 13 / 05$ & 1540 & $<.02$ & .26 & $<.02$ \\
\hline \multirow[t]{2}{*}{430226098281400} & YST Wetlands Number 12 near Pickstown & $\mathrm{R}$ & $06 / 16 / 05$ & 0840 & $<.02$ & $<.02$ & $<.02$ \\
\hline & & FR & $06 / 16 / 05$ & 0845 & $<.02$ & $<.02$ & $<.02$ \\
\hline \multirow[t]{3}{*}{430300098253300} & YST Wetlands Number 8 near Pickstown & $\mathrm{R}$ & 06/15/05 & 1655 & .08 & .38 & $<.02$ \\
\hline & & $\mathrm{R}$ & $06 / 16 / 05$ & 0755 & $<.02$ & .29 & $<.02$ \\
\hline & & FR & $06 / 16 / 05$ & 0800 & .40 & .32 & $<.02$ \\
\hline 430311098253000 & YST Wetlands Number 23 near Ravinia & $\mathrm{R}$ & $06 / 16 / 05$ & 1650 & .18 & .36 & $<.02$ \\
\hline 430443098102200 & YST Wetlands Number 16 near Dry Choteau Creek near Dante & $\mathrm{R}$ & $06 / 13 / 05$ & 1215 & .07 & $<.02$ & $<.02$ \\
\hline 430500098064000 & YST Wetlands Number 15 near Dry Choteau Creek near Dante & FR & $06 / 13 / 05$ & 1445 & .10 & $<.02$ & $<.02$ \\
\hline \multirow[t]{2}{*}{430618098275800} & YST Wetlands Number 22 near Old Landfill near Ravinia & $\mathrm{R}$ & $06 / 16 / 05$ & 1255 & $<.02$ & $<.02$ & $<.02$ \\
\hline & & FR & 06/16/05 & 1300 & .07 & $<.02$ & $<.02$ \\
\hline \multirow[t]{2}{*}{430735098193000} & YST Wetlands Number 14 near Wagner & $\mathrm{R}$ & $06 / 13 / 05$ & 1115 & $<.02$ & .32 & $<.02$ \\
\hline & & $\mathrm{LD}$ & $06 / 13 / 05$ & 1115 & $<.02$ & .21 & $<.02$ \\
\hline 431116098355600 & YST Wetlands Number 6 near Lake Andes & $\mathrm{R}$ & $06 / 16 / 05$ & 1405 & .13 & $<.02$ & $<.02$ \\
\hline 431426098345800 & YST Wetlands Number 5 (Goose Lake) near Geddes & $\mathrm{R}$ & $06 / 16 / 05$ & 1455 & .19 & .20 & $<.02$ \\
\hline 431759098334000 & YST Wetlands Number 4 (WPA) near Geddes & $\mathrm{R}$ & $06 / 16 / 05$ & 1545 & $<.02$ & $<.02$ & $<.02$ \\
\hline \multirow[t]{2}{*}{431853098322600} & YST Wetlands Number 2 (Pheasant Acres) near Geddes & $\mathrm{R}$ & $06 / 14 / 05$ & 0830 & .10 & $<.02$ & $<.02$ \\
\hline & & $\mathrm{LD}$ & $06 / 14 / 05$ & 0830 & .09 & $<.02$ & $<.02$ \\
\hline 431907098321000 & YST Wetlands Number 3 (Pheasant Acres East) near Geddes & $\mathrm{R}$ & 06/14/05 & 0955 & .04 & $<.02$ & $<.02$ \\
\hline \multicolumn{8}{|c|}{ Vermont Surface-Water Study (ACV) } \\
\hline \multirow[t]{2}{*}{04282815} & Englesby Brook at Burlington & $\mathrm{R}$ & $06 / 26 / 06$ & 1200 & .08 & .05 & $<.02$ \\
\hline & & LD & $06 / 26 / 06$ & 1200 & .06 & .03 & $<.02$ \\
\hline 04282813 & Potash Brook at Queen City Park Road near Burlington & $\mathrm{R}$ & $06 / 26 / 06$ & 1245 & .03 & $<.02$ & $<.02$ \\
\hline 04292775 & Stevens Brook at Lower Newton Street at St. Albans & $\mathrm{R}$ & $06 / 26 / 06$ & 1445 & .62 & .09 & $<.02$ \\
\hline 04294000 & Missiquoi River at Swanton & $\mathrm{R}$ & $06 / 27 / 06$ & 1100 & $<.02$ & $<.02$ & $<.02$ \\
\hline
\end{tabular}


Table 3. Analytical results for glyphosate, aminomethylphosphonic acid, and glufosinate in State cooperative surface- and ground-water studies, 2001-06.-Continued

[Sample type: FB, field blank; FR, field replicate; LD, laboratory duplicate; R, regular; --, no data; <, less than; $\mu \mathrm{g} / \mathrm{L}$, micrograms per liter]

\begin{tabular}{|c|c|c|c|c|c|c|c|}
\hline \multirow[b]{2}{*}{ Site number } & \multirow[b]{2}{*}{ Site location } & \multirow[b]{2}{*}{$\begin{array}{c}\text { Sam- } \\
\text { ple } \\
\text { type }\end{array}$} & \multirow[b]{2}{*}{$\begin{array}{c}\text { Date of } \\
\text { col- } \\
\text { lection } \\
\text { (month/ } \\
\text { day/year) }\end{array}$} & \multirow[b]{2}{*}{$\begin{array}{l}\text { Col- } \\
\text { lection } \\
\text { time } \\
\text { (24- } \\
\text { hour) }\end{array}$} & \multicolumn{3}{|c|}{ Concentrations } \\
\hline & & & & & $\begin{array}{l}\text { Gly- } \\
\text { pho- } \\
\text { sate } \\
\text { ( } \mu \mathrm{g} / \mathrm{L})\end{array}$ & $\begin{array}{l}\text { Amino- } \\
\text { meth- } \\
\text { ylphos- } \\
\text { phonic } \\
\text { acid } \\
\text { ( } \mathrm{gg} / \mathrm{L} \text { ) }\end{array}$ & $\begin{array}{c}\text { Glu- } \\
\text { fos- } \\
\text { inate } \\
(\mu \mathrm{g} / \mathrm{L})\end{array}$ \\
\hline \multicolumn{8}{|c|}{ Washington Surface-Water Study (LMW) } \\
\hline \multirow[t]{4}{*}{12113499} & Taylor Creek at Lakeridge Park near Renton & $\mathrm{R}$ & $07 / 08 / 03$ & 1305 & $<0.10$ & $<0.10$ & $<0.10$ \\
\hline & & $\mathrm{R}$ & $08 / 05 / 03$ & 1300 & $<.10$ & $<.10$ & $<.10$ \\
\hline & & $\mathrm{R}$ & $10 / 18 / 03$ & 0730 & .16 & $<.10$ & $<.10$ \\
\hline & & $\mathrm{R}$ & $11 / 18 / 03$ & 1145 & .10 & $<.10$ & $<.10$ \\
\hline \multirow[t]{4}{*}{12119600} & May Creek at mouth near Renton & $\mathrm{R}$ & 07/08/03 & 1110 & $<.10$ & $<.10$ & $<.10$ \\
\hline & & $\mathrm{R}$ & 08/05/03 & 1105 & $<.10$ & $<.10$ & $<.10$ \\
\hline & & $\mathrm{R}$ & $10 / 16 / 03$ & 1130 & .23 & $<.10$ & $<.10$ \\
\hline & & $\mathrm{R}$ & $11 / 18 / 03$ & 1100 & .21 & $<.10$ & $<.10$ \\
\hline \multirow[t]{5}{*}{12120000} & Mercer Creek near Bellevue & $\mathrm{R}$ & 07/08/03 & 1505 & .43 & .14 & $<.10$ \\
\hline & & $\mathrm{R}$ & $08 / 05 / 03$ & 1145 & $<.10$ & .10 & $<.10$ \\
\hline & & $\mathrm{R}$ & $10 / 16 / 03$ & 0930 & .48 & $<.10$ & $<.10$ \\
\hline & & $\mathrm{R}$ & $11 / 18 / 03$ & 1000 & .68 & $<.10$ & $<.10$ \\
\hline & & LD & $11 / 18 / 03$ & 1000 & .59 & $<.10$ & $<.10$ \\
\hline \multirow[t]{4}{*}{12125500} & Bear Creek at Woodinville & $\mathrm{R}$ & $06 / 28 / 02$ & 2145 & 2.0 & .38 & $<.10$ \\
\hline & & $\mathrm{R}$ & 07/10/02 & 1115 & $<.10$ & $<.10$ & $<.10$ \\
\hline & & $\mathrm{R}$ & $08 / 21 / 02$ & 1130 & .12 & .10 & $<.10$ \\
\hline & & $\mathrm{R}$ & $11 / 12 / 02$ & 1230 & .31 & .15 & $<.10$ \\
\hline \multirow[t]{6}{*}{12126200} & North Creek at North Creek Parkway near Bothell & $\mathrm{R}$ & $06 / 28 / 02$ & 2245 & .10 & $<.10$ & $<.10$ \\
\hline & & $\mathrm{R}$ & $07 / 10 / 02$ & 1300 & $<.10$ & $<.10$ & $<.10$ \\
\hline & & $\mathrm{R}$ & $08 / 21 / 02$ & 1215 & $<.10$ & $<.10$ & $<.10$ \\
\hline & & $\mathrm{R}$ & $11 / 12 / 02$ & 1300 & $<.10$ & $<.10$ & $<.10$ \\
\hline & & FB & $11 / 12 / 02$ & 1305 & $<.10$ & $<.10$ & $<.10$ \\
\hline & & LD & $11 / 12 / 02$ & 1305 & $<.10$ & $<.10$ & $<.10$ \\
\hline \multirow[t]{7}{*}{474243122083001} & Sammamish River Irrigation Return at 124th Street & $\mathrm{R}$ & $06 / 28 / 02$ & 2045 & 1.2 & .52 & $<.10$ \\
\hline & & LD & $06 / 28 / 02$ & 2045 & .97 & .53 & $<.10$ \\
\hline & & $\mathrm{R}$ & $07 / 10 / 02$ & 1000 & .79 & .82 & $<.10$ \\
\hline & & $\mathrm{R}$ & $08 / 21 / 02$ & 0945 & 1.3 & 1.3 & $<.10$ \\
\hline & & $\mathrm{LD}$ & $08 / 21 / 02$ & 0945 & .86 & 1.7 & $<.10$ \\
\hline & & $\mathrm{R}$ & $11 / 12 / 02$ & 1200 & .84 & .48 & $<.10$ \\
\hline & & LD & $11 / 12 / 02$ & 1200 & .80 & .45 & $<.10$ \\
\hline
\end{tabular}


Table 4. Analytical results for glyphosate, aminomethylphosphonic acid, and glufosinate in National Stream Quality Accounting Network Program (NASQAN) surface- and ground-water studies, 2002.

[ Sample type: FR, field replicate; R, regular; LD, laboratory duplicate; --, no data; <, less than; $\mu \mathrm{g} / \mathrm{L}$, micrograms per liter]

\begin{tabular}{|c|c|c|c|c|c|c|c|}
\hline \multirow[b]{2}{*}{$\begin{array}{c}\text { Site } \\
\text { number } \\
\text { (fig. 3) }\end{array}$} & \multirow[b]{2}{*}{$\begin{array}{c}\text { Site } \\
\text { Iocation }\end{array}$} & \multirow[b]{2}{*}{$\begin{array}{l}\text { Sam- } \\
\text { ple } \\
\text { type }\end{array}$} & \multirow[b]{2}{*}{$\begin{array}{c}\text { Date of } \\
\text { collec- } \\
\text { tion (day/ } \\
\text { month/ } \\
\text { year) }\end{array}$} & \multirow[b]{2}{*}{$\begin{array}{l}\text { Col- } \\
\text { lection } \\
\text { time } \\
\text { (24- } \\
\text { hour) }\end{array}$} & \multicolumn{3}{|c|}{ Concentrations } \\
\hline & & & & & $\begin{array}{c}\text { Glypho- } \\
\text { sate } \\
\text { ( } \mu \mathrm{g} / \mathrm{L})\end{array}$ & $\begin{array}{c}\text { Amino- } \\
\text { meth- } \\
\text { ylphos- } \\
\text { phonic } \\
\text { acid } \\
\text { ( } \mu \mathrm{g} / \mathrm{L})\end{array}$ & $\begin{array}{c}\text { Glufos- } \\
\text { inate } \\
\text { ( } \mu \mathrm{g} / \mathrm{L})\end{array}$ \\
\hline \multirow{6}{*}{03612500} & & $\mathrm{R}$ & $05 / 29 / 02$ & 1400 & $<.10$ & $<.10$ & $<.10$ \\
\hline & & $\mathrm{R}$ & $06 / 12 / 02$ & 1510 & $<.10$ & .15 & $<.10$ \\
\hline & & $\mathrm{R}$ & $06 / 26 / 02$ & 1330 & $<.10$ & $<.10$ & $<.10$ \\
\hline & & $\mathrm{R}$ & 09/10/02 & 1410 & $<.10$ & .16 & $<.10$ \\
\hline & & LD & $09 / 10 / 02$ & 1410 & $<.10$ & .16 & $<.10$ \\
\hline & & FR & 09/10/02 & 1420 & $<.10$ & .17 & $<.10$ \\
\hline \multirow[t]{6}{*}{07022000} & Mississippi River at Thebes, Illinois & $\mathrm{R}$ & $04 / 29 / 02$ & 1410 & $<.10$ & .19 & $<.10$ \\
\hline & & $\mathrm{R}$ & $05 / 09 / 02$ & 1010 & $<.10$ & $<.10$ & $<.10$ \\
\hline & & $\mathrm{R}$ & $05 / 14 / 02$ & 1050 & $<.10$ & .13 & $<.10$ \\
\hline & & $\mathrm{R}$ & $06 / 12 / 02$ & 1300 & $<.10$ & .32 & $<.10$ \\
\hline & & $\mathrm{R}$ & 07/10/02 & 1510 & $<.10$ & .27 & $<.10$ \\
\hline & & $\mathrm{LD}$ & $10 / 23 / 02$ & 1355 & $<.10$ & .12 & $<.10$ \\
\hline \multirow[t]{4}{*}{07288955} & Yazoo River below Steele Bayou near Long Lake, Mississippi & $\mathrm{R}$ & $04 / 12 / 02$ & 1200 & .33 & $<.10$ & $<.10$ \\
\hline & & $\mathrm{R}$ & 05/10/02 & 1200 & $<.10$ & .17 & $<.10$ \\
\hline & & $\mathrm{R}$ & $06 / 27 / 02$ & 1200 & .14 & .38 & $<.10$ \\
\hline & & $\mathrm{R}$ & 07/09/02 & 1200 & $<.10$ & $<.10$ & $<.10$ \\
\hline \multirow[t]{6}{*}{07373420} & Mississippi River near St. Francisville, Mississippi & $\mathrm{R}$ & $04 / 22 / 02$ & 1130 & $<.10$ & $<.10$ & $<.10$ \\
\hline & & $\mathrm{R}$ & $05 / 07 / 02$ & 1030 & $<.10$ & .12 & $<.10$ \\
\hline & & $\mathrm{R}$ & $05 / 28 / 02$ & 1000 & $<.10$ & $<.10$ & $<.10$ \\
\hline & & $\mathrm{R}$ & $08 / 05 / 02$ & -- & $<.10$ & .25 & $<.10$ \\
\hline & & $\mathrm{R}$ & $08 / 19 / 02$ & 1000 & $<.10$ & .20 & $<.10$ \\
\hline & & $\mathrm{R}$ & $09 / 09 / 02$ & 1000 & $<.10$ & .23 & $<.10$ \\
\hline
\end{tabular}


Table 5. Analytical results for glyphosate, aminomethylphosphonic acid, and glufosinate in National Water-Quality Assessment (NAWQA) Program surface- and ground-water studies, 2001-06.

[Location of NAWQA study units shown in figure 4. Sample type: FB, field blank; FR, field replicate; R, regular; LD, laboratory duplicate; --, no data; <, less than; $\mu \mathrm{g} / \mathrm{L}$, micrograms per liter]

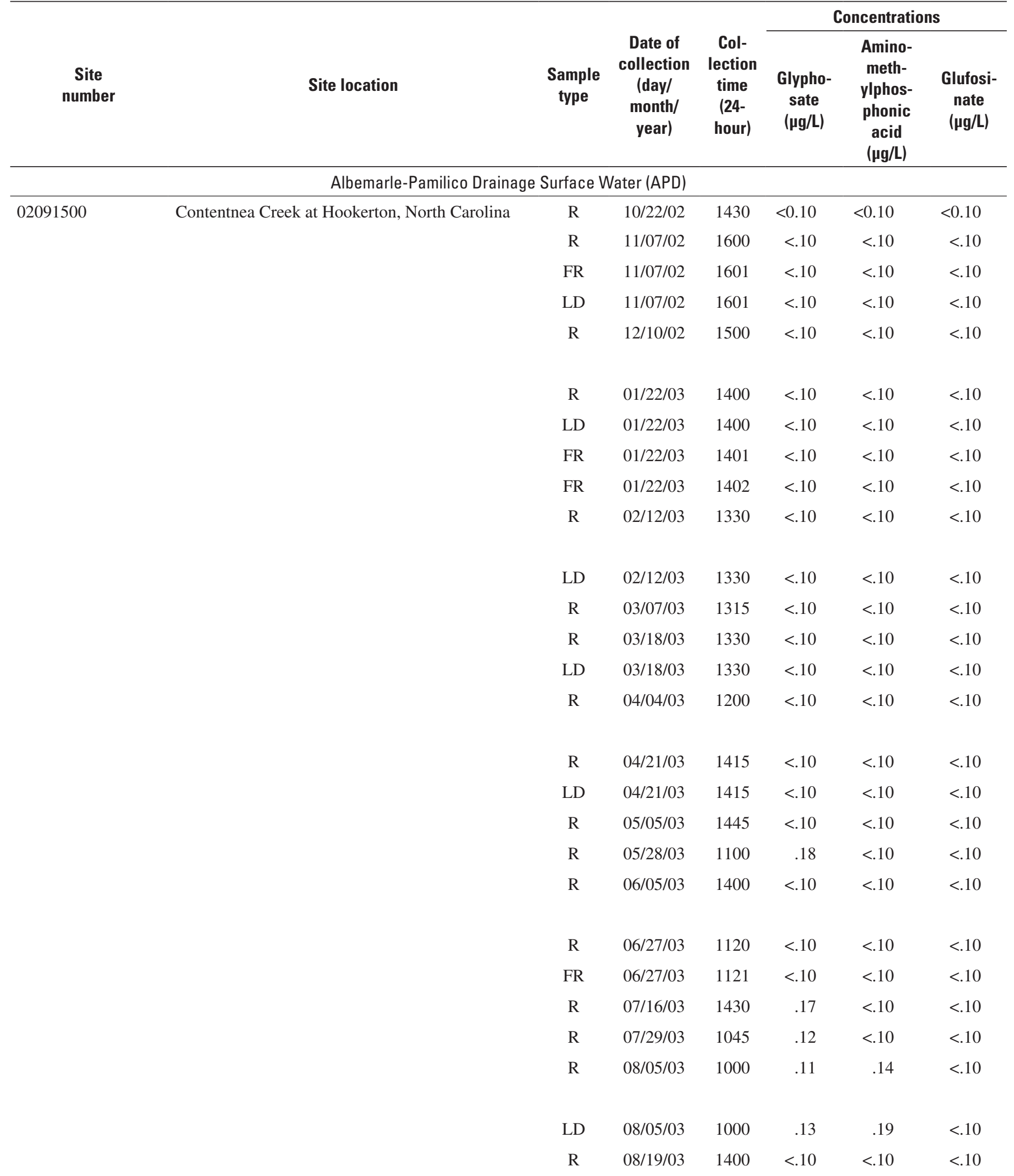


Table 5. Analytical results for glyphosate, aminomethylphosphonic acid, and glufosinate in National Water-Quality Assessment (NAWQA) Program surface- and ground-water studies, 2001-06.-Continued

[Location of NAWQA study units shown in figure 4. Sample type: FB, field blank; FR, field replicate; R, regular; LD, laboratory duplicate; --, no data; <, less than; $\mu \mathrm{g} / \mathrm{L}$, micrograms per liter]

\begin{tabular}{|c|c|c|c|c|c|c|c|}
\hline \multirow[b]{2}{*}{$\begin{array}{c}\text { Site } \\
\text { number }\end{array}$} & \multirow[b]{2}{*}{ Site location } & \multirow[b]{2}{*}{$\begin{array}{c}\text { Sample } \\
\text { type }\end{array}$} & \multirow[b]{2}{*}{$\begin{array}{c}\text { Date of } \\
\text { collection } \\
\text { (day/ } \\
\text { month/ } \\
\text { year) }\end{array}$} & \multirow[b]{2}{*}{$\begin{array}{l}\text { Col- } \\
\text { lection } \\
\text { time } \\
\text { (24- } \\
\text { hour) }\end{array}$} & \multicolumn{3}{|c|}{ Concentrations } \\
\hline & & & & & $\begin{array}{c}\text { Glypho- } \\
\text { sate } \\
\text { ( } \mu \mathrm{g} / \mathrm{L})\end{array}$ & $\begin{array}{c}\text { Amino- } \\
\text { meth- } \\
\text { ylphos- } \\
\text { phonic } \\
\text { acid } \\
\text { ( } \mu \mathrm{g} / \mathrm{L})\end{array}$ & $\begin{array}{c}\text { Glufosi- } \\
\text { nate } \\
\text { ( } \mu \mathrm{g} / \mathrm{L})\end{array}$ \\
\hline \multicolumn{8}{|c|}{ Albemarle-Pamilico Drainage Surface Water (APD)_Continued } \\
\hline \multirow[t]{3}{*}{02091500} & Contentnea Creek at Hookerton, North Carolina & FR & $08 / 19 / 03$ & 1401 & $<0.10$ & $<0.10$ & $<0.10$ \\
\hline & & $\mathrm{R}$ & 09/02/03 & 1415 & $<.10$ & $<.10$ & $<.10$ \\
\hline & & LD & $09 / 02 / 03$ & 1415 & $<.10$ & $<.10$ & $<.10$ \\
\hline \multicolumn{8}{|c|}{ Albemarle-Pamilico Drainage Ground Water (APG) } \\
\hline 345516077190001 & LU-20 & $\mathrm{R}$ & $04 / 02 / 02$ & 1000 & $<.10$ & $<.10$ & $<.10$ \\
\hline 345630077163501 & LU-S20B & $\mathrm{R}$ & $04 / 02 / 02$ & 1130 & $<.10$ & $<.10$ & $<.10$ \\
\hline 352305077321701 & LU-15 & $\mathrm{R}$ & $04 / 18 / 02$ & 1000 & $<.10$ & $<.10$ & $<.10$ \\
\hline 352548077012701 & LU-14 & $\mathrm{R}$ & 04/17/02 & 1000 & $<.10$ & $<.10$ & $<.10$ \\
\hline 352702077302501 & LU-S15A & $\mathrm{R}$ & $04 / 04 / 02$ & 1200 & $<.10$ & $<.10$ & $<.10$ \\
\hline \multirow[t]{2}{*}{352905077594501} & LU-013 & $\mathrm{R}$ & $03 / 28 / 02$ & 1015 & $<.10$ & $<.10$ & $<.10$ \\
\hline & & LD & 03/28/02 & 1015 & $<.10$ & $<.10$ & $<.10$ \\
\hline 353111077334402 & & LD & $04 / 29 / 02$ & 1130 & $<.10$ & $<.10$ & $<.10$ \\
\hline 353148077332103 & L11S & $\mathrm{R}$ & $04 / 29 / 02$ & 1300 & $<.10$ & $<.10$ & $<.10$ \\
\hline 353219077153801 & PI-532 & $\mathrm{R}$ & 05/09/02 & 1115 & $<.10$ & $<.10$ & $<.10$ \\
\hline 353221076105501 & LU-S9C & $\mathrm{R}$ & $04 / 15 / 02$ & 1245 & $<.10$ & $<.10$ & $<.10$ \\
\hline 353547076473301 & LU-10A & $\mathrm{R}$ & $04 / 16 / 02$ & 1430 & $<.10$ & $<.10$ & $<.10$ \\
\hline \multirow[t]{2}{*}{035473307729802} & ED-174 & $\mathrm{R}$ & 06/07/02 & 1130 & $<.10$ & $<.10$ & $<.10$ \\
\hline & & LD & 06/07/02 & 1130 & $<.10$ & $<.10$ & $<.10$ \\
\hline 354750076344501 & LU-08 & $\mathrm{R}$ & $04 / 16 / 02$ & 1200 & $<.10$ & $<.10$ & $<.10$ \\
\hline 355601076352401 & LU-07A & $\mathrm{R}$ & $04 / 16 / 02$ & 1015 & $<.10$ & $<.10$ & $<.10$ \\
\hline 361420077111401 & BE-080 & $\mathrm{R}$ & 05/14/02 & 1215 & $<.10$ & $<.10$ & $<.10$ \\
\hline
\end{tabular}


Table 5. Analytical results for glyphosate, aminomethylphosphonic acid, and glufosinate in National Water-Quality Assessment (NAWQA) Program surface- and ground-water studies, 2001-06.-Continued

[Location of NAWQA study units shown in figure 4. Sample type: FB, field blank; FR, field replicate; R, regular; LD, laboratory duplicate; --, no data; <, less than; $\mu \mathrm{g} / \mathrm{L}$, micrograms per liter]

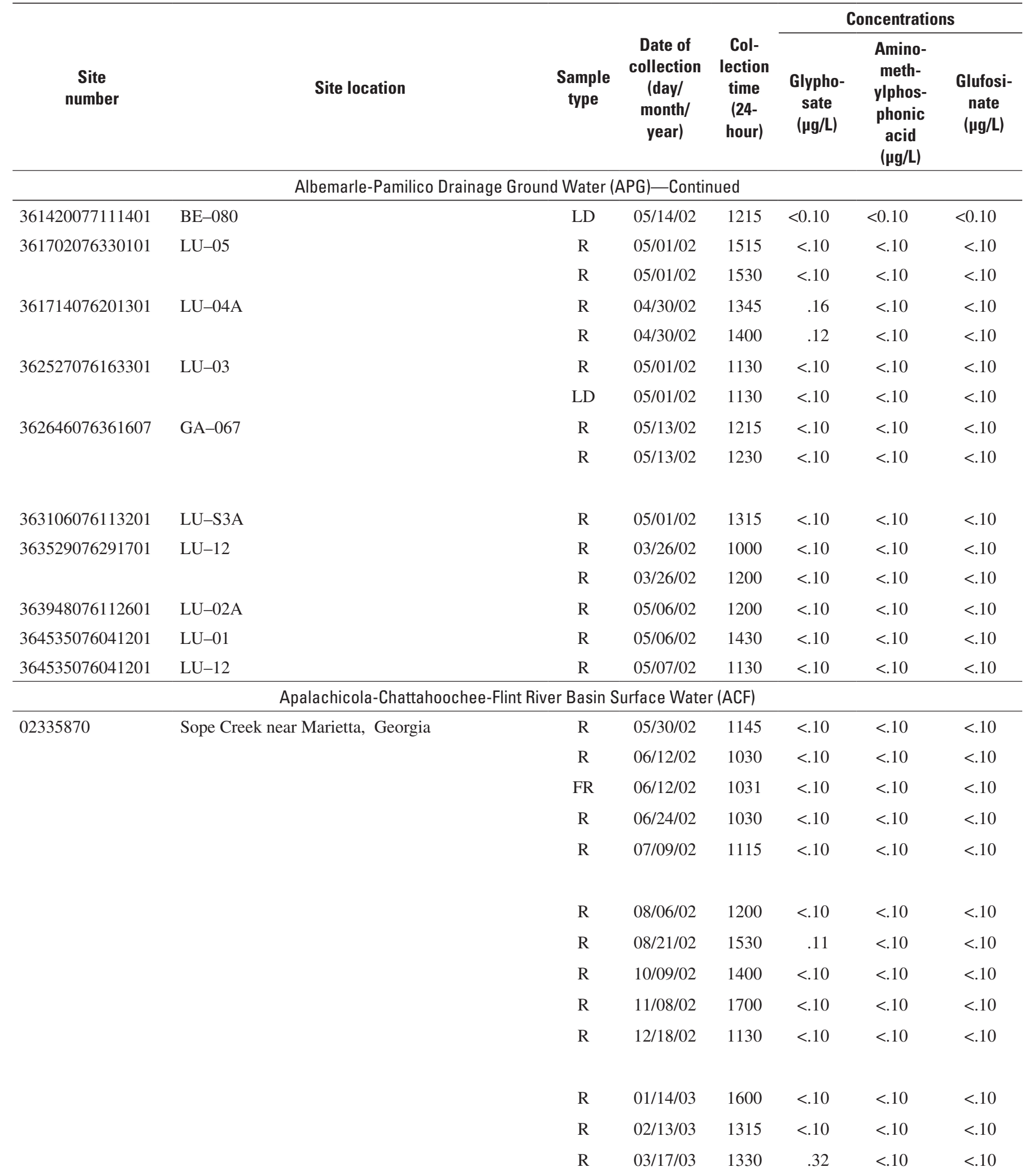


Table 5. Analytical results for glyphosate, aminomethylphosphonic acid, and glufosinate in National Water-Quality Assessment (NAWQA) Program surface- and ground-water studies, 2001-06.-Continued

[Location of NAWQA study units shown in figure 4. Sample type: FB, field blank; FR, field replicate; R, regular; LD, laboratory duplicate; --, no data; <, less than; $\mu \mathrm{g} / \mathrm{L}$, micrograms per liter]

\begin{tabular}{|c|c|c|c|c|c|c|c|}
\hline \multirow[b]{2}{*}{$\begin{array}{c}\text { Site } \\
\text { number }\end{array}$} & \multirow[b]{2}{*}{ Site location } & \multirow[b]{2}{*}{$\begin{array}{c}\text { Sample } \\
\text { type }\end{array}$} & \multirow[b]{2}{*}{$\begin{array}{c}\text { Date of } \\
\text { collection } \\
\text { (day/ } \\
\text { month/ } \\
\text { year) }\end{array}$} & \multirow[b]{2}{*}{$\begin{array}{l}\text { Col- } \\
\text { lection } \\
\text { time } \\
\text { (24- } \\
\text { hour) }\end{array}$} & \multicolumn{3}{|c|}{ Concentrations } \\
\hline & & & & & $\begin{array}{c}\text { Glypho- } \\
\text { sate } \\
\text { ( } \mu \mathrm{g} / \mathrm{L})\end{array}$ & $\begin{array}{c}\text { Amino- } \\
\text { meth- } \\
\text { ylphos- } \\
\text { phonic } \\
\text { acid } \\
(\mu \mathrm{g} / \mathrm{L})\end{array}$ & $\begin{array}{c}\text { Glufosi- } \\
\text { nate } \\
\text { ( } \mu \mathrm{g} / \mathrm{L})\end{array}$ \\
\hline \multirow[t]{11}{*}{02335870} & Sope Creek near Marietta, Georgia-Continued & FR & $03 / 17 / 03$ & 1337 & 0.38 & $<0.10$ & $<0.10$ \\
\hline & & $\mathrm{R}$ & $04 / 04 / 03$ & 1315 & $<.10$ & $<.10$ & $<.10$ \\
\hline & & $\mathrm{R}$ & $04 / 15 / 03$ & 1400 & $<.10$ & $<.10$ & $<.10$ \\
\hline & & $\mathrm{R}$ & $05 / 01 / 03$ & 1030 & $<.10$ & $<.10$ & $<.10$ \\
\hline & & $\mathrm{R}$ & $05 / 28 / 03$ & 1345 & $<.10$ & $<.10$ & $<.10$ \\
\hline & & $\mathrm{R}$ & $06 / 16 / 03$ & 1130 & $<.10$ & $<.10$ & $<.10$ \\
\hline & & $\mathrm{R}$ & $07 / 01 / 03$ & 1415 & .11 & $<.10$ & $<.10$ \\
\hline & & FR & 07/01/03 & 1417 & $<.10$ & $<.10$ & $<.10$ \\
\hline & & $\mathrm{R}$ & $07 / 14 / 03$ & 1545 & $<.10$ & .11 & $<.10$ \\
\hline & & $\mathrm{R}$ & $07 / 30 / 03$ & 0830 & $<.10$ & .15 & $<.10$ \\
\hline & & $\mathrm{R}$ & $08 / 11 / 03$ & 1130 & .13 & .11 & $<.10$ \\
\hline \multirow[t]{2}{*}{02338523} & Hillabahatchee Creek at Franklin Road near & $\mathrm{R}$ & $06 / 12 / 02$ & 1500 & $<.10$ & $<.10$ & $<.10$ \\
\hline & Franklin, Georgia & $\mathrm{R}$ & 07/10/02 & 1500 & $<.10$ & $<.10$ & $<.10$ \\
\hline \multirow[t]{8}{*}{02350080} & Lime Creek near Cobb, Georgia & $\mathrm{R}$ & $05 / 31 / 02$ & 0930 & $<.10$ & $<.10$ & $<.10$ \\
\hline & & $\mathrm{R}$ & $06 / 05 / 02$ & -- & $<.10$ & $<.10$ & $<.10$ \\
\hline & & $\mathrm{R}$ & $06 / 13 / 02$ & 0900 & $<.10$ & $<.10$ & $<.10$ \\
\hline & & $\mathrm{R}$ & $06 / 26 / 02$ & 0900 & $<.10$ & $<.10$ & $<.10$ \\
\hline & & $\mathrm{LD}$ & $06 / 26 / 02$ & 0900 & $<.10$ & $<.10$ & $<.10$ \\
\hline & & LD & $07 / 11 / 02$ & 0915 & $<.10$ & .10 & $<.10$ \\
\hline & & $\mathrm{R}$ & $07 / 11 / 02$ & 0915 & $<.10$ & .10 & $<.10$ \\
\hline & & $\mathrm{R}$ & $08 / 22 / 02$ & 0900 & $<.10$ & $<.10$ & $<.10$ \\
\hline
\end{tabular}


Table 5. Analytical results for glyphosate, aminomethylphosphonic acid, and glufosinate in National Water-Quality Assessment (NAWQA) Program surface- and ground-water studies, 2001-06.-Continued

[Location of NAWQA study units shown in figure 4. Sample type: FB, field blank; FR, field replicate; R, regular; LD, laboratory duplicate; --, no data; <, less than; $\mu \mathrm{g} / \mathrm{L}$, micrograms per liter]

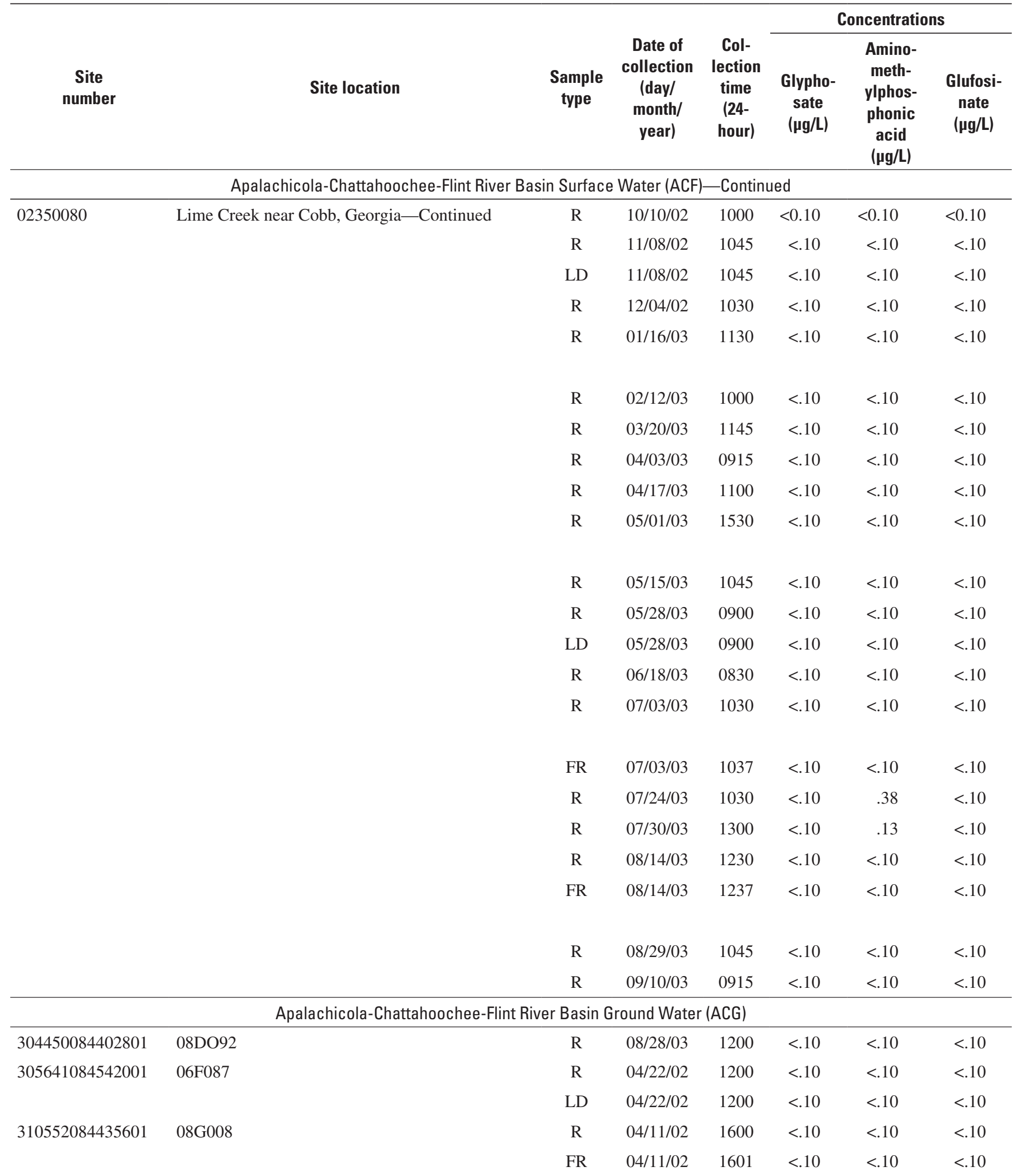


Table 5. Analytical results for glyphosate, aminomethylphosphonic acid, and glufosinate in National Water-Quality Assessment (NAWQA) Program surface- and ground-water studies, 2001-06.-Continued

[Location of NAWQA study units shown in figure 4. Sample type: FB, field blank; FR, field replicate; R, regular; LD, laboratory duplicate; --, no data; <, less than; $\mu \mathrm{g} / \mathrm{L}$, micrograms per liter]

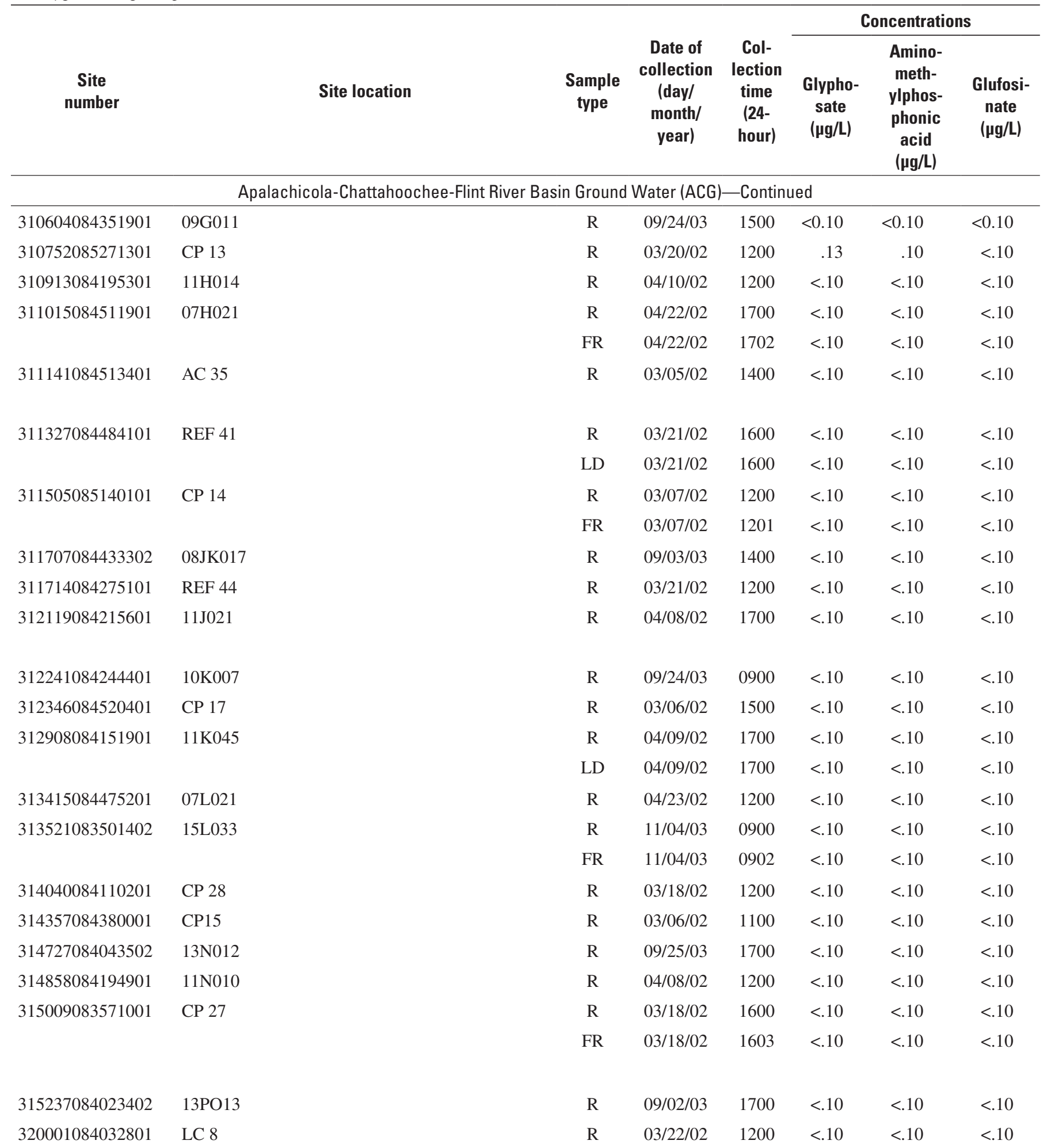


Table 5. Analytical results for glyphosate, aminomethylphosphonic acid, and glufosinate in National Water-Quality Assessment (NAWQA) Program surface- and ground-water studies, 2001-06.-Continued

[Location of NAWQA study units shown in figure 4. Sample type: FB, field blank; FR, field replicate; R, regular; LD, laboratory duplicate; --, no data; <, less than; $\mu \mathrm{g} / \mathrm{L}$, micrograms per liter]

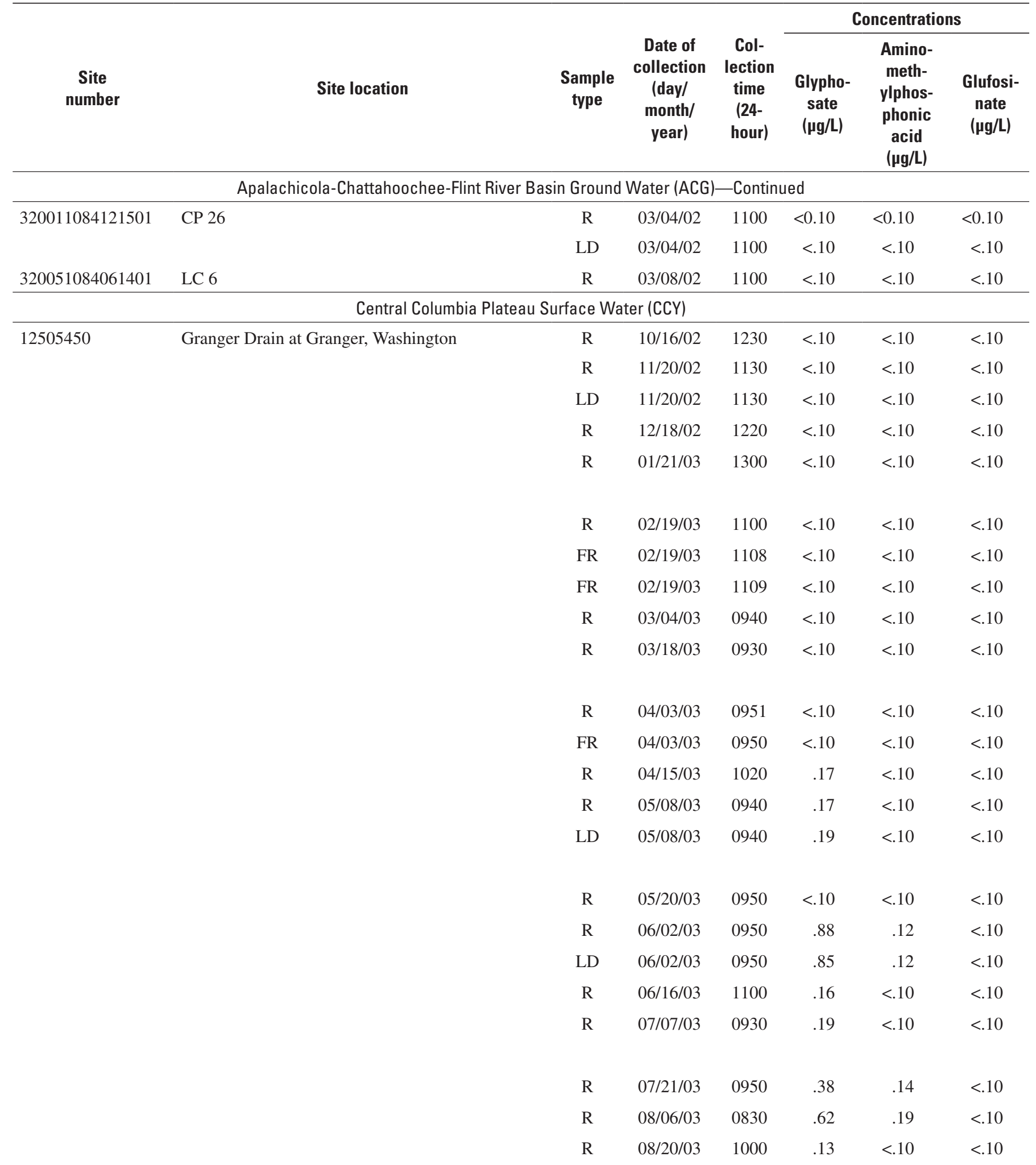


Table 5. Analytical results for glyphosate, aminomethylphosphonic acid, and glufosinate in National Water-Quality Assessment (NAWQA) Program surface- and ground-water studies, 2001-06.-Continued

[Location of NAWQA study units shown in figure 4. Sample type: FB, field blank; FR, field replicate; R, regular; LD, laboratory duplicate; --, no data; <, less than; $\mu \mathrm{g} / \mathrm{L}$, micrograms per liter]

\begin{tabular}{|c|c|c|c|c|c|c|c|}
\hline \multirow[b]{2}{*}{$\begin{array}{c}\text { Site } \\
\text { number }\end{array}$} & \multirow[b]{2}{*}{ Site location } & \multirow[b]{2}{*}{$\begin{array}{l}\text { Sample } \\
\text { type }\end{array}$} & \multirow[b]{2}{*}{$\begin{array}{c}\text { Date of } \\
\text { collection } \\
\text { (day/ } \\
\text { month/ } \\
\text { year) }\end{array}$} & \multirow[b]{2}{*}{$\begin{array}{c}\text { Col- } \\
\text { lection } \\
\text { time } \\
(24- \\
\text { hour })\end{array}$} & \multicolumn{3}{|c|}{ Concentrations } \\
\hline & & & & & $\begin{array}{c}\text { Glypho- } \\
\text { sate } \\
\text { ( } \mathrm{gg} / \mathrm{L})\end{array}$ & $\begin{array}{c}\text { Amino- } \\
\text { meth- } \\
\text { ylphos- } \\
\text { phonic } \\
\text { acid } \\
\text { ( } \mu \mathrm{g} / \mathrm{L})\end{array}$ & $\begin{array}{c}\text { Glufosi- } \\
\text { nate } \\
(\mu \mathrm{g} / \mathrm{L})\end{array}$ \\
\hline \multicolumn{8}{|c|}{ Central Columbia Plateau Surface Water (CCY)—Continued } \\
\hline 12505450 & Granger Drain at Granger, Washington & $\mathrm{R}$ & $09 / 16 / 03$ & 0850 & $<0.10$ & $<0.10$ & $<0.10$ \\
\hline 12510500 & Yakima River at Kiona, Washington & $\mathrm{R}$ & $10 / 15 / 02$ & 1140 & $<.10$ & $<.10$ & $<.10$ \\
\hline 462023120075200 & $\begin{array}{l}\text { DR2 at Yakima Valley Highway near Granger, } \\
\text { Washington }\end{array}$ & $\mathrm{R}$ & $09 / 16 / 03$ & 1320 & .11 & $<.10$ & $<.10$ \\
\hline \multicolumn{8}{|c|}{ Central Nebraska Basin Surface Water (CNB) } \\
\hline 06799750 & K7 & $\mathrm{R}$ & $03 / 25 / 04$ & 1115 & $<.02$ & $<.02$ & $<.02$ \\
\hline 06799750 & K7 (fall) & $\mathrm{R}$ & 05/08/04 & 2320 & .65 & 1.6 & .13 \\
\hline 06799750 & K7 (fall) & LD & 05/08/04 & 2320 & .65 & 1.6 & $<.02$ \\
\hline 06799750 & K7 (composite) & $\mathrm{R}$ & $05 / 10 / 04$ & 0100 & .24 & .76 & .13 \\
\hline 06799750 & K7 (rise) & $\mathrm{R}$ & 05/10/04 & 0148 & .17 & .85 & $<.02$ \\
\hline 06799750 & K7 (peak) & $\mathrm{R}$ & 05/10/04 & 0244 & .26 & .96 & $<.02$ \\
\hline 06799750 & K7 (fall) & $\mathrm{R}$ & 05/10/04 & 0446 & .27 & 1.1 & .15 \\
\hline 06799750 & K7 (composite) & $\mathrm{R}$ & 05/13/04 & 0450 & .15 & .80 & $<.02$ \\
\hline 06799750 & K7 (rise/peak) & $\mathrm{R}$ & 05/13/04 & 0554 & .14 & .66 & $<.02$ \\
\hline 06799750 & K7 (fall) & $\mathrm{R}$ & 05/13/04 & 0739 & .26 & .76 & $<.02$ \\
\hline 06799750 & K7 (fall) & LD & 05/13/04 & 0739 & .26 & .76 & $<.02$ \\
\hline \multirow[t]{11}{*}{06799750} & K7 & $\mathrm{R}$ & $05 / 22 / 04$ & 1020 & .22 & .66 & $<.02$ \\
\hline & & $\mathrm{R}$ & $05 / 22 / 04$ & 1831 & .10 & .50 & $<.02$ \\
\hline & & $\mathrm{R}$ & 05/22/04 & 1930 & .05 & .40 & $<.02$ \\
\hline & & $\mathrm{R}$ & $05 / 22 / 04$ & 2047 & .17 & .74 & $<.02$ \\
\hline & & $\mathrm{R}$ & 06/02/04 & 2030 & .06 & .34 & $<.02$ \\
\hline & & $\mathrm{R}$ & 06/16/04 & 1026 & .65 & .70 & $<.02$ \\
\hline & & LD & 06/16/04 & 1026 & .70 & .52 & $<.02$ \\
\hline & & $\mathrm{R}$ & 06/29/04 & 1010 & 1.0 & 1.2 & $<.02$ \\
\hline & & FR & 06/29/04 & 1020 & 1.4 & 1.0 & $<.02$ \\
\hline & & $\mathrm{R}$ & 07/07/04 & 1447 & 4.6 & 5.4 & $<.02$ \\
\hline & & FR & 07/07/04 & 1500 & 1.0 & 1.4 & $<.02$ \\
\hline
\end{tabular}


Table 5. Analytical results for glyphosate, aminomethylphosphonic acid, and glufosinate in National Water-Quality Assessment (NAWQA) Program surface- and ground-water studies, 2001-06.-Continued

[Location of NAWQA study units shown in figure 4. Sample type: FB, field blank; FR, field replicate; R, regular; LD, laboratory duplicate; --, no data; <, less than; $\mu \mathrm{g} / \mathrm{L}$, micrograms per liter]

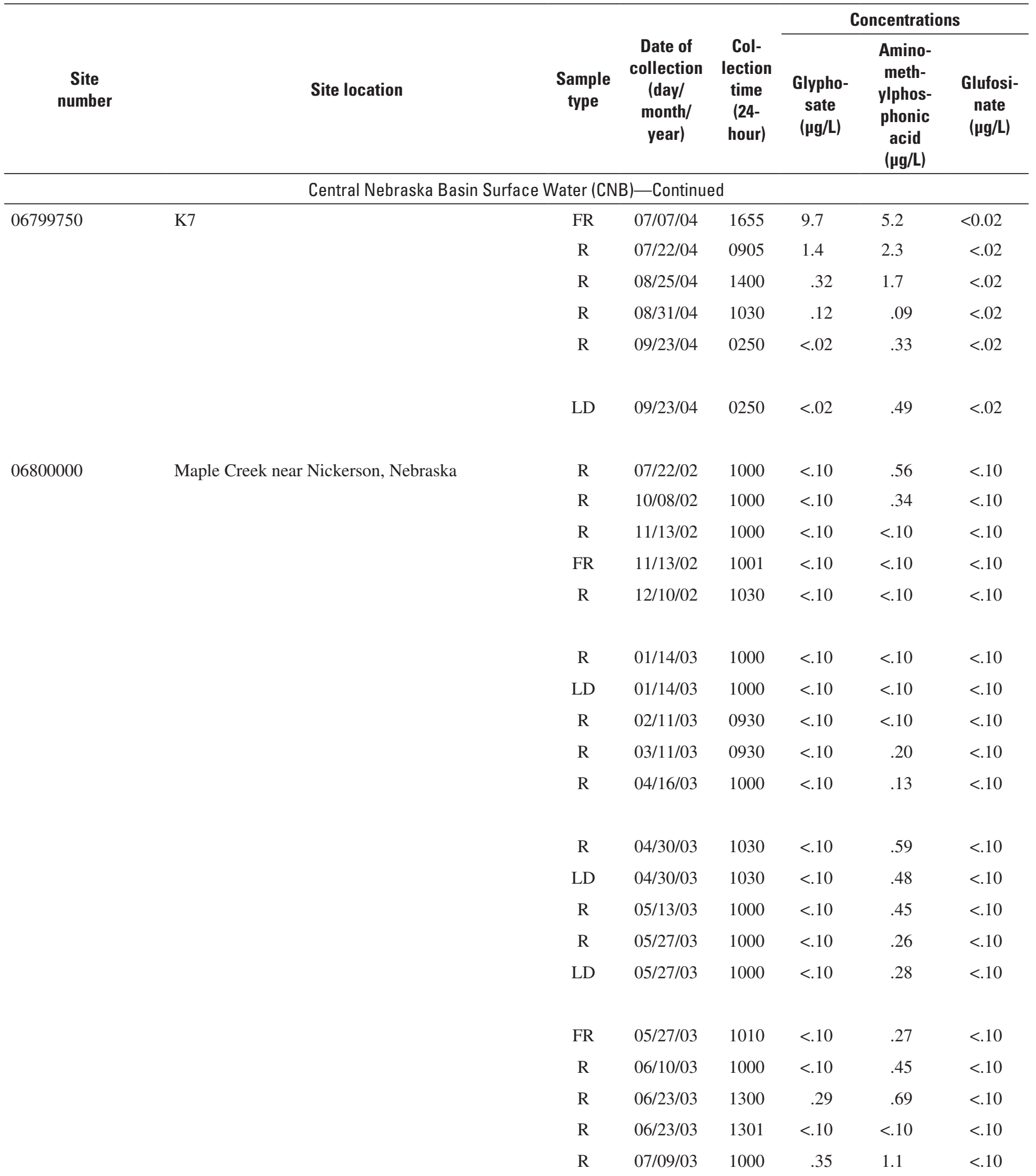


Table 5. Analytical results for glyphosate, aminomethylphosphonic acid, and glufosinate in National Water-Quality Assessment (NAWQA) Program surface- and ground-water studies, 2001-06.-Continued

[Location of NAWQA study units shown in figure 4. Sample type: FB, field blank; FR, field replicate; R, regular; LD, laboratory duplicate; --, no data; <, less than; $\mu \mathrm{g} / \mathrm{L}$, micrograms per liter]

\begin{tabular}{|c|c|c|c|c|c|c|c|}
\hline \multirow[b]{2}{*}{$\begin{array}{c}\text { Site } \\
\text { number }\end{array}$} & \multirow[b]{2}{*}{ Site location } & \multirow[b]{2}{*}{$\begin{array}{c}\text { Sample } \\
\text { type }\end{array}$} & \multirow[b]{2}{*}{$\begin{array}{c}\text { Date of } \\
\text { collection } \\
\text { (day/ } \\
\text { month/ } \\
\text { year) }\end{array}$} & \multirow[b]{2}{*}{$\begin{array}{c}\text { Col- } \\
\text { lection } \\
\text { time } \\
(24- \\
\text { hour) }\end{array}$} & \multicolumn{3}{|c|}{ Concentrations } \\
\hline & & & & & $\begin{array}{c}\text { Glypho- } \\
\text { sate } \\
(\mu \mathrm{g} / \mathrm{L})\end{array}$ & $\begin{array}{c}\text { Amino- } \\
\text { meth- } \\
\text { ylphos- } \\
\text { phonic } \\
\text { acid } \\
(\mu \mathrm{g} / \mathrm{L})\end{array}$ & $\begin{array}{c}\text { Glufosi- } \\
\text { nate } \\
\text { ( } \mu \mathrm{g} / \mathrm{L})\end{array}$ \\
\hline \multicolumn{8}{|c|}{ Central Nebraska Basin Surface Water (CNB)—Continued } \\
\hline \multirow[t]{27}{*}{06800000} & Maple Creek near Nickerson, Nebraska_- & $\mathrm{R}$ & 08/04/03 & 1000 & $<0.10$ & 0.50 & $<0.10$ \\
\hline & & FR & 08/04/03 & 1010 & $<.10$ & .46 & $<.10$ \\
\hline & & $\mathrm{R}$ & $08 / 19 / 03$ & 1100 & $<.10$ & .27 & $<.10$ \\
\hline & & $\mathrm{R}$ & 09/02/03 & 1100 & $<.10$ & .12 & $<.10$ \\
\hline & & $\mathrm{R}$ & 09/16/03 & 0930 & .16 & .35 & $<.10$ \\
\hline & & $\mathrm{LD}$ & $09 / 16 / 03$ & 0930 & .15 & .43 & $<.10$ \\
\hline & & $\mathrm{R}$ & $03 / 31 / 04$ & 1030 & .16 & .29 & $<.10$ \\
\hline & & $\mathrm{R}$ & $04 / 07 / 04$ & 1100 & $<.10$ & 1.4 & $<.10$ \\
\hline & & LD & 04/07/04 & 1100 & $<.10$ & 1.4 & $<.10$ \\
\hline & & $\mathrm{R}$ & $04 / 12 / 04$ & 1000 & .06 & .15 & .15 \\
\hline & & FR & $04 / 12 / 04$ & 1007 & .05 & .16 & .16 \\
\hline & & $\mathrm{R}$ & 04/19/04 & 1000 & $<.02$ & 1.6 & $<.02$ \\
\hline & & FR & 04/19/04 & 1007 & $<.02$ & 1.5 & $<.02$ \\
\hline & & $\mathrm{R}$ & 04/27/04 & 1000 & .11 & .62 & .11 \\
\hline & & $\mathrm{LD}$ & $04 / 27 / 04$ & 1000 & .15 & .62 & .12 \\
\hline & & $\mathrm{R}$ & 05/03/04 & 1030 & .08 & .19 & .12 \\
\hline & & LD & 05/03/04 & 1030 & .07 & .19 & .11 \\
\hline & & $\mathrm{R}$ & 05/13/04 & 1030 & .20 & .56 & .12 \\
\hline & & $\mathrm{LD}$ & 05/13/04 & 1030 & .26 & .56 & .13 \\
\hline & & $\mathrm{R}$ & 05/18/04 & 0930 & .06 & .63 & $<.02$ \\
\hline & & $\mathrm{LD}$ & 05/18/04 & 0930 & $<.02$ & .63 & $<.02$ \\
\hline & & $\mathrm{R}$ & 05/25/04 & 1030 & .09 & .58 & $<.02$ \\
\hline & & LD & 05/25/04 & 1030 & .09 & .59 & $<.02$ \\
\hline & & $\mathrm{R}$ & 06/01/04 & 1100 & .28 & .38 & $<.02$ \\
\hline & & LD & 06/01/04 & 1100 & .27 & .82 & $<.02$ \\
\hline & & $\mathrm{R}$ & 06/08/04 & 1030 & .20 & .70 & $<.02$ \\
\hline & & $\mathrm{R}$ & 06/15/04 & 1030 & .26 & .64 & $<.02$ \\
\hline
\end{tabular}


Table 5. Analytical results for glyphosate, aminomethylphosphonic acid, and glufosinate in National Water-Quality Assessment (NAWQA) Program surface- and ground-water studies, 2001-06.-Continued

[Location of NAWQA study units shown in figure 4. Sample type: FB, field blank; FR, field replicate; R, regular; LD, laboratory duplicate; --, no data; <, less than; $\mu \mathrm{g} / \mathrm{L}$, micrograms per liter]

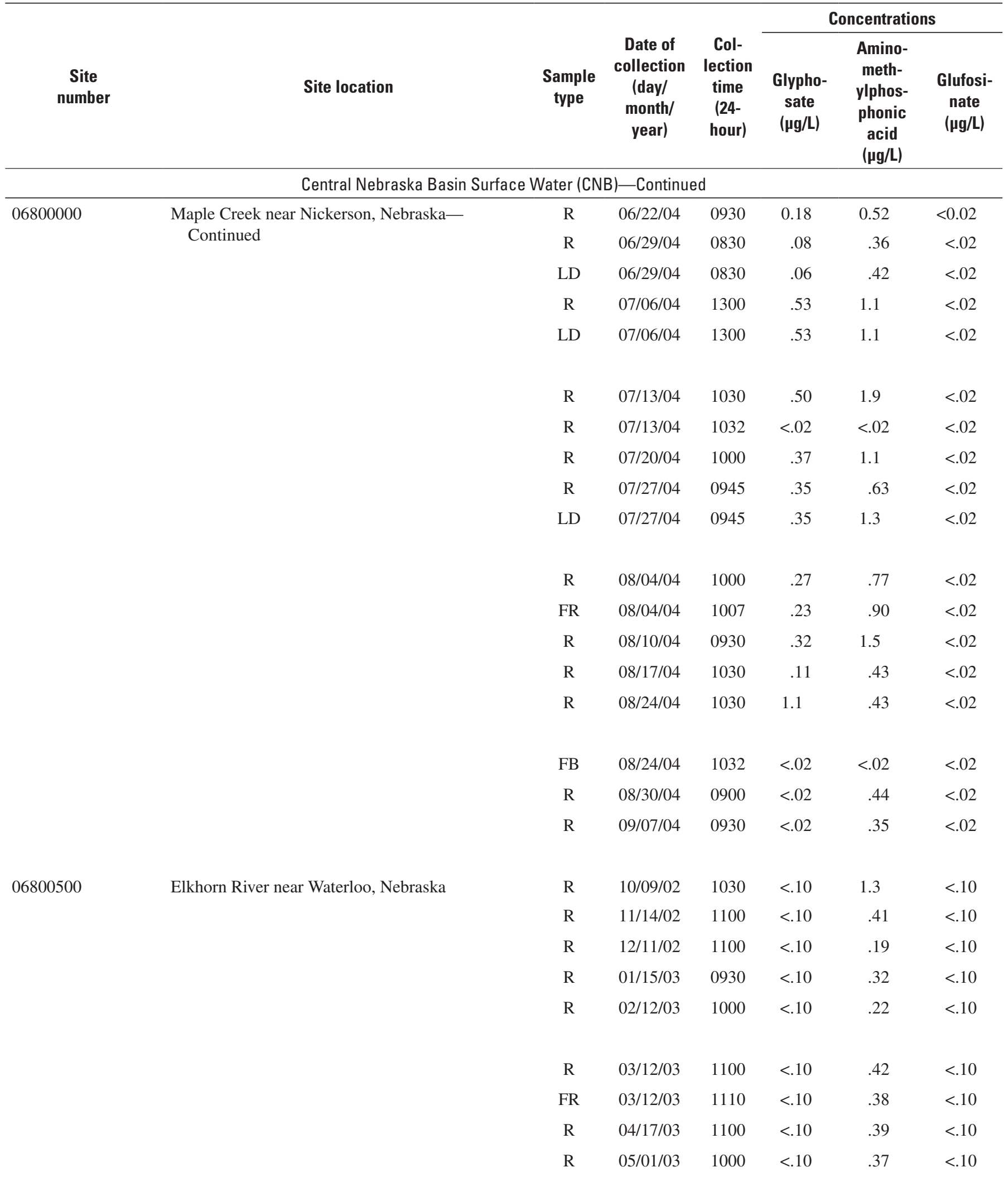


Table 5. Analytical results for glyphosate, aminomethylphosphonic acid, and glufosinate in National Water-Quality Assessment (NAWQA) Program surface- and ground-water studies, 2001-06.-Continued

[Location of NAWQA study units shown in figure 4. Sample type: FB, field blank; FR, field replicate; R, regular; LD, laboratory duplicate; --, no data; <, less than; $\mu \mathrm{g} / \mathrm{L}$, micrograms per liter]

\begin{tabular}{|c|c|c|c|c|c|c|c|}
\hline \multirow[b]{2}{*}{$\begin{array}{c}\text { Site } \\
\text { number }\end{array}$} & \multirow[b]{2}{*}{ Site location } & \multirow[b]{2}{*}{$\begin{array}{l}\text { Sample } \\
\text { type }\end{array}$} & \multirow[b]{2}{*}{$\begin{array}{c}\text { Date of } \\
\text { collection } \\
\text { (day/ } \\
\text { month/ } \\
\text { year) }\end{array}$} & \multirow[b]{2}{*}{$\begin{array}{c}\text { Col- } \\
\text { lection } \\
\text { time } \\
\text { (24- } \\
\text { hour) }\end{array}$} & \multicolumn{3}{|c|}{ Concentrations } \\
\hline & & & & & $\begin{array}{c}\text { Glypho- } \\
\text { sate } \\
\text { ( } \mu \mathrm{g} / \mathrm{L})\end{array}$ & $\begin{array}{c}\text { Amino- } \\
\text { meth- } \\
\text { ylphos- } \\
\text { phonic } \\
\text { acid } \\
\text { ( } \mu \mathrm{g} / \mathrm{L})\end{array}$ & $\begin{array}{c}\text { Glufosi- } \\
\text { nate } \\
\text { ( } \mathrm{\mu g} / \mathrm{L})\end{array}$ \\
\hline \multirow[t]{11}{*}{06800500} & \multirow{11}{*}{$\begin{array}{l}\text { Elkhorn River near Waterloo, Nebraska- } \\
\text { Continued }\end{array}$} & $\mathrm{R}$ & $05 / 14 / 03$ & 1000 & $<0.10$ & 0.21 & $<0.10$ \\
\hline & & $\mathrm{R}$ & $05 / 28 / 03$ & 1000 & $<.10$ & .34 & $<.10$ \\
\hline & & FR & $05 / 28 / 03$ & 1010 & $<.10$ & .50 & $<.10$ \\
\hline & & $\mathrm{R}$ & $06 / 11 / 03$ & 0930 & $<.10$ & .45 & $<.10$ \\
\hline & & $\mathrm{R}$ & 07/07/03 & 1401 & $<.10$ & $<.10$ & $<.10$ \\
\hline & & $\mathrm{R}$ & 07/10/03 & 0930 & .3 & .84 & $<.10$ \\
\hline & & $\mathrm{R}$ & $07 / 23 / 03$ & 1000 & $<.10$ & .58 & $<.10$ \\
\hline & & $\mathrm{R}$ & 08/06/03 & 1030 & $<.10$ & .60 & $<.10$ \\
\hline & & $\mathrm{R}$ & 08/20/03 & 1000 & $<.10$ & 1.4 & $<.10$ \\
\hline & & $\mathrm{R}$ & 09/03/03 & 1200 & $<.10$ & 1.1 & $<.10$ \\
\hline & & $\mathrm{R}$ & 09/17/03 & 1000 & .11 & .92 & $<.10$ \\
\hline \multirow{4}{*}{413302097090530} & \multirow{4}{*}{$\begin{array}{l}\text { Vadose zone ACT VZ1-1A near Schuyler, } \\
\text { Nebraska }\end{array}$} & $\mathrm{R}$ & $06 / 22 / 04$ & 1020 & .21 & .62 & $<.02$ \\
\hline & & $\mathrm{R}$ & 07/06/04 & 1035 & $<.02$ & $<.02$ & $<.02$ \\
\hline & & $\mathrm{R}$ & $07 / 22 / 04$ & 0925 & $<.02$ & $<.02$ & $<.02$ \\
\hline & & $\mathrm{R}$ & 07/23/04 & 0900 & .10 & .31 & $<.02$ \\
\hline 413302097090533 & $\begin{array}{l}\text { Vadose zone ACT VZ1-1D near Schuyler, } \\
\text { Nebraska }\end{array}$ & $\mathrm{R}$ & $04 / 02 / 04$ & 1040 & $<.02$ & $<.02$ & $<.02$ \\
\hline \multirow[t]{4}{*}{413334096325330} & \multirow{4}{*}{$\begin{array}{l}\text { Vadose zone ACT VZ1-2A near Nickerson, } \\
\text { Nebraska }\end{array}$} & $\mathrm{R}$ & 05/11/04 & 1400 & .02 & .14 & .31 \\
\hline & & $\mathrm{R}$ & $05 / 14 / 04$ & 1030 & .03 & .18 & $<.02$ \\
\hline & & $\mathrm{R}$ & 05/18/04 & 1605 & .03 & .08 & $<.02$ \\
\hline & & $\mathrm{R}$ & $05 / 24 / 04$ & 1235 & .02 & .09 & $<.02$ \\
\hline
\end{tabular}


Table 5. Analytical results for glyphosate, aminomethylphosphonic acid, and glufosinate in National Water-Quality Assessment (NAWQA) Program surface- and ground-water studies, 2001-06.-Continued

[Location of NAWQA study units shown in figure 4. Sample type: FB, field blank; FR, field replicate; R, regular; LD, laboratory duplicate; --, no data; <, less than; $\mu \mathrm{g} / \mathrm{L}$, micrograms per liter]

\begin{tabular}{|c|c|c|c|c|c|c|c|}
\hline \multirow[b]{2}{*}{$\begin{array}{c}\text { Site } \\
\text { number }\end{array}$} & \multirow[b]{2}{*}{ Site location } & \multirow[b]{2}{*}{$\begin{array}{l}\text { Sample } \\
\text { type }\end{array}$} & \multirow[b]{2}{*}{$\begin{array}{c}\text { Date of } \\
\text { collection } \\
\text { (day/ } \\
\text { month/ } \\
\text { year) }\end{array}$} & \multirow[b]{2}{*}{$\begin{array}{c}\text { Col- } \\
\text { lection } \\
\text { time } \\
\text { (24- } \\
\text { hour) }\end{array}$} & \multicolumn{3}{|c|}{ Concentrations } \\
\hline & & & & & $\begin{array}{c}\text { Glypho- } \\
\text { sate } \\
\text { (pg/L) }\end{array}$ & $\begin{array}{c}\text { Amino- } \\
\text { meth- } \\
\text { ylphos- } \\
\text { phonic } \\
\text { acid } \\
\text { ( } \mu \mathrm{g} / \mathrm{L})\end{array}$ & $\begin{array}{c}\text { Glufosi- } \\
\text { nate } \\
\text { ( } \mu \mathrm{g} / \mathrm{L})\end{array}$ \\
\hline 413334096325330 & $\begin{array}{l}\text { Vadose zone ACT VZ1-2A near Nickerson, } \\
\text { Nebraska }\end{array}$ & $\mathrm{R}$ & 07/06/04 & 1240 & $<0.02$ & $<0.02$ & $<0.02$ \\
\hline \multirow[t]{2}{*}{413334096325331} & \multirow{2}{*}{$\begin{array}{l}\text { Vadose zone ACT VZ1-2B near Nickerson, } \\
\text { Nebraska }\end{array}$} & $\mathrm{R}$ & 03/30/04 & 1200 & $<.02$ & $<.02$ & $<.02$ \\
\hline & & $\mathrm{R}$ & $04 / 12 / 05$ & 1518 & $<.02$ & .02 & $<.02$ \\
\hline \multirow[t]{2}{*}{413334096325332} & \multirow{2}{*}{$\begin{array}{l}\text { Valdose zone ACT VZ1-2C near Nickerson, } \\
\text { Nebraska }\end{array}$} & $\mathrm{R}$ & $04 / 15 / 04$ & 1035 & $<.02$ & $<.02$ & $<.02$ \\
\hline & & FR & $04 / 15 / 04$ & 1040 & $<.02$ & $<.02$ & $<.02$ \\
\hline \multirow[t]{2}{*}{413341096325530} & \multirow{2}{*}{$\begin{array}{l}\text { Vadose zone ACT VZ1-3A near Nickerson, } \\
\text { Nebraska }\end{array}$} & $\mathrm{R}$ & $06 / 14 / 04$ & 1200 & $<.02$ & .29 & $<.02$ \\
\hline & & $\mathrm{R}$ & 07/06/04 & 1255 & $<.02$ & $<.02$ & $<.02$ \\
\hline \multirow[t]{3}{*}{413341096325531} & \multirow{3}{*}{$\begin{array}{l}\text { Vadose zone ACT VZ1-3B near Nickerson, } \\
\text { Nebraska }\end{array}$} & $\mathrm{R}$ & 05/11/04 & 1430 & .04 & .14 & .29 \\
\hline & & $\mathrm{R}$ & 08/19/04 & 1150 & $<.02$ & .06 & $<.26$ \\
\hline & & $\mathrm{R}$ & $04 / 12 / 05$ & 1540 & $<.02$ & .46 & $<.02$ \\
\hline \multirow[t]{3}{*}{413350096324730} & \multirow{3}{*}{$\begin{array}{l}\text { Vadose zone ACT VZ1-4A near Nickerson, } \\
\text { Nebraska }\end{array}$} & $\mathrm{R}$ & 05/11/04 & 1345 & .02 & .08 & .26 \\
\hline & & $\mathrm{R}$ & $05 / 24 / 04$ & 1250 & $<.02$ & .04 & $<.02$ \\
\hline & & $\mathrm{R}$ & 06/14/04 & 1120 & .08 & .33 & $<.02$ \\
\hline \multirow[t]{4}{*}{413350096324731} & \multirow{4}{*}{$\begin{array}{l}\text { Vadose zone ACT VZ1-4B near Nickerson, } \\
\text { Nebraska }\end{array}$} & $\mathrm{R}$ & $05 / 24 / 04$ & 1255 & .02 & .06 & $<.02$ \\
\hline & & $\mathrm{R}$ & 06/14/04 & 1125 & .06 & .20 & $<.02$ \\
\hline & & $\mathrm{R}$ & 08/19/04 & 1405 & $<.02$ & .04 & $<.02$ \\
\hline & & $\mathrm{R}$ & 07/06/04 & 1225 & .67 & .21 & $<.02$ \\
\hline
\end{tabular}


Table 5. Analytical results for glyphosate, aminomethylphosphonic acid, and glufosinate in National Water-Quality Assessment (NAWQA) Program surface- and ground-water studies, 2001-06.-Continued

[Location of NAWQA study units shown in figure 4. Sample type: FB, field blank; FR, field replicate; R, regular; LD, laboratory duplicate; --, no data; <, less than; $\mu \mathrm{g} / \mathrm{L}$, micrograms per liter]

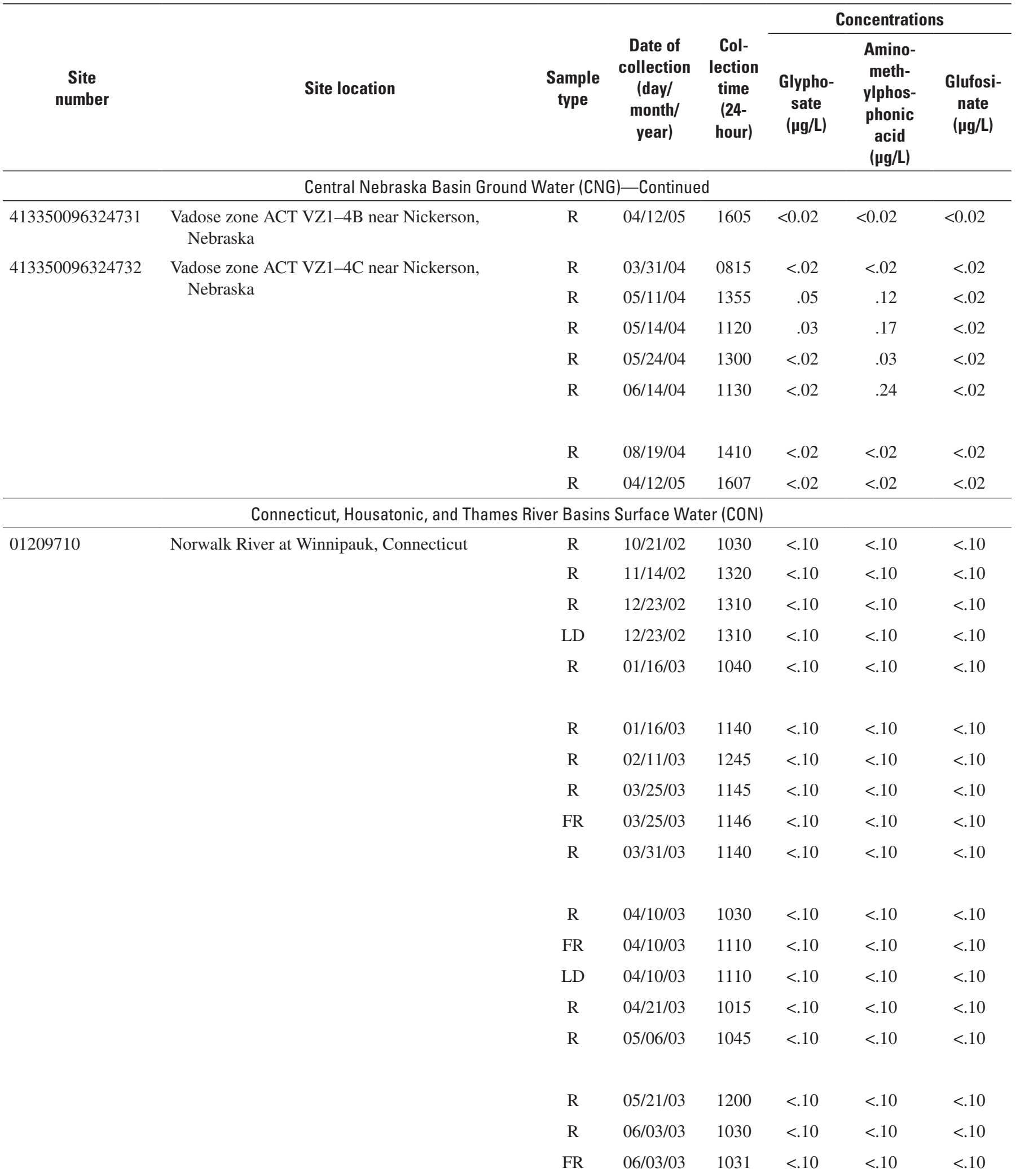


Table 5. Analytical results for glyphosate, aminomethylphosphonic acid, and glufosinate in National Water-Quality Assessment (NAWQA) Program surface- and ground-water studies, 2001-06.-Continued

[Location of NAWQA study units shown in figure 4. Sample type: FB, field blank; FR, field replicate; R, regular; LD, laboratory duplicate; --, no data; <, less than; $\mu \mathrm{g} / \mathrm{L}$, micrograms per liter]

\begin{tabular}{|c|c|c|c|c|c|c|c|}
\hline \multirow[b]{2}{*}{$\begin{array}{c}\text { Site } \\
\text { number }\end{array}$} & \multirow[b]{2}{*}{ Site location } & \multirow[b]{2}{*}{$\begin{array}{l}\text { Sample } \\
\text { type }\end{array}$} & \multirow[b]{2}{*}{$\begin{array}{c}\text { Date of } \\
\text { collection } \\
\text { (day/ } \\
\text { month/ } \\
\text { year) }\end{array}$} & \multirow[b]{2}{*}{$\begin{array}{c}\text { Col- } \\
\text { lection } \\
\text { time } \\
\text { (24- } \\
\text { hour) }\end{array}$} & \multicolumn{3}{|c|}{ Concentrations } \\
\hline & & & & & $\begin{array}{c}\text { Glypho- } \\
\text { sate } \\
\text { ( } \mu \mathrm{g} / \mathrm{L})\end{array}$ & $\begin{array}{c}\text { Amino- } \\
\text { meth- } \\
\text { ylphos- } \\
\text { phonic } \\
\text { acid } \\
\text { ( } \mu \mathrm{g} / \mathrm{L})\end{array}$ & $\begin{array}{c}\text { Glufosi- } \\
\text { nate } \\
\text { ( } \mu \mathrm{g} / \mathrm{L})\end{array}$ \\
\hline \multirow[t]{6}{*}{01209710} & \multirow{2}{*}{$\begin{array}{l}\text { Norwalk River at Winnipauk, Connecticut- } \\
\text { Continued }\end{array}$} & $\mathrm{R}$ & 07/08/03 & 1030 & $<0.10$ & $<0.10$ & $<0.10$ \\
\hline & & LD & $07 / 08 / 03$ & 1030 & $<.10$ & $<.10$ & $<.10$ \\
\hline & & $\mathrm{R}$ & $06 / 24 / 03$ & 0930 & $<.10$ & $<.10$ & $<.10$ \\
\hline & & FR & $06 / 24 / 03$ & 1000 & $<.10$ & $<.10$ & $<.10$ \\
\hline & & $\mathrm{R}$ & 08/14/03 & 0950 & $<.10$ & .12 & $<.10$ \\
\hline & & $\mathrm{R}$ & 09/03/03 & 1130 & $<.10$ & .12 & $<.10$ \\
\hline \multicolumn{8}{|c|}{ Delmarva Peninsula Ground Water (JMD) } \\
\hline 380027075410802 & SOD344 & $\mathrm{R}$ & 08/02/01 & 0930 & $<0.10$ & $<0.10$ & $<0.10$ \\
\hline 380358075292901 & WO Fe 1 & $\mathrm{R}$ & $01 / 15 / 03$ & 1100 & $<.10$ & $<.10$ & $<.10$ \\
\hline \multirow[t]{3}{*}{380403075292901} & WOFc46 & FB & 09/06/01 & 1000 & $<.10$ & $<.10$ & $<.10$ \\
\hline & & $\mathrm{R}$ & 09/06/01 & 0950 & $<.10$ & $<.10$ & $<.10$ \\
\hline & & FR & 09/06/01 & 1030 & $<.10$ & $<.10$ & $<.10$ \\
\hline 381543075273802 & WOCc3 & $\mathrm{R}$ & 08/08/01 & 1430 & $<.10$ & $<.10$ & $<.10$ \\
\hline 381754075083601 & WOCg76 & $\mathrm{R}$ & 08/09/01 & 0900 & $<.10$ & $<.10$ & $<.10$ \\
\hline 381754075083603 & WOCg78 & $\mathrm{R}$ & 08/09/01 & 1300 & $<.10$ & $<.10$ & $<.10$ \\
\hline \multirow[t]{2}{*}{382403075233201} & WICh50 & $\mathrm{R}$ & 09/24/01 & 1000 & $<.10$ & $<.10$ & $<.10$ \\
\hline & & FR & 09/24/01 & 1030 & $<.10$ & $<.10$ & $<.10$ \\
\hline 382824075081502 & Ri22-10 & $\mathrm{R}$ & $01 / 14 / 03$ & 1400 & $<.10$ & $<.10$ & $<.10$ \\
\hline 382825075081601 & Ri22-03 & $\mathrm{R}$ & 09/05/01 & 1100 & $<.10$ & $<.10$ & $<.10$ \\
\hline \multirow[t]{2}{*}{382833075213701} & Rf24-08 & $\mathrm{R}$ & 01/16/03 & 1400 & $<.10$ & $<.10$ & $<.10$ \\
\hline & & LD & $01 / 16 / 03$ & 1400 & $<.10$ & $<.10$ & $<.10$ \\
\hline 382927075211701 & Rf14-02 & $\mathrm{R}$ & 10/11/01 & 1000 & $<.10$ & $<.10$ & $<.10$ \\
\hline
\end{tabular}


Table 5. Analytical results for glyphosate, aminomethylphosphonic acid, and glufosinate in National Water-Quality Assessment (NAWQA) Program surface- and ground-water studies, 2001-06.-Continued

[Location of NAWQA study units shown in figure 4. Sample type: FB, field blank; FR, field replicate; R, regular; LD, laboratory duplicate; --, no data; <, less than; $\mu \mathrm{g} / \mathrm{L}$, micrograms per liter]

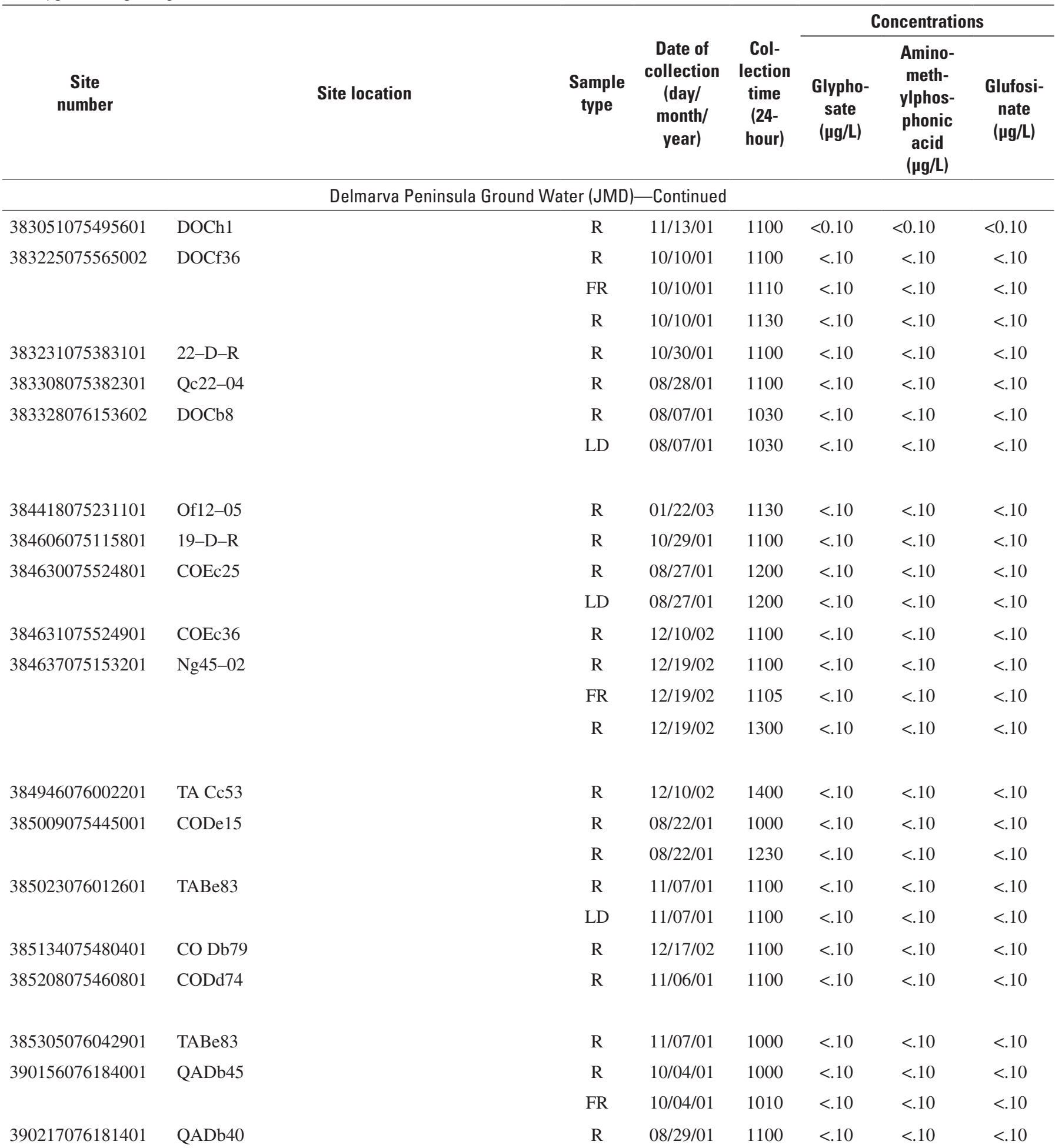


Table 5. Analytical results for glyphosate, aminomethylphosphonic acid, and glufosinate in National Water-Quality Assessment (NAWQA) Program surface- and ground-water studies, 2001-06.-Continued

[Location of NAWQA study units shown in figure 4. Sample type: FB, field blank; FR, field replicate; R, regular; LD, laboratory duplicate; --, no data; <, less than; $\mu \mathrm{g} / \mathrm{L}$, micrograms per liter]

\begin{tabular}{|c|c|c|c|c|c|c|c|}
\hline \multirow[b]{2}{*}{$\begin{array}{c}\text { Site } \\
\text { number }\end{array}$} & \multirow[b]{2}{*}{ Site location } & \multirow[b]{2}{*}{$\begin{array}{c}\text { Sample } \\
\text { type }\end{array}$} & \multirow[b]{2}{*}{$\begin{array}{c}\text { Date of } \\
\text { collection } \\
\text { (day/ } \\
\text { month/ } \\
\text { year) }\end{array}$} & \multirow[b]{2}{*}{$\begin{array}{l}\text { Col- } \\
\text { lection } \\
\text { time } \\
(24- \\
\text { hour) }\end{array}$} & \multicolumn{3}{|c|}{ Concentrations } \\
\hline & & & & & $\begin{array}{c}\text { Glypho- } \\
\text { sate } \\
(\mu \mathrm{g} / \mathrm{L})\end{array}$ & $\begin{array}{c}\text { Amino- } \\
\text { meth- } \\
\text { ylphos- } \\
\text { phonic } \\
\text { acid } \\
(\mu \mathrm{g} / \mathrm{L})\end{array}$ & $\begin{array}{c}\text { Glufosi- } \\
\text { nate } \\
\text { ( } \mu \text { g/L) }\end{array}$ \\
\hline \multicolumn{8}{|c|}{ Delmarva Peninsula Ground Water (JMD)—Continued } \\
\hline 390217076181401 & QADb40 & $\mathrm{LD}$ & 08/29/01 & 1100 & $<0.10$ & $<0.10$ & $<0.10$ \\
\hline \multirow[t]{3}{*}{390918075291701} & Equipment blank & $\mathrm{R}$ & $11 / 29 / 01$ & 1300 & $<.10$ & $<.10$ & $<.10$ \\
\hline & & $\mathrm{LD}$ & $11 / 29 / 01$ & 1330 & $<.10$ & $<.10$ & $<.10$ \\
\hline & & $\mathrm{R}$ & $12 / 09 / 02$ & 1530 & $<.10$ & $<.10$ & $<.10$ \\
\hline 391240075432001 & Ib32-08 & $\mathrm{R}$ & $12 / 17 / 02$ & 1500 & $<.10$ & $<.10$ & $<.10$ \\
\hline 392057075443601 & Gb51-07 & $\mathrm{R}$ & $01 / 28 / 03$ & 1100 & $<.10$ & $<.10$ & $<.10$ \\
\hline \multirow[t]{3}{*}{392403075362101} & GC14-04 & $\mathrm{R}$ & $12 / 09 / 02$ & 1200 & $<.10$ & $<.10$ & $<.10$ \\
\hline & & $\mathrm{LD}$ & $12 / 09 / 02$ & 1200 & $<.10$ & $<.10$ & $<.10$ \\
\hline & & FR & $12 / 09 / 02$ & 1205 & $<.10$ & $<.10$ & $<.10$ \\
\hline 392414075361001 & Ib32-05 DLMV NAWQA & $\mathrm{R}$ & 08/23/01 & 0930 & $<.10$ & $<.10$ & $<.10$ \\
\hline 394224075340501 & Cd31-19 DLMV NAWQA & $\mathrm{R}$ & 08/06/01 & 1000 & $<.10$ & $<.10$ & $<.10$ \\
\hline \multicolumn{8}{|c|}{ Eastern lowa Basin Surface Water (SKI) } \\
\hline \multirow[t]{2}{*}{05420500} & Mississippi River at Clinton, Iowa & $\mathrm{R}$ & 05/09/01 & 1040 & $<.10$ & $<.10$ & $<.10$ \\
\hline & & $\mathrm{LD}$ & 05/09/01 & 1040 & $<.10$ & $<.10$ & $<.10$ \\
\hline 05448095 & Mississippi River at Buffalo, Iowa & $\mathrm{R}$ & $04 / 25 / 01$ & 0915 & $<.10$ & $<.10$ & $<.10$ \\
\hline 05466000 & Mississippi River at Keith, Iowa & $\mathrm{R}$ & $04 / 26 / 01$ & 1730 & $<.10$ & $<.10$ & $<.10$ \\
\hline 05492200 & Mississippi River at Alexandria, Missouri & $\mathrm{R}$ & $04 / 26 / 01$ & 1215 & $<.10$ & .12 & $<.10$ \\
\hline 05587455 & Mississippi River below Grafton, Illinois & $\mathrm{R}$ & $05 / 14 / 01$ & 1445 & $<.10$ & $<.10$ & $<.10$ \\
\hline \multicolumn{8}{|c|}{ Eastern lowa Basin Ground Water (SKI) } \\
\hline 421850093092501 & USGS Monitoring Well FS1-11 & $\mathrm{R}$ & $12 / 20 / 05$ & 1700 & $<.02$ & $<.02$ & $<.02$ \\
\hline 421853093091601 & USGS Monitoring Well FS1-3A & $\mathrm{R}$ & $12 / 21 / 05$ & 1330 & $<.02$ & $<.02$ & $<.02$ \\
\hline 421853093091603 & USGS Monitoring Well FS1-3C & $\mathrm{R}$ & $12 / 21 / 05$ & 1600 & $<.02$ & $<.02$ & $<.02$ \\
\hline \multirow[t]{2}{*}{421854093091501} & USGS Monitoring Well FS1-4A & $\mathrm{R}$ & $12 / 22 / 05$ & 1615 & $<.02$ & $<.02$ & $<.02$ \\
\hline & & $\mathrm{LD}$ & $12 / 22 / 05$ & 1615 & $<.02$ & $<.02$ & $<.02$ \\
\hline \multirow[t]{2}{*}{421854093091502} & USGS Monitoring Well FS1-4B & $\mathrm{R}$ & $12 / 22 / 05$ & 1415 & $<.02$ & $<.02$ & $<.02$ \\
\hline & & FR & $12 / 22 / 05$ & 1420 & $<.02$ & $<.02$ & $<.02$ \\
\hline 421854093091503 & USGS Monitoring Well FS1-4C & $\mathrm{R}$ & $12 / 22 / 05$ & 1315 & $<.02$ & $<.02$ & $<.02$ \\
\hline 421855093091601 & USGS Monitoring Well FS1-5A & $\mathrm{R}$ & $12 / 29 / 05$ & 1745 & $<.02$ & $<.02$ & $<.02$ \\
\hline 421855093091602 & USGS Monitoring Well FS1-5B & $\mathrm{R}$ & $12 / 30 / 05$ & 1200 & $<.02$ & $<.02$ & $<.02$ \\
\hline
\end{tabular}


Table 5. Analytical results for glyphosate, aminomethylphosphonic acid, and glufosinate in National Water-Quality Assessment (NAWQA) Program surface- and ground-water studies, 2001-06.-Continued

[Location of NAWQA study units shown in figure 4. Sample type: FB, field blank; FR, field replicate; R, regular; LD, laboratory duplicate; --, no data; <, less than; $\mu \mathrm{g} / \mathrm{L}$, micrograms per liter]

\begin{tabular}{|c|c|c|c|c|c|c|c|}
\hline \multirow[b]{2}{*}{$\begin{array}{c}\text { Site } \\
\text { number }\end{array}$} & \multirow[b]{2}{*}{ Site location } & \multirow[b]{2}{*}{$\begin{array}{l}\text { Sample } \\
\text { type }\end{array}$} & \multirow[b]{2}{*}{$\begin{array}{c}\text { Date of } \\
\text { collection } \\
\text { (day/ } \\
\text { month/ } \\
\text { year) }\end{array}$} & \multirow[b]{2}{*}{$\begin{array}{c}\text { Col- } \\
\text { lection } \\
\text { time } \\
(24- \\
\text { hour) }\end{array}$} & \multicolumn{3}{|c|}{ Concentrations } \\
\hline & & & & & $\begin{array}{c}\text { Glypho- } \\
\text { sate } \\
(\mu \mathrm{g} / \mathrm{L})\end{array}$ & $\begin{array}{c}\text { Amino- } \\
\text { meth- } \\
\text { ylphos- } \\
\text { phonic } \\
\text { acid } \\
(\mu \mathrm{g} / \mathrm{L})\end{array}$ & $\begin{array}{c}\text { Glufosi- } \\
\text { nate } \\
(\mu \mathrm{g} / \mathrm{L})\end{array}$ \\
\hline \multicolumn{8}{|c|}{ Eastern lowa Basin Ground Water (SKI)—Continued } \\
\hline 421856093091501 & USGS Monitoring Well TR1-1A & $\mathrm{R}$ & $12 / 23 / 05$ & 1145 & $<0.02$ & $<0.02$ & $<0.02$ \\
\hline 421856093091502 & USGS Monitoring Well TR1-2B & $\mathrm{R}$ & $12 / 29 / 05$ & 1145 & $<.02$ & $<.02$ & $<.02$ \\
\hline \multicolumn{8}{|c|}{ Georgia-Florida Coastal Plain Surface Water (GFL) } \\
\hline \multirow[t]{22}{*}{02317797} & \multirow{22}{*}{$\begin{array}{l}\text { Little River at Upper Ty Ty Road near Tifton, } \\
\text { Georgia }\end{array}$} & $\mathrm{R}$ & $11 / 04 / 02$ & 1250 & $<.10$ & $<.10$ & $<.10$ \\
\hline & & FR & $11 / 04 / 02$ & 1300 & $<.10$ & $<.10$ & $<.10$ \\
\hline & & $\mathrm{R}$ & $12 / 03 / 02$ & 1300 & $<.10$ & $<.10$ & $<.10$ \\
\hline & & $\mathrm{R}$ & $02 / 04 / 03$ & 1200 & $<.10$ & $<.10$ & $<.10$ \\
\hline & & FR & $02 / 04 / 03$ & 1210 & $<.10$ & $<.10$ & $<.10$ \\
\hline & & $\mathrm{R}$ & 02/18/03 & 1140 & $<.10$ & $<.10$ & $<.10$ \\
\hline & & $\mathrm{LD}$ & $02 / 18 / 03$ & 1140 & $<.10$ & $<.10$ & $<.10$ \\
\hline & & $\mathrm{R}$ & $03 / 04 / 03$ & 1300 & $<.10$ & $<.10$ & $<.10$ \\
\hline & & $\mathrm{R}$ & 03/19/03 & 1210 & $<.10$ & $<.10$ & $<.10$ \\
\hline & & $\mathrm{R}$ & $03 / 31 / 03$ & 1240 & $<.10$ & $<.10$ & $<.10$ \\
\hline & & $\mathrm{R}$ & $04 / 14 / 03$ & 1220 & $<.10$ & $<.10$ & $<.10$ \\
\hline & & $\mathrm{R}$ & 05/06/03 & 1330 & $<.10$ & $<.10$ & $<.10$ \\
\hline & & $\mathrm{R}$ & $05 / 16 / 03$ & 1020 & $<.10$ & $<.10$ & $<.10$ \\
\hline & & FR & $05 / 16 / 03$ & 1030 & $<.10$ & $<.10$ & $<.10$ \\
\hline & & $\mathrm{R}$ & 06/02/03 & 1120 & $<.10$ & $<.10$ & $<.10$ \\
\hline & & $\mathrm{R}$ & 06/30/03 & 1130 & $<.10$ & $<.10$ & $<.10$ \\
\hline & & $\mathrm{R}$ & 08/05/03 & 1200 & $<.10$ & $<.10$ & $<.10$ \\
\hline & & $\mathrm{R}$ & 08/19/03 & 1230 & $<.10$ & $<.10$ & $<.10$ \\
\hline & & $\mathrm{R}$ & 09/02/03 & 1300 & $<.10$ & $<.10$ & $<.10$ \\
\hline & & $\mathrm{R}$ & $06 / 16 / 03$ & 1300 & $<.10$ & $<.10$ & $<.10$ \\
\hline & & $\mathrm{R}$ & $01 / 07 / 03$ & 1310 & $<.10$ & $<.10$ & $<.10$ \\
\hline & & $\mathrm{R}$ & $01 / 21 / 03$ & 1300 & $<.10$ & $<.10$ & $<.10$ \\
\hline \multirow[t]{2}{*}{02318500} & \multirow{2}{*}{$\begin{array}{l}\text { Withlacoochee River at U.S. } 84 \text { near Quitman, } \\
\text { Georgia }\end{array}$} & $\mathrm{R}$ & $10 / 01 / 02$ & 1130 & $<.10$ & .15 & $<.10$ \\
\hline & & $\mathrm{R}$ & $11 / 06 / 02$ & 1400 & $<.10$ & $<.10$ & $<.10$ \\
\hline
\end{tabular}


Table 5. Analytical results for glyphosate, aminomethylphosphonic acid, and glufosinate in National Water-Quality Assessment (NAWQA) Program surface- and ground-water studies, 2001-06.-Continued

[Location of NAWQA study units shown in figure 4. Sample type: FB, field blank; FR, field replicate; R, regular; LD, laboratory duplicate; --, no data; <, less than; $\mu \mathrm{g} / \mathrm{L}$, micrograms per liter]

\begin{tabular}{|c|c|c|c|c|c|c|c|}
\hline \multirow[b]{2}{*}{$\begin{array}{c}\text { Site } \\
\text { number }\end{array}$} & \multirow[b]{2}{*}{ Site location } & \multirow[b]{2}{*}{$\begin{array}{l}\text { Sample } \\
\text { type }\end{array}$} & \multirow[b]{2}{*}{$\begin{array}{c}\text { Date of } \\
\text { collection } \\
\text { (day/ } \\
\text { month/ } \\
\text { year) }\end{array}$} & \multirow[b]{2}{*}{$\begin{array}{c}\text { Col- } \\
\text { lection } \\
\text { time } \\
\text { (24- } \\
\text { hour) }\end{array}$} & \multicolumn{3}{|c|}{ Concentrations } \\
\hline & & & & & $\begin{array}{c}\text { Glypho- } \\
\text { sate } \\
(\mu \mathrm{g} / \mathrm{L})\end{array}$ & $\begin{array}{c}\text { Amino- } \\
\text { meth- } \\
\text { ylphos- } \\
\text { phonic } \\
\text { acid } \\
(\mu \mathrm{g} / \mathrm{L})\end{array}$ & $\begin{array}{c}\text { Glufosi- } \\
\text { nate } \\
(\mu \mathrm{g} / \mathrm{L})\end{array}$ \\
\hline \multicolumn{8}{|c|}{ Georgia-Florida Coastal Plain Surface Water (GFL)—Continued } \\
\hline \multirow[t]{4}{*}{02318500} & \multirow{4}{*}{$\begin{array}{l}\text { Withlacoochee River at U.S. } 84 \text { near Quitman, } \\
\text { Georgia }\end{array}$} & $\mathrm{R}$ & $12 / 04 / 02$ & 1100 & $<0.10$ & $<0.10$ & $<0.10$ \\
\hline & & $\mathrm{R}$ & 01/08/03 & 1310 & $<.10$ & $<.10$ & $<.10$ \\
\hline & & $\mathrm{R}$ & $02 / 03 / 03$ & 1340 & $<.10$ & $<.10$ & $<.10$ \\
\hline & & FR & 02/03/03 & 1330 & $<.10$ & $<.10$ & $<.10$ \\
\hline \multicolumn{8}{|c|}{ Great and Little Miami River Basins Surface Water (BET) } \\
\hline \multirow[t]{8}{*}{03267900} & Mad River at St. Paris Pike at Eagle City, Ohio & $\mathrm{R}$ & 09/19/01 & 1200 & $<.10$ & $<.10$ & $<.10$ \\
\hline & & FR & $09 / 19 / 01$ & 1208 & $<.10$ & $<.10$ & $<.10$ \\
\hline & & $\mathrm{R}$ & $12 / 05 / 01$ & 1100 & $<.10$ & .16 & $<.10$ \\
\hline & & $\mathrm{R}$ & $10 / 17 / 01$ & 1200 & $<.10$ & $<.10$ & $<.10$ \\
\hline & & $\mathrm{R}$ & $11 / 06 / 01$ & 0930 & $<.10$ & .11 & $<.10$ \\
\hline & & LD & $11 / 06 / 01$ & 0930 & $<.10$ & .14 & $<.10$ \\
\hline & & $\mathrm{R}$ & $01 / 09 / 02$ & 1030 & $<.10$ & .14 & $<.10$ \\
\hline & & LD & $01 / 09 / 02$ & 1030 & $<.10$ & .10 & $<.10$ \\
\hline 03274000 & Great Miami River at Hamilton, Ohio & $\mathrm{R}$ & 09/18/01 & 1100 & .1 & 1.2 & $<.10$ \\
\hline \multicolumn{8}{|c|}{ Great and Little Miami River Basins Ground Water (BET) } \\
\hline \multirow[t]{2}{*}{390744084212501} & URB-02 at Newton, Ohio & $\mathrm{R}$ & $11 / 29 / 01$ & 1100 & $<.10$ & $<.10$ & $<.10$ \\
\hline & & LD & $11 / 29 / 01$ & 1100 & $<.10$ & $<.10$ & $<.10$ \\
\hline 391226084272501 & URB-31 at Cincinnati, Ohio & $\mathrm{R}$ & $11 / 28 / 01$ & 1000 & $<.10$ & $<.10$ & $<.10$ \\
\hline \multirow[t]{3}{*}{391518084485201} & URB-04 at Harrison, Ohio & $\mathrm{R}$ & $11 / 15 / 01$ & 1400 & $<.10$ & $<.10$ & $<.10$ \\
\hline & & $\mathrm{R}$ & $11 / 15 / 01$ & 1000 & $<.10$ & $<.10$ & $<.10$ \\
\hline & & FR & $11 / 15 / 01$ & 1005 & $<.10$ & $<.10$ & $<.10$ \\
\hline 391849084381901 & URB-01 at Ross, Ohio & $\mathrm{R}$ & $11 / 27 / 01$ & 1400 & $<.10$ & $<.10$ & $<.10$ \\
\hline 392008084335501 & URB-06 at Fairfield, Ohio & $\mathrm{R}$ & $11 / 14 / 01$ & 1500 & $<.10$ & $<.10$ & $<.10$ \\
\hline 392008084343801 & URB-07 at Fairfield, Ohio & $\mathrm{R}$ & $11 / 26 / 01$ & 1500 & $<.10$ & $<.10$ & $<.10$ \\
\hline \multirow[t]{2}{*}{392047084325101} & URB-15 at Fairfield, Ohio & $\mathrm{R}$ & $11 / 14 / 01$ & 1100 & $<.10$ & $<.10$ & $<.10$ \\
\hline & & FR & $11 / 14 / 01$ & 1101 & $<.10$ & $<.10$ & $<.10$ \\
\hline 392049084340301 & URB-08 at Fairfield, Ohio & $\mathrm{R}$ & $11 / 27 / 01$ & 1000 & $<.10$ & $<.10$ & $<.10$ \\
\hline 392121084332101 & URB-29 at Hamilton, Ohio & $\mathrm{R}$ & $11 / 13 / 01$ & 1600 & $<.10$ & $<.10$ & $<.10$ \\
\hline 392917084205601 & URB-11 at Middletown, Ohio & $\mathrm{R}$ & $11 / 07 / 01$ & 1500 & $<.10$ & $<.10$ & $<.10$ \\
\hline
\end{tabular}


Table 5. Analytical results for glyphosate, aminomethylphosphonic acid, and glufosinate in National Water-Quality Assessment (NAWQA) Program surface- and ground-water studies, 2001-06.-Continued

[Location of NAWQA study units shown in figure 4. Sample type: FB, field blank; FR, field replicate; R, regular; LD, laboratory duplicate; --, no data; <, less than; $\mu \mathrm{g} / \mathrm{L}$, micrograms per liter]

\begin{tabular}{|c|c|c|c|c|c|c|c|}
\hline \multirow[b]{2}{*}{$\begin{array}{c}\text { Site } \\
\text { number }\end{array}$} & \multirow[b]{2}{*}{ Site location } & \multirow[b]{2}{*}{$\begin{array}{c}\text { Sample } \\
\text { type }\end{array}$} & \multirow[b]{2}{*}{$\begin{array}{c}\text { Date of } \\
\text { collection } \\
\text { (day/ } \\
\text { month/ } \\
\text { year) }\end{array}$} & \multirow[b]{2}{*}{$\begin{array}{c}\text { Col- } \\
\text { lection } \\
\text { time } \\
\text { (24- } \\
\text { hour) }\end{array}$} & \multicolumn{3}{|c|}{ Concentrations } \\
\hline & & & & & $\begin{array}{c}\text { Glypho- } \\
\text { sate } \\
\text { ( } \mu \mathrm{g} / \mathrm{L})\end{array}$ & $\begin{array}{c}\text { Amino- } \\
\text { meth- } \\
\text { ylphos- } \\
\text { phonic } \\
\text { acid } \\
\text { ( } \mu \mathrm{g} / \mathrm{L})\end{array}$ & $\begin{array}{c}\text { Glufosi- } \\
\text { nate } \\
(\mu \mathrm{g} / \mathrm{L})\end{array}$ \\
\hline 393405084203701 & URB-12 at Carlisle, Ohio & $\mathrm{R}$ & $11 / 06 / 01$ & 1100 & $<0.10$ & $<0.10$ & $<0.10$ \\
\hline 393453084131801 & URB-13 at Carlisle, Ohio & $\mathrm{R}$ & $11 / 05 / 01$ & 1400 & $<.10$ & $<.10$ & $<.10$ \\
\hline 393812084240001 & REF-02 at Germantown Dam, Ohio & $\mathrm{R}$ & $11 / 07 / 01$ & 1100 & $<.10$ & $<.10$ & $<.10$ \\
\hline \multirow[t]{2}{*}{393944084120700} & Holes Creek at Huffman Park at Kettering, Ohio & $\mathrm{R}$ & $08 / 15 / 01$ & 1400 & $<.10$ & $<.10$ & $<.10$ \\
\hline & & $\mathrm{R}$ & $09 / 20 / 01$ & 1200 & $<.10$ & $<.10$ & $<.10$ \\
\hline \multirow[t]{2}{*}{393956083575601} & URB-23 at Xenia, Ohio & $\mathrm{R}$ & $10 / 25 / 01$ & 1200 & $<.10$ & $<.10$ & $<.10$ \\
\hline & & LD & $10 / 25 / 01$ & 1200 & $<.10$ & $<.10$ & $<.10$ \\
\hline 394025084132501 & URB-14 at West Carrollton, Ohio & $\mathrm{R}$ & $10 / 31 / 01$ & 1200 & $<.10$ & $<.10$ & $<.10$ \\
\hline \multirow[t]{2}{*}{394103084124901} & URB-18 at Moraine, Ohio & $\mathrm{R}$ & $10 / 31 / 01$ & 1600 & $<.10$ & $<.10$ & $<.10$ \\
\hline & & FR & $10 / 31 / 01$ & 1605 & $<.10$ & $<.10$ & $<.10$ \\
\hline 394234084024301 & URB-16 at Beavercreek, Ohio & $\mathrm{R}$ & $10 / 22 / 01$ & 1600 & $<.10$ & $<.10$ & $<.10$ \\
\hline 394256084040701 & URB-19 at Beavercreek, Ohio & $\mathrm{R}$ & $10 / 23 / 01$ & 1200 & $<.10$ & $<.10$ & $<.10$ \\
\hline 395244084011901 & & LD & $12 / 03 / 01$ & 1500 & $<.10$ & $<.10$ & $<.10$ \\
\hline 395247084015801 & URB-25 at Crystal Lakes, Ohio & $\mathrm{R}$ & $12 / 03 / 01$ & 1100 & $<.10$ & $<.10$ & $<.10$ \\
\hline 395614084020301 & URB-24 at New Carlisle, Ohio & $\mathrm{R}$ & $10 / 17 / 01$ & 1400 & $<.10$ & $<.10$ & $<.10$ \\
\hline 400642083441601 & URB-21 at Ur, Ohio & $\mathrm{R}$ & $10 / 03 / 01$ & 1400 & $<.10$ & $<.10$ & $<.10$ \\
\hline \multicolumn{8}{|c|}{ Lake Erie-Lake St. Clair Drainage Surface Water (BET) } \\
\hline \multirow[t]{2}{*}{04161820} & Clinton River at Sterling Heights, Michigan & $\mathrm{R}$ & $08 / 29 / 01$ & 1000 & .15 & .28 & $<.10$ \\
\hline & & $\mathrm{R}$ & $09 / 18 / 01$ & 1030 & $<.10$ & $<.10$ & $<.10$ \\
\hline \multirow[t]{2}{*}{04175600} & River Raisin near Manchester, Michigan & $\mathrm{R}$ & $08 / 29 / 01$ & 1330 & $<.10$ & $<.10$ & $<.10$ \\
\hline & & $\mathrm{R}$ & $09 / 18 / 01$ & 1400 & $<.10$ & $<.10$ & $<.10$ \\
\hline 04178000 & St. Joseph River near Newville, Indiana & $\mathrm{R}$ & 08/28/01 & 1400 & $<.10$ & $<.10$ & $<.10$ \\
\hline
\end{tabular}


Table 5. Analytical results for glyphosate, aminomethylphosphonic acid, and glufosinate in National Water-Quality Assessment (NAWQA) Program surface- and ground-water studies, 2001-06.-Continued

[Location of NAWQA study units shown in figure 4. Sample type: FB, field blank; FR, field replicate; R, regular; LD, laboratory duplicate; --, no data; <, less than; $\mu \mathrm{g} / \mathrm{L}$, micrograms per liter]

\begin{tabular}{|c|c|c|c|c|c|c|c|}
\hline \multirow[b]{2}{*}{$\begin{array}{c}\text { Site } \\
\text { number }\end{array}$} & \multirow[b]{2}{*}{ Site location } & \multirow[b]{2}{*}{$\begin{array}{c}\text { Sample } \\
\text { type }\end{array}$} & \multirow[b]{2}{*}{$\begin{array}{c}\text { Date of } \\
\text { collection } \\
\text { (day/ } \\
\text { month/ } \\
\text { year) }\end{array}$} & \multirow[b]{2}{*}{$\begin{array}{c}\text { Col- } \\
\text { lection } \\
\text { time } \\
\text { (24- } \\
\text { hour) }\end{array}$} & \multicolumn{3}{|c|}{ Concentrations } \\
\hline & & & & & $\begin{array}{c}\text { Glypho- } \\
\text { sate } \\
\text { (pg/L) }\end{array}$ & $\begin{array}{c}\text { Amino- } \\
\text { meth- } \\
\text { ylphos- } \\
\text { phonic } \\
\text { acid } \\
(\mu \mathrm{g} / \mathrm{L})\end{array}$ & $\begin{array}{c}\text { Glufosi- } \\
\text { nate } \\
\text { ( } \mu \mathrm{g} / \mathrm{L})\end{array}$ \\
\hline \multirow[t]{2}{*}{04186500} & Auglaize River near Ft. Jennings, Ohio & $\mathrm{R}$ & 08/28/01 & 1130 & $<.10$ & .57 & $<.10$ \\
\hline & & $\mathrm{R}$ & 09/17/01 & 1300 & $<.10$ & $<.10$ & $<.10$ \\
\hline \multirow[t]{2}{*}{04193500} & Maumee River at Waterville, Ohio & $\mathrm{R}$ & 08/30/01 & 0900 & .12 & .46 & $<.10$ \\
\hline & & $\mathrm{LD}$ & 08/30/01 & 0900 & $<.10$ & .66 & $<.10$ \\
\hline \multirow[t]{16}{*}{094196783} & Las Vegas Wash below Flamingo Wash & $\mathrm{R}$ & $10 / 09 / 02$ & 0930 & .75 & 62 & $<.10$ \\
\hline & confluence near Las Vegas, Nevada & $\mathrm{R}$ & $10 / 24 / 02$ & 0930 & .68 & .23 & $<.10$ \\
\hline & & $\mathrm{R}$ & $11 / 14 / 02$ & 0930 & .48 & .37 & $<.10$ \\
\hline & & $\mathrm{R}$ & $11 / 26 / 02$ & 0830 & .52 & .25 & $<.10$ \\
\hline & & LD & $11 / 26 / 02$ & 0830 & .55 & .23 & $<.10$ \\
\hline & & $\mathrm{R}$ & $12 / 16 / 02$ & 1030 & $<.10$ & .30 & $<.10$ \\
\hline & & $\mathrm{R}$ & $12 / 30 / 02$ & 1000 & $<.10$ & .18 & $<.10$ \\
\hline & & FR & $01 / 28 / 03$ & 0950 & $<.10$ & $<.10$ & $<.10$ \\
\hline & & LD & 01/28/03 & 0950 & $<.10$ & $<.10$ & $<.10$ \\
\hline & & $\mathrm{R}$ & 02/10/03 & 1000 & $<.10$ & .14 & $<.10$ \\
\hline & & LD & 02/10/03 & 1000 & $<.10$ & .15 & $<.10$ \\
\hline & & $\mathrm{R}$ & $02 / 24 / 03$ & 0830 & $<.10$ & .33 & $<.10$ \\
\hline & & LD & $02 / 24 / 03$ & 0830 & $<.10$ & .25 & $<.10$ \\
\hline & & FB & 03/12/03 & 0845 & $<.10$ & $<.10$ & $<.10$ \\
\hline & & $\mathrm{R}$ & $03 / 12 / 03$ & 0850 & .34 & .40 & $<.10$ \\
\hline & & LD & $03 / 12 / 03$ & 0850 & .34 & .42 & $<.10$ \\
\hline
\end{tabular}


Table 5. Analytical results for glyphosate, aminomethylphosphonic acid, and glufosinate in National Water-Quality Assessment (NAWQA) Program surface- and ground-water studies, 2001-06.-Continued

[Location of NAWQA study units shown in figure 4. Sample type: FB, field blank; FR, field replicate; R, regular; LD, laboratory duplicate; --, no data; <, less than; $\mu \mathrm{g} / \mathrm{L}$, micrograms per liter]

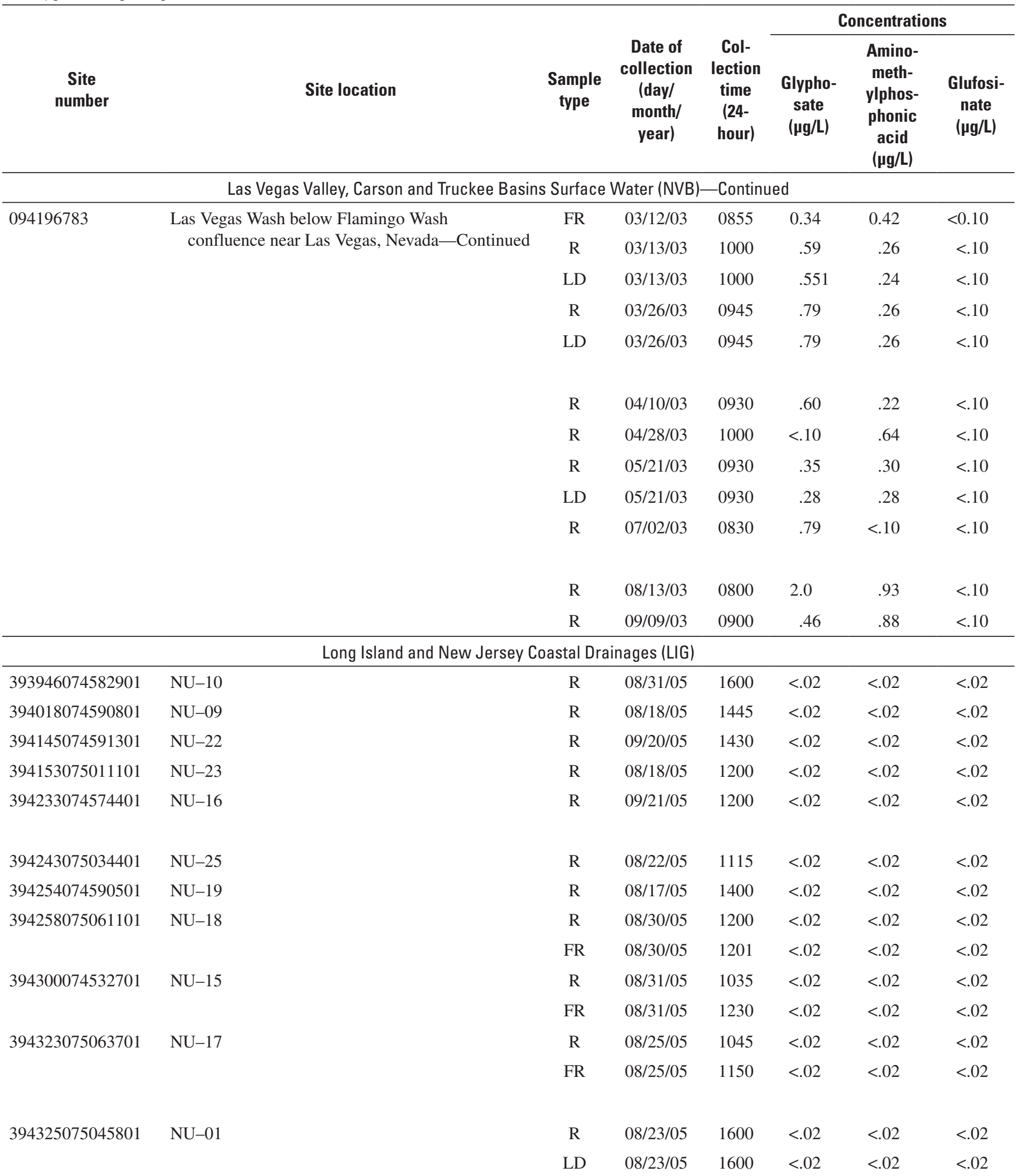


Table 5. Analytical results for glyphosate, aminomethylphosphonic acid, and glufosinate in National Water-Quality Assessment (NAWQA) Program surface- and ground-water studies, 2001-06.-Continued

[Location of NAWQA study units shown in figure 4. Sample type: FB, field blank; FR, field replicate; R, regular; LD, laboratory duplicate; --, no data; <, less than; $\mu \mathrm{g} / \mathrm{L}$, micrograms per liter]

\begin{tabular}{|c|c|c|c|c|c|c|c|}
\hline \multirow[b]{2}{*}{$\begin{array}{c}\text { Site } \\
\text { number }\end{array}$} & \multirow[b]{2}{*}{ Site location } & \multirow[b]{2}{*}{$\begin{array}{l}\text { Sample } \\
\text { type }\end{array}$} & \multirow[b]{2}{*}{$\begin{array}{l}\text { Date of } \\
\text { collection } \\
\text { (day/ } \\
\text { month/ } \\
\text { year) }\end{array}$} & \multirow[b]{2}{*}{$\begin{array}{c}\text { Col- } \\
\text { lection } \\
\text { time } \\
\text { (24- } \\
\text { hour) }\end{array}$} & \multicolumn{3}{|c|}{ Concentrations } \\
\hline & & & & & $\begin{array}{c}\text { Glypho- } \\
\text { sate } \\
(\mu \mathrm{g} / \mathrm{L})\end{array}$ & $\begin{array}{l}\text { Amino- } \\
\text { meth- } \\
\text { ylphos- } \\
\text { phonic } \\
\text { acid } \\
\text { ( } \mathrm{gg} / \mathrm{L})\end{array}$ & $\begin{array}{c}\text { Glufosi- } \\
\text { nate } \\
\text { ( } \mu \mathrm{g} / \mathrm{L})\end{array}$ \\
\hline \multicolumn{8}{|c|}{ Long Island and New Jersey Coastal Drainages (LIG)_Continued } \\
\hline 394340075012701 & NU-08 & $\mathrm{R}$ & 08/17/05 & 1100 & $<0.02$ & $<0.02$ & $<0.02$ \\
\hline 394342075040301 & NU-02 & $\mathrm{R}$ & 08/23/05 & 1300 & $<.02$ & $<.02$ & $<.02$ \\
\hline 394348074595301 & NU-13 & $\mathrm{R}$ & 08/16/05 & 1530 & $<.02$ & $<.02$ & $<.02$ \\
\hline 394428075044601 & NU-03 & $\mathrm{R}$ & $08 / 23 / 05$ & 1050 & $<.02$ & $<.02$ & $<.02$ \\
\hline 394446075031001 & NU-29 & $\mathrm{R}$ & 08/22/05 & 1400 & $<.02$ & $<.02$ & $<.02$ \\
\hline 394457075040001 & & FR & 08/24/05 & 1120 & $<.02$ & $<.02$ & $<.02$ \\
\hline 394504075051001 & NU-05 & $\mathrm{R}$ & $08 / 24 / 05$ & 1350 & $<.02$ & $<.02$ & $<.02$ \\
\hline 394507075023801 & NU-30 & $\mathrm{R}$ & $08 / 25 / 05$ & 1530 & $<.02$ & $<.02$ & $<.02$ \\
\hline 394528075004301 & NU-06 & $\mathrm{R}$ & 08/16/05 & 1130 & $<.02$ & $<.02$ & $<.02$ \\
\hline 394604075003601 & NU-11 & $\mathrm{R}$ & 09/20/05 & 1120 & $<.02$ & $<.02$ & $<.02$ \\
\hline 394606074591801 & NU-07 & $\mathrm{R}$ & $08 / 15 / 05$ & 1440 & $<.02$ & .18 & $<.02$ \\
\hline 394657074554501 & NU-26 & $\mathrm{R}$ & 08/11/05 & 1500 & $<.02$ & .16 & $<.02$ \\
\hline \multirow[t]{2}{*}{400000074000094} & Equipment blank & FB & $06 / 21 / 05$ & 1120 & $<.02$ & $<.02$ & $<.02$ \\
\hline & & FB & $06 / 21 / 05$ & 1335 & $<.02$ & $<.02$ & $<.02$ \\
\hline \multicolumn{8}{|c|}{ Lower Illinois River Basin Ground Water (ILG) } \\
\hline 383214089571501 & $\begin{array}{l}\text { 1N8W14-3.f1 SIL-25 Whiteside Elementary } \\
\text { School }\end{array}$ & $\mathrm{R}$ & 08/03/05 & 1400 & $<.02$ & $<.02$ & $<.02$ \\
\hline 383321089594601 & 1N8W9-6.h1 SIL-23 Wolf Branch Middle School & $\mathrm{R}$ & 08/03/05 & 1000 & $<.02$ & $<.02$ & $<.02$ \\
\hline \multirow[t]{2}{*}{383445089535701} & 2N7W32-3.e1 SIL-27 Evans Elementary School & $\mathrm{R}$ & 08/01/05 & 1300 & $<.02$ & $<.02$ & $<.02$ \\
\hline & & FR & 08/01/05 & 1305 & $<.02$ & $<.02$ & $<.02$ \\
\hline \multirow[t]{2}{*}{383453089545001} & 2N7W31-2.g1 SIL-27A O’Fallon Public Library & $\mathrm{R}$ & 08/04/05 & 0900 & $<.02$ & $<.02$ & $<.02$ \\
\hline & & FR & 08/04/05 & 0901 & $<.02$ & $<.02$ & $<.02$ \\
\hline 383847090003501 & 2N8W5-3.b1 & $\mathrm{R}$ & 07/12/05 & 1100 & $<.02$ & $<.02$ & $<.02$ \\
\hline 384058090010601 & 3N9W29-7.f1 & $\mathrm{R}$ & $07 / 12 / 05$ & 1500 & $<.02$ & $<.02$ & $<.02$ \\
\hline
\end{tabular}


Table 5. Analytical results for glyphosate, aminomethylphosphonic acid, and glufosinate in National Water-Quality Assessment (NAWQA) Program surface- and ground-water studies, 2001-06.-Continued

[Location of NAWQA study units shown in figure 4. Sample type: FB, field blank; FR, field replicate; R, regular; LD, laboratory duplicate; --, no data; <, less than; $\mu \mathrm{g} / \mathrm{L}$, micrograms per liter]

\begin{tabular}{|c|c|c|c|c|c|c|c|}
\hline \multirow[b]{2}{*}{$\begin{array}{c}\text { Site } \\
\text { number }\end{array}$} & \multirow[b]{2}{*}{ Site location } & \multirow[b]{2}{*}{$\begin{array}{c}\text { Sample } \\
\text { type }\end{array}$} & \multirow[b]{2}{*}{$\begin{array}{c}\text { Date of } \\
\text { collection } \\
\text { (day/ } \\
\text { month/ } \\
\text { year) }\end{array}$} & \multirow[b]{2}{*}{$\begin{array}{c}\text { Col- } \\
\text { lection } \\
\text { time } \\
\text { (24- } \\
\text { hour) }\end{array}$} & \multicolumn{3}{|c|}{ Concentrations } \\
\hline & & & & & $\begin{array}{c}\text { Glypho- } \\
\text { sate } \\
\text { ( } \mu \mathrm{g} / \mathrm{L})\end{array}$ & $\begin{array}{c}\text { Amino- } \\
\text { meth- } \\
\text { ylphos- } \\
\text { phonic } \\
\text { acid } \\
(\mu \mathrm{g} / \mathrm{L})\end{array}$ & $\begin{array}{c}\text { Glufosi- } \\
\text { nate } \\
\text { ( } \mu \mathrm{g} / \mathrm{L})\end{array}$ \\
\hline \multicolumn{8}{|c|}{ Lower Illinois River Basin Ground Water (ILG)_Continued } \\
\hline 384323090405801 & 46N3E22-8.b2 SMO-12 Ozark Way & $\mathrm{R}$ & $08 / 09 / 05$ & 0900 & $<0.02$ & $<0.02$ & $<0.02$ \\
\hline 384411090354601 & 46N4E17-3.a1 SMO-14 / Ramona Lane & $\mathrm{R}$ & $07 / 27 / 05$ & 1000 & $<.02$ & $<.02$ & $<.02$ \\
\hline \multirow[t]{2}{*}{384422090420701} & 46N3E17-1.b1 SMO-02 Sports Park & $\mathrm{R}$ & $08 / 09 / 05$ & 1400 & $<.02$ & $<.02$ & $<.02$ \\
\hline & & LD & $08 / 09 / 05$ & 1400 & .06 & $<.02$ & $<.02$ \\
\hline 384504090394701 & 46N3E10-1.a1 SMO-10 / Strehl Fields & $\mathrm{R}$ & $07 / 27 / 05$ & 1500 & $<.02$ & $<.02$ & $<.02$ \\
\hline 384515089591801 & 4N8W33-2.f1 SIL-28 / Olivia Lane & $\mathrm{R}$ & 08/08/05 & 1500 & $<.02$ & $<.02$ & $<.02$ \\
\hline 384546089575801 & 4N8W27-1.b1 SIL-21 Public Works Garage & $\mathrm{R}$ & $08 / 02 / 05$ & 0900 & $<.02$ & .16 & $<.02$ \\
\hline \multirow[t]{2}{*}{384613089572001} & 4N8W26-4.f1 SIL-30 South Station Road & $\mathrm{R}$ & $08 / 02 / 05$ & 1300 & $<.02$ & $<.02$ & $<.02$ \\
\hline & & $\mathrm{LD}$ & 08/02/05 & 1300 & $<.02$ & $<.02$ & $<.02$ \\
\hline \multirow[t]{2}{*}{384619090382701} & 46N3E2-7.d1 SMO-08 Woodlands Sports Park & $\mathrm{R}$ & $07 / 26 / 05$ & 0900 & $<.02$ & $<.02$ & $<.02$ \\
\hline & & FR & $07 / 26 / 05$ & 0901 & $<.02$ & $<.02$ & $<.02$ \\
\hline 384629090350901 & 46N4E4-8.f1 XMO-17A Covenent Park & $\mathrm{R}$ & 08/10/05 & 1500 & $<.02$ & $<.02$ & $<.02$ \\
\hline 384634090430201 & 46N3E5-8.f1 SMO-05 Stoney Brook Drive & $\mathrm{R}$ & $07 / 25 / 05$ & 1400 & $<.02$ & $<.02$ & $<.02$ \\
\hline 384635090355101 & 46N4E5-5.g1 SMO-18 Oak Creek Park & $\mathrm{R}$ & 08/10/05 & 0900 & $<.02$ & .35 & $<.02$ \\
\hline \multirow[t]{2}{*}{384646090375501} & 47N3E36-3.a1 SMO-09A Rabbit Run Park & $\mathrm{R}$ & $07 / 26 / 05$ & 1400 & $<.02$ & $<.02$ & $<.02$ \\
\hline & & FR & $07 / 26 / 05$ & 1405 & $<.02$ & $<.02$ & $<.02$ \\
\hline 384656089582001 & 4N8W22-3.e1 & $\mathrm{R}$ & $07 / 13 / 05$ & 1000 & $<.02$ & $<.02$ & $<.02$ \\
\hline 384659090003701 & 4N8W20-4.e1 & $\mathrm{R}$ & $07 / 14 / 05$ & 0900 & $<.02$ & $<.02$ & $<.02$ \\
\hline 384714089585301 & 4N8W22-7.g1 & $\mathrm{R}$ & $07 / 13 / 05$ & 1500 & $<.02$ & $<.02$ & $<.02$ \\
\hline 384734090384801 & 47N3E35.2.h1 & $\mathrm{R}$ & $07 / 28 / 05$ & 0900 & $<.02$ & $<.02$ & $<.02$ \\
\hline 384955089473001 & 5N6W32-4.a1 MRN-1 & $\mathrm{R}$ & 09/07/05 & 1100 & $<.02$ & $<.02$ & $<.02$ \\
\hline 385446090015901 & 5N8W6-5.b1 & $\mathrm{R}$ & $07 / 11 / 05$ & 1400 & $<.02$ & $<.02$ & $<.02$ \\
\hline \multirow[t]{2}{*}{385735089513901} & 6N7W22-5.g1 WDN-1 & $\mathrm{R}$ & 09/07/05 & 1500 & $<.02$ & $<.02$ & $<.02$ \\
\hline & & $\mathrm{R}$ & $09 / 07 / 05$ & 1500 & $<.02$ & $<.02$ & $<.02$ \\
\hline \multicolumn{8}{|c|}{ Mississippi Embayment Surface Water (RCM) } \\
\hline \multirow[t]{3}{*}{07288650} & Bogue Phalia near Leland, Mississippi & $\mathrm{R}$ & $10 / 13 / 04$ & 1115 & .59 & 1.1 & $<.02$ \\
\hline & & $\mathrm{R}$ & $11 / 03 / 04$ & 1130 & .35 & 1.8 & $<.02$ \\
\hline & & $\mathrm{R}$ & $12 / 07 / 04$ & 1035 & .28 & 1.1 & $<.02$ \\
\hline
\end{tabular}


Table 5. Analytical results for glyphosate, aminomethylphosphonic acid, and glufosinate in National Water-Quality Assessment (NAWQA) Program surface- and ground-water studies, 2001-06.-Continued

[Location of NAWQA study units shown in figure 4. Sample type: FB, field blank; FR, field replicate; R, regular; LD, laboratory duplicate; --, no data; <, less than; $\mu \mathrm{g} / \mathrm{L}$, micrograms per liter]

\begin{tabular}{|c|c|c|c|c|c|c|c|}
\hline \multirow[b]{2}{*}{$\begin{array}{c}\text { Site } \\
\text { number }\end{array}$} & \multirow[b]{2}{*}{ Site location } & \multirow[b]{2}{*}{$\begin{array}{c}\text { Sample } \\
\text { type }\end{array}$} & \multirow[b]{2}{*}{$\begin{array}{c}\text { Date of } \\
\text { collection } \\
\text { (day/ } \\
\text { month/ } \\
\text { year) }\end{array}$} & \multirow[b]{2}{*}{$\begin{array}{c}\text { Col- } \\
\text { lection } \\
\text { time } \\
\text { (24- } \\
\text { hour) }\end{array}$} & \multicolumn{3}{|c|}{ Concentrations } \\
\hline & & & & & $\begin{array}{c}\text { Glypho- } \\
\text { sate } \\
\text { (pg/L) }\end{array}$ & $\begin{array}{c}\text { Amino- } \\
\text { meth- } \\
\text { ylphos- } \\
\text { phonic } \\
\text { acid } \\
(\mu \mathrm{g} / \mathrm{L})\end{array}$ & $\begin{array}{c}\text { Glufosi- } \\
\text { nate } \\
\text { ( } \mathrm{\mu g} / \mathrm{L})\end{array}$ \\
\hline \multirow{18}{*}{07288650} & Continued & $\mathrm{R}$ & $01 / 04 / 05$ & 1015 & .12 & 1.5 & $<.02$ \\
\hline & & FB & 02/08/05 & 0830 & $<.02$ & $<.02$ & $<.02$ \\
\hline & & $\mathrm{LD}$ & 02/08/05 & 0830 & $<.02$ & $<.02$ & $<.02$ \\
\hline & & $\mathrm{R}$ & 03/07/05 & 1110 & .38 & 1.7 & $<.02$ \\
\hline & & $\mathrm{R}$ & $03 / 21 / 05$ & 0910 & .11 & $<.02$ & $<.02$ \\
\hline & & $\mathrm{R}$ & $03 / 22 / 05$ & 1110 & 2.4 & 1.5 & $<.02$ \\
\hline & & $\mathrm{R}$ & 03/28/05 & 1135 & 1.0 & 2.6 & $<.02$ \\
\hline & & $\mathrm{R}$ & $04 / 04 / 05$ & 1139 & .17 & 2.4 & $<.02$ \\
\hline & & LD & $04 / 04 / 05$ & 1139 & .17 & 1.9 & $<.02$ \\
\hline & & FR & $04 / 04 / 05$ & 1140 & .22 & 2.1 & $<.02$ \\
\hline & & FB & 06/07/05 & 1100 & $<.02$ & $<.02$ & $<.02$ \\
\hline & & $\mathrm{R}$ & 06/07/05 & 1100 & 1.1 & 3.9 & $<.02$ \\
\hline & & FB & 06/20/05 & 1100 & $<.02$ & $<.02$ & $<.02$ \\
\hline & & $\mathrm{R}$ & 06/20/05 & 1100 & 1.8 & 8.7 & $<.02$ \\
\hline & & $\mathrm{R}$ & 07/05/05 & 1000 & 1.7 & 5.0 & $<.02$ \\
\hline & & $\mathrm{R}$ & 07/20/05 & 1200 & 1.1 & 3.9 & $<.02$ \\
\hline & & LD & 07/20/05 & 1200 & 1.5 & 4.6 & $<.02$ \\
\hline & & $\mathrm{R}$ & 08/08/05 & 1200 & 1.8 & 4.9 & $<.02$ \\
\hline & & $\mathrm{R}$ & 08/09/05 & 0900 & 2.3 & 4.1 & $<.02$ \\
\hline
\end{tabular}


Table 5. Analytical results for glyphosate, aminomethylphosphonic acid, and glufosinate in National Water-Quality Assessment (NAWQA) Program surface- and ground-water studies, 2001-06.-Continued

[Location of NAWQA study units shown in figure 4. Sample type: FB, field blank; FR, field replicate; R, regular; LD, laboratory duplicate; --, no data; <, less than; $\mu \mathrm{g} / \mathrm{L}$, micrograms per liter]

\begin{tabular}{|c|c|c|c|c|c|c|c|}
\hline \multirow[b]{2}{*}{$\begin{array}{c}\text { Site } \\
\text { number }\end{array}$} & \multirow[b]{2}{*}{ Site location } & \multirow[b]{2}{*}{$\begin{array}{l}\text { Sample } \\
\text { type }\end{array}$} & \multirow[b]{2}{*}{$\begin{array}{c}\text { Date of } \\
\text { collection } \\
\text { (day/ } \\
\text { month/ } \\
\text { year) }\end{array}$} & \multirow[b]{2}{*}{$\begin{array}{c}\text { Col- } \\
\text { lection } \\
\text { time } \\
(24- \\
\text { hour })\end{array}$} & \multicolumn{3}{|c|}{ Concentrations } \\
\hline & & & & & $\begin{array}{c}\text { Glypho- } \\
\text { sate } \\
(\mu \mathrm{g} / \mathrm{L})\end{array}$ & $\begin{array}{c}\text { Amino- } \\
\text { meth- } \\
\text { ylphos- } \\
\text { phonic } \\
\text { acid } \\
\text { ( } \mu \mathrm{g} / \mathrm{L})\end{array}$ & $\begin{array}{c}\text { Glufosi- } \\
\text { nate } \\
(\mu \mathrm{g} / \mathrm{L})\end{array}$ \\
\hline \multicolumn{8}{|c|}{ Mississippi Embayment Surface Water (RCM)—Continued } \\
\hline \multirow[t]{3}{*}{07288650} & \multirow{3}{*}{$\begin{array}{l}\text { Bogue Phalia near Leland, Mississippi- } \\
\text { Continued }\end{array}$} & $\mathrm{R}$ & 08/23/05 & 1000 & 1.4 & 6.7 & $<0.02$ \\
\hline & & $\mathrm{R}$ & $08 / 24 / 05$ & 1100 & .84 & 4.1 & $<.02$ \\
\hline & & $\mathrm{R}$ & $09 / 06 / 05$ & 1000 & .53 & 1.7 & $<.02$ \\
\hline \multirow[t]{20}{*}{07288955} & \multirow[t]{20}{*}{ Yazoo River below Steele Bayou near Long Lake } & $\mathrm{R}$ & $10 / 14 / 04$ & 1200 & .13 & .79 & $<.02$ \\
\hline & & $\mathrm{R}$ & $11 / 04 / 04$ & 1300 & .13 & .48 & $<.02$ \\
\hline & & $\mathrm{R}$ & $12 / 08 / 04$ & 1310 & .12 & .41 & $<.02$ \\
\hline & & $\mathrm{R}$ & $02 / 10 / 05$ & 1400 & .13 & .61 & $<.02$ \\
\hline & & $\mathrm{R}$ & $02 / 24 / 05$ & 1100 & $<.02$ & .23 & $<.02$ \\
\hline & & $\mathrm{R}$ & $03 / 14 / 05$ & 1200 & .17 & .43 & $<.02$ \\
\hline & & $\mathrm{R}$ & 03/23/05 & 1145 & .12 & .26 & $<.02$ \\
\hline & & $\mathrm{R}$ & 04/07/05 & 1145 & $<.02$ & .45 & $<.02$ \\
\hline & & FR & 04/07/05 & 1146 & $<.02$ & .51 & $<.02$ \\
\hline & & $\mathrm{R}$ & $04 / 21 / 05$ & 1200 & .38 & .29 & $<.02$ \\
\hline & & $\mathrm{R}$ & $05 / 24 / 05$ & 1205 & .24 & .77 & $<.02$ \\
\hline & & $\mathrm{R}$ & 06/09/05 & 1200 & $<.02$ & .61 & $<.02$ \\
\hline & & $\mathrm{R}$ & $06 / 22 / 05$ & 1000 & .08 & .52 & $<.02$ \\
\hline & & $\mathrm{R}$ & 07/07/05 & 1100 & $<.02$ & .73 & $<.02$ \\
\hline & & $\mathrm{R}$ & 07/25/05 & 1200 & .13 & 1.1 & $<.02$ \\
\hline & & $\mathrm{R}$ & 08/10/05 & 1100 & .19 & .8 & $<.02$ \\
\hline & & $\mathrm{R}$ & 09/07/05 & 1130 & .13 & .95 & $<.02$ \\
\hline & & FB & 05/04/05 & 1000 & $<.02$ & $<.02$ & $<.02$ \\
\hline & & $\mathrm{R}$ & 05/04/05 & 1020 & .09 & .44 & $<.02$ \\
\hline & & LD & 05/04/05 & 1020 & .18 & .39 & $<.02$ \\
\hline \multirow[t]{2}{*}{332440090502101} & \multirow[t]{2}{*}{ FO135 Bogue Phalia—right bank } & FB & 09/15/05 & 1030 & $<.02$ & $<.02$ & $<.02$ \\
\hline & & LD & $09 / 15 / 05$ & 1030 & $<.02$ & $<.02$ & $<.02$ \\
\hline 332440090502103 & FO137 Bogue Phalia—left bank & FB & 09/15/05 & 1045 & $<.02$ & $<.02$ & $<.02$ \\
\hline 332541090503301 & FO005 Washington & $\mathrm{R}$ & $09 / 21 / 05$ & 1400 & $<.02$ & $<.02$ & $<.02$ \\
\hline
\end{tabular}


Table 5. Analytical results for glyphosate, aminomethylphosphonic acid, and glufosinate in National Water-Quality Assessment (NAWQA) Program surface- and ground-water studies, 2001-06.-Continued

[Location of NAWQA study units shown in figure 4. Sample type: FB, field blank; FR, field replicate; R, regular; LD, laboratory duplicate; --, no data; <, less than; $\mu \mathrm{g} / \mathrm{L}$, micrograms per liter]

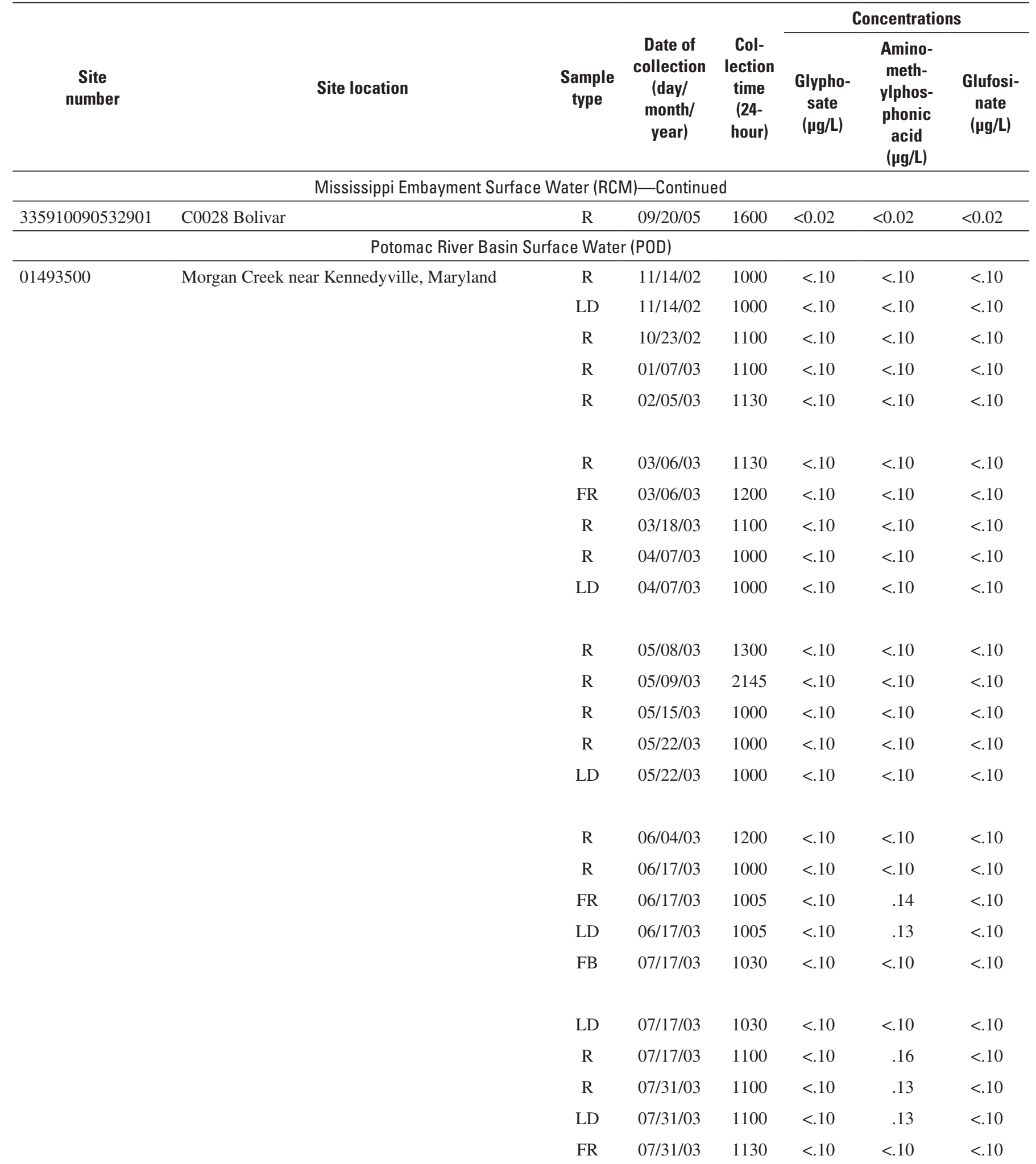


Table 5. Analytical results for glyphosate, aminomethylphosphonic acid, and glufosinate in National Water-Quality Assessment (NAWQA) Program surface- and ground-water studies, 2001-06.-Continued

[Location of NAWQA study units shown in figure 4. Sample type: FB, field blank; FR, field replicate; R, regular; LD, laboratory duplicate; --, no data; <, less than; $\mu \mathrm{g} / \mathrm{L}$, micrograms per liter]

\begin{tabular}{|c|c|c|c|c|c|c|c|}
\hline \multirow[b]{2}{*}{$\begin{array}{c}\text { Site } \\
\text { number }\end{array}$} & \multirow[b]{2}{*}{ Site location } & \multirow[b]{2}{*}{$\begin{array}{l}\text { Sample } \\
\text { type }\end{array}$} & \multirow[b]{2}{*}{$\begin{array}{c}\text { Date of } \\
\text { collection } \\
\text { (day/ } \\
\text { month/ } \\
\text { year) }\end{array}$} & \multirow[b]{2}{*}{$\begin{array}{l}\text { Col- } \\
\text { lection } \\
\text { time } \\
\text { (24- } \\
\text { hour) }\end{array}$} & \multicolumn{3}{|c|}{ Concentrations } \\
\hline & & & & & $\begin{array}{c}\text { Glypho- } \\
\text { sate } \\
(\mu \mathrm{g} / \mathrm{L})\end{array}$ & $\begin{array}{l}\text { Amino- } \\
\text { meth- } \\
\text { ylphos- } \\
\text { phonic } \\
\text { acid } \\
(\mu \mathrm{g} / \mathrm{L})\end{array}$ & $\begin{array}{c}\text { Glufosi- } \\
\text { nate } \\
(\mu \mathrm{g} / \mathrm{L})\end{array}$ \\
\hline \multicolumn{8}{|c|}{ Potomac River Basin Surface Water (POD)_Continued } \\
\hline \multirow[t]{7}{*}{01493500} & \multirow{7}{*}{$\begin{array}{l}\text { Morgan Creek near Kennedyville, Maryland- } \\
\text { Continued }\end{array}$} & $\mathrm{R}$ & $08 / 14 / 03$ & 1000 & $<0.10$ & 0.21 & $<0.10$ \\
\hline & & FB & 08/14/03 & 0930 & $<.10$ & $<.10$ & $<.10$ \\
\hline & & $\mathrm{LD}$ & $08 / 14 / 03$ & 0930 & $<.10$ & $<.10$ & $<.10$ \\
\hline & & $\mathrm{R}$ & $09 / 05 / 03$ & 1000 & $<.10$ & .32 & $<.10$ \\
\hline & & $\mathrm{R}$ & 09/30/03 & 1100 & $<.10$ & .11 & $<.10$ \\
\hline & & $\mathrm{R}$ & $12 / 13 / 02$ & 1030 & $<.10$ & $<.10$ & $<.10$ \\
\hline & & $\mathrm{R}$ & $04 / 21 / 03$ & 0930 & $<.10$ & $<.10$ & $<.10$ \\
\hline \multicolumn{8}{|c|}{ Puget Sound Basin Surface Water (PUB) } \\
\hline \multirow[t]{20}{*}{12128000} & Thornton Creek near Seattle, Washington & $\mathrm{R}$ & $10 / 13 / 04$ & 1330 & .06 & .14 & $<.02$ \\
\hline & & $\mathrm{R}$ & $10 / 13 / 04$ & 1338 & $<.02$ & $<.02$ & $<.02$ \\
\hline & & FR & $10 / 13 / 04$ & 1339 & $<.02$ & $<.02$ & $<.02$ \\
\hline & & $\mathrm{R}$ & $11 / 10 / 04$ & 1230 & .05 & .15 & $<.02$ \\
\hline & & $\mathrm{LD}$ & 11/10/04 & 1230 & .10 & .11 & $<.02$ \\
\hline & & $\mathrm{R}$ & $12 / 14 / 04$ & 1340 & .07 & .15 & $<.02$ \\
\hline & & $\mathrm{R}$ & $01 / 12 / 05$ & 1310 & $<.02$ & .17 & $<.02$ \\
\hline & & $\mathrm{R}$ & $02 / 09 / 05$ & 1220 & $<.02$ & .11 & $<.02$ \\
\hline & & $\mathrm{R}$ & 03/09/05 & 1210 & $<.02$ & $<.02$ & $<.02$ \\
\hline & & $\mathrm{R}$ & 03/22/05 & 1150 & $<.02$ & $<.02$ & $<.02$ \\
\hline & & $\mathrm{R}$ & $04 / 12 / 05$ & 1210 & $<.02$ & $<.02$ & $<.02$ \\
\hline & & $\mathrm{R}$ & $04 / 28 / 05$ & 1210 & $<.02$ & $<.02$ & $<.02$ \\
\hline & & $\mathrm{R}$ & $05 / 12 / 05$ & 1210 & .14 & .08 & $<.02$ \\
\hline & & $\mathrm{R}$ & 05/24/05 & 1240 & .08 & $<.02$ & $<.02$ \\
\hline & & FR & 05/24/05 & 1241 & .06 & .07 & $<.02$ \\
\hline & & $\mathrm{R}$ & 06/01/05 & 1200 & .53 & .12 & $<.02$ \\
\hline & & $\mathrm{R}$ & 06/08/05 & 1200 & .18 & $<.02$ & $<.02$ \\
\hline & & $\mathrm{R}$ & $06 / 15 / 05$ & 1330 & .08 & .11 & $<.02$ \\
\hline & & $\mathrm{R}$ & $06 / 21 / 05$ & 0950 & .05 & .11 & $<.02$ \\
\hline & & LD & 06/21/05 & 0950 & .05 & .10 & $<.02$ \\
\hline
\end{tabular}


Table 5. Analytical results for glyphosate, aminomethylphosphonic acid, and glufosinate in National Water-Quality Assessment (NAWQA) Program surface- and ground-water studies, 2001-06.-Continued

[Location of NAWQA study units shown in figure 4. Sample type: FB, field blank; FR, field replicate; R, regular; LD, laboratory duplicate; --, no data; <, less than; $\mu \mathrm{g} / \mathrm{L}$, micrograms per liter]

\begin{tabular}{|c|c|c|c|c|c|c|c|}
\hline \multirow[b]{2}{*}{$\begin{array}{c}\text { Site } \\
\text { number }\end{array}$} & \multirow[b]{2}{*}{ Site location } & \multirow[b]{2}{*}{$\begin{array}{l}\text { Sample } \\
\text { type }\end{array}$} & \multirow[b]{2}{*}{$\begin{array}{l}\text { Date of } \\
\text { collection } \\
\text { (day/ } \\
\text { month/ } \\
\text { year) }\end{array}$} & \multirow[b]{2}{*}{$\begin{array}{c}\text { Col- } \\
\text { lection } \\
\text { time } \\
\text { (24- } \\
\text { hour) }\end{array}$} & \multicolumn{3}{|c|}{ Concentrations } \\
\hline & & & & & $\begin{array}{c}\text { Glypho- } \\
\text { sate } \\
\text { ( } \mu \mathrm{g} / \mathrm{L})\end{array}$ & $\begin{array}{l}\text { Amino- } \\
\text { meth- } \\
\text { ylphos- } \\
\text { phonic } \\
\text { acid } \\
\text { ( } \mathrm{gg} / \mathrm{L})\end{array}$ & $\begin{array}{c}\text { Glufosi- } \\
\text { nate } \\
\text { ( } \mu \mathrm{g} / \mathrm{L})\end{array}$ \\
\hline \multirow[t]{10}{*}{12128000} & \multirow{2}{*}{$\begin{array}{l}\text { Thornton Creek near Seattle, Washington- } \\
\text { Continued }\end{array}$} & $\mathrm{R}$ & $06 / 28 / 05$ & 0930 & 0.07 & 0.09 & $<0.02$ \\
\hline & & $\mathrm{LD}$ & $06 / 28 / 05$ & 0930 & .06 & $<.02$ & $<.02$ \\
\hline & & $\mathrm{R}$ & 07/13/05 & 1000 & $<.02$ & .07 & $<.02$ \\
\hline & & FR & 07/13/05 & 1008 & $<.02$ & $<.02$ & $<.02$ \\
\hline & & $\mathrm{R}$ & $07 / 25 / 05$ & 1050 & .03 & .11 & $<.02$ \\
\hline & & $\mathrm{R}$ & 08/10/05 & 1140 & $<.02$ & .20 & $<.02$ \\
\hline & & $\mathrm{R}$ & 08/16/05 & 1140 & $<.02$ & .09 & $<.02$ \\
\hline & & $\mathrm{R}$ & $08 / 23 / 05$ & 1130 & $<.02$ & $<.02$ & $<.02$ \\
\hline & & $\mathrm{R}$ & 09/06/05 & 1230 & .45 & .33 & $<.02$ \\
\hline & & $\mathrm{R}$ & 09/20/05 & 1200 & .09 & .18 & $<.02$ \\
\hline \multicolumn{8}{|c|}{ Puget Sound Basin Ground Water (PUG) } \\
\hline 470040122463601 & $\mathrm{UR}-1$ & $\mathrm{R}$ & 08/04/05 & 1158 & $<.02$ & $<.02$ & $<.02$ \\
\hline 470040122463601 & UR-1 & $\mathrm{R}$ & 08/12/05 & 1200 & $<.02$ & $<.02$ & $<.02$ \\
\hline 470040122463601 & UR-1 & FR & 08/12/05 & 1208 & $<.02$ & $<.02$ & $<.02$ \\
\hline 470045122463801 & -- & $\mathrm{R}$ & 07/07/05 & 1310 & $<.02$ & $<.02$ & $<.02$ \\
\hline 470046122522301 & $\mathrm{UR}-08$ & $\mathrm{R}$ & 08/09/05 & 1530 & $<.02$ & $<.02$ & $<.02$ \\
\hline 470110122484201 & -- & $\mathrm{R}$ & 07/07/05 & 1040 & $<.02$ & $<.02$ & $<.02$ \\
\hline 470110122484201 & -- & LD & 07/07/05 & 1040 & $<.02$ & $<.02$ & $<.02$ \\
\hline 470112122493501 & UR-07 & $\mathrm{R}$ & 08/09/05 & 1200 & $<.02$ & $<.02$ & $<.02$ \\
\hline 470112122493501 & $\mathrm{UR}-07$ & FR & 08/09/05 & 1208 & $<.02$ & $<.02$ & $<.02$ \\
\hline 470135122202501 & UR-21 & $\mathrm{R}$ & 08/03/05 & 1420 & $<.02$ & $<.02$ & $<.02$ \\
\hline
\end{tabular}


Table 5. Analytical results for glyphosate, aminomethylphosphonic acid, and glufosinate in National Water-Quality Assessment (NAWQA) Program surface- and ground-water studies, 2001-06.-Continued

[Location of NAWQA study units shown in figure 4. Sample type: FB, field blank; FR, field replicate; R, regular; LD, laboratory duplicate; --, no data; <, less than; $\mu \mathrm{g} / \mathrm{L}$, micrograms per liter]

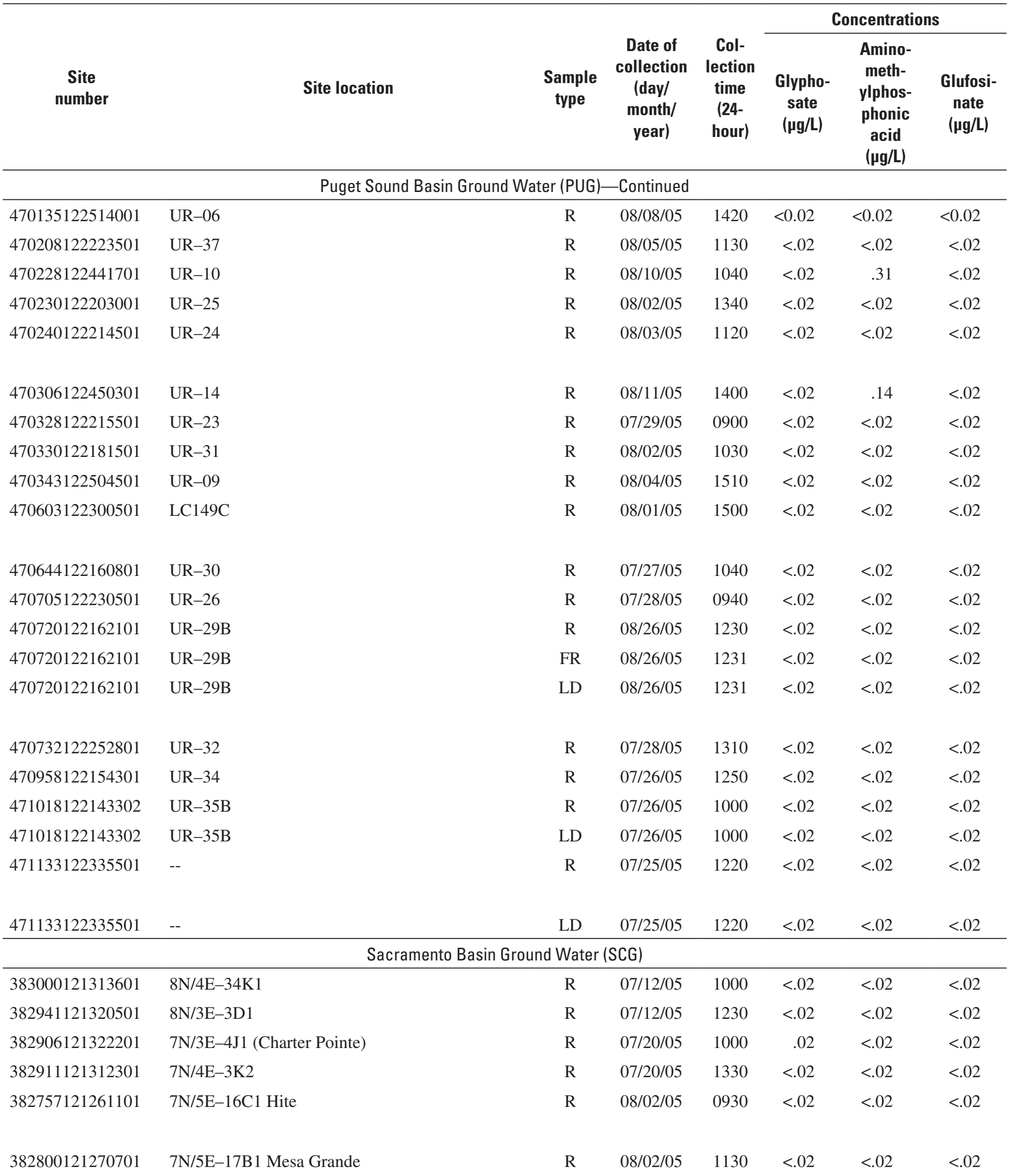


Table 5. Analytical results for glyphosate, aminomethylphosphonic acid, and glufosinate in National Water-Quality Assessment (NAWQA) Program surface- and ground-water studies, 2001-06.-Continued

[Location of NAWQA study units shown in figure 4. Sample type: FB, field blank; FR, field replicate; R, regular; LD, laboratory duplicate; --, no data; <, less than; $\mu \mathrm{g} / \mathrm{L}$, micrograms per liter]

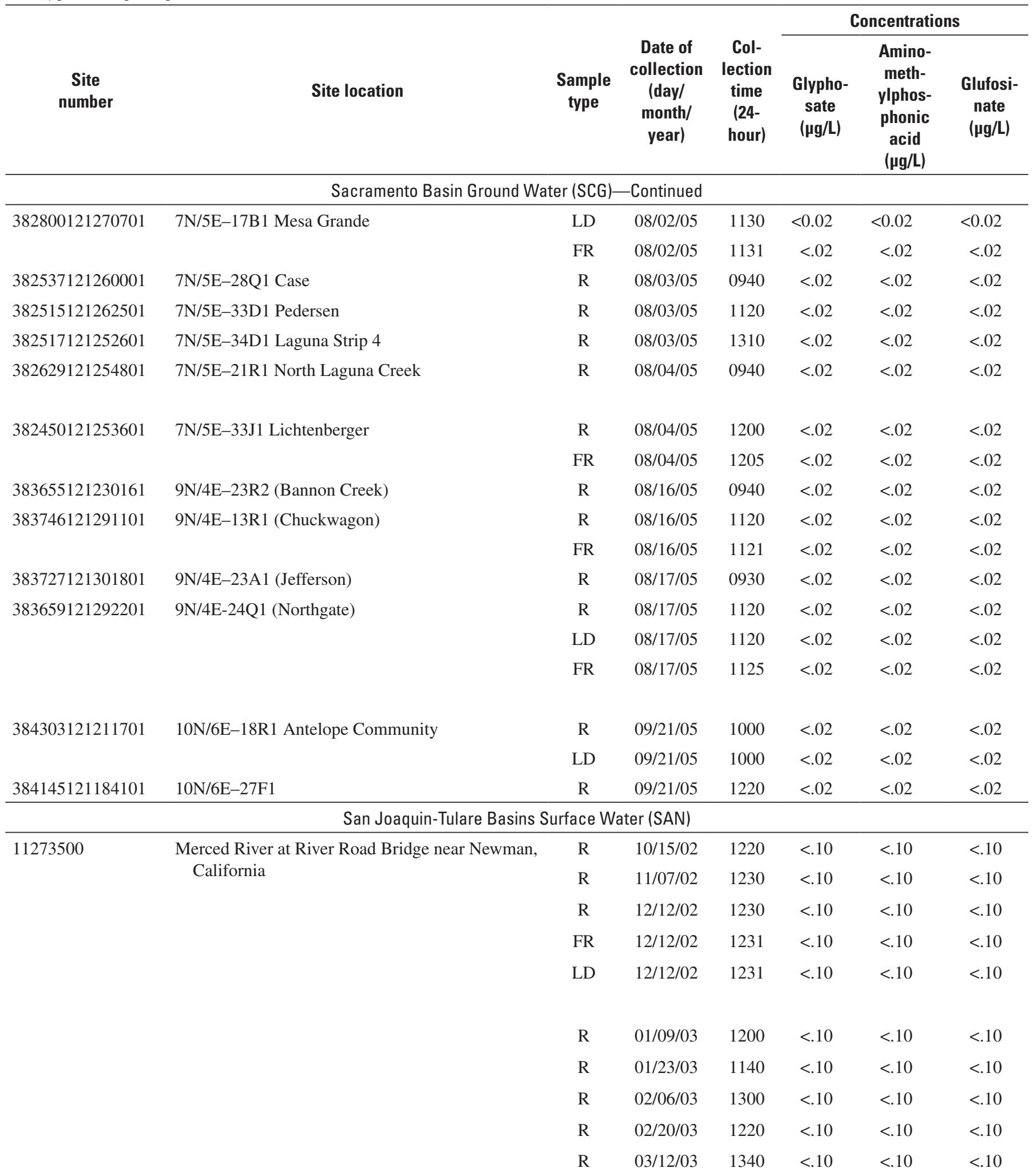


Table 5. Analytical results for glyphosate, aminomethylphosphonic acid, and glufosinate in National Water-Quality Assessment (NAWQA) Program surface- and ground-water studies, 2001-06.-Continued

[Location of NAWQA study units shown in figure 4. Sample type: FB, field blank; FR, field replicate; R, regular; LD, laboratory duplicate; --, no data; <, less than; $\mu \mathrm{g} / \mathrm{L}$, micrograms per liter]

\begin{tabular}{|c|c|c|c|c|c|c|c|}
\hline \multirow[b]{2}{*}{$\begin{array}{c}\text { Site } \\
\text { number }\end{array}$} & \multirow[b]{2}{*}{ Site location } & \multirow[b]{2}{*}{$\begin{array}{l}\text { Sample } \\
\text { type }\end{array}$} & \multirow[b]{2}{*}{$\begin{array}{c}\text { Date of } \\
\text { collection } \\
\text { (day/ } \\
\text { month/ } \\
\text { year) }\end{array}$} & \multirow[b]{2}{*}{$\begin{array}{c}\text { Col- } \\
\text { lection } \\
\text { time } \\
\text { (24- } \\
\text { hour) }\end{array}$} & \multicolumn{3}{|c|}{ Concentrations } \\
\hline & & & & & $\begin{array}{c}\text { Glypho- } \\
\text { sate } \\
\text { ( } \mu \mathrm{g} / \mathrm{L})\end{array}$ & $\begin{array}{c}\text { Amino- } \\
\text { meth- } \\
\text { ylphos- } \\
\text { phonic } \\
\text { acid } \\
\text { ( } \mu \mathrm{g} / \mathrm{L})\end{array}$ & $\begin{array}{c}\text { Glufosi- } \\
\text { nate } \\
\text { ( } \mathrm{\mu g} / \mathrm{L})\end{array}$ \\
\hline \multirow[t]{11}{*}{11273500} & \multirow{11}{*}{$\begin{array}{l}\text { Merced River at River Road Bridge near Newman, } \\
\text { California-Continued }\end{array}$} & FR & 03/12/03 & 1348 & $<0.10$ & $<0.10$ & $<0.10$ \\
\hline & & $\mathrm{R}$ & 03/28/03 & 1320 & $<.10$ & $<.10$ & $<.10$ \\
\hline & & $\mathrm{R}$ & 04/09/03 & 1330 & $<.10$ & $<.10$ & $<.10$ \\
\hline & & $\mathrm{R}$ & $04 / 29 / 03$ & 1450 & $<.10$ & $<.10$ & $<.10$ \\
\hline & & $\mathrm{R}$ & 06/11/03 & 1340 & $<.10$ & $<.10$ & $<.10$ \\
\hline & & $\mathrm{R}$ & 06/25/03 & 1350 & $<.10$ & $<.10$ & $<.10$ \\
\hline & & LD & 06/25/03 & 1350 & $<.10$ & $<.10$ & $<.10$ \\
\hline & & FR & $06 / 25 / 03$ & 1351 & $<.10$ & $<.10$ & $<.10$ \\
\hline & & $\mathrm{R}$ & 07/10/03 & 1340 & $<.10$ & $<.10$ & $<.10$ \\
\hline & & $\mathrm{R}$ & 07/24/03 & 1250 & $<.10$ & $<.10$ & $<.10$ \\
\hline & & $\mathrm{R}$ & 08/07/03 & 1340 & .18 & .22 & $<.10$ \\
\hline \multirow{10}{*}{11274538} & \multirow{10}{*}{$\begin{array}{l}\text { Orestimba Creek at River Road near Crows } \\
\text { Landing, California }\end{array}$} & $\mathrm{R}$ & $02 / 20 / 03$ & 1150 & .20 & .60 & $<.10$ \\
\hline & & $\mathrm{R}$ & 03/12/03 & 1300 & 7.5 & .69 & $<.10$ \\
\hline & & LD & 03/12/03 & 1300 & 6.8 & 1.0 & $<.10$ \\
\hline & & $\mathrm{R}$ & 03/12/03 & 1301 & 7.7 & .83 & $<.10$ \\
\hline & & FR & 03/12/03 & 1308 & $<.10$ & $<.10$ & $<.10$ \\
\hline & & LD & 03/12/03 & 1308 & $<.10$ & $<.10$ & $<.10$ \\
\hline & & $\mathrm{R}$ & 03/28/03 & 1240 & 3.0 & .61 & $<.10$ \\
\hline & & LD & 03/28/03 & 1240 & 3.1 & .61 & $<.10$ \\
\hline & & $\mathrm{R}$ & 04/09/03 & 1250 & 1.3 & .65 & $<.10$ \\
\hline & & $\mathrm{R}$ & $04 / 29 / 03$ & 1420 & .63 & .59 & $<.10$ \\
\hline
\end{tabular}


Table 5. Analytical results for glyphosate, aminomethylphosphonic acid, and glufosinate in National Water-Quality Assessment (NAWQA) Program surface- and ground-water studies, 2001-06.-Continued

[Location of NAWQA study units shown in figure 4. Sample type: FB, field blank; FR, field replicate; R, regular; LD, laboratory duplicate; --, no data; <, less than; $\mu \mathrm{g} / \mathrm{L}$, micrograms per liter]

\begin{tabular}{|c|c|c|c|c|c|c|c|}
\hline \multirow[b]{2}{*}{$\begin{array}{c}\text { Site } \\
\text { number }\end{array}$} & \multirow[b]{2}{*}{ Site location } & \multirow[b]{2}{*}{$\begin{array}{c}\text { Sample } \\
\text { type }\end{array}$} & \multirow[b]{2}{*}{$\begin{array}{c}\text { Date of } \\
\text { collection } \\
\text { (day/ } \\
\text { month/ } \\
\text { year) }\end{array}$} & \multirow[b]{2}{*}{$\begin{array}{c}\text { Col- } \\
\text { lection } \\
\text { time } \\
\text { (24- } \\
\text { hour) }\end{array}$} & \multicolumn{3}{|c|}{ Concentrations } \\
\hline & & & & & $\begin{array}{c}\text { Glypho- } \\
\text { sate } \\
\text { ( } \mathrm{gg} / \mathrm{L})\end{array}$ & $\begin{array}{l}\text { Amino- } \\
\text { meth- } \\
\text { ylphos- } \\
\text { phonic } \\
\text { acid } \\
\text { ( } \mathrm{\mu g} / \mathrm{L} \text { ) }\end{array}$ & $\begin{array}{c}\text { Glufosi- } \\
\text { nate } \\
\text { ( } \mathrm{\mu g} / \mathrm{L})\end{array}$ \\
\hline \multirow[t]{9}{*}{11274538} & Orestimba Creek at River Road near Crows & $\mathrm{R}$ & $05 / 14 / 03$ & 1420 & 1.2 & 0.58 & $<0.10$ \\
\hline & Landing, California-Continued & $\mathrm{R}$ & $05 / 14 / 03$ & 1428 & $<.10$ & $<.10$ & $<.10$ \\
\hline & & $\mathrm{R}$ & $05 / 28 / 03$ & 1220 & .10 & .32 & $<.10$ \\
\hline & & $\mathrm{R}$ & $06 / 11 / 03$ & 1300 & .34 & .50 & $<.10$ \\
\hline & & $\mathrm{LD}$ & 07/10/03 & 1310 & .22 & .45 & $<.10$ \\
\hline & & $\mathrm{R}$ & $07 / 24 / 03$ & 1200 & .38 & 1.1 & $<.10$ \\
\hline & & $\mathrm{LD}$ & $07 / 24 / 03$ & 1200 & .36 & 1.0 & $<.10$ \\
\hline & & $\mathrm{R}$ & 08/07/03 & 1300 & .83 & .66 & $<.10$ \\
\hline & & $\mathrm{R}$ & 09/04/03 & 1050 & .35 & .50 & $<.10$ \\
\hline \multirow[t]{11}{*}{11303500} & San Joaquin River near Vernalis, California & $\mathrm{R}$ & $10 / 15 / 02$ & 1020 & $<.10$ & .28 & $<.10$ \\
\hline & & $\mathrm{R}$ & 02/19/03 & 1130 & $<.10$ & .27 & $<.10$ \\
\hline & & $\mathrm{R}$ & 03/11/03 & 1240 & .16 & .36 & $<.10$ \\
\hline & & $\mathrm{R}$ & $03 / 11 / 03$ & 1248 & $<.10$ & $<.10$ & $<.10$ \\
\hline & & $\mathrm{R}$ & 03/27/03 & 1300 & $<.10$ & .24 & $<.10$ \\
\hline & & $\mathrm{R}$ & 04/08/03 & 1300 & $<.10$ & .20 & $<.10$ \\
\hline & & $\mathrm{R}$ & $04 / 29 / 03$ & 1300 & $<.10$ & .12 & $<.10$ \\
\hline & & $\mathrm{R}$ & 05/13/03 & 1330 & $<.10$ & .19 & $<.10$ \\
\hline & & $\mathrm{R}$ & $05 / 28 / 03$ & 1100 & $<.10$ & .15 & $<.10$ \\
\hline & & $\mathrm{R}$ & 06/10/03 & 1400 & $<.10$ & .21 & $<.10$ \\
\hline & & $\mathrm{R}$ & $06 / 24 / 03$ & 1220 & $<.10$ & .20 & $<.10$ \\
\hline
\end{tabular}


Table 5. Analytical results for glyphosate, aminomethylphosphonic acid, and glufosinate in National Water-Quality Assessment (NAWQA) Program surface- and ground-water studies, 2001-06.-Continued

[Location of NAWQA study units shown in figure 4. Sample type: FB, field blank; FR, field replicate; R, regular; LD, laboratory duplicate; --, no data; <, less than; $\mu \mathrm{g} / \mathrm{L}$, micrograms per liter]

\begin{tabular}{|c|c|c|c|c|c|c|c|}
\hline \multirow[b]{2}{*}{$\underset{\text { Site }}{\text { number }}$} & \multirow[b]{2}{*}{ Site location } & \multirow[b]{2}{*}{$\begin{array}{c}\text { Sample } \\
\text { type }\end{array}$} & \multirow[b]{2}{*}{$\begin{array}{c}\text { Date of } \\
\text { collection } \\
\text { (day/ } \\
\text { month/ } \\
\text { year) }\end{array}$} & \multirow[b]{2}{*}{$\begin{array}{c}\text { Col- } \\
\text { lection } \\
\text { time } \\
(24- \\
\text { hour })\end{array}$} & \multicolumn{3}{|c|}{ Concentrations } \\
\hline & & & & & $\begin{array}{l}\text { Glypho- } \\
\text { sate } \\
\text { ( } \mu \mathrm{g} / \mathrm{L})\end{array}$ & $\begin{array}{c}\text { Amino- } \\
\text { meth- } \\
\text { ylphos- } \\
\text { phonic } \\
\text { acid } \\
\text { ( } \mu \mathrm{g} / \mathrm{L})\end{array}$ & $\begin{array}{l}\text { Glufosi- } \\
\text { nate } \\
(\mu \mathrm{g} / \mathrm{L})\end{array}$ \\
\hline \multicolumn{8}{|c|}{ San Joaquin-Tulare Basins Surface Water (SAN) —Continued } \\
\hline \multirow[t]{8}{*}{11303500} & \multirow{8}{*}{$\begin{array}{l}\text { San Joaquin River near Vernalis, California- } \\
\text { Continued }\end{array}$} & $\mathrm{R}$ & 07/09/03 & 1330 & 0.18 & 0.22 & $<0.10$ \\
\hline & & $\mathrm{R}$ & $07 / 23 / 03$ & 1210 & $<.10$ & .48 & $<.10$ \\
\hline & & $\mathrm{R}$ & 08/06/03 & 1400 & $<.10$ & .56 & $<.10$ \\
\hline & & $\mathrm{R}$ & 09/03/03 & 1130 & .24 & .31 & $<.10$ \\
\hline & & $\mathrm{R}$ & $11 / 08 / 02$ & 1130 & .13 & .14 & $<.10$ \\
\hline & & LD & $11 / 08 / 02$ & 1130 & .17 & .16 & $<.10$ \\
\hline & & $\mathrm{R}$ & $01 / 08 / 03$ & 1230 & $<.10$ & .15 & $<.10$ \\
\hline & & LD & $01 / 08 / 03$ & 1230 & $<.10$ & .10 & $<.10$ \\
\hline \multicolumn{8}{|c|}{ San Joaquin-Tulare Basins Ground Water (SAG) } \\
\hline 355750119185101 & $23 \mathrm{~S} / 25 \mathrm{E}-6$ & $\mathrm{R}$ & $08 / 07 / 02$ & 1010 & $<.10$ & $<.10$ & $<.10$ \\
\hline 360310119201901 & 021S024E36N002M & $\mathrm{R}$ & $07 / 24 / 02$ & 1000 & $<.10$ & $<.10$ & $<.10$ \\
\hline 360310119201901 & 021S024E36N002M & FR & $07 / 24 / 02$ & 1005 & $<.10$ & $<.10$ & $<.10$ \\
\hline 360710119145301 & $21 \mathrm{~S} / 25 \mathrm{E}-10$ & $\mathrm{R}$ & 07/30/02 & 1350 & $<.10$ & $<.10$ & $<.10$ \\
\hline 360719119205501 & $21 \mathrm{~S} / 24 \mathrm{E}-11$ & $\mathrm{R}$ & $07 / 29 / 02$ & 1420 & $<.10$ & $<.10$ & $<.10$ \\
\hline 360807119114901 & $21 \mathrm{~S} / 26 \mathrm{E}-06$ & $\mathrm{R}$ & 07/30/02 & 1000 & $<.10$ & $<.10$ & $<.10$ \\
\hline 361338119275501 & 019S023E34P002M & $\mathrm{R}$ & 08/08/02 & 1000 & $<.10$ & $<.10$ & $<.10$ \\
\hline 361338119275501 & 019S023E34P002M & $\mathrm{LD}$ & 08/08/02 & 1000 & $<.10$ & $<.10$ & $<.10$ \\
\hline 361341119280102 & 019S023E34P004M & $\mathrm{R}$ & $06 / 18 / 02$ & 1500 & $<.10$ & $<.10$ & $<.10$ \\
\hline 361519119433401 & 019S021E19R001M & $\mathrm{R}$ & $07 / 25 / 02$ & 0950 & $<.10$ & $<.10$ & $<.10$ \\
\hline 361717119234202 & 019S024E08L002M & $\mathrm{R}$ & 06/18/02 & 1040 & $<.10$ & $<.10$ & $<.10$ \\
\hline 361726119241101 & 019S024E07J001M & $\mathrm{R}$ & $07 / 23 / 02$ & 1330 & $<.10$ & $<.10$ & $<.10$ \\
\hline 361852119350601 & 018S022E33R001M & $\mathrm{R}$ & $07 / 31 / 02$ & 1000 & $<.10$ & $<.10$ & $<.10$ \\
\hline 361852119350601 & 018S022E33R001M & $\mathrm{LD}$ & $07 / 31 / 02$ & 1000 & $<.10$ & $<.10$ & $<.10$ \\
\hline 361948119412201 & 018S021E27N003M & $\mathrm{R}$ & 07/26/02 & 1010 & $<.10$ & $<.10$ & $<.10$ \\
\hline 362417119533701 & 017S019E34Q002M & $\mathrm{R}$ & $07 / 17 / 02$ & 1050 & $<.10$ & $<.10$ & $<.10$ \\
\hline 362525119450601 & 017S020E25K001M & $\mathrm{R}$ & $07 / 16 / 02$ & 1220 & $<.10$ & $<.10$ & $<.10$ \\
\hline 362525119450601 & 017S020E25K001M & FR & $07 / 16 / 02$ & 1225 & $<.10$ & $<.10$ & $<.10$ \\
\hline 362537119450902 & 017S020E25G002M & $\mathrm{R}$ & $06 / 19 / 02$ & 1240 & $<.10$ & $<.10$ & $<.10$ \\
\hline 363123119585001 & 016S018E23K001M & $\mathrm{R}$ & 07/17/02 & 1540 & $<.10$ & $<.10$ & $<.10$ \\
\hline
\end{tabular}


Table 5. Analytical results for glyphosate, aminomethylphosphonic acid, and glufosinate in National Water-Quality Assessment (NAWQA) Program surface- and ground-water studies, 2001-06.-Continued

[Location of NAWQA study units shown in figure 4. Sample type: FB, field blank; FR, field replicate; R, regular; LD, laboratory duplicate; --, no data; <, less than; $\mu \mathrm{g} / \mathrm{L}$, micrograms per liter]

\begin{tabular}{|c|c|c|c|c|c|c|c|}
\hline \multirow[b]{2}{*}{$\begin{array}{c}\text { Site } \\
\text { number }\end{array}$} & \multirow[b]{2}{*}{ Site location } & \multirow[b]{2}{*}{$\begin{array}{c}\text { Sample } \\
\text { type }\end{array}$} & \multirow[b]{2}{*}{$\begin{array}{c}\text { Date of } \\
\text { collection } \\
\text { (day/ } \\
\text { month/ } \\
\text { year) }\end{array}$} & \multirow[b]{2}{*}{$\begin{array}{c}\text { Col- } \\
\text { lection } \\
\text { time } \\
(24- \\
\text { hour })\end{array}$} & \multicolumn{3}{|c|}{ Concentrations } \\
\hline & & & & & $\begin{array}{c}\text { Glypho- } \\
\text { sate } \\
(\mu \mathrm{g} / \mathrm{L})\end{array}$ & $\begin{array}{c}\text { Amino- } \\
\text { meth- } \\
\text { ylphos- } \\
\text { phonic } \\
\text { acid } \\
(\mu \mathrm{g} / \mathrm{L})\end{array}$ & $\begin{array}{c}\text { Glufosi- } \\
\text { nate } \\
\text { ( } \mu \text { g/L) }\end{array}$ \\
\hline \multicolumn{8}{|c|}{ San Joaquin-Tulare Basins Ground Water (SAG)_Continued } \\
\hline 363924119530401 & 015S019E03G001M & $\mathrm{R}$ & $07 / 15 / 02$ & 1620 & $<0.10$ & $<0.10$ & $<0.10$ \\
\hline 370046120212001 & 010S015E32P001M & $\mathrm{R}$ & 07/18/02 & 1100 & $<.10$ & $<.10$ & $<.10$ \\
\hline 370618120190101 & 009S015E34K001M & $\mathrm{R}$ & $07 / 18 / 02$ & 1530 & $<.10$ & $<.10$ & $<.10$ \\
\hline 371548120224101 & 008S015E06M001M & $\mathrm{R}$ & $07 / 11 / 02$ & 1230 & $<.10$ & $<.10$ & $<.10$ \\
\hline 371548120224101 & 008S015E06M001M & $\mathrm{LD}$ & 07/11/02 & 1230 & $<.10$ & $<.10$ & $<.10$ \\
\hline 372323120554401 & 006S009E25B001M & $\mathrm{R}$ & 08/06/02 & 1130 & $<.10$ & $<.10$ & $<.10$ \\
\hline 372617120530301 & 006S010E04M001M & $\mathrm{R}$ & 07/11/02 & 1000 & $<.10$ & $<.10$ & $<.10$ \\
\hline 372624120530302 & 006S010E04M003M & $\mathrm{R}$ & 07/22/02 & 1150 & $<.10$ & $<.10$ & $<.10$ \\
\hline 372933120565901 & 005S009E23C002M & $\mathrm{R}$ & 07/10/02 & 1320 & $<.10$ & $<.10$ & $<.10$ \\
\hline 373114120595001 & 005S009E08A001M & $\mathrm{R}$ & 07/09/02 & 1200 & $<.10$ & $<.10$ & $<.10$ \\
\hline 373349121032302 & 004S008E26B002M & $\mathrm{R}$ & 06/20/02 & 1220 & $<.10$ & $<.10$ & $<.10$ \\
\hline 373351121032301 & 004S008E26B003M & $\mathrm{R}$ & 07/08/02 & 1330 & $<.10$ & $<.10$ & $<.10$ \\
\hline 373700121071001 & $4 \mathrm{~S} / 8 \mathrm{E}-5 \mathrm{E} 1$ & $\mathrm{R}$ & 08/29/02 & 0920 & $<.10$ & $<.10$ & $<.10$ \\
\hline 374043121100301 & 003S007E14B001M & $\mathrm{R}$ & 07/10/02 & 1040 & $<.10$ & $<.10$ & $<.10$ \\
\hline 374902121031101 & 001S008E-26 & $\mathrm{R}$ & 09/12/02 & 1030 & $<.10$ & $<.10$ & $<.10$ \\
\hline \multicolumn{8}{|c|}{ South Platte Basin Surface Water (SPL) } \\
\hline \multirow[t]{13}{*}{06713500} & Cherry Creek at Denver, Colorado & $\mathrm{R}$ & $10 / 09 / 02$ & 0959 & $<.10$ & $<.10$ & $<.10$ \\
\hline & & $\mathrm{LD}$ & $10 / 09 / 02$ & 0959 & $<.10$ & $<.10$ & $<.10$ \\
\hline & & FR & $10 / 09 / 02$ & 1000 & $<.10$ & $<.10$ & $<.10$ \\
\hline & & $\mathrm{R}$ & $10 / 09 / 02$ & 1020 & $<.10$ & .30 & $<.10$ \\
\hline & & $\mathrm{R}$ & $11 / 05 / 02$ & 1010 & $<.10$ & .88 & $<.10$ \\
\hline & & $\mathrm{R}$ & $12 / 03 / 02$ & 0945 & $<.10$ & .27 & $<.10$ \\
\hline & & $\mathrm{R}$ & 01/08/03 & 1010 & $<.10$ & .16 & $<.10$ \\
\hline & & $\mathrm{LD}$ & 01/08/03 & 1010 & $<.10$ & .15 & $<.10$ \\
\hline & & FR & 01/08/03 & 1011 & $<.10$ & .20 & $<.10$ \\
\hline & & $\mathrm{R}$ & $02 / 04 / 03$ & 0940 & $<.10$ & .33 & $<.10$ \\
\hline & & $\mathrm{R}$ & 03/05/03 & 1030 & $<.10$ & .23 & $<.10$ \\
\hline & & $\mathrm{R}$ & 04/10/03 & 1010 & $<.10$ & $<.10$ & $<.10$ \\
\hline & & $\mathrm{R}$ & 04/22/03 & 1000 & $<.10$ & $<.10$ & $<.10$ \\
\hline
\end{tabular}


Table 5. Analytical results for glyphosate, aminomethylphosphonic acid, and glufosinate in National Water-Quality Assessment (NAWQA) Program surface- and ground-water studies, 2001-06.-Continued

[Location of NAWQA study units shown in figure 4. Sample type: FB, field blank; FR, field replicate; R, regular; LD, laboratory duplicate; --, no data; <, less than; $\mu \mathrm{g} / \mathrm{L}$, micrograms per liter]

\begin{tabular}{|c|c|c|c|c|c|c|c|}
\hline \multirow[b]{2}{*}{$\begin{array}{c}\text { Site } \\
\text { number }\end{array}$} & \multirow[b]{2}{*}{ Site location } & \multirow[b]{2}{*}{$\begin{array}{c}\text { Sample } \\
\text { type }\end{array}$} & \multirow[b]{2}{*}{$\begin{array}{c}\text { Date of } \\
\text { collection } \\
\text { (day/ } \\
\text { month/ } \\
\text { year) }\end{array}$} & \multirow[b]{2}{*}{$\begin{array}{c}\text { Col- } \\
\text { lection } \\
\text { time } \\
(24- \\
\text { hour) }\end{array}$} & \multicolumn{3}{|c|}{ Concentrations } \\
\hline & & & & & $\begin{array}{c}\text { Glypho- } \\
\text { sate } \\
(\mu \mathrm{g} / \mathrm{L})\end{array}$ & $\begin{array}{c}\text { Amino- } \\
\text { meth- } \\
\text { ylphos- } \\
\text { phonic } \\
\text { acid } \\
\text { ( } \mu \mathrm{g} / \mathrm{L})\end{array}$ & $\begin{array}{c}\text { Glufosi- } \\
\text { nate } \\
\text { ( } \mu \mathrm{g} / \mathrm{L})\end{array}$ \\
\hline \multicolumn{8}{|c|}{ South Platte Basin Surface Water (SPL)_Continued } \\
\hline \multirow[t]{13}{*}{06713500} & Cherry Creek at Denver, Colorado_Continued & FR & $04 / 22 / 03$ & 1020 & $<0.10$ & 0.12 & $<0.10$ \\
\hline & & $\mathrm{R}$ & 05/07/03 & 1000 & $<.10$ & .15 & $<.10$ \\
\hline & & $\mathrm{R}$ & $05 / 20 / 03$ & 1020 & $<.10$ & .14 & $<.10$ \\
\hline & & FR & $05 / 20 / 03$ & 1021 & $<.10$ & .17 & $<.10$ \\
\hline & & $\mathrm{R}$ & $06 / 02 / 03$ & 0845 & .78 & .49 & $<.10$ \\
\hline & & $\mathrm{LD}$ & 06/02/03 & 0845 & .80 & .56 & $<.10$ \\
\hline & & $\mathrm{R}$ & $06 / 23 / 03$ & 0900 & .25 & .31 & $<.10$ \\
\hline & & $\mathrm{LD}$ & $06 / 23 / 03$ & 0900 & .25 & .35 & $<.10$ \\
\hline & & $\mathrm{R}$ & 07/08/03 & 1000 & $<.10$ & .14 & $<.10$ \\
\hline & & $\mathrm{R}$ & $08 / 05 / 03$ & 1110 & .43 & .37 & $<.10$ \\
\hline & & $\mathrm{R}$ & $08 / 15 / 03$ & 1030 & .30 & .49 & $<.10$ \\
\hline & & $\mathrm{R}$ & 09/11/03 & 1030 & $<.10$ & .29 & $<.10$ \\
\hline & & $\mathrm{R}$ & 09/22/03 & 1030 & $<.10$ & .14 & $<.10$ \\
\hline \multicolumn{8}{|c|}{ Trinity River Basin Ground Water (TRG) } \\
\hline \multirow[t]{3}{*}{294503095373201} & MW29 & $\mathrm{R}$ & $12 / 14 / 03$ & 0958 & $<.10$ & $<.10$ & $<.10$ \\
\hline & & $\mathrm{LD}$ & $12 / 14 / 03$ & 0958 & $<.10$ & $<.10$ & $<.10$ \\
\hline & & FR & $12 / 14 / 03$ & 1001 & $<.10$ & $<.10$ & $<.10$ \\
\hline 294620095440501 & MW17 & $\mathrm{R}$ & $12 / 15 / 03$ & 1001 & $<.10$ & $<.10$ & $<.10$ \\
\hline 294405095412301 & REF01 & $\mathrm{R}$ & $12 / 15 / 03$ & 1401 & $<.10$ & $<.10$ & $<.10$ \\
\hline \multirow[t]{2}{*}{294957095310801} & MW26 & $\mathrm{R}$ & $12 / 12 / 03$ & 1101 & $<.10$ & $<.10$ & $<.10$ \\
\hline & & FR & $12 / 12 / 03$ & 1103 & $<.10$ & $<.10$ & $<.10$ \\
\hline 294919095320501 & MW25 & $\mathrm{R}$ & $12 / 13 / 03$ & 0931 & $<.10$ & $<.10$ & $<.10$ \\
\hline 294807095452701 & MW27 & $\mathrm{R}$ & $12 / 13 / 03$ & 1401 & $<.10$ & $<.10$ & $<.10$ \\
\hline 294800095415801 & MW28A & $\mathrm{R}$ & $12 / 21 / 03$ & 1701 & $<.10$ & $<.10$ & $<.10$ \\
\hline \multirow[t]{2}{*}{295049095253101} & MW23 & $\mathrm{R}$ & $12 / 10 / 03$ & 1001 & $<.10$ & $<.10$ & $<.10$ \\
\hline & & $\mathrm{LD}$ & $12 / 10 / 03$ & 1001 & $<.10$ & $<.10$ & $<.10$ \\
\hline 295133095273201 & MW22 & $\mathrm{R}$ & $12 / 11 / 03$ & 1001 & $<.10$ & $<.10$ & $<.10$ \\
\hline 295150095302401 & MW20 & $\mathrm{R}$ & $12 / 12 / 03$ & 1358 & $<.10$ & $<.10$ & $<.10$ \\
\hline
\end{tabular}


Table 5. Analytical results for glyphosate, aminomethylphosphonic acid, and glufosinate in National Water-Quality Assessment (NAWQA) Program surface- and ground-water studies, 2001-06.-Continued

[Location of NAWQA study units shown in figure 4. Sample type: FB, field blank; FR, field replicate; R, regular; LD, laboratory duplicate; --, no data; <, less than; $\mu \mathrm{g} / \mathrm{L}$, micrograms per liter]

\begin{tabular}{|c|c|c|c|c|c|c|c|}
\hline \multirow[b]{2}{*}{$\begin{array}{c}\text { Site } \\
\text { number }\end{array}$} & & \multirow[b]{2}{*}{$\begin{array}{c}\text { Sample } \\
\text { type }\end{array}$} & \multirow[b]{2}{*}{$\begin{array}{c}\text { Date of } \\
\text { collection } \\
\text { (day/ } \\
\text { month/ } \\
\text { year) }\end{array}$} & \multirow[b]{2}{*}{$\begin{array}{l}\text { Col- } \\
\text { lection } \\
\text { time } \\
\text { (24- } \\
\text { hour) }\end{array}$} & \multicolumn{3}{|c|}{ Concentrations } \\
\hline & & & & & $\begin{array}{c}\text { Glypho- } \\
\text { sate } \\
(\mu \mathrm{g} / \mathrm{L})\end{array}$ & $\begin{array}{c}\text { Amino- } \\
\text { meth- } \\
\text { ylphos- } \\
\text { phonic } \\
\text { acid } \\
(\mu \mathrm{g} / \mathrm{L}) \\
\end{array}$ & $\begin{array}{c}\text { Glufosi- } \\
\text { nate } \\
(\mu \mathrm{g} / \mathrm{L})\end{array}$ \\
\hline \multicolumn{8}{|c|}{ Trinity River Basin Ground Water (TRG)—Continued } \\
\hline 295150095302401 & MW20 & FR & $12 / 12 / 03$ & 1401 & $<0.10$ & $<0.10$ & $<0.10$ \\
\hline 295232095294101 & MW21 & $\mathrm{R}$ & $12 / 11 / 03$ & 1401 & $<.10$ & $<.10$ & $<.10$ \\
\hline 295249095411301 & MW16 & $\mathrm{R}$ & $12 / 16 / 03$ & 1101 & $<.10$ & $<.10$ & $<.10$ \\
\hline 295358095374101 & MW18 & $\mathrm{R}$ & $12 / 18 / 03$ & 1101 & $<.10$ & $<.10$ & $<.10$ \\
\hline 295421095305801 & MW19 & $\mathrm{R}$ & $12 / 19 / 03$ & 1001 & $<.10$ & $<.10$ & $<.10$ \\
\hline 295557095360901 & MW11 & $\mathrm{R}$ & $12 / 16 / 03$ & 1401 & $<.10$ & $<.10$ & $<.10$ \\
\hline 295633095335201 & MW12 & $\mathrm{R}$ & $12 / 17 / 03$ & 1101 & $<.10$ & $<.10$ & $<.10$ \\
\hline \multirow[t]{2}{*}{295711095222301} & MW07 & $\mathrm{R}$ & $12 / 17 / 03$ & 1101 & $<.10$ & $<.10$ & $<.10$ \\
\hline & & $\mathrm{LD}$ & $12 / 17 / 03$ & 1101 & $<.10$ & $<.10$ & $<.10$ \\
\hline 295720095290001 & MW08 & $\mathrm{R}$ & $12 / 17 / 03$ & 1601 & $<.10$ & $<.10$ & $<.10$ \\
\hline 300011095251801 & MW10 & $\mathrm{R}$ & $12 / 17 / 03$ & 1601 & $<.10$ & $<.10$ & $<.10$ \\
\hline 300026095225401 & MW06 & $\mathrm{R}$ & $12 / 19 / 03$ & 1001 & $<.10$ & $<.10$ & $<.10$ \\
\hline 300036095400101 & REF02 & $\mathrm{R}$ & $12 / 29 / 03$ & 1101 & $<.10$ & $<.10$ & $<.10$ \\
\hline 300044095312001 & MW01 & $\mathrm{R}$ & $12 / 19 / 03$ & 1501 & $<.10$ & $<.10$ & $<.10$ \\
\hline \multirow[t]{2}{*}{300155095200201} & MW05 & $\mathrm{R}$ & $12 / 20 / 03$ & 1101 & $<.10$ & $<.10$ & $<.10$ \\
\hline & & FR & $12 / 20 / 03$ & 1103 & $<.10$ & $<.10$ & $<.10$ \\
\hline 300333095291701 & MW03 & $\mathrm{R}$ & $12 / 29 / 03$ & 1701 & $<.10$ & $<.10$ & $<.10$ \\
\hline 300351095232601 & MW02 & $\mathrm{R}$ & $12 / 18 / 03$ & 1201 & $<.10$ & $<.10$ & $<.10$ \\
\hline 300825095274801 & MW14 & $\mathrm{R}$ & $12 / 30 / 03$ & 1701 & $<.10$ & $<.10$ & $<.10$ \\
\hline \multirow[t]{2}{*}{301008095302901} & MW13 & $\mathrm{R}$ & $12 / 22 / 03$ & 1801 & $<.10$ & $<.10$ & $<.10$ \\
\hline & & $\mathrm{LD}$ & $12 / 22 / 03$ & 1801 & $<.10$ & $<.10$ & $<.10$ \\
\hline \multirow[t]{2}{*}{301220095305501} & MW15 & $\mathrm{R}$ & $12 / 22 / 03$ & 1101 & $<.10$ & $<.10$ & $<.10$ \\
\hline & & LD & $12 / 22 / 03$ & 1101 & $<.10$ & $<.10$ & $<.10$ \\
\hline 301716095400501 & REF03 & $\mathrm{R}$ & $12 / 30 / 03$ & 1001 & $<.10$ & $<.10$ & $<.10$ \\
\hline \multicolumn{8}{|c|}{ Upper Snake River Basin Ground Water (USG) } \\
\hline 423723113495801 & 09S 22E 23DAD1 & $\mathrm{R}$ & $06 / 21 / 05$ & 0732 & $<.02$ & $<.02$ & $<.02$ \\
\hline 423805113465501 & 09S 23E 16CCC1 & $\mathrm{R}$ & $06 / 21 / 05$ & 1000 & $<.02$ & $<.02$ & $<.02$ \\
\hline 423857113493301 & 09S 22E 12 CDC1 & $\mathrm{R}$ & $06 / 21 / 05$ & 1200 & $<.02$ & $<.02$ & $<.02$ \\
\hline 423934113430001 & 09S 23E 12BAB1 & $\mathrm{R}$ & $06 / 21 / 05$ & 1330 & $<.02$ & $<.02$ & $<.02$ \\
\hline 424226113454901 & 08S 23E 21DDD1 & $\mathrm{R}$ & 06/22/05 & 0800 & $<.02$ & $<.02$ & $<.02$ \\
\hline
\end{tabular}


Table 5. Analytical results for glyphosate, aminomethylphosphonic acid, and glufosinate in National Water-Quality Assessment (NAWQA) Program surface- and ground-water studies, 2001-06.-Continued

[Location of NAWQA study units shown in figure 4. Sample type: FB, field blank; FR, field replicate; R, regular; LD, laboratory duplicate; --, no data; <, less than; $\mu \mathrm{g} / \mathrm{L}$, micrograms per liter]

\begin{tabular}{|c|c|c|c|c|c|c|c|}
\hline \multirow[b]{2}{*}{$\begin{array}{c}\text { Site } \\
\text { number }\end{array}$} & \multirow[b]{2}{*}{ Site location } & \multirow[b]{2}{*}{$\begin{array}{c}\text { Sample } \\
\text { type }\end{array}$} & \multirow[b]{2}{*}{$\begin{array}{l}\text { Date of } \\
\text { collection } \\
\text { (day/ } \\
\text { month/ } \\
\text { year) }\end{array}$} & \multirow[b]{2}{*}{$\begin{array}{l}\text { Col- } \\
\text { lection } \\
\text { time } \\
(24- \\
\text { hour })\end{array}$} & \multicolumn{3}{|c|}{ Concentrations } \\
\hline & & & & & $\begin{array}{c}\text { Glypho- } \\
\text { sate } \\
(\mu \mathrm{g} / \mathrm{L})\end{array}$ & $\begin{array}{c}\text { Amino- } \\
\text { meth- } \\
\text { ylphos- } \\
\text { phonic } \\
\text { acid } \\
\text { ( } \mu \mathrm{g} / \mathrm{L})\end{array}$ & $\begin{array}{c}\text { Glufosi- } \\
\text { nate } \\
(\mu \mathrm{g} / \mathrm{L})\end{array}$ \\
\hline \multicolumn{8}{|c|}{ Upper Snake River Basin Ground Water (USG) —Continued } \\
\hline 424159113432801 & 08S 23E 26 ADD2 & $\mathrm{R}$ & $06 / 22 / 05$ & 1000 & $<0.02$ & $<0.02$ & $<0.02$ \\
\hline 424133113432401 & $08 \mathrm{~S} 23 \mathrm{E} 25 \mathrm{CCC} 1$ & $\mathrm{R}$ & $06 / 22 / 05$ & 1130 & $<.02$ & $<.02$ & $<.02$ \\
\hline 424355113503201 & $08 \mathrm{~S} 22 \mathrm{E} 14 \mathrm{ABCC} 1$ & $\mathrm{R}$ & $06 / 23 / 05$ & 0800 & $<.02$ & $<.02$ & $<.02$ \\
\hline 424352113235601 & 08S 26E 15ABD1 & $\mathrm{R}$ & $06 / 27 / 05$ & 1200 & $<.02$ & $<.02$ & $<.02$ \\
\hline 424421113293201 & 08S 25E 11DAD1 & $\mathrm{R}$ & $06 / 27 / 05$ & 1400 & $<.02$ & $<.02$ & $<.02$ \\
\hline 424448113332501 & 08S 25E 8ABA1 & $\mathrm{R}$ & $06 / 28 / 05$ & 0800 & $<.02$ & $<.02$ & $<.02$ \\
\hline 424544113303201 & 08S 25E 2BBB1 & $\mathrm{R}$ & $06 / 28 / 05$ & 1000 & $<.02$ & $<.02$ & $<.02$ \\
\hline 424333113363301 & 08S 24E 14DAD1 & $\mathrm{R}$ & 06/28/05 & 1200 & $<.02$ & $<.02$ & $<.02$ \\
\hline 424629113385201 & 07S 24E 33ADA1 & $\mathrm{R}$ & 06/29/05 & 0800 & $<.02$ & $<.02$ & $<.02$ \\
\hline 424550113400301 & 08S 24E 05AAA2 & $\mathrm{R}$ & 06/29/05 & 0930 & $<.02$ & $<.02$ & $<.02$ \\
\hline 424647113444201 & 07S 23E 27DDD1 & $\mathrm{R}$ & 06/29/05 & 1200 & $<.02$ & $<.02$ & $<.02$ \\
\hline 424227113381301 & 08S 24E 22DCC1 & $\mathrm{R}$ & 06/30/05 & 0730 & $<.02$ & $<.02$ & $<.02$ \\
\hline 424041113392501 & 08S 24E 33DCC1 & $\mathrm{R}$ & 06/30/05 & 1000 & $<.02$ & $<.02$ & $<.02$ \\
\hline \multirow[t]{3}{*}{424220113362701} & 08S 24E 25BBB1 & $\mathrm{R}$ & 06/30/05 & 1200 & $<.02$ & $<.02$ & $<.02$ \\
\hline & & FR & 06/30/05 & 1208 & $<.02$ & $<.02$ & $<.02$ \\
\hline & & FR & 06/30/05 & 1210 & $<.02$ & $<.02$ & $<.02$ \\
\hline 424629113385201 & 07S 24E 33ADA1 & $\mathrm{R}$ & 06/29/05 & 0800 & $<.02$ & $<.02$ & $<.02$ \\
\hline 424448113332501 & 08S 25E 8ABA1 & $\mathrm{R}$ & 06/28/05 & 0800 & $<.02$ & $<.02$ & $<.02$ \\
\hline 424220113362701 & 08S 24E 25BBB1 & $\mathrm{R}$ & 06/30/05 & 1210 & $<.02$ & $<.02$ & $<.02$ \\
\hline 424617113421901 & 07S 23E 36DAA1 & $\mathrm{R}$ & 07/20/05 & 1000 & $<.02$ & $<.02$ & $<.02$ \\
\hline 424407113412001 & 08S 24E 18AAA1 & $\mathrm{R}$ & 07/20/05 & 1230 & $<.02$ & $<.02$ & $<.02$ \\
\hline 424555113463901 & $07 \mathrm{~S} 23 \mathrm{E} 33 \mathrm{CDC} 1$ & $\mathrm{R}$ & 07/20/05 & 1600 & $<.02$ & $<.02$ & $<.02$ \\
\hline 424146113402201 & 08S 24E 29DBD1 & $\mathrm{R}$ & $07 / 21 / 05$ & 0930 & $<.02$ & $<.02$ & $<.02$ \\
\hline 424609113312001 & 07S 25E 34CAD1 & $\mathrm{R}$ & 07/21/05 & 1230 & $<.02$ & $<.02$ & $<.02$ \\
\hline 424251113443401 & $08 \mathrm{~S} 23 \mathrm{E} 23 \mathrm{BCC} 1$ & $\mathrm{R}$ & 07/21/05 & 1500 & $<.02$ & $<.02$ & $<.02$ \\
\hline 423857113473301 & 09S 23E 08CDD1 & $\mathrm{R}$ & 07/25/05 & 1200 & $<.02$ & 0.02 & $<.02$ \\
\hline 423857113532701 & 09S 22E 09CCC1 & $\mathrm{R}$ & $07 / 25 / 05$ & 1445 & $<.02$ & $<.02$ & $<.02$ \\
\hline 423806113543701 & 09S 22E 17CCC1 & $\mathrm{R}$ & $07 / 25 / 05$ & 1700 & $<.02$ & $<.02$ & $<.02$ \\
\hline
\end{tabular}


Table 5. Analytical results for glyphosate, aminomethylphosphonic acid, and glufosinate in National Water-Quality Assessment (NAWQA) Program surface- and ground-water studies, 2001-06.-Continued

[Location of NAWQA study units shown in figure 4. Sample type: FB, field blank; FR, field replicate; R, regular; LD, laboratory duplicate; --, no data; <, less than; $\mu \mathrm{g} / \mathrm{L}$, micrograms per liter]

\begin{tabular}{|c|c|c|c|c|c|c|c|}
\hline \multirow{2}{*}{$\begin{array}{c}\text { Site } \\
\text { number }\end{array}$} & \multirow[b]{2}{*}{ Site location } & \multirow[b]{2}{*}{$\begin{array}{c}\text { Sample } \\
\text { type }\end{array}$} & \multirow[b]{2}{*}{$\begin{array}{c}\text { Date of } \\
\text { collection } \\
\text { (day/ } \\
\text { month/ } \\
\text { year) }\end{array}$} & \multirow[b]{2}{*}{$\begin{array}{c}\text { Col- } \\
\text { lection } \\
\text { time } \\
\text { (24- } \\
\text { hour) }\end{array}$} & \multicolumn{3}{|c|}{ Concentrations } \\
\hline & & & & & $\begin{array}{c}\text { Glypho- } \\
\text { sate } \\
\text { ( } \mathrm{gg} / \mathrm{L})\end{array}$ & $\begin{array}{c}\text { Amino- } \\
\text { meth- } \\
\text { ylphos- } \\
\text { phonic } \\
\text { acid } \\
(\mu \mathrm{g} / \mathrm{L}) \\
\end{array}$ & $\begin{array}{c}\text { Glufosi- } \\
\text { nate } \\
(\mu \mathrm{g} / \mathrm{L})\end{array}$ \\
\hline \multicolumn{8}{|c|}{ Upper Snake River Basin Ground Water (USG)_Continued } \\
\hline 424317113485101 & 08S 22E 13DDC1 & $\mathrm{R}$ & $07 / 26 / 05$ & 1015 & $<0.02$ & $<0.02$ & $<0.02$ \\
\hline 423948113522201 & 09S 22E 04DDD1 & $\mathrm{R}$ & 07/26/05 & 1200 & $<.02$ & $<.02$ & $<.02$ \\
\hline 423948113522201 & 09S 22E 04DDD1 & FR & 07/26/05 & 1208 & $<.02$ & $<.02$ & $<.02$ \\
\hline 423948113522201 & 09S 22E 04DDD1 & FR & 07/26/05 & 1212 & $<.02$ & $<.02$ & $<.02$ \\
\hline 424132113322102 & $8 \mathrm{~S} 25 \mathrm{E} 28 \mathrm{DCC} 2$ & $\mathrm{R}$ & 08/31/05 & 1645 & $<.02$ & $<.02$ & $<.02$ \\
\hline \multicolumn{8}{|c|}{ White River Basin Surface Water (WHM) } \\
\hline \multirow[t]{21}{*}{03267900} & Mad River at St. Paris Pike at Eagle City, Ohio & $\mathrm{R}$ & $10 / 02 / 02$ & 1100 & $<.10$ & $<.10$ & $<.10$ \\
\hline & & $\mathrm{R}$ & $11 / 06 / 02$ & 1030 & $<.10$ & $<.10$ & $<.10$ \\
\hline & & $\mathrm{R}$ & $12 / 04 / 02$ & 1130 & $<.10$ & $<.10$ & $<.10$ \\
\hline & & $\mathrm{R}$ & $12 / 05 / 02$ & 1330 & $<.10$ & $<.10$ & $<.10$ \\
\hline & & $\mathrm{R}$ & $12 / 17 / 02$ & 1300 & $<.10$ & $<.10$ & $<.10$ \\
\hline & & $\mathrm{R}$ & $02 / 12 / 03$ & 1130 & $<.10$ & $<.10$ & $<.10$ \\
\hline & & $\mathrm{R}$ & 03/13/03 & 1115 & $<.10$ & $<.10$ & $<.10$ \\
\hline & & $\mathrm{R}$ & $04 / 08 / 03$ & 1300 & $<.10$ & $<.10$ & $<.10$ \\
\hline & & $\mathrm{R}$ & $04 / 22 / 03$ & 1200 & $<.10$ & $<.10$ & $<.10$ \\
\hline & & $\mathrm{R}$ & 05/13/03 & 1200 & $<.10$ & $<.10$ & $<.10$ \\
\hline & & $\mathrm{R}$ & 05/28/03 & 1330 & $<.10$ & .11 & $<.10$ \\
\hline & & $\mathrm{R}$ & 06/11/03 & 1345 & $<.10$ & $<.10$ & $<.10$ \\
\hline & & $\mathrm{R}$ & 06/25/03 & 1200 & $<.10$ & $<.10$ & $<.10$ \\
\hline & & $\mathrm{R}$ & 07/08/03 & 1016 & .50 & .94 & $<.10$ \\
\hline & & FB & 07/08/03 & 1015 & $<.10$ & $<.10$ & $<.10$ \\
\hline & & LD & 07/08/03 & 1015 & $<.10$ & $<.10$ & $<.10$ \\
\hline & & $\mathrm{R}$ & 07/30/03 & 1100 & $<.10$ & $<.10$ & $<.10$ \\
\hline & & $\mathrm{R}$ & 08/06/03 & 1114 & $<.10$ & $<.10$ & $<.10$ \\
\hline & & $\mathrm{R}$ & 08/06/03 & 1115 & $<.10$ & .22 & $<.10$ \\
\hline & & $\mathrm{R}$ & 08/26/03 & 1045 & $<.10$ & $<.10$ & $<.10$ \\
\hline & & LD & 08/26/03 & 1045 & $<.10$ & $<.10$ & $<.10$ \\
\hline
\end{tabular}


Table 5. Analytical results for glyphosate, aminomethylphosphonic acid, and glufosinate in National Water-Quality Assessment (NAWQA) Program surface- and ground-water studies, 2001-06.-Continued

[Location of NAWQA study units shown in figure 4. Sample type: FB, field blank; FR, field replicate; R, regular; LD, laboratory duplicate; --, no data; <, less than; $\mu \mathrm{g} / \mathrm{L}$, micrograms per liter]

\begin{tabular}{|c|c|c|c|c|c|c|c|}
\hline \multirow[b]{2}{*}{$\begin{array}{c}\text { Site } \\
\text { number }\end{array}$} & \multirow[b]{2}{*}{ Site location } & \multirow[b]{2}{*}{$\begin{array}{l}\text { Sample } \\
\text { type }\end{array}$} & \multirow[b]{2}{*}{$\begin{array}{c}\text { Date of } \\
\text { collection } \\
\text { (day/ } \\
\text { month/ } \\
\text { year) }\end{array}$} & \multirow[b]{2}{*}{$\begin{array}{l}\text { Col- } \\
\text { lection } \\
\text { time } \\
(24- \\
\text { hour })\end{array}$} & \multicolumn{3}{|c|}{ Concentrations } \\
\hline & & & & & $\begin{array}{c}\text { Glypho- } \\
\text { sate } \\
(\mu \mathrm{g} / \mathrm{L})\end{array}$ & $\begin{array}{c}\text { Amino- } \\
\text { meth- } \\
\text { ylphos- } \\
\text { phonic } \\
\text { acid } \\
\text { ( } \mu \mathrm{g} / \mathrm{L})\end{array}$ & $\begin{array}{c}\text { Glufosi- } \\
\text { nate } \\
\text { ( } \mu \mathrm{g} / \mathrm{L})\end{array}$ \\
\hline \multicolumn{8}{|c|}{ White River Basin Surface Water (WHM)—Continued } \\
\hline \multirow[t]{2}{*}{03267900} & \multirow{2}{*}{$\begin{array}{l}\text { Mad River at St. Paris Pike at Eagle City, } \\
\text { Ohio-Continued }\end{array}$} & $\mathrm{R}$ & $09 / 04 / 03$ & 1130 & $<0.10$ & 0.13 & $<0.10$ \\
\hline & & $\mathrm{R}$ & $01 / 22 / 03$ & 1115 & $<.10$ & .13 & $<.10$ \\
\hline \multirow[t]{22}{*}{03353637} & \multirow[t]{22}{*}{ Little Buck Creek near Indianapolis, Indiana } & $\mathrm{R}$ & $10 / 08 / 02$ & 1130 & $<.10$ & .31 & $<.10$ \\
\hline & & $\mathrm{R}$ & $11 / 06 / 02$ & 1230 & $<.10$ & $<.10$ & $<.10$ \\
\hline & & $\mathrm{R}$ & $11 / 27 / 02$ & 1330 & $<.10$ & $<.10$ & $<.10$ \\
\hline & & $\mathrm{R}$ & $12 / 09 / 02$ & 1230 & $<.10$ & $<.10$ & $<.10$ \\
\hline & & $\mathrm{R}$ & $01 / 15 / 03$ & 1110 & $<.10$ & $<.10$ & $<.10$ \\
\hline & & $\mathrm{R}$ & $02 / 13 / 03$ & 1130 & $<.10$ & $<.10$ & $<.10$ \\
\hline & & $\mathrm{R}$ & 03/17/03 & 1040 & .12 & $<.10$ & $<.10$ \\
\hline & & $\mathrm{R}$ & $04 / 09 / 03$ & 1150 & $<.10$ & $<.10$ & $<.10$ \\
\hline & & $\mathrm{R}$ & $04 / 22 / 03$ & 1210 & $<.10$ & $<.10$ & $<.10$ \\
\hline & & $\mathrm{LD}$ & $04 / 22 / 03$ & 1210 & $<.10$ & $<.10$ & $<.10$ \\
\hline & & $\mathrm{R}$ & 05/05/03 & 1120 & .21 & $<.10$ & $<.10$ \\
\hline & & $\mathrm{R}$ & 05/20/03 & 1040 & $<.10$ & $<.10$ & $<.10$ \\
\hline & & $\mathrm{R}$ & 06/02/03 & 1328 & $<.10$ & $<.10$ & $<.10$ \\
\hline & & FR & 06/02/03 & 1329 & $<.10$ & $<.10$ & $<.10$ \\
\hline & & $\mathrm{FB}$ & 06/02/03 & 1330 & $<.10$ & $<.10$ & $<.10$ \\
\hline & & $\mathrm{R}$ & $06 / 25 / 03$ & 1110 & $<.10$ & $<.10$ & $<.10$ \\
\hline & & $\mathrm{R}$ & 07/08/03 & 1040 & $<.10$ & .18 & $<.10$ \\
\hline & & $\mathrm{R}$ & 07/21/03 & 1210 & 1.1 & .41 & $<.10$ \\
\hline & & FR & 07/21/03 & 1211 & .73 & .49 & $<.10$ \\
\hline & & $\mathrm{R}$ & 08/05/03 & 1258 & $<.10$ & $<.10$ & $<.10$ \\
\hline & & $\mathrm{R}$ & 08/05/03 & 1300 & .35 & .35 & .11 \\
\hline & & $\mathrm{R}$ & 09/02/03 & 1130 & .16 & .10 & $<.10$ \\
\hline \multirow[t]{2}{*}{03374100} & \multirow[t]{2}{*}{ White River at Hazelton, Indiana } & $\mathrm{R}$ & $10 / 15 / 02$ & 1500 & $<.10$ & .60 & $<.10$ \\
\hline & & $\mathrm{R}$ & $11 / 12 / 02$ & 1340 & $<.10$ & .14 & $<.10$ \\
\hline
\end{tabular}


Table 5. Analytical results for glyphosate, aminomethylphosphonic acid, and glufosinate in National Water-Quality Assessment (NAWQA) Program surface- and ground-water studies, 2001-06.-Continued

[Location of NAWQA study units shown in figure 4. Sample type: FB, field blank; FR, field replicate; R, regular; LD, laboratory duplicate; --, no data; <, less than; $\mu \mathrm{g} / \mathrm{L}$, micrograms per liter]

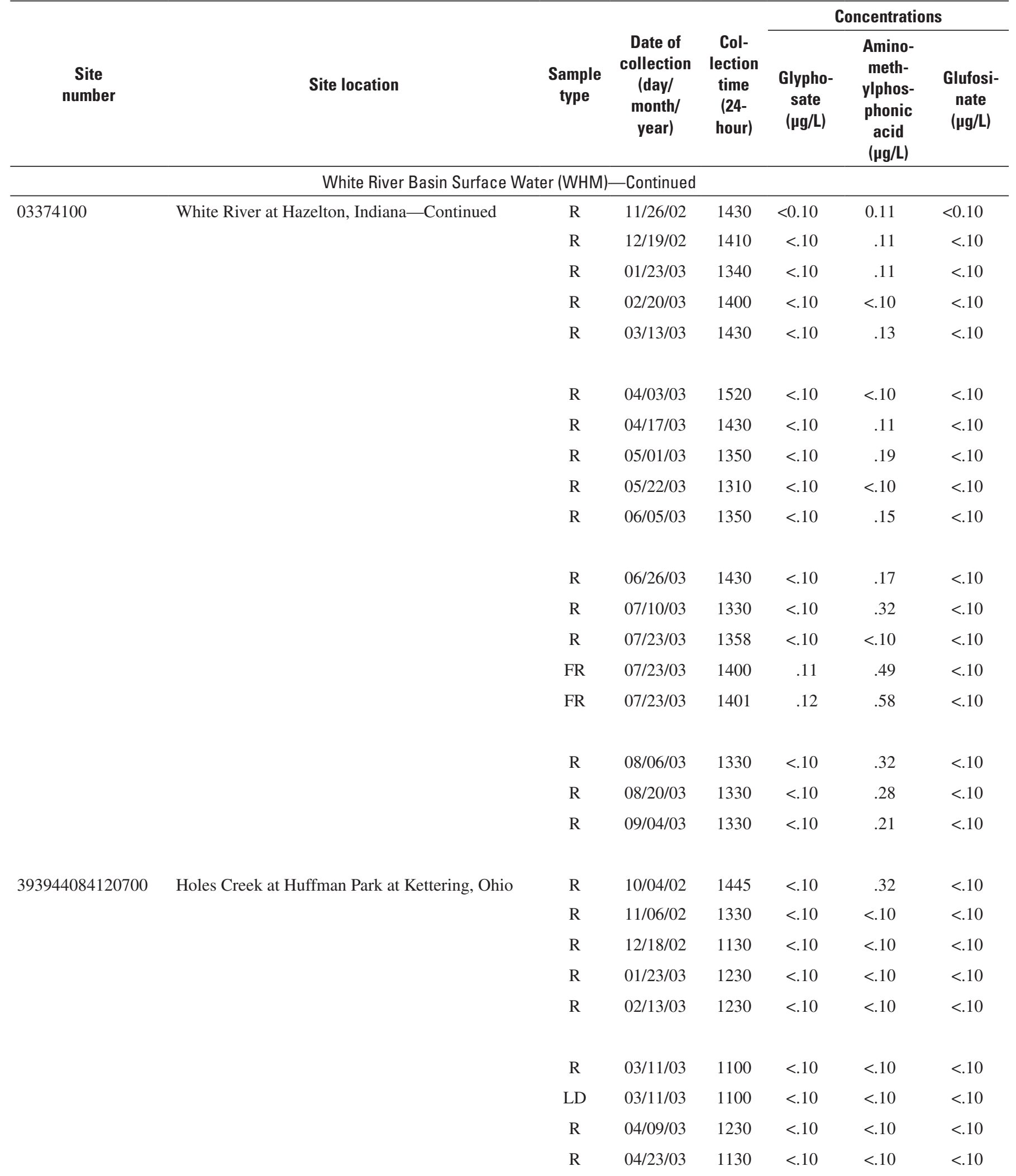


Table 5. Analytical results for glyphosate, aminomethylphosphonic acid, and glufosinate in National Water-Quality Assessment (NAWQA) Program surface- and ground-water studies, 2001-06.-Continued

[Location of NAWQA study units shown in figure 4. Sample type: FB, field blank; FR, field replicate; R, regular; LD, laboratory duplicate; --, no data; <, less than; $\mu \mathrm{g} / \mathrm{L}$, micrograms per liter]

\begin{tabular}{|c|c|c|c|c|c|c|c|}
\hline \multirow[b]{2}{*}{$\begin{array}{c}\text { Site } \\
\text { number }\end{array}$} & \multirow[b]{2}{*}{ Site location } & \multirow[b]{2}{*}{$\begin{array}{c}\text { Sample } \\
\text { type }\end{array}$} & \multirow[b]{2}{*}{$\begin{array}{c}\text { Date of } \\
\text { collection } \\
\text { (day/ } \\
\text { month/ } \\
\text { year) }\end{array}$} & \multirow[b]{2}{*}{$\begin{array}{l}\text { Col- } \\
\text { lection } \\
\text { time } \\
(24- \\
\text { hour })\end{array}$} & \multicolumn{3}{|c|}{ Concentrations } \\
\hline & & & & & $\begin{array}{c}\text { Glypho- } \\
\text { sate } \\
(\mu \mathrm{g} / \mathrm{L})\end{array}$ & $\begin{array}{c}\text { Amino- } \\
\text { meth- } \\
\text { ylphos- } \\
\text { phonic } \\
\text { acid } \\
(\mu \mathrm{g} / \mathrm{L})\end{array}$ & $\begin{array}{c}\text { Glufosi- } \\
\text { nate } \\
\text { ( } \mu \mathrm{g} / \mathrm{L})\end{array}$ \\
\hline \multicolumn{8}{|c|}{ White River Basin Surface Water (WHM)—Continued } \\
\hline \multirow[t]{13}{*}{393944084120700} & \multirow{13}{*}{$\begin{array}{l}\text { Holes Creek at Huffman Park at Kettering, } \\
\text { Ohio-Continued }\end{array}$} & $\mathrm{R}$ & $05 / 28 / 03$ & 1130 & 0.23 & $<0.10$ & $<0.10$ \\
\hline & & $\mathrm{R}$ & $06 / 11 / 03$ & 1100 & .67 & .25 & $<.10$ \\
\hline & & $\mathrm{R}$ & $06 / 26 / 03$ & 1200 & $<.10$ & $<.10$ & $<.10$ \\
\hline & & FR & $06 / 26 / 03$ & 1201 & $<.10$ & $<.10$ & $<.10$ \\
\hline & & $\mathrm{R}$ & 07/10/03 & 1200 & $<.10$ & .19 & $<.10$ \\
\hline & & FR & 07/10/03 & 1201 & $<.10$ & $<.10$ & $<.10$ \\
\hline & & $\mathrm{R}$ & $07 / 31 / 03$ & 1230 & $<.10$ & .14 & $<.10$ \\
\hline & & $\mathrm{R}$ & 08/05/03 & 1229 & $<.10$ & $<.10$ & $<.10$ \\
\hline & & FR & $08 / 05 / 03$ & 1230 & $<.10$ & .27 & $<.10$ \\
\hline & & $\mathrm{R}$ & $08 / 27 / 03$ & 1315 & 1.2 & .15 & $<.10$ \\
\hline & & $\mathrm{R}$ & 09/03/03 & 1130 & .36 & .24 & $<.10$ \\
\hline & & $\mathrm{LD}$ & 09/03/03 & 1130 & .30 & .31 & $<.10$ \\
\hline & & $\mathrm{R}$ & $05 / 14 / 03$ & 1100 & $<.10$ & .10 & $<.10$ \\
\hline \multirow[t]{13}{*}{394340085524601} & \multirow{13}{*}{$\begin{array}{l}\text { Sugar Creek at County Road } 400 \text { South, New } \\
\text { Palestine, Indiana }\end{array}$} & $\mathrm{R}$ & $10 / 09 / 02$ & 1310 & $<.10$ & .38 & $<.10$ \\
\hline & & $\mathrm{R}$ & $11 / 05 / 02$ & 1330 & $<.10$ & $<.10$ & $<.10$ \\
\hline & & $\mathrm{R}$ & $11 / 25 / 02$ & 1330 & $<.10$ & $<.10$ & $<.10$ \\
\hline & & $\mathrm{R}$ & $12 / 16 / 02$ & 1100 & $<.10$ & $<.10$ & $<.10$ \\
\hline & & $\mathrm{R}$ & $01 / 16 / 03$ & 1100 & $<.10$ & $<.10$ & $<.10$ \\
\hline & & $\mathrm{R}$ & $02 / 18 / 03$ & 1130 & $<.10$ & $<.10$ & $<.10$ \\
\hline & & $\mathrm{R}$ & $03 / 11 / 03$ & 1200 & $<.10$ & .26 & $<.10$ \\
\hline & & $\mathrm{R}$ & $04 / 01 / 03$ & 1140 & $<.10$ & $<.10$ & $<.10$ \\
\hline & & $\mathrm{R}$ & $04 / 15 / 03$ & 1130 & $<.10$ & $<.10$ & $<.10$ \\
\hline & & $\mathrm{R}$ & 05/06/03 & 1340 & .18 & .11 & $<.10$ \\
\hline & & $\mathrm{R}$ & 05/06/03 & 1341 & .16 & .13 & $<.10$ \\
\hline & & $\mathrm{R}$ & $05 / 21 / 03$ & 1100 & $<.10$ & $<.10$ & $<.10$ \\
\hline & & LD & $05 / 21 / 03$ & 1100 & $<.10$ & $<.10$ & $<.10$ \\
\hline
\end{tabular}


Table 5. Analytical results for glyphosate, aminomethylphosphonic acid, and glufosinate in National Water-Quality Assessment (NAWQA) Program surface- and ground-water studies, 2001-06.-Continued

[Location of NAWQA study units shown in figure 4. Sample type: FB, field blank; FR, field replicate; R, regular; LD, laboratory duplicate; --, no data; <, less than; $\mu \mathrm{g} / \mathrm{L}$, micrograms per liter]

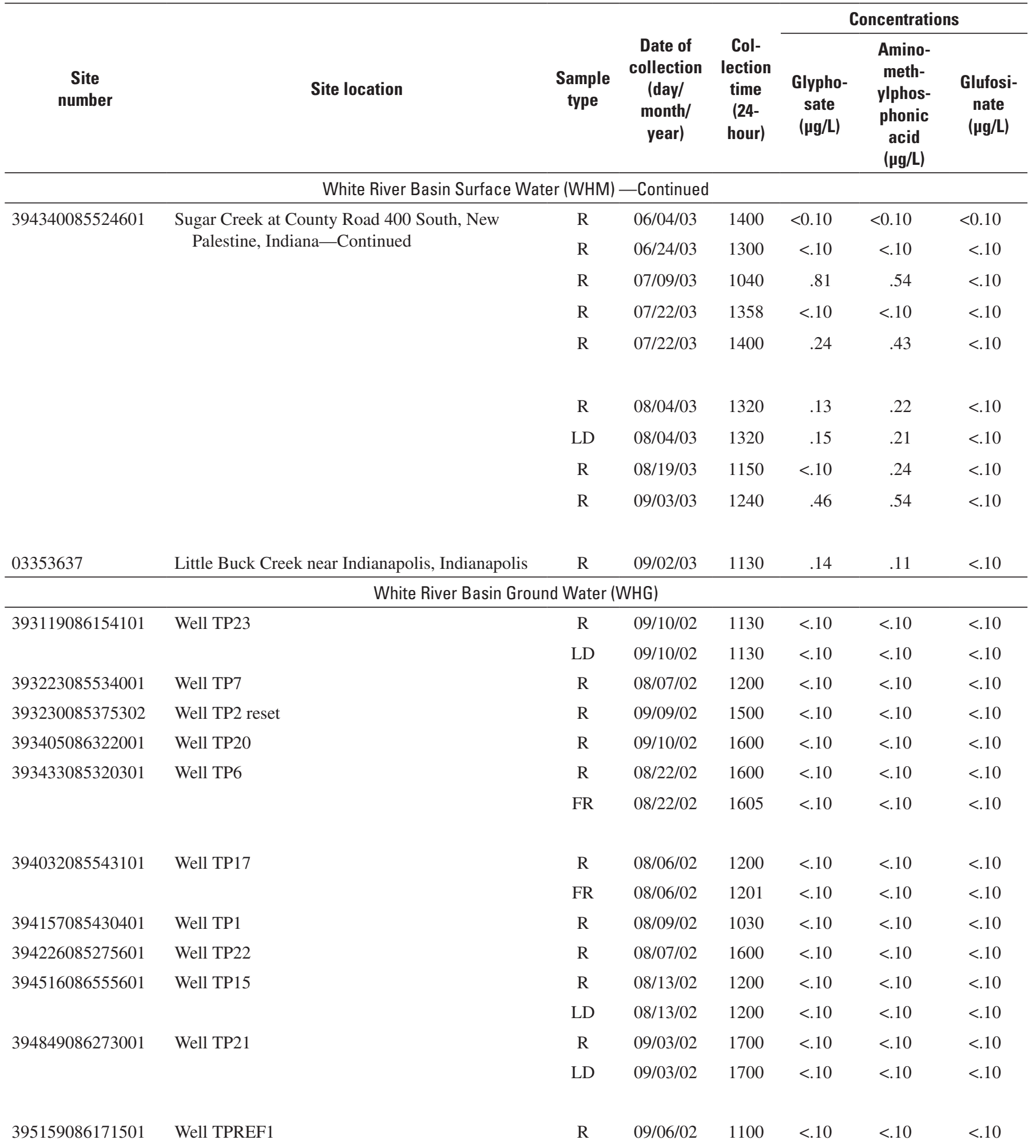


Table 5. Analytical results for glyphosate, aminomethylphosphonic acid, and glufosinate in National Water-Quality Assessment (NAWQA) Program surface- and ground-water studies, 2001-06.-Continued

[Location of NAWQA study units shown in figure 4. Sample type: FB, field blank; FR, field replicate; R, regular; LD, laboratory duplicate; --, no data; <, less than; $\mu \mathrm{g} / \mathrm{L}$, micrograms per liter]

\begin{tabular}{|c|c|c|c|c|c|c|c|}
\hline \multirow{2}{*}{$\begin{array}{c}\text { Site } \\
\text { number }\end{array}$} & \multirow[b]{2}{*}{ Site location } & \multirow[b]{2}{*}{$\begin{array}{c}\text { Sample } \\
\text { type }\end{array}$} & \multirow[b]{2}{*}{$\begin{array}{l}\text { Date of } \\
\text { collection } \\
\text { (day/ } \\
\text { month/ } \\
\text { year) }\end{array}$} & \multirow[b]{2}{*}{$\begin{array}{c}\text { Col- } \\
\text { lection } \\
\text { time } \\
\text { (24- } \\
\text { hour) }\end{array}$} & \multicolumn{3}{|c|}{ Concentrations } \\
\hline & & & & & $\begin{array}{c}\text { Glypho- } \\
\text { sate } \\
\text { (pg/L) }\end{array}$ & $\begin{array}{c}\text { Amino- } \\
\text { meth- } \\
\text { ylphos- } \\
\text { phonic } \\
\text { acid } \\
\text { ( } \mu \mathrm{g} / \mathrm{L})\end{array}$ & $\begin{array}{c}\text { Glufosi- } \\
\text { nate } \\
\text { ( } \mu \mathrm{g} / \mathrm{L})\end{array}$ \\
\hline \multirow[t]{2}{*}{395253086314001} & Well TP4 & $\mathrm{R}$ & 08/08/02 & 1305 & $<0.10$ & $<0.10$ & $<0.10$ \\
\hline & & $\mathrm{R}$ & 09/04/02 & 1030 & $<.10$ & $<.10$ & $<.10$ \\
\hline \multirow[t]{2}{*}{395633085460801} & Well TP5 & $\mathrm{R}$ & 08/19/02 & 1500 & $<.10$ & $<.10$ & $<.10$ \\
\hline & & FR & 08/19/02 & 1501 & $<.10$ & $<.10$ & $<.10$ \\
\hline 400356085562101 & Well TP18 & $\mathrm{R}$ & $08 / 15 / 02$ & 1300 & $<.10$ & $<.10$ & $<.10$ \\
\hline 400401085044701 & Well TP11 & $\mathrm{R}$ & 09/05/02 & 1630 & $<.10$ & $<.10$ & $<.10$ \\
\hline 400713086132801 & Well TP3 & $\mathrm{R}$ & 09/04/02 & 1500 & $<.10$ & $<.10$ & $<.10$ \\
\hline 400717085032801 & Well TP10 reset & $\mathrm{R}$ & 09/11/02 & 1130 & $<.10$ & $<.10$ & $<.10$ \\
\hline 400746084551101 & Well TP24 & $\mathrm{R}$ & 09/05/02 & 1300 & $<.10$ & $<.10$ & $<.10$ \\
\hline 400836085205901 & WellTP9 reset & $\mathrm{R}$ & 09/11/02 & 1600 & $<.10$ & $<.10$ & $<.10$ \\
\hline 400844086095201 & Well TP16 & $\mathrm{R}$ & 09/04/02 & 1800 & $<.10$ & $<.10$ & $<.10$ \\
\hline \multicolumn{8}{|c|}{ Willamette Basin Surface Water (WIL) } \\
\hline \multirow{9}{*}{14201300} & & LD & $10 / 16 / 02$ & 1220 & .65 & 3.4 & $<.10$ \\
\hline & & $\mathrm{R}$ & $10 / 16 / 02$ & 1228 & $<.10$ & .28 & $<.10$ \\
\hline & & $\mathrm{R}$ & $10 / 30 / 02$ & 1100 & .29 & 1.3 & $<.10$ \\
\hline & & $\mathrm{LD}$ & $10 / 30 / 02$ & 1100 & .74 & 1.2 & $<.10$ \\
\hline & & $\mathrm{R}$ & $11 / 06 / 02$ & 1110 & .25 & 1.1 & $<.10$ \\
\hline & & $\mathrm{R}$ & $11 / 20 / 02$ & 1130 & .21 & .90 & $<.10$ \\
\hline & & $\mathrm{R}$ & $12 / 04 / 02$ & 1200 & .14 & .95 & $<.10$ \\
\hline & & $\mathrm{R}$ & $12 / 19 / 02$ & 1020 & $<.10$ & .16 & $<.10$ \\
\hline & & $\mathrm{LD}$ & $12 / 19 / 02$ & 1020 & $<.10$ & .15 & $<.10$ \\
\hline
\end{tabular}


Table 5. Analytical results for glyphosate, aminomethylphosphonic acid, and glufosinate in National Water-Quality Assessment (NAWQA) Program surface- and ground-water studies, 2001-06.-Continued

[Location of NAWQA study units shown in figure 4. Sample type: FB, field blank; FR, field replicate; R, regular; LD, laboratory duplicate; --, no data; <, less than; $\mu \mathrm{g} / \mathrm{L}$, micrograms per liter]

\begin{tabular}{|c|c|c|c|c|c|c|c|}
\hline \multirow[b]{2}{*}{$\begin{array}{c}\text { Site } \\
\text { number }\end{array}$} & \multirow[b]{2}{*}{ Site location } & \multirow[b]{2}{*}{$\begin{array}{l}\text { Sample } \\
\text { type }\end{array}$} & \multirow[b]{2}{*}{$\begin{array}{c}\text { Date of } \\
\text { collection } \\
\text { (day/ } \\
\text { month/ } \\
\text { year) }\end{array}$} & \multirow[b]{2}{*}{$\begin{array}{c}\text { Col- } \\
\text { lection } \\
\text { time } \\
\text { (24- } \\
\text { hour) }\end{array}$} & \multicolumn{3}{|c|}{ Concentrations } \\
\hline & & & & & $\begin{array}{c}\text { Glypho- } \\
\text { sate } \\
\text { ( } \mathrm{gg} / \mathrm{L})\end{array}$ & $\begin{array}{c}\text { Amino- } \\
\text { meth- } \\
\text { ylphos- } \\
\text { phonic } \\
\text { acid } \\
\text { ( } \mu \mathrm{g} / \mathrm{L})\end{array}$ & $\begin{array}{c}\text { Glufosi- } \\
\text { nate } \\
\text { (رg/L) }\end{array}$ \\
\hline \multirow[t]{11}{*}{14201300} & \multirow{11}{*}{$\begin{array}{l}\text { Zollner Creek near Mount Angel, Oregon- } \\
\text { Continued }\end{array}$} & $\mathrm{R}$ & $01 / 09 / 03$ & 1050 & $<0.10$ & $<0.10$ & $<0.10$ \\
\hline & & $\mathrm{R}$ & 02/10/03 & 1140 & .17 & .10 & $<.10$ \\
\hline & & FR & $02 / 10 / 03$ & 1141 & .12 & .14 & $<.10$ \\
\hline & & $\mathrm{R}$ & 03/03/03 & 1240 & .78 & .45 & $<.10$ \\
\hline & & FB & $04 / 01 / 03$ & 1129 & $<.10$ & $<.10$ & $<.10$ \\
\hline & & $\mathrm{R}$ & $04 / 16 / 03$ & 1420 & .86 & .26 & $<.10$ \\
\hline & & $\mathrm{R}$ & $04 / 30 / 03$ & 1350 & .46 & .22 & $<.10$ \\
\hline & & $\mathrm{R}$ & $05 / 14 / 03$ & 1150 & .44 & .15 & $<.10$ \\
\hline & & $\mathrm{R}$ & 06/03/03 & 1120 & .21 & .52 & $<.10$ \\
\hline & & $\mathrm{R}$ & $06 / 25 / 03$ & 1140 & .16 & 1.7 & $<.10$ \\
\hline & & $\mathrm{R}$ & 07/09/03 & 1210 & $<.10$ & .66 & $<.10$ \\
\hline 441719123033101 & Well 64 & $\mathrm{R}$ & 07/10/02 & 1500 & $<.10$ & $<.10$ & $<.10$ \\
\hline 442208123080501 & Well 60 & $\mathrm{R}$ & 08/20/02 & 1700 & $<.10$ & $<.10$ & $<.10$ \\
\hline 442252122592201 & Well 61 & $\mathrm{R}$ & 07/10/02 & 1700 & $<.10$ & $<.10$ & $<.10$ \\
\hline 442642123091201 & Well 58 & $\mathrm{R}$ & 08/20/02 & 1400 & $<.10$ & $<.10$ & $<.10$ \\
\hline 443226123121701 & Well 54 & $\mathrm{R}$ & 07/08/02 & 1600 & $<.10$ & $<.10$ & $<.10$ \\
\hline \multirow[t]{3}{*}{443437123133901} & Well 49 & $\mathrm{R}$ & 07/08/02 & 1300 & $<.10$ & $<.10$ & $<.10$ \\
\hline & & FR & 07/08/02 & 1301 & $<.10$ & $<.10$ & $<.10$ \\
\hline & & LD & 07/08/02 & 1301 & $<.10$ & $<.10$ & $<.10$ \\
\hline \multirow[t]{2}{*}{443646122552701} & Well 51 & $\mathrm{R}$ & $06 / 28 / 02$ & 1300 & $<.10$ & $<.10$ & $<.10$ \\
\hline & & FR & $06 / 28 / 02$ & 1308 & $<.10$ & $<.10$ & $<.10$ \\
\hline 444055123035401 & Well 46 & $\mathrm{R}$ & $07 / 11 / 02$ & 1700 & $<.10$ & $<.10$ & $<.10$ \\
\hline
\end{tabular}


Table 5. Analytical results for glyphosate, aminomethylphosphonic acid, and glufosinate in National Water-Quality Assessment (NAWQA) Program surface- and ground-water studies, 2001-06.-Continued

[Location of NAWQA study units shown in figure 4. Sample type: FB, field blank; FR, field replicate; R, regular; LD, laboratory duplicate; --, no data; <, less than; $\mu \mathrm{g} / \mathrm{L}$, micrograms per liter]

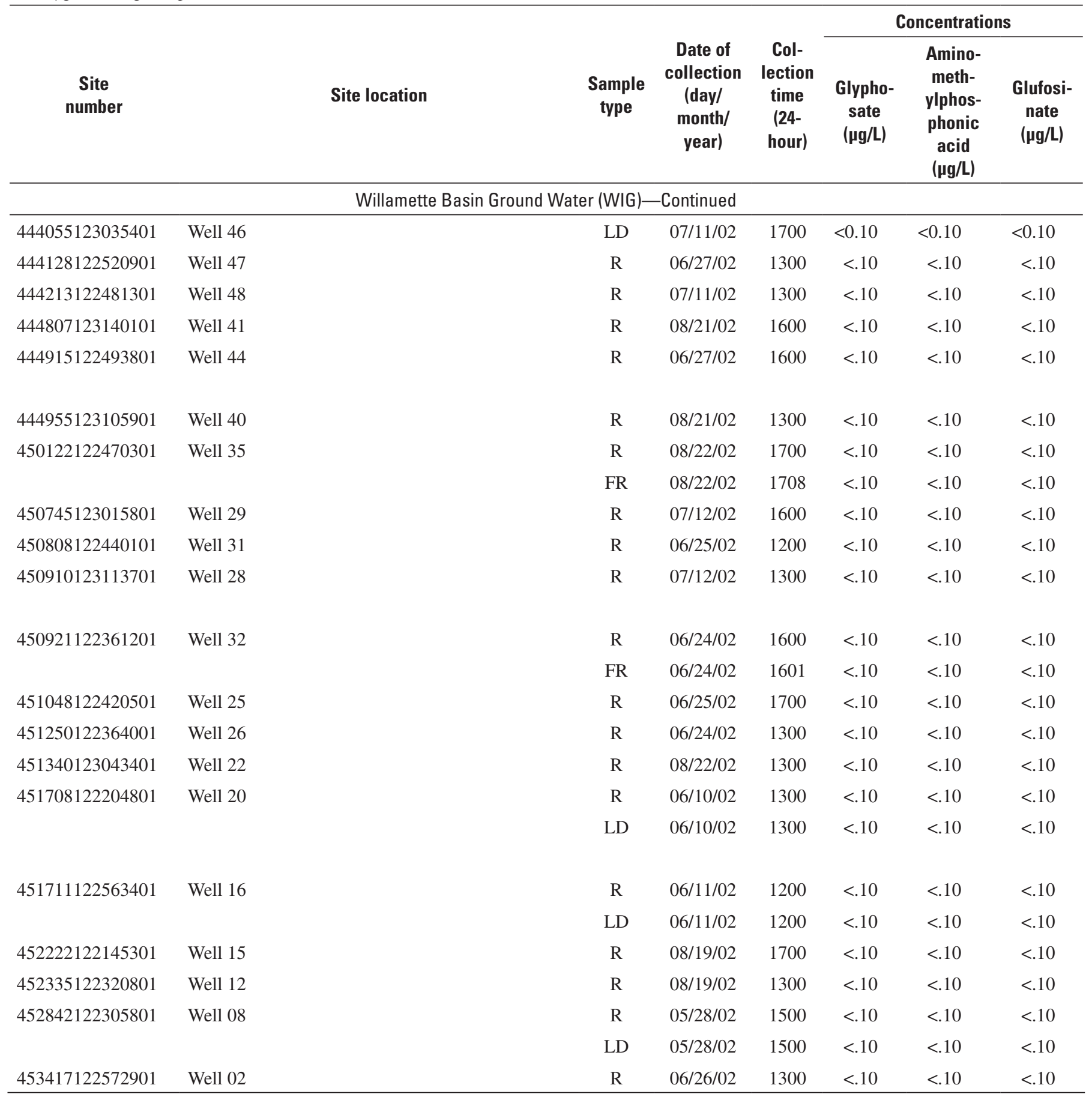


Table 6. Analytical results for glyphosate, aminomethylphosphonic acid, and glufosinate in Toxic Substances Hydrology Program studies, 2001-06. [Sample type: FB, field blank; FR, field replicate; R, regular; LD, laboratory duplicate; --, no data; <, less than; $\mu \mathrm{g} / \mathrm{L}$, micrograms per liter]

\begin{tabular}{|c|c|c|c|c|c|c|c|}
\hline \multirow[b]{2}{*}{ Site number } & \multirow[b]{2}{*}{ Site location } & \multirow[b]{2}{*}{$\begin{array}{l}\text { Sam- } \\
\text { ple } \\
\text { type }\end{array}$} & \multirow[b]{2}{*}{$\begin{array}{c}\text { Date of } \\
\text { collection } \\
\text { (month/day/ } \\
\text { year) }\end{array}$} & \multirow[b]{2}{*}{$\begin{array}{l}\text { Col- } \\
\text { lec- } \\
\text { tion } \\
\text { time } \\
\text { (24- } \\
\text { hour) }\end{array}$} & \multicolumn{3}{|c|}{ Concentrations } \\
\hline & & & & & $\begin{array}{c}\text { Glypho- } \\
\text { sate } \\
\text { ( } \mu \mathrm{g} / \mathrm{L})\end{array}$ & $\begin{array}{l}\text { Amino- } \\
\text { meth- } \\
\text { ylphos- } \\
\text { phonic } \\
\text { acid } \\
\text { ( } \mu \mathrm{g} / \mathrm{L})\end{array}$ & $\begin{array}{c}\text { Glufos- } \\
\text { inate } \\
\text { ( } \mu \mathrm{g} / \mathrm{L} \text { ) }\end{array}$ \\
\hline \multicolumn{8}{|c|}{ Reconnaissance data for glyphosate in nine Midwestern States } \\
\hline \multicolumn{8}{|c|}{ Illinois } \\
\hline \multirow[t]{4}{*}{03378000} & Bonpas Creek at Browns & $\mathrm{R}$ & 06/06/02 & 1030 & $<0.10$ & 0.33 & $<0.10$ \\
\hline & & FR & 06/06/02 & 1031 & $<.10$ & .26 & $<.10$ \\
\hline & & $\mathrm{R}$ & $06 / 25 / 02$ & 1300 & 4.5 & .91 & $<.10$ \\
\hline & & $\mathrm{R}$ & $09 / 25 / 02$ & 1015 & .44 & .55 & $<.10$ \\
\hline \multirow[t]{4}{*}{03381495} & Little Wabash River at Main Street at Carmi & $\mathrm{R}$ & 06/06/02 & 1230 & $<.10$ & $<.10$ & $<.10$ \\
\hline & & $\mathrm{R}$ & $06 / 25 / 02$ & 1430 & 1.4 & .83 & $<.10$ \\
\hline & & FR & $06 / 25 / 02$ & 1431 & 2.3 & .91 & $<.10$ \\
\hline & & $\mathrm{R}$ & $09 / 25 / 02$ & 0830 & .34 & .80 & $<.10$ \\
\hline \multirow[t]{3}{*}{05439500} & South Branch Kishwaukee River near Fairdale & $\mathrm{R}$ & $05 / 14 / 02$ & 1600 & .33 & .19 & $<.10$ \\
\hline & & $\mathrm{R}$ & 08/02/02 & 1430 & $<.10$ & .26 & $<.10$ \\
\hline & & $\mathrm{R}$ & $09 / 25 / 02$ & 0930 & $<.10$ & .28 & $<.10$ \\
\hline \multirow[t]{4}{*}{05526000} & Iroquois River near Chebanse & $\mathrm{R}$ & 06/06/02 & 0925 & $<.10$ & $<.10$ & $<.10$ \\
\hline & & $\mathrm{R}$ & $06 / 28 / 02$ & 0910 & .19 & .29 & $<.10$ \\
\hline & & $\mathrm{LD}$ & $06 / 28 / 02$ & 0910 & .25 & .18 & $<.10$ \\
\hline & & $\mathrm{R}$ & $09 / 25 / 02$ & 1345 & $<.10$ & .21 & $<.10$ \\
\hline \multirow[t]{3}{*}{05540500} & Dupage River at Shorewood & $\mathrm{R}$ & $05 / 15 / 02$ & 1030 & $<.10$ & .10 & $<.10$ \\
\hline & & $\mathrm{R}$ & $06 / 27 / 02$ & 1240 & .34 & .44 & $<.10$ \\
\hline & & $\mathrm{R}$ & $09 / 25 / 02$ & 1145 & $<.10$ & .89 & $<.10$ \\
\hline \multirow[t]{3}{*}{05569500} & Spoon River at London Mills & $\mathrm{R}$ & 05/14/02 & 1035 & .19 & .23 & $<.10$ \\
\hline & & $\mathrm{R}$ & 07/30/02 & 1000 & $<.10$ & .24 & $<.10$ \\
\hline & & $\mathrm{R}$ & $09 / 24 / 02$ & 0905 & $<.10$ & .10 & $<.10$ \\
\hline \multirow[t]{5}{*}{05576500} & Sangamon River at Riverton & $\mathrm{R}$ & $05 / 29 / 02$ & 1015 & .36 & .16 & $<.10$ \\
\hline & & $\mathrm{R}$ & 07/17/02 & 1515 & $<.10$ & .61 & $<.10$ \\
\hline & & LD & 07/17/02 & 1515 & $<.10$ & .74 & $<.10$ \\
\hline & & $\mathrm{R}$ & $09 / 25 / 02$ & 0845 & $<.10$ & 1.8 & $<.10$ \\
\hline & & LD & $09 / 25 / 02$ & 0845 & $<.10$ & 1.7 & $<.10$ \\
\hline 05587000 & Macoupin Creek near Kane & $\mathrm{R}$ & 06/06/02 & 1000 & $<.10$ & $<.10$ & $<.10$ \\
\hline
\end{tabular}


Table 6. Analytical results for glyphosate, aminomethylphosphonic acid, and glufosinate in Toxic Substances Hydrology Program studies, 2001-06. Continued

[Sample type: FB, field blank; FR, field replicate; R, regular; LD, laboratory duplicate; --, no data; <, less than; $\mu \mathrm{g} / \mathrm{L}$, micrograms per liter]

\begin{tabular}{|c|c|c|c|c|c|c|c|}
\hline \multirow[b]{2}{*}{ Site number } & \multirow[b]{2}{*}{ Site location } & \multirow[b]{2}{*}{$\begin{array}{l}\text { Sam- } \\
\text { ple } \\
\text { type }\end{array}$} & \multirow[b]{2}{*}{$\begin{array}{c}\text { Date of } \\
\text { collection } \\
\text { (month/day/ } \\
\text { year) }\end{array}$} & \multirow[b]{2}{*}{$\begin{array}{l}\text { Col- } \\
\text { lec- } \\
\text { tion } \\
\text { time } \\
(24- \\
\text { hour) }\end{array}$} & \multicolumn{3}{|c|}{ Concentrations } \\
\hline & & & & & $\begin{array}{l}\text { Glypho- } \\
\text { sate } \\
\text { ( } \mu \mathrm{g} / \mathrm{L})\end{array}$ & $\begin{array}{l}\text { Amino- } \\
\text { meth- } \\
\text { ylphos- } \\
\text { phonic } \\
\text { acid } \\
\text { ( } \mathrm{\mu g} / \mathrm{L})\end{array}$ & $\begin{array}{l}\text { Glufos- } \\
\text { inate } \\
(\mu \mathrm{g} / \mathrm{L})\end{array}$ \\
\hline \multicolumn{8}{|c|}{ Reconnaissance data for glyphosate in nine Midwestern States-Continued } \\
\hline \multicolumn{8}{|c|}{ Illinois-Continued } \\
\hline \multirow[t]{2}{*}{05587000} & Macoupin Creek near Kane-Continued & $\mathrm{R}$ & 07/29/02 & 1115 & 0.13 & 0.39 & $<0.10$ \\
\hline & & $\mathrm{R}$ & 09/24/02 & 1120 & $<.10$ & .51 & $<.10$ \\
\hline \multirow[t]{3}{*}{05592100} & Kaskaskia River near Cowden & $\mathrm{R}$ & 05/29/02 & 0930 & .20 & .17 & $<.10$ \\
\hline & & $\mathrm{R}$ & $06 / 25 / 02$ & 1200 & $<.10$ & .27 & $<.10$ \\
\hline & & $\mathrm{R}$ & 09/25/02 & 1330 & $<.10$ & .21 & $<.10$ \\
\hline \multirow[t]{3}{*}{05594000} & Shoal Creek near Breese & $\mathrm{R}$ & 06/06/02 & 1330 & $<.10$ & .22 & $<.10$ \\
\hline & & $\mathrm{R}$ & 07/29/02 & 1410 & .17 & .22 & $<.10$ \\
\hline & & $\mathrm{R}$ & 09/24/02 & 1420 & .14 & .23 & $<.10$ \\
\hline \multicolumn{8}{|c|}{ Indiana } \\
\hline \multirow[t]{4}{*}{03275000} & Whitewater River near Alpine & FB & 06/14/02 & 1145 & $<.10$ & $<.10$ & $<.10$ \\
\hline & & $\mathrm{R}$ & 06/14/02 & 1230 & 1.0 & .43 & $<.10$ \\
\hline & & $\mathrm{R}$ & 06/28/02 & 1205 & .34 & .28 & $<.10$ \\
\hline & & $\mathrm{R}$ & $10 / 29 / 02$ & 1715 & $<.10$ & .17 & $<.10$ \\
\hline \multirow[t]{3}{*}{03302800} & Blue River at Fredericksburg & $\mathrm{R}$ & 06/06/02 & 1130 & .34 & .28 & $<.10$ \\
\hline & & $\mathrm{R}$ & 08/21/02 & 1130 & $<.10$ & $<.10$ & $<.10$ \\
\hline & & $\mathrm{R}$ & 09/27/02 & 1200 & .26 & .36 & $<.10$ \\
\hline \multirow[t]{4}{*}{03328500} & Eel River near Logansport & $\mathrm{R}$ & 06/05/02 & 1200 & $<.10$ & $<.10$ & $<.10$ \\
\hline & & $\mathrm{R}$ & 07/30/02 & 1130 & $<.10$ & .18 & $<.10$ \\
\hline & & $\mathrm{R}$ & 07/31/02 & 1250 & $<.10$ & .31 & $<.10$ \\
\hline & & $\mathrm{R}$ & $12 / 10 / 02$ & 1000 & $<.10$ & $<.10$ & $<.10$ \\
\hline \multirow[t]{4}{*}{03333450} & Wildcat Creek near Jerome & $\mathrm{R}$ & 05/30/02 & 1250 & .16 & .12 & $<.10$ \\
\hline & & $\mathrm{R}$ & 08/19/02 & 1110 & $<.10$ & .13 & $<.10$ \\
\hline & & $\mathrm{R}$ & 09/20/02 & 1316 & $<.10$ & .10 & $<.10$ \\
\hline & & $\mathrm{LD}$ & $09 / 20 / 02$ & 1316 & $<.10$ & .10 & $<.10$ \\
\hline \multirow[t]{3}{*}{03335000} & Wildcat Creek near Lafayette & $\mathrm{R}$ & 05/30/02 & 1630 & .19 & .23 & $<.10$ \\
\hline & & $\mathrm{R}$ & 07/23/02 & 1000 & $<.10$ & .36 & $<.10$ \\
\hline & & $\mathrm{R}$ & $11 / 12 / 02$ & 1215 & $<.10$ & .79 & $<.10$ \\
\hline
\end{tabular}


Table 6. Analytical results for glyphosate, aminomethylphosphonic acid, and glufosinate in Toxic Substances Hydrology Program studies, 2001-06.— Continued

[Sample type: FB, field blank; FR, field replicate; R, regular; LD, laboratory duplicate; --, no data; <, less than; $\mu \mathrm{g} / \mathrm{L}$, micrograms per liter]

\begin{tabular}{|c|c|c|c|c|c|c|c|}
\hline \multirow[b]{2}{*}{ Site number } & \multirow[b]{2}{*}{ Site location } & \multirow[b]{2}{*}{$\begin{array}{l}\text { Sam- } \\
\text { ple } \\
\text { type }\end{array}$} & \multirow[b]{2}{*}{$\begin{array}{c}\text { Date of } \\
\text { collection } \\
\text { (month/day/ } \\
\text { year) }\end{array}$} & \multirow[b]{2}{*}{$\begin{array}{l}\text { Col- } \\
\text { lec- } \\
\text { tion } \\
\text { time } \\
\text { (24- } \\
\text { hour) }\end{array}$} & \multicolumn{3}{|c|}{ Concentrations } \\
\hline & & & & & 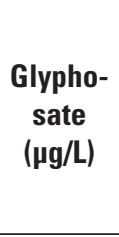 & $\begin{array}{c}\text { Amino- } \\
\text { meth- } \\
\text { ylphos- } \\
\text { phonic } \\
\text { acid } \\
\text { ( } \mathrm{rg} / \mathrm{L} \text { ) }\end{array}$ & $\begin{array}{c}\text { Glufos- } \\
\text { inate } \\
\text { ( } \mu \mathrm{g} / \mathrm{L} \text { ) }\end{array}$ \\
\hline \multicolumn{8}{|c|}{ Reconnaissance data for glyphosate in nine Midwestern States-Continued } \\
\hline \multicolumn{8}{|c|}{ Indiana-Continued } \\
\hline \multirow[t]{5}{*}{03362500} & Sugar Creek near Edinburgh & $\mathrm{R}$ & $06 / 07 / 02$ & 1345 & 0.45 & 0.34 & $<0.10$ \\
\hline & & $\mathrm{R}$ & $06 / 26 / 02$ & 1020 & 1.5 & .38 & $<.10$ \\
\hline & & $\mathrm{R}$ & $06 / 26 / 02$ & 1120 & 1.0 & .51 & $<.10$ \\
\hline & & FR & $06 / 26 / 02$ & 1121 & .58 & .33 & $<.10$ \\
\hline & & $\mathrm{R}$ & $10 / 26 / 02$ & 1340 & .30 & .62 & $<.10$ \\
\hline \multirow[t]{5}{*}{03371500} & East Fork White River near Bedford & $\mathrm{R}$ & $06 / 06 / 02$ & 1600 & .23 & .22 & $<.10$ \\
\hline & & $\mathrm{R}$ & $07 / 23 / 02$ & 1605 & $<.10$ & .37 & $<.10$ \\
\hline & & $\mathrm{LD}$ & $07 / 23 / 02$ & 1605 & $<.10$ & .42 & $<.10$ \\
\hline & & $\mathrm{R}$ & $10 / 01 / 02$ & 1140 & .13 & .34 & $<.10$ \\
\hline & & FR & $10 / 01 / 02$ & 1141 & .20 & .33 & $<.10$ \\
\hline \multicolumn{8}{|c|}{ lowa } \\
\hline \multirow[t]{4}{*}{05411850} & Turkey River near Eldorado & $\mathrm{R}$ & $05 / 14 / 02$ & 1400 & $<.10$ & $<.10$ & $<.10$ \\
\hline & & FR & $05 / 14 / 02$ & 1405 & $<.10$ & $<.10$ & $<.10$ \\
\hline & & $\mathrm{R}$ & $06 / 24 / 02$ & 0950 & $<.10$ & $<.10$ & $<.10$ \\
\hline & & $\mathrm{R}$ & $10 / 29 / 02$ & 1300 & $<.10$ & $<.10$ & $<.10$ \\
\hline \multirow[t]{3}{*}{05421000} & Wapsipinicon River at Independence & $\mathrm{R}$ & $05 / 14 / 02$ & 1100 & $<.10$ & $<.10$ & $<.10$ \\
\hline & & $\mathrm{R}$ & $06 / 24 / 02$ & 1245 & $<.10$ & $<.10$ & $<.10$ \\
\hline & & $\mathrm{R}$ & $10 / 29 / 2002$ & 0930 & $<.10$ & $<.10$ & $<.10$ \\
\hline \multirow[t]{3}{*}{05455100} & Old Mans Creek near Iowa City & $\mathrm{R}$ & $05 / 13 / 02$ & 1100 & $<.10$ & $<.10$ & $<.10$ \\
\hline & & $\mathrm{R}$ & $06 / 27 / 02$ & 0955 & .46 & .26 & $<.10$ \\
\hline & & $\mathrm{R}$ & $10 / 30 / 02$ & 1140 & $<.10$ & .13 & $<.10$ \\
\hline \multirow[t]{3}{*}{05472500} & North Skunk River near Sigourney & $\mathrm{R}$ & $05 / 13 / 02$ & 1630 & $<.10$ & $<.10$ & $<.10$ \\
\hline & & $\mathrm{R}$ & $07 / 08 / 02$ & 1015 & $<.10$ & .18 & $<.10$ \\
\hline & & $\mathrm{R}$ & $10 / 29 / 2002$ & 1435 & $<.10$ & $<.10$ & $<.10$ \\
\hline \multirow[t]{3}{*}{05474000} & Skunk River at Augusta & $\mathrm{R}$ & $05 / 13 / 02$ & 1230 & $<.10$ & $<.10$ & $<.10$ \\
\hline & & $\mathrm{R}$ & $06 / 27 / 02$ & 1235 & .36 & .26 & $<.10$ \\
\hline & & $\mathrm{R}$ & $10 / 29 / 2002$ & 1015 & $<.10$ & .11 & $<.10$ \\
\hline
\end{tabular}


Table 6. Analytical results for glyphosate, aminomethylphosphonic acid, and glufosinate in Toxic Substances Hydrology Program studies, 2001-06.Continued

[Sample type: FB, field blank; FR, field replicate; R, regular; LD, laboratory duplicate; --, no data; <, less than; $\mu \mathrm{g} / \mathrm{L}$, micrograms per liter]

\begin{tabular}{|c|c|c|c|c|c|c|c|}
\hline \multirow[b]{2}{*}{ Site number } & \multirow[b]{2}{*}{ Site location } & \multirow[b]{2}{*}{$\begin{array}{l}\text { Sam- } \\
\text { ple } \\
\text { type }\end{array}$} & \multirow[b]{2}{*}{$\begin{array}{c}\text { Date of } \\
\text { collection } \\
\text { (month/day/ } \\
\text { year) }\end{array}$} & \multirow[b]{2}{*}{$\begin{array}{l}\text { Col- } \\
\text { lec- } \\
\text { tion } \\
\text { time } \\
(24- \\
\text { hour) }\end{array}$} & \multicolumn{3}{|c|}{ Concentrations } \\
\hline & & & & & $\begin{array}{c}\text { Glypho- } \\
\text { sate } \\
\text { ( } \mu \mathrm{g} / \mathrm{L} \text { ) }\end{array}$ & $\begin{array}{l}\text { Amino- } \\
\text { meth- } \\
\text { ylphos- } \\
\text { phonic } \\
\text { acid } \\
\text { ( } \mathrm{\mu g} / \mathrm{L})\end{array}$ & $\begin{array}{c}\text { Glufos- } \\
\text { inate } \\
(\mu \mathrm{g} / \mathrm{L})\end{array}$ \\
\hline \multicolumn{8}{|c|}{ Reconnaissance data for glyphosate in nine Midwestern States-Continued } \\
\hline \multicolumn{8}{|c|}{ lowa-Continued } \\
\hline \multirow[t]{3}{*}{05480500} & Des Moines River at Fort Dodge & $\mathrm{R}$ & 05/17/02 & 1230 & $<0.10$ & $<0.10$ & $<0.10$ \\
\hline & & $\mathrm{R}$ & 06/13/02 & 0730 & $<.10$ & .19 & $<.10$ \\
\hline & & $\mathrm{R}$ & $10 / 30 / 02$ & 1500 & $<.10$ & .10 & $<.10$ \\
\hline \multirow[t]{4}{*}{05484500} & Racoon River at Van Meter & $\mathrm{R}$ & 05/17/02 & 0930 & $<.10$ & $<.10$ & $<.10$ \\
\hline & & $\mathrm{R}$ & $06 / 12 / 02$ & 1630 & $<.10$ & .17 & $<.10$ \\
\hline & & FR & $06 / 12 / 02$ & 1635 & .27 & .25 & $<.10$ \\
\hline & & $\mathrm{R}$ & $10 / 30 / 02$ & 1200 & $<.10$ & $<.10$ & $<.10$ \\
\hline \multirow[t]{3}{*}{06606600} & Little Sioux River at Correctionville & $\mathrm{R}$ & 05/17/02 & 1615 & $<.10$ & $<.10$ & $<.10$ \\
\hline & & $\mathrm{R}$ & 06/11/02 & 1100 & $<.10$ & .27 & $<.10$ \\
\hline & & $\mathrm{R}$ & $10 / 31 / 02$ & 0930 & $<.10$ & .13 & $<.10$ \\
\hline \multirow[t]{4}{*}{06607200} & Maple River at Mapleton & $\mathrm{R}$ & $05 / 29 / 02$ & 1200 & $<.10$ & $<.10$ & $<.10$ \\
\hline & & LD & $05 / 29 / 02$ & 1200 & $<.10$ & $<.10$ & $<.10$ \\
\hline & & $\mathrm{R}$ & 06/11/02 & 1330 & .36 & .64 & .14 \\
\hline & & $\mathrm{R}$ & $10 / 31 / 02$ & 1200 & $<.10$ & .42 & $<.10$ \\
\hline \multirow[t]{3}{*}{06609500} & Boyer River at Logan & $\mathrm{R}$ & $05 / 29 / 02$ & 0945 & $<.10$ & .33 & $<.10$ \\
\hline & & $\mathrm{R}$ & 06/11/02 & 1545 & .32 & .45 & $<.10$ \\
\hline & & $\mathrm{R}$ & $11 / 01 / 02$ & 0900 & .12 & .20 & $<.10$ \\
\hline \multicolumn{8}{|c|}{ Kansas } \\
\hline \multirow[t]{5}{*}{06885500} & Black Vermillion near Frankfort & $\mathrm{R}$ & 05/07/02 & 1145 & $<.10$ & $<.10$ & $<.10$ \\
\hline & & LD & 05/07/02 & 1145 & $<.10$ & $<.10$ & $<.10$ \\
\hline & & $\mathrm{R}$ & 05/07/02 & 1150 & $<.10$ & $<.10$ & $<.10$ \\
\hline & & $\mathrm{R}$ & 08/17/02 & 1235 & $<.10$ & .11 & $<.10$ \\
\hline & & $\mathrm{R}$ & $10 / 03 / 02$ & 1135 & 8.7 & 3.6 & $<.10$ \\
\hline \multirow[t]{4}{*}{06890100} & Delaware River near Muscotah & $\mathrm{R}$ & 05/07/02 & 1315 & $<.10$ & $<.10$ & $<.10$ \\
\hline & & $\mathrm{R}$ & $06 / 27 / 02$ & 0950 & 1.7 & .41 & .26 \\
\hline & & LD & $06 / 27 / 02$ & 0950 & 1.6 & .63 & .27 \\
\hline & & $\mathrm{R}$ & $09 / 19 / 02$ & 1110 & $<.10$ & $<.10$ & $<.10$ \\
\hline
\end{tabular}


Table 6. Analytical results for glyphosate, aminomethylphosphonic acid, and glufosinate in Toxic Substances Hydrology Program studies, 2001-06.— Continued

[Sample type: FB, field blank; FR, field replicate; R, regular; LD, laboratory duplicate; --, no data; <, less than; $\mu \mathrm{g} / \mathrm{L}$, micrograms per liter]

\begin{tabular}{|c|c|c|c|c|c|c|c|}
\hline \multirow[b]{2}{*}{ Site number } & \multirow[b]{2}{*}{ Site location } & \multirow[b]{2}{*}{$\begin{array}{l}\text { Sam- } \\
\text { ple } \\
\text { type }\end{array}$} & \multirow[b]{2}{*}{$\begin{array}{c}\text { Date of } \\
\text { collection } \\
\text { (month/day/ } \\
\text { year) }\end{array}$} & \multirow[b]{2}{*}{$\begin{array}{l}\text { Col- } \\
\text { lec- } \\
\text { tion } \\
\text { time } \\
\text { (24- } \\
\text { hour) }\end{array}$} & \multicolumn{3}{|c|}{ Concentrations } \\
\hline & & & & & $\begin{array}{l}\text { Glypho- } \\
\text { sate } \\
\text { ( } \mu \mathrm{g} / \mathrm{L})\end{array}$ & 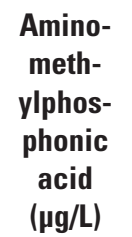 & $\begin{array}{l}\text { Glufos- } \\
\text { inate } \\
(\mu \mathrm{g} / \mathrm{L})\end{array}$ \\
\hline \multicolumn{8}{|c|}{ Reconnaissance data for glyphosate in nine Midwestern States-Continued } \\
\hline \multicolumn{8}{|c|}{ Minnesota } \\
\hline \multirow[t]{3}{*}{05317000} & Cottonwood River near New Ulm & $\mathrm{R}$ & $05 / 30 / 02$ & 1350 & $<0.10$ & $<0.10$ & $<0.10$ \\
\hline & & $\mathrm{R}$ & $06 / 25 / 02$ & 1620 & .77 & .42 & $<.10$ \\
\hline & & $\mathrm{R}$ & $10 / 04 / 02$ & 0935 & $<.10$ & $<.10$ & $<.10$ \\
\hline \multirow[t]{3}{*}{05320270} & Little Cobb River near Beauford & $\mathrm{R}$ & 06/06/02 & 1315 & $<.10$ & $<.10$ & $<.10$ \\
\hline & & $\mathrm{R}$ & 06/27/02 & 1045 & .30 & .15 & $<.10$ \\
\hline & & $\mathrm{R}$ & $10 / 02 / 02$ & 1105 & $<.10$ & $<.10$ & $<.10$ \\
\hline \multirow[t]{4}{*}{05476000} & Des Moines River at Jackson & $\mathrm{R}$ & $05 / 31 / 02$ & 0720 & $<.10$ & $<.10$ & $<.10$ \\
\hline & & $\mathrm{R}$ & $06 / 25 / 02$ & 1215 & $<.10$ & $<.10$ & $<.10$ \\
\hline & & FR & $06 / 25 / 02$ & 1220 & $<.10$ & $<.10$ & $<.10$ \\
\hline & & $\mathrm{R}$ & $10 / 08 / 02$ & 1145 & $<.10$ & $<.10$ & $<.10$ \\
\hline \multicolumn{8}{|c|}{ Missouri } \\
\hline \multirow[t]{4}{*}{06817700} & Nodaway River near Graham & $\mathrm{R}$ & $05 / 14 / 02$ & 1230 & $<.10$ & $<.10$ & $<.10$ \\
\hline & & LD & $05 / 14 / 02$ & 1230 & $<.10$ & $<.10$ & $<.10$ \\
\hline & & $\mathrm{R}$ & $07 / 29 / 02$ & 1200 & $<.10$ & .36 & $<.10$ \\
\hline & & $\mathrm{R}$ & $10 / 04 / 02$ & 1215 & $<.10$ & .25 & $<.10$ \\
\hline \multicolumn{8}{|c|}{ Nebraska } \\
\hline \multirow[t]{3}{*}{06804000} & Wahoo Creek at Ithaca & $\mathrm{R}$ & 05/13/02 & 1330 & $<.10$ & .19 & $<.10$ \\
\hline & & $\mathrm{R}$ & $06 / 12 / 02$ & 0840 & $<.10$ & .38 & $<.10$ \\
\hline & & $\mathrm{R}$ & $10 / 04 / 02$ & 0930 & $<.10$ & .65 & $<.10$ \\
\hline \multirow[t]{4}{*}{06815000} & Big Nemaha River at Falls City & $\mathrm{R}$ & 05/06/02 & 1400 & $<.10$ & $<.10$ & $<.10$ \\
\hline & & $\mathrm{LD}$ & 05/06/02 & 1400 & $<.10$ & $<.10$ & $<.10$ \\
\hline & & $\mathrm{R}$ & $07 / 12 / 02$ & 1400 & $<.10$ & .19 & $<.10$ \\
\hline & & $\mathrm{R}$ & $10 / 04 / 02$ & 1520 & $<.10$ & .34 & $<.10$ \\
\hline \multirow[t]{3}{*}{06880800} & West Fork Big Blue River near Dorchester & $\mathrm{R}$ & 05/07/02 & 0900 & .11 & 1.3 & $<.10$ \\
\hline & & $\mathrm{R}$ & $07 / 26 / 02$ & 1100 & .17 & 2.0 & $<.10$ \\
\hline & & $\mathrm{R}$ & $10 / 04 / 02$ & 0920 & .21 & 1.3 & $<.10$ \\
\hline \multirow[t]{2}{*}{06882000} & Big Blue River at Barneston & $\mathrm{R}$ & 05/07/02 & 1530 & .31 & 1.3 & $<.10$ \\
\hline & & $\mathrm{R}$ & 06/15/02 & 0900 & .21 & 1.8 & $<.10$ \\
\hline
\end{tabular}


Table 6. Analytical results for glyphosate, aminomethylphosphonic acid, and glufosinate in Toxic Substances Hydrology Program studies, 2001-06. Continued

[Sample type: FB, field blank; FR, field replicate; R, regular; LD, laboratory duplicate; --, no data; <, less than; $\mu \mathrm{g} / \mathrm{L}$, micrograms per liter]

\begin{tabular}{|c|c|c|c|c|c|c|c|}
\hline \multirow[b]{2}{*}{ Site number } & \multirow[b]{2}{*}{ Site location } & \multirow[b]{2}{*}{$\begin{array}{l}\text { Sam- } \\
\text { ple } \\
\text { type }\end{array}$} & \multirow[b]{2}{*}{$\begin{array}{c}\text { Date of } \\
\text { collection } \\
\text { (month/day/ } \\
\text { year) }\end{array}$} & \multirow[b]{2}{*}{$\begin{array}{l}\text { Col- } \\
\text { lec- } \\
\text { tion } \\
\text { time } \\
\text { (24- } \\
\text { hour) }\end{array}$} & \multicolumn{3}{|c|}{ Concentrations } \\
\hline & & & & & $\begin{array}{c}\text { Glypho- } \\
\text { sate } \\
\text { ( } \mu \mathrm{g} / \mathrm{L})\end{array}$ & $\begin{array}{c}\text { Amino- } \\
\text { meth- } \\
\text { ylphos- } \\
\text { phonic } \\
\text { acid } \\
\text { ( } \mathrm{gg} / \mathrm{L} \text { ) }\end{array}$ & $\begin{array}{c}\text { Glufos- } \\
\text { inate } \\
(\mu \mathrm{g} / \mathrm{L})\end{array}$ \\
\hline \multicolumn{8}{|c|}{ Reconnaissance data for glyphosate in nine Midwestern States-Continued } \\
\hline \multicolumn{8}{|c|}{ Nebraska-Continued } \\
\hline \multirow[t]{3}{*}{06882000} & Big Blue River at Barneston-Continued & $\mathrm{R}$ & $10 / 04 / 02$ & 1300 & 0.54 & 1.3 & $<0.10$ \\
\hline & & $\mathrm{R}$ & $10 / 04 / 02$ & 1310 & .36 & 1.2 & $<.10$ \\
\hline & & $\mathrm{R}$ & $10 / 04 / 02$ & 1310 & .46 & 1.5 & $<.10$ \\
\hline \multirow[t]{3}{*}{06884000} & Little Blue River near Fairbury & $\mathrm{R}$ & 05/07/02 & 1230 & $<.10$ & .42 & $<.10$ \\
\hline & & $\mathrm{R}$ & 06/13/02 & 0930 & .52 & .64 & $<.10$ \\
\hline & & $\mathrm{R}$ & $10 / 02 / 02$ & 2000 & .33 & .71 & $<.10$ \\
\hline \multicolumn{8}{|c|}{ Ohio } \\
\hline \multirow[t]{3}{*}{03157000} & Clear Creek near Rockbridge & $\mathrm{R}$ & 06/05/02 & 1115 & .27 & .23 & $<.10$ \\
\hline & & $\mathrm{R}$ & 07/29/02 & 1200 & $<.10$ & $<.10$ & $<.10$ \\
\hline & & $\mathrm{R}$ & $10 / 26 / 02$ & 1300 & $<.10$ & $<.10$ & $<.10$ \\
\hline \multirow[t]{5}{*}{03219500} & Scioto River near Prospect & $\mathrm{R}$ & 06/04/02 & 1230 & .58 & .55 & $<.10$ \\
\hline & & LD & 06/04/02 & 1230 & .59 & .51 & $<.10$ \\
\hline & & FR & 06/04/02 & 1231 & .65 & .67 & $<.10$ \\
\hline & & $\mathrm{R}$ & 07/31/02 & 0930 & .48 & .82 & $<.10$ \\
\hline & & $\mathrm{R}$ & $10 / 01 / 02$ & 1030 & .28 & .47 & $<.10$ \\
\hline \multirow[t]{3}{*}{03223000} & Olentangy River at Claridon & $\mathrm{R}$ & 06/04/02 & 1100 & .24 & .27 & $<.10$ \\
\hline & & $\mathrm{R}$ & 07/31/02 & 1100 & $<.10$ & .34 & $<.10$ \\
\hline & & $\mathrm{R}$ & $10 / 26 / 02$ & 1500 & $<.10$ & .13 & $<.10$ \\
\hline \multirow[t]{4}{*}{03230500} & Big Darby Creek at Darbyville & $\mathrm{R}$ & 06/03/02 & 1200 & .60 & .31 & $<.10$ \\
\hline & & $\mathrm{R}$ & 08/01/02 & 0930 & $<.10$ & .25 & $<.10$ \\
\hline & & LD & 08/01/02 & 0930 & $<.10$ & .32 & $<.10$ \\
\hline & & $\mathrm{R}$ & $10 / 01 / 02$ & 1105 & .25 & .34 & $<.10$ \\
\hline \multirow[t]{4}{*}{03234500} & Scioto River at Higby & $\mathrm{R}$ & 06/04/02 & 1015 & .24 & .36 & $<.10$ \\
\hline & & $\mathrm{R}$ & 07/30/02 & 1100 & .23 & .94 & $<.10$ \\
\hline & & $\mathrm{R}$ & $10 / 28 / 02$ & 1000 & .28 & .89 & $<.10$ \\
\hline & & LD & $10 / 28 / 02$ & 1000 & .29 & .99 & $<.10$ \\
\hline 03240000 & Little Miami River near Oldtown & $\mathrm{R}$ & 06/05/02 & 1320 & $<.10$ & $<.10$ & $<.10$ \\
\hline
\end{tabular}


Table 6. Analytical results for glyphosate, aminomethylphosphonic acid, and glufosinate in Toxic Substances Hydrology Program studies, 2001-06.— Continued

[Sample type: FB, field blank; FR, field replicate; R, regular; LD, laboratory duplicate; --, no data; <, less than; $\mu \mathrm{g} / \mathrm{L}$, micrograms per liter]

\begin{tabular}{|c|c|c|c|c|c|c|c|}
\hline \multirow[b]{2}{*}{ Site number } & \multirow[b]{2}{*}{ Site location } & \multirow[b]{2}{*}{$\begin{array}{l}\text { Sam- } \\
\text { ple } \\
\text { type }\end{array}$} & \multirow[b]{2}{*}{$\begin{array}{c}\text { Date of } \\
\text { collection } \\
\text { (month/day/ } \\
\text { year) }\end{array}$} & \multirow[b]{2}{*}{$\begin{array}{l}\text { Col- } \\
\text { lec- } \\
\text { tion } \\
\text { time } \\
\text { (24- } \\
\text { hour) }\end{array}$} & \multicolumn{3}{|c|}{ Concentrations } \\
\hline & & & & & $\begin{array}{l}\text { Glypho- } \\
\text { sate } \\
\text { ( } \mu \mathrm{g} / \mathrm{L})\end{array}$ & 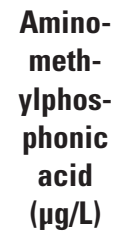 & $\begin{array}{c}\text { Glufos- } \\
\text { inate } \\
(\mu \mathrm{g} / \mathrm{L})\end{array}$ \\
\hline \multicolumn{8}{|c|}{ Reconnaissance data for glyphosate in nine Midwestern States-Continued } \\
\hline \multicolumn{8}{|c|}{ Ohio-Continued } \\
\hline \multirow[t]{2}{*}{03240000} & Little Miami River near Oldtown-Continued & $\mathrm{R}$ & 07/17/02 & 1200 & $<0.10$ & $<0.10$ & $<0.10$ \\
\hline & & $\mathrm{R}$ & $10 / 27 / 2002$ & 0700 & .45 & .17 & $<.10$ \\
\hline \multirow[t]{4}{*}{032667900} & Mad River at St. Paris Pike at Eagle City & $\mathrm{R}$ & $06 / 05 / 02$ & 1130 & $<.10$ & .11 & $<.10$ \\
\hline & & $\mathrm{R}$ & 07/17/02 & 1030 & $<.10$ & $<.10$ & $<.10$ \\
\hline & & FR & 07/17/02 & 1031 & $<.10$ & $<.10$ & $<.10$ \\
\hline & & $\mathrm{R}$ & $10 / 27 / 2002$ & 0800 & $<.10$ & $<.10$ & $<.10$ \\
\hline \multirow[t]{4}{*}{04185000} & Tiffin River at Stryker & $\mathrm{R}$ & $05 / 29 / 02$ & 1700 & .16 & $<.10$ & $<.10$ \\
\hline & & $\mathrm{R}$ & $07 / 23 / 02$ & 1530 & $<.10$ & .26 & $<.10$ \\
\hline & & $\mathrm{R}$ & $11 / 06 / 02$ & 1200 & $<.10$ & $<.10$ & $<.10$ \\
\hline & & FR & $11 / 06 / 02$ & 1201 & $<.10$ & $<.10$ & $<.10$ \\
\hline \multirow[t]{3}{*}{04186500} & Auglaize River near Fort Jennings & $\mathrm{R}$ & $05 / 28 / 02$ & 1115 & $<.10$ & .28 & $<.10$ \\
\hline & & $\mathrm{R}$ & $07 / 23 / 02$ & 1100 & .17 & .58 & $<.10$ \\
\hline & & $\mathrm{R}$ & $10 / 30 / 02$ & 1300 & $<.10$ & .30 & $<.10$ \\
\hline \multicolumn{8}{|c|}{ Wisconsin } \\
\hline \multirow[t]{5}{*}{04087240} & Root River at Racine & $\mathrm{R}$ & 06/03/02 & 1350 & $<.10$ & .10 & $<.10$ \\
\hline & & FR & 06/03/02 & 1351 & $<.10$ & .18 & $<.10$ \\
\hline & & $\mathrm{R}$ & $07 / 26 / 02$ & 1130 & $<.10$ & .29 & $<.10$ \\
\hline & & LD & $07 / 26 / 02$ & 1130 & $<.10$ & .23 & $<.10$ \\
\hline & & $\mathrm{R}$ & 09/03/02 & 1110 & .12 & .35 & $<.10$ \\
\hline \multirow[t]{5}{*}{05340500} & St. Croix River at St Croix Falls & $\mathrm{R}$ & $06 / 20 / 02$ & 1130 & $<.10$ & $<.10$ & $<.10$ \\
\hline & & $\mathrm{R}$ & $06 / 27 / 02$ & 1130 & $<.10$ & $<.10$ & $<.10$ \\
\hline & & $\mathrm{LD}$ & $06 / 27 / 02$ & 1130 & $<.10$ & $<.10$ & $<.10$ \\
\hline & & $\mathrm{R}$ & 09/03/02 & 1520 & $<.10$ & $<.10$ & $<.10$ \\
\hline & & LD & 09/03/02 & 1520 & $<.10$ & $<.10$ & $<.10$ \\
\hline \multirow[t]{3}{*}{05407000} & Wisconsin River at Muscoda & $\mathrm{R}$ & $05 / 28 / 02$ & 1330 & $<.10$ & $<.10$ & $<.10$ \\
\hline & & $\mathrm{R}$ & $06 / 27 / 02$ & 1215 & $<.10$ & $<.10$ & $<.10$ \\
\hline & & $\mathrm{R}$ & $09 / 23 / 02$ & 1130 & $<.10$ & $<.10$ & $<.10$ \\
\hline
\end{tabular}


Table 6. Analytical results for glyphosate, aminomethylphosphonic acid, and glufosinate in Toxic Substances Hydrology Program studies, 2001-06.Continued

[Sample type: FB, field blank; FR, field replicate; R, regular; LD, laboratory duplicate; --, no data; <, less than; $\mu \mathrm{g} / \mathrm{L}$, micrograms per liter]

\begin{tabular}{|c|c|c|c|c|c|c|c|}
\hline \multirow[b]{2}{*}{ Site number } & \multirow[b]{2}{*}{ Site location } & \multirow[b]{2}{*}{$\begin{array}{l}\text { Sam- } \\
\text { ple } \\
\text { type }\end{array}$} & \multirow[b]{2}{*}{$\begin{array}{c}\text { Date of } \\
\text { collection } \\
\text { (month/day/ } \\
\text { year) }\end{array}$} & \multirow[b]{2}{*}{$\begin{array}{l}\text { Col- } \\
\text { lec- } \\
\text { tion } \\
\text { time } \\
\text { (24- } \\
\text { hour) }\end{array}$} & \multicolumn{3}{|c|}{ Concentrations } \\
\hline & & & & & $\begin{array}{l}\text { Glypho- } \\
\text { sate } \\
\text { ( } \mu \mathrm{g} / \mathrm{L})\end{array}$ & $\begin{array}{l}\text { Amino- } \\
\text { meth- } \\
\text { ylphos- } \\
\text { phonic } \\
\text { acid } \\
\text { ( } \mu \mathrm{g} / \mathrm{L})\end{array}$ & $\begin{array}{c}\text { Glufos- } \\
\text { inate } \\
(\mu \mathrm{g} / \mathrm{L})\end{array}$ \\
\hline \multicolumn{8}{|c|}{ Reconnaissance data for glyphosate in nine Midwestern States-Continued } \\
\hline \multicolumn{8}{|c|}{ Wisconsin-Continued } \\
\hline \multirow[t]{4}{*}{05430500} & Rock River at Afton & $\mathrm{R}$ & 06/04/02 & 1300 & $<0.10$ & 0.16 & $<0.10$ \\
\hline & & $\mathrm{R}$ & 07/11/02 & 1229 & $<.10$ & .33 & $<.10$ \\
\hline & & FB & 07/11/02 & 1230 & $<.10$ & $<.10$ & $<.10$ \\
\hline & & $\mathrm{R}$ & 09/20/02 & 0945 & $<.10$ & .41 & $<.10$ \\
\hline \multicolumn{8}{|c|}{ Mississippi River glyphosate study, 2001-03 } \\
\hline \multirow[t]{25}{*}{07374000} & Mississippi River at Baton Rouge, Louisiana & $\mathrm{R}$ & $10 / 31 / 01$ & 1300 & $<.10$ & .18 & $<.10$ \\
\hline & & $\mathrm{R}$ & $11 / 14 / 01$ & 1300 & $<.10$ & .18 & $<.10$ \\
\hline & & $\mathrm{R}$ & $11 / 28 / 01$ & 1200 & $<.10$ & .23 & $<.10$ \\
\hline & & $\mathrm{R}$ & $12 / 10 / 01$ & 1300 & $<.10$ & .14 & $<.10$ \\
\hline & & LD & $12 / 10 / 01$ & 1300 & $<.10$ & .11 & $<.10$ \\
\hline & & $\mathrm{R}$ & 01/03/02 & 1140 & $<.10$ & .15 & $<.10$ \\
\hline & & LD & 01/03/02 & 1140 & $<.10$ & .10 & $<.10$ \\
\hline & & $\mathrm{R}$ & 01/17/02 & 1100 & $<.10$ & .12 & $<.10$ \\
\hline & & LD & 01/17/02 & 1100 & $<.10$ & .13 & $<.10$ \\
\hline & & $\mathrm{R}$ & 02/04/02 & 1300 & $<.10$ & .15 & $<.10$ \\
\hline & & LD & $02 / 04 / 02$ & 1300 & $<.10$ & .14 & $<.10$ \\
\hline & & $\mathrm{R}$ & $02 / 28 / 02$ & 1230 & $<.10$ & .15 & $<.10$ \\
\hline & & LD & $02 / 28 / 02$ & 1230 & $<.10$ & .18 & $<.10$ \\
\hline & & $\mathrm{R}$ & 03/11/02 & 1300 & $<.10$ & .24 & $<.10$ \\
\hline & & $\mathrm{R}$ & 03/28/02 & 1300 & $<.10$ & .38 & $<.10$ \\
\hline & & $\mathrm{R}$ & $04 / 12 / 02$ & 1115 & $<.10$ & $<.10$ & $<.10$ \\
\hline & & $\mathrm{R}$ & 05/02/02 & 1300 & $<.10$ & $<.10$ & $<.10$ \\
\hline & & $\mathrm{R}$ & $05 / 15 / 02$ & 1300 & $<.10$ & .12 & $<.10$ \\
\hline & & $\mathrm{R}$ & 06/05/02 & 1200 & $<.10$ & .16 & $<.10$ \\
\hline & & $\mathrm{R}$ & 06/11/02 & 1030 & $<.10$ & .16 & $<.10$ \\
\hline & & $\mathrm{R}$ & 07/02/02 & 1130 & $<.10$ & .17 & $<.10$ \\
\hline & & $\mathrm{R}$ & $07 / 25 / 02$ & 1400 & $<.10$ & .21 & $<.10$ \\
\hline & & $\mathrm{R}$ & 08/21/02 & 1100 & $<.10$ & .21 & $<.10$ \\
\hline & & $\mathrm{R}$ & 09/17/02 & 1400 & $<.10$ & .27 & $<.10$ \\
\hline & & LD & 09/17/02 & 1400 & $<.10$ & .22 & $<.10$ \\
\hline
\end{tabular}


Table 6. Analytical results for glyphosate, aminomethylphosphonic acid, and glufosinate in Toxic Substances Hydrology Program studies, 2001-06.— Continued

[Sample type: FB, field blank; FR, field replicate; R, regular; LD, laboratory duplicate; --, no data; <, less than; $\mu \mathrm{g} / \mathrm{L}$, micrograms per liter]

\begin{tabular}{|c|c|c|c|c|c|c|c|}
\hline \multirow[b]{2}{*}{ Site number } & \multirow[b]{2}{*}{ Site location } & \multirow[b]{2}{*}{$\begin{array}{c}\text { Sam- } \\
\text { ple } \\
\text { type }\end{array}$} & \multirow[b]{2}{*}{$\begin{array}{c}\text { Date of } \\
\text { collection } \\
\text { (month/day/ } \\
\text { year) }\end{array}$} & \multirow[b]{2}{*}{$\begin{array}{l}\text { Col- } \\
\text { lec- } \\
\text { tion } \\
\text { time } \\
\text { (24- } \\
\text { hour) }\end{array}$} & \multicolumn{3}{|c|}{ Concentrations } \\
\hline & & & & & $\begin{array}{c}\text { Glypho- } \\
\text { sate } \\
\text { ( } \mu \mathrm{g} / \mathrm{L})\end{array}$ & $\begin{array}{l}\text { Amino- } \\
\text { meth- } \\
\text { ylphos- } \\
\text { phonic } \\
\text { acid } \\
\text { ( } \mathrm{gg} / \mathrm{L} \text { ) }\end{array}$ & $\begin{array}{c}\text { Glufos- } \\
\text { inate } \\
(\mu \mathrm{g} / \mathrm{L})\end{array}$ \\
\hline \multicolumn{8}{|c|}{ Mississippi River glyphosate study, 2001-03-Continued } \\
\hline \multirow[t]{20}{*}{07374000} & \multirow{20}{*}{$\begin{array}{l}\text { Mississippi River at Baton Rouge, Louisiana- } \\
\text { Continued }\end{array}$} & $\mathrm{R}$ & $10 / 16 / 02$ & 1500 & $<0.10$ & 0.24 & $<0.10$ \\
\hline & & $\mathrm{R}$ & $11 / 19 / 02$ & 1400 & $<.10$ & .12 & $<.10$ \\
\hline & & $\mathrm{LD}$ & $11 / 19 / 02$ & 1400 & $<.10$ & .13 & $<.10$ \\
\hline & & $\mathrm{R}$ & $12 / 18 / 02$ & 1330 & $<.10$ & .10 & $<.10$ \\
\hline & & $\mathrm{R}$ & $01 / 15 / 03$ & 1400 & $<.10$ & $<.10$ & $<.10$ \\
\hline & & $\mathrm{R}$ & $02 / 20 / 03$ & 1330 & $<.10$ & .12 & $<.10$ \\
\hline & & LD & 02/20/03 & 1330 & $<.10$ & .17 & $<.10$ \\
\hline & & $\mathrm{R}$ & 03/13/03 & 1200 & $<.10$ & .11 & $<.10$ \\
\hline & & $\mathrm{R}$ & $04 / 03 / 03$ & 1100 & $<.10$ & .20 & $<.10$ \\
\hline & & $\mathrm{R}$ & 05/19/03 & 1330 & $<.10$ & .18 & $<.10$ \\
\hline & & LD & 05/19/03 & 1330 & $<.10$ & .20 & $<.10$ \\
\hline & & $\mathrm{R}$ & 06/03/03 & 1230 & $<.10$ & .19 & $<.10$ \\
\hline & & $\mathrm{R}$ & 06/19/03 & 0830 & $<.10$ & .20 & $<.10$ \\
\hline & & $\mathrm{R}$ & 07/03/03 & 1330 & $<.10$ & $<.10$ & $<.10$ \\
\hline & & $\mathrm{R}$ & $07 / 15 / 03$ & 1230 & $<.10$ & .31 & $<.10$ \\
\hline & & $\mathrm{R}$ & 07/30/03 & 1130 & $<.10$ & .31 & $<.10$ \\
\hline & & $\mathrm{R}$ & 09/17/03 & 1400 & $<.10$ & .30 & $<.10$ \\
\hline & & $\mathrm{R}$ & 09/29/03 & 1300 & $<.10$ & .33 & $<.10$ \\
\hline & & $\mathrm{R}$ & $10 / 15 / 03$ & 1430 & $<.10$ & .27 & $<.10$ \\
\hline & & LD & $10 / 15 / 03$ & 1430 & $<.10$ & .31 & $<.10$ \\
\hline \multicolumn{8}{|c|}{ Sensitive environments study, 2004-06 } \\
\hline \multicolumn{8}{|c|}{ District of Columbia } \\
\hline \multirow[t]{3}{*}{$\mathrm{C} \& \mathrm{O} 1$} & Carderock Vernal Pool & $\mathrm{R}$ & 03/08/06 & 1230 & $<.02$ & $<.02$ & $<.02$ \\
\hline & & LD & 03/08/06 & 1230 & $<.02$ & $<.02$ & $<.02$ \\
\hline & & $\mathrm{R}$ & 04/05/06 & 1245 & $<.02$ & $<.02$ & $<.02$ \\
\hline \multirow[t]{2}{*}{$\mathrm{C} \& \mathrm{O} 2$} & Lock 7 Vernal Pool & $\mathrm{R}$ & 03/08/06 & 1300 & $<.02$ & $<.02$ & $<.02$ \\
\hline & & $\mathrm{R}$ & 04/05/06 & 1330 & $<.02$ & $<.02$ & $<.02$ \\
\hline \multirow[t]{2}{*}{$\mathrm{C} \& \mathrm{O} 3$} & C\&O Canal at Lock 7 & $\mathrm{R}$ & 03/08/06 & 1330 & $<.02$ & $<.02$ & $<.02$ \\
\hline & & $\mathrm{R}$ & 04/05/06 & 1345 & .06 & $<.02$ & $<.02$ \\
\hline
\end{tabular}


Table 6. Analytical results for glyphosate, aminomethylphosphonic acid, and glufosinate in Toxic Substances Hydrology Program studies, 2001-06. Continued

[Sample type: FB, field blank; FR, field replicate; R, regular; LD, laboratory duplicate; --, no data; <, less than; $\mu \mathrm{g} / \mathrm{L}$, micrograms per liter]

\begin{tabular}{|c|c|c|c|c|c|c|c|}
\hline \multirow[b]{2}{*}{ Site number } & \multirow[b]{2}{*}{ Site location } & \multirow[b]{2}{*}{$\begin{array}{c}\text { Sam- } \\
\text { ple } \\
\text { type }\end{array}$} & \multirow[b]{2}{*}{$\begin{array}{c}\text { Date of } \\
\text { collection } \\
\text { (month/day/ } \\
\text { year) }\end{array}$} & \multirow[b]{2}{*}{$\begin{array}{l}\text { Col- } \\
\text { lec- } \\
\text { tion } \\
\text { time } \\
\text { (24- } \\
\text { hour) }\end{array}$} & \multicolumn{3}{|c|}{ Concentrations } \\
\hline & & & & & $\begin{array}{c}\text { Glypho- } \\
\text { sate } \\
\text { ( } \mu \mathrm{g} / \mathrm{L} \text { ) }\end{array}$ & $\begin{array}{c}\text { Amino- } \\
\text { meth- } \\
\text { ylphos- } \\
\text { phonic } \\
\text { acid } \\
\text { ( } \mu \mathrm{g} / \mathrm{L})\end{array}$ & $\begin{array}{c}\text { Glufos- } \\
\text { inate } \\
(\mu \mathrm{g} / \mathrm{L})\end{array}$ \\
\hline \multicolumn{8}{|c|}{ Sensitive environments study, 2004-06-Continued } \\
\hline \multicolumn{8}{|c|}{ District of Columbia-Continued } \\
\hline $\mathrm{C} \& \mathrm{O} 3$ & C\&O Canal at Lock 7-Continued & FR & $04 / 05 / 06$ & 1450 & $<0.02$ & $<0.02$ & $<0.02$ \\
\hline \multirow[t]{4}{*}{$\mathrm{RC} 1$} & Weir Pond at RCNP & $\mathrm{R}$ & $03 / 14 / 05$ & 1000 & $<.02$ & $<.02$ & $<.02$ \\
\hline & & $\mathrm{R}$ & $03 / 24 / 05$ & 0900 & $<.02$ & $<.02$ & $<.02$ \\
\hline & & $\mathrm{R}$ & 03/08/06 & 1615 & $<.02$ & $<.02$ & $<.02$ \\
\hline & & $\mathrm{R}$ & $04 / 05 / 06$ & 1500 & $<.02$ & $<.02$ & $<.02$ \\
\hline \multirow[t]{4}{*}{$\mathrm{RC} 3$} & Riley Spring Pond at RCNP & $\mathrm{R}$ & $03 / 14 / 05$ & 1100 & $<.02$ & $<.02$ & $<.02$ \\
\hline & & $\mathrm{LD}$ & $03 / 14 / 05$ & 1100 & $<.02$ & $<.02$ & $<.02$ \\
\hline & & $\mathrm{R}$ & $03 / 24 / 05$ & 1030 & 328 & 15 & $<.02$ \\
\hline & & FR & $03 / 24 / 05$ & 1031 & 301 & 41 & $<.02$ \\
\hline \multirow[t]{4}{*}{$\mathrm{RC} 4$} & Rock Creek at Riley Spring Pond & $\mathrm{R}$ & $03 / 14 / 05$ & 1200 & 1.1 & .21 & $<.02$ \\
\hline & & $\mathrm{R}$ & $03 / 24 / 05$ & 1100 & .60 & $<.02$ & $<.02$ \\
\hline & & $\mathrm{R}$ & 03/08/06 & 1530 & $<.02$ & .09 & $<.02$ \\
\hline & & $\mathrm{R}$ & 04/05/06 & 1520 & .12 & .12 & $<.02$ \\
\hline \multicolumn{8}{|c|}{ lowa } \\
\hline \multirow[t]{3}{*}{ DS1 } & Fieldside Wetlands at DNWR & $\mathrm{R}$ & $04 / 13 / 05$ & 1115 & $<.02$ & $<.02$ & $<.02$ \\
\hline & & $\mathrm{LD}$ & $04 / 13 / 05$ & 1115 & $<.02$ & $<.02$ & $<.02$ \\
\hline & & $\mathrm{R}$ & 06/08/05 & 1200 & 12 & 3.2 & $<.02$ \\
\hline \multirow[t]{5}{*}{ DS2 } & Rands Ditch at DNWR & $\mathrm{R}$ & $04 / 13 / 05$ & 1130 & .05 & .09 & $<.02$ \\
\hline & & $\mathrm{R}$ & 06/08/05 & 1110 & .12 & .18 & $<.02$ \\
\hline & & LD & 06/08/05 & 1110 & .10 & .17 & $<.02$ \\
\hline & & $\mathrm{R}$ & $05 / 24 / 06$ & 0840 & 1.5 & 2.9 & $<.02$ \\
\hline & & LD & $05 / 24 / 06$ & 0840 & 1.4 & 2.9 & $<.02$ \\
\hline \multirow[t]{3}{*}{ DS3 } & Browns Ditch at DNWR & $\mathrm{R}$ & $04 / 13 / 05$ & 1145 & $<.02$ & 1.0 & $<.02$ \\
\hline & & $\mathrm{R}$ & 06/08/05 & 1225 & .56 & .75 & $<.02$ \\
\hline & & $\mathrm{R}$ & $05 / 24 / 06$ & 0920 & $<.02$ & $<.02$ & $<.02$ \\
\hline \multirow[t]{2}{*}{ DS4 } & Browns Pond at DNWR & $\mathrm{R}$ & $04 / 13 / 05$ & 1200 & .05 & $<.02$ & $<.02$ \\
\hline & & $\mathrm{R}$ & 06/08/05 & 1230 & .09 & $<.02$ & $<.02$ \\
\hline
\end{tabular}


Table 6. Analytical results for glyphosate, aminomethylphosphonic acid, and glufosinate in Toxic Substances Hydrology Program studies, 2001-06. Continued

[Sample type: FB, field blank; FR, field replicate; R, regular; LD, laboratory duplicate; --, no data; <, less than; $\mu \mathrm{g} / \mathrm{L}$, micrograms per liter]

\begin{tabular}{|c|c|c|c|c|c|c|c|}
\hline \multirow[b]{2}{*}{ Site number } & \multirow[b]{2}{*}{ Site location } & \multirow[b]{2}{*}{$\begin{array}{c}\text { Sam- } \\
\text { ple } \\
\text { type }\end{array}$} & \multirow[b]{2}{*}{$\begin{array}{c}\text { Date of } \\
\text { collection } \\
\text { (month/day/ } \\
\text { year) }\end{array}$} & \multirow[b]{2}{*}{$\begin{array}{c}\text { Col- } \\
\text { lec- } \\
\text { tion } \\
\text { time } \\
\text { (24- } \\
\text { hour) }\end{array}$} & \multicolumn{3}{|c|}{ Concentrations } \\
\hline & & & & & $\begin{array}{c}\text { Glypho- } \\
\text { sate } \\
(\mu \mathrm{g} / \mathrm{L})\end{array}$ & $\begin{array}{c}\text { Amino- } \\
\text { meth- } \\
\text { ylphos- } \\
\text { phonic } \\
\text { acid } \\
\text { ( } \mathrm{g} / \mathrm{L})\end{array}$ & $\begin{array}{c}\text { Glufos- } \\
\text { inate } \\
\text { ( } \mu \mathrm{g} / \mathrm{L})\end{array}$ \\
\hline \multicolumn{8}{|c|}{ Sensitive environments study, 2004-06-Continued } \\
\hline \multicolumn{8}{|c|}{ lowa-Continued } \\
\hline DS5 & East Bend Pond at DNWR & $\mathrm{R}$ & $06 / 08 / 05$ & 1145 & $<0.02$ & $<0.02$ & $<0.02$ \\
\hline \multicolumn{8}{|c|}{ Michigan } \\
\hline \multirow[t]{2}{*}{ WYMO-10 } & Lake RSL near Saginaw & $\mathrm{R}$ & $08 / 12 / 05$ & 1000 & .04 & $<.02$ & $<.02$ \\
\hline & & LD & $08 / 12 / 05$ & 1000 & $<.02$ & $<.02$ & $<.02$ \\
\hline \multicolumn{8}{|c|}{ Nebraska } \\
\hline \multirow[t]{2}{*}{06880000} & Lincoln Creek near Seward & $\mathrm{R}$ & 07/09/04 & 1310 & 1.4 & 1.5 & $<.02$ \\
\hline & & $\mathrm{R}$ & 08/05/04 & 1100 & .26 & .97 & $<.02$ \\
\hline \multirow[t]{3}{*}{ TH1080 } & Creek at Field 413 & $\mathrm{R}$ & 07/09/04 & 1125 & 21 & 5.8 & $<.02$ \\
\hline & & $\mathrm{R}$ & 08/05/04 & 1430 & $<.02$ & .03 & $<.02$ \\
\hline & & $\mathrm{R}$ & 08/06/04 & 1100 & $<.02$ & .02 & $<.02$ \\
\hline \multirow[t]{2}{*}{ TH1090 } & Pond at Field 413 & $\mathrm{R}$ & 07/09/04 & 1200 & .08 & .21 & $<.02$ \\
\hline & & $\mathrm{R}$ & 08/05/04 & 1400 & 1.2 & 1.2 & $<.02$ \\
\hline WYMI112 & Frenchman Creek near Champion & $\mathrm{R}$ & 08/05/04 & 1045 & $<.02$ & $<.02$ & $<.02$ \\
\hline WYMI113 & Pond near Champion & $\mathrm{R}$ & $08 / 12 / 04$ & 0845 & 1.1 & .02 & $<.02$ \\
\hline WYMI114 & Rock Creek near Parks & $\mathrm{R}$ & $08 / 12 / 04$ & 0857 & $<.02$ & $<.02$ & $<.02$ \\
\hline WYMI19 & Pond near Republican River near Stratton & $\mathrm{R}$ & $07 / 21 / 04$ & 2130 & .25 & .12 & $<.02$ \\
\hline WYMI33 & Pond near Republican River near Franklin & $\mathrm{R}$ & $07 / 22 / 04$ & 2040 & $<.02$ & .08 & $<.02$ \\
\hline WYMI46 & Elkhorn River near Nickerson & $\mathrm{R}$ & $07 / 25 / 04$ & 2015 & .16 & .51 & $<.02$ \\
\hline \multicolumn{8}{|c|}{ New York } \\
\hline WYMO-09 & Lake Champlain near Plattsburg & $\mathrm{R}$ & $08 / 10 / 05$ & 0800 & .07 & .29 & $<.02$ \\
\hline \multicolumn{8}{|c|}{ South Dakota } \\
\hline \multirow[t]{2}{*}{ WYMI104 } & North Fork Rapid Creek near Silver City & $\mathrm{R}$ & 08/04/04 & 1945 & $<.02$ & $<.02$ & $<.02$ \\
\hline & & $\mathrm{LD}$ & 08/04/04 & 1945 & $<.02$ & $<.02$ & $<.02$ \\
\hline WYMI67 & North Bend Creek & $\mathrm{R}$ & $07 / 29 / 04$ & 1416 & $<.02$ & $<.02$ & $<.02$ \\
\hline WYMI83 & Big Sioux River near Egan & $\mathrm{R}$ & 07/31/04 & 2149 & $<.02$ & $<.02$ & $<.02$ \\
\hline WYMI91 & Buffalo Lakes near Lake City & $\mathrm{R}$ & 08/01/04 & 1005 & $<.02$ & $<.02$ & $<.02$ \\
\hline WYMI98 & Dry House Creek near Buffalo & $\mathrm{R}$ & $08 / 02 / 04$ & 1401 & $<.02$ & .04 & $<.02$ \\
\hline
\end{tabular}


Table 6. Analytical results for glyphosate, aminomethylphosphonic acid, and glufosinate in Toxic Substances Hydrology Program studies, 2001-06. Continued

[Sample type: FB, field blank; FR, field replicate; R, regular; LD, laboratory duplicate; --, no data; <, less than; $\mu \mathrm{g} / \mathrm{L}$, micrograms per liter]

\begin{tabular}{|c|c|c|c|c|c|c|c|}
\hline \multirow[b]{2}{*}{ Site number } & \multirow[b]{2}{*}{ Site location } & \multirow[b]{2}{*}{$\begin{array}{l}\text { Sam- } \\
\text { ple } \\
\text { type }\end{array}$} & \multirow[b]{2}{*}{$\begin{array}{c}\text { Date of } \\
\text { collection } \\
\text { (month/day/ } \\
\text { year) }\end{array}$} & \multirow[b]{2}{*}{$\begin{array}{l}\text { Col- } \\
\text { lec- } \\
\text { tion } \\
\text { time } \\
\text { (24- } \\
\text { hour) }\end{array}$} & \multicolumn{3}{|c|}{ Concentrations } \\
\hline & & & & & $\begin{array}{l}\text { Glypho- } \\
\text { sate } \\
\text { (pg/L) }\end{array}$ & $\begin{array}{c}\text { Amino- } \\
\text { meth- } \\
\text { ylphos- } \\
\text { phonic } \\
\text { acid } \\
\text { ( } \mu \mathrm{g} / \mathrm{L} \text { ) }\end{array}$ & $\begin{array}{c}\text { Glufos- } \\
\text { inate } \\
(\mu \mathrm{g} / \mathrm{L})\end{array}$ \\
\hline \multicolumn{8}{|c|}{ Sensitive environments study, 2004-06-Continued } \\
\hline \multicolumn{8}{|c|}{ Vermont } \\
\hline WYMO-06 & Lake Champlain near Swanton & $\mathrm{R}$ & 08/10/05 & 1200 & 0.04 & $<0.02$ & $<0.02$ \\
\hline \multirow[t]{2}{*}{ WYMO-08 } & Lake Champlain near Addison & $\mathrm{R}$ & 08/10/05 & 1600 & $<.02$ & .06 & $<.02$ \\
\hline & & LD & 08/10/05 & 1600 & $<.02$ & $<.02$ & $<.02$ \\
\hline \multicolumn{8}{|c|}{ Wyoming } \\
\hline \multirow[t]{15}{*}{ TH1010 } & Pond at Dugway Campground, Wyoming & $\mathrm{R}$ & 07/13/04 & 0800 & .02 & .27 & $<.02$ \\
\hline & & $\mathrm{R}$ & 07/18/04 & 0748 & $<.02$ & $<.02$ & $<.02$ \\
\hline & & $\mathrm{R}$ & 08/16/04 & 1115 & $<.02$ & $<.02$ & $<.02$ \\
\hline & & $\mathrm{R}$ & 04/19/05 & 0745 & $<.02$ & $<.02$ & $<.02$ \\
\hline & & LD & $04 / 19 / 05$ & 0745 & $<.02$ & $<.02$ & $<.02$ \\
\hline & & $\mathrm{R}$ & 05/05/05 & 1525 & $<.02$ & $<.02$ & $<.02$ \\
\hline & & LD & $05 / 05 / 05$ & 1525 & .02 & $<.02$ & $<.02$ \\
\hline & & $\mathrm{R}$ & $05 / 24 / 05$ & 0950 & .06 & $<.02$ & $<.02$ \\
\hline & & $\mathrm{R}$ & $05 / 31 / 05$ & 1545 & .02 & $<.02$ & $<.02$ \\
\hline & & $\mathrm{R}$ & $07 / 15 / 05$ & 0815 & $<.02$ & $<.02$ & $<.02$ \\
\hline & & $\mathrm{R}$ & 07/27/05 & 0800 & .08 & .06 & $<.02$ \\
\hline & & $\mathrm{R}$ & 08/09/05 & 0800 & .06 & .04 & $<.02$ \\
\hline & & $\mathrm{R}$ & $08 / 15 / 05$ & 0945 & $<.02$ & $<.02$ & $<.02$ \\
\hline & & $\mathrm{R}$ & $04 / 21 / 06$ & 0915 & $<.02$ & $<.02$ & $<.02$ \\
\hline & & $\mathrm{R}$ & 06/26/06 & 1020 & $<.02$ & $<.02$ & $<.02$ \\
\hline \multirow[t]{10}{*}{ TH1020 } & North Platte above Seminoe Reservation & $\mathrm{R}$ & 07/13/04 & 0745 & .04 & .66 & $<.02$ \\
\hline & & $\mathrm{R}$ & 07/18/04 & 0925 & $<.02$ & $<.02$ & $<.02$ \\
\hline & & $\mathrm{R}$ & 08/16/04 & 1330 & $<.02$ & $<.02$ & .05 \\
\hline & & $\mathrm{R}$ & $04 / 19 / 05$ & 0830 & $<.02$ & $<.02$ & $<.02$ \\
\hline & & $\mathrm{R}$ & 05/05/05 & 1505 & $<.02$ & $<.02$ & $<.02$ \\
\hline & & $\mathrm{R}$ & $05 / 24 / 05$ & 1040 & $<.02$ & $<.02$ & $<.02$ \\
\hline & & $\mathrm{R}$ & $05 / 31 / 05$ & 1530 & $<.02$ & $<.02$ & $<.02$ \\
\hline & & $\mathrm{R}$ & $07 / 15 / 05$ & 0745 & $<.02$ & $<.02$ & $<.02$ \\
\hline & & $\mathrm{R}$ & 08/09/05 & 1000 & .07 & .05 & $<.02$ \\
\hline & & $\mathrm{R}$ & $08 / 15 / 05$ & 1100 & $<.02$ & $<.02$ & $<.02$ \\
\hline
\end{tabular}


Table 6. Analytical results for glyphosate, aminomethylphosphonic acid, and glufosinate in Toxic Substances Hydrology Program studies, 2001-06.— Continued

[Sample type: FB, field blank; FR, field replicate; R, regular; LD, laboratory duplicate; --, no data; <, less than; $\mu \mathrm{g} / \mathrm{L}$, micrograms per liter]

\begin{tabular}{|c|c|c|c|c|c|c|c|}
\hline \multirow[b]{2}{*}{ Site number } & \multirow[b]{2}{*}{ Site location } & \multirow[b]{2}{*}{$\begin{array}{c}\text { Sam- } \\
\text { ple } \\
\text { type }\end{array}$} & \multirow[b]{2}{*}{$\begin{array}{c}\text { Date of } \\
\text { collection } \\
\text { (month/day/ } \\
\text { year) }\end{array}$} & \multirow[b]{2}{*}{$\begin{array}{l}\text { Col- } \\
\text { lec- } \\
\text { tion } \\
\text { time } \\
\text { (24- } \\
\text { hour) }\end{array}$} & \multicolumn{3}{|c|}{ Concentrations } \\
\hline & & & & & $\begin{array}{c}\text { Glypho- } \\
\text { sate } \\
\text { ( } \mu \mathrm{g} / \mathrm{L})\end{array}$ & 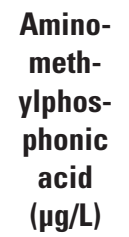 & $\begin{array}{c}\text { Glufos- } \\
\text { inate } \\
(\mu \mathrm{g} / \mathrm{L})\end{array}$ \\
\hline \multicolumn{8}{|c|}{ Sensitive environments study, 2004-06-Continued } \\
\hline \multicolumn{8}{|c|}{ Wyoming-Continued } \\
\hline \multirow[t]{2}{*}{ TH1020 } & North Platte above Seminoe Reservation-Continued & $\mathrm{R}$ & $04 / 21 / 06$ & 0930 & $<0.02$ & $<0.02$ & $<0.02$ \\
\hline & & $\mathrm{R}$ & $06 / 26 / 06$ & 1000 & $<.02$ & $<.02$ & $<.02$ \\
\hline \multicolumn{8}{|c|}{ Leary Weber Ditch Basin, Indiana (ground water) } \\
\hline 395023085492802 & ACTTR1-1-B piezometer at Mohawk & $\mathrm{R}$ & $06 / 29 / 04$ & 1015 & $<.02$ & .04 & $<.02$ \\
\hline \multirow[t]{2}{*}{395023085492804} & ACTTR1-1-D piezometer at Mohawk & FR & $06 / 29 / 04$ & 1045 & $<.02$ & .04 & $<.02$ \\
\hline & & $\mathrm{R}$ & $10 / 25 / 04$ & 1230 & .05 & .10 & $<.02$ \\
\hline \multirow[t]{2}{*}{395023085492810} & ACTTR1-1-2 piezometer at Mohawk & $\mathrm{R}$ & $06 / 28 / 04$ & 1600 & $<.02$ & .07 & $<.02$ \\
\hline & & $\mathrm{R}$ & $10 / 25 / 04$ & 1300 & .12 & .22 & $<.02$ \\
\hline 395023085492811 & ACTTR1-1-2-C piezometer at Mohawk & $\mathrm{R}$ & $06 / 28 / 04$ & 1645 & $<.02$ & .05 & $<.02$ \\
\hline 395023085492812 & ACTTR1-1-2-D piezometer at Mohawk & $\mathrm{R}$ & $10 / 25 / 04$ & 1330 & .17 & .22 & $<.02$ \\
\hline \multirow[t]{2}{*}{395023085492814} & ACTTR1-1-3-B piezometer at Mohawk & $\mathrm{R}$ & $06 / 29 / 04$ & 1400 & $<.02$ & .08 & $<.02$ \\
\hline & & $\mathrm{R}$ & $10 / 25 / 04$ & 1430 & .27 & .33 & $<.02$ \\
\hline \multirow[t]{2}{*}{395023085492816} & ACTTR1-1-3-D piezometer at Mohawk & $\mathrm{R}$ & $06 / 29 / 04$ & 1230 & $<.02$ & .08 & $<.02$ \\
\hline & & $\mathrm{R}$ & $10 / 25 / 04$ & 1500 & .12 & .17 & $<.02$ \\
\hline \multirow[t]{2}{*}{395023085492822} & ACTTR1-1-4-A piezometer at Mohawk & $\mathrm{R}$ & $06 / 28 / 04$ & 1230 & $<.02$ & .08 & $<.02$ \\
\hline & & $\mathrm{R}$ & $10 / 25 / 04$ & 1600 & .02 & .10 & $<.02$ \\
\hline \multirow[t]{4}{*}{395023085492824} & ACTTR1-1-4-C piezometer at Mohawk & $\mathrm{R}$ & $06 / 28 / 04$ & 1500 & $<.02$ & .07 & $<.02$ \\
\hline & & $\mathrm{R}$ & $10 / 25 / 04$ & 1200 & $<.02$ & .35 & $<.02$ \\
\hline & & $\mathrm{R}$ & $10 / 25 / 04$ & 1630 & $<.02$ & $<.02$ & $<.02$ \\
\hline & & FR & $10 / 25 / 04$ & 1631 & $<.02$ & $<.02$ & $<.02$ \\
\hline \multirow[t]{2}{*}{395023085492840} & ACTTR1-3-1-B piezometer at Mohawk & $\mathrm{R}$ & $06 / 29 / 04$ & 1530 & $<.02$ & .04 & $<.02$ \\
\hline & & $\mathrm{R}$ & $10 / 22 / 04$ & 1300 & $<.02$ & $<.02$ & $<.02$ \\
\hline \multirow[t]{2}{*}{395023085492842} & ACTTR1-3-1-D piezometer at Mohawk & $\mathrm{R}$ & $06 / 29 / 04$ & 1615 & $<.02$ & .06 & $<.02$ \\
\hline & & $\mathrm{R}$ & $10 / 22 / 04$ & 1330 & $<.02$ & $<.02$ & $<.02$ \\
\hline \multirow[t]{4}{*}{395023085492844} & ACTTR1-3-2-B piezometer at Mohawk & $\mathrm{R}$ & $06 / 29 / 04$ & 1745 & $<.02$ & .07 & $<.02$ \\
\hline & & $\mathrm{R}$ & $06 / 30 / 04$ & 1015 & $<.02$ & .06 & $<.02$ \\
\hline & & $\mathrm{R}$ & $10 / 22 / 04$ & 1100 & $<.02$ & $<.02$ & $<.02$ \\
\hline & & $\mathrm{R}$ & $10 / 22 / 04$ & 1400 & $<.02$ & .15 & $<.02$ \\
\hline
\end{tabular}


Table 6. Analytical results for glyphosate, aminomethylphosphonic acid, and glufosinate in Toxic Substances Hydrology Program studies, 2001-06. Continued

[Sample type: FB, field blank; FR, field replicate; R, regular; LD, laboratory duplicate; --, no data; <, less than; $\mu \mathrm{g} / \mathrm{L}$, micrograms per liter]

\begin{tabular}{|c|c|c|c|c|c|c|c|}
\hline \multirow[b]{2}{*}{ Site number } & \multirow[b]{2}{*}{ Site location } & \multirow[b]{2}{*}{$\begin{array}{c}\text { Sam- } \\
\text { ple } \\
\text { type }\end{array}$} & \multirow[b]{2}{*}{$\begin{array}{c}\text { Date of } \\
\text { collection } \\
\text { (month/day/ } \\
\text { year) }\end{array}$} & \multirow[b]{2}{*}{$\begin{array}{l}\text { Col- } \\
\text { lec- } \\
\text { tion } \\
\text { time } \\
\text { (24- } \\
\text { hour) }\end{array}$} & \multicolumn{3}{|c|}{ Concentrations } \\
\hline & & & & & $\begin{array}{c}\text { Glypho- } \\
\text { sate } \\
\text { ( } \mu \mathrm{g} / \mathrm{L} \text { ) }\end{array}$ & 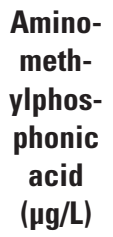 & $\begin{array}{c}\text { Glufos- } \\
\text { inate } \\
(\mu \mathrm{g} / \mathrm{L})\end{array}$ \\
\hline \multicolumn{8}{|c|}{ Sensitive environments study, 2004-06-Continued } \\
\hline \multicolumn{8}{|c|}{ Leary Weber Ditch Basin, Indiana (ground water)_Continued } \\
\hline \multirow[t]{2}{*}{395023085492846} & ACTTR1-3-2-D piezometer at Mohawk & $\mathrm{R}$ & $06 / 29 / 04$ & 1830 & $<0.02$ & 0.05 & $<0.02$ \\
\hline & & $\mathrm{R}$ & $10 / 22 / 04$ & 1200 & $<.02$ & $<.02$ & $<.02$ \\
\hline \multirow[t]{2}{*}{395023085492850} & ACTTR1-3-3-D piezometer at Mohawk & $\mathrm{R}$ & $06 / 30 / 04$ & 1100 & $<.02$ & .05 & $<.02$ \\
\hline & & $\mathrm{R}$ & $10 / 22 / 04$ & 1430 & $<.02$ & $<.02$ & $<.02$ \\
\hline \multirow[t]{4}{*}{395023085492853} & ACTTR1-3-4-A piezometer at Mohawk & FB & $06 / 30 / 04$ & 1258 & $<.02$ & $<.02$ & $<.02$ \\
\hline & & $\mathrm{R}$ & $06 / 30 / 04$ & 1300 & $<.02$ & .06 & $<.02$ \\
\hline & & FR & $06 / 30 / 04$ & 1301 & $<.02$ & .03 & $<.02$ \\
\hline & & $\mathrm{R}$ & $10 / 22 / 04$ & 1500 & $<.02$ & .09 & $<.02$ \\
\hline 395023085492822 & ACTTR1-3-4-A piezometer at Mohawk & FB & $10 / 25 / 04$ & 1608 & $<.02$ & $<.02$ & $<.02$ \\
\hline \multirow[t]{3}{*}{395023085492856} & ACTTR1-3-4-D piezometer at Mohawk & $\mathrm{R}$ & $06 / 30 / 04$ & 1430 & $<.02$ & .04 & $<.02$ \\
\hline & & FR & $06 / 30 / 04$ & 1431 & $<.02$ & .05 & $<.02$ \\
\hline & & $\mathrm{R}$ & $10 / 22 / 04$ & 1530 & $<.02$ & $<.02$ & $<.02$ \\
\hline \multirow[t]{16}{*}{395124085510801} & Tile drain ACTTD1-1 at Mohawk & $\mathrm{R}$ & $03 / 22 / 04$ & 1030 & $<.02$ & $<.02$ & $<.02$ \\
\hline & & $\mathrm{R}$ & $03 / 30 / 04$ & 1030 & $<.02$ & .07 & $<.02$ \\
\hline & & $\mathrm{R}$ & $05 / 15 / 04$ & 1335 & .28 & .09 & $<.02$ \\
\hline & & $\mathrm{R}$ & 05/19/04 & 0230 & 3.8 & 2.6 & $<.02$ \\
\hline & & $\mathrm{R}$ & $05 / 19 / 04$ & 0330 & 1.2 & .18 & $<.02$ \\
\hline & & $\mathrm{R}$ & 05/19/04 & 0440 & .74 & .69 & $<.02$ \\
\hline & & FR & 05/19/04 & 0441 & .93 & .19 & $<.02$ \\
\hline & & $\mathrm{R}$ & 05/19/04 & 1510 & .27 & .07 & $<.02$ \\
\hline & & $\mathrm{R}$ & $05 / 20 / 04$ & 1345 & .15 & .07 & $<.02$ \\
\hline & & $\mathrm{R}$ & $05 / 30 / 04$ & 2130 & 1.5 & .17 & $<.02$ \\
\hline & & $\mathrm{R}$ & $05 / 30 / 04$ & 2130 & 2.6 & .61 & $<.02$ \\
\hline & & $\mathrm{R}$ & $05 / 30 / 04$ & 2330 & 3.7 & .54 & $<.02$ \\
\hline & & $\mathrm{R}$ & $05 / 31 / 04$ & 0530 & 4.7 & 1.0 & $<.02$ \\
\hline & & $\mathrm{R}$ & $05 / 31 / 04$ & 1215 & 1.2 & 0.82 & $<0.02$ \\
\hline & & $\mathrm{R}$ & 06/01/04 & 1215 & .34 & .21 & $<.02$ \\
\hline & & $\mathrm{R}$ & 06/01/04 & 1216 & .32 & .20 & $<.02$ \\
\hline
\end{tabular}


Table 6. Analytical results for glyphosate, aminomethylphosphonic acid, and glufosinate in Toxic Substances Hydrology Program studies, 2001-06.— Continued

[Sample type: FB, field blank; FR, field replicate; R, regular; LD, laboratory duplicate; --, no data; <, less than; $\mu \mathrm{g} / \mathrm{L}$, micrograms per liter]

\begin{tabular}{|c|c|c|c|c|c|c|c|}
\hline \multirow[b]{2}{*}{ Site number } & \multirow[b]{2}{*}{ Site location } & \multirow[b]{2}{*}{$\begin{array}{l}\text { Sam- } \\
\text { ple } \\
\text { type }\end{array}$} & \multirow[b]{2}{*}{$\begin{array}{c}\text { Date of } \\
\text { collection } \\
\text { (month/day/ } \\
\text { year) }\end{array}$} & \multirow[b]{2}{*}{$\begin{array}{l}\text { Col- } \\
\text { lec- } \\
\text { tion } \\
\text { time } \\
\text { (24- } \\
\text { hour) }\end{array}$} & \multicolumn{3}{|c|}{ Concentrations } \\
\hline & & & & & $\begin{array}{l}\text { Glypho- } \\
\text { sate } \\
\text { ( } \mu \mathrm{g} / \mathrm{L})\end{array}$ & 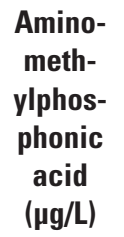 & $\begin{array}{c}\text { Glufos- } \\
\text { inate } \\
(\mu \mathrm{g} / \mathrm{L})\end{array}$ \\
\hline \multicolumn{8}{|c|}{ Sensitive environments study, 2004-06-Continued } \\
\hline \multicolumn{8}{|c|}{ Leary Weber Ditch Basin, Indiana (ground water)_Continued } \\
\hline \multirow[t]{9}{*}{395124085510801} & Tile drain ACTTD1-1 at Mohawk & $\mathrm{R}$ & 06/30/04 & 1745 & 0.08 & 0.15 & $<0.02$ \\
\hline & & FB & 08/26/04 & 1200 & $<.02$ & $<.02$ & $<.02$ \\
\hline & & $\mathrm{R}$ & 04/19/05 & 1545 & $<.02$ & .43 & $<.02$ \\
\hline & & $\mathrm{R}$ & 06/12/05 & 2100 & .04 & .25 & $<.02$ \\
\hline & & $\mathrm{R}$ & $06 / 12 / 05$ & 2200 & .04 & .19 & $<.02$ \\
\hline & & $\mathrm{R}$ & 06/12/05 & 2300 & $<.02$ & .16 & $<.02$ \\
\hline & & $\mathrm{R}$ & 06/13/05 & 0600 & .04 & .15 & $<.02$ \\
\hline & & $\mathrm{R}$ & 06/13/05 & 2200 & $<.02$ & .15 & $<.02$ \\
\hline & & $\mathrm{R}$ & 06/14/05 & 1130 & $<.02$ & .14 & $<.02$ \\
\hline \multirow[t]{4}{*}{395045085510930} & Vadose zone ACTVZ1-1A at Mohawk & $\mathrm{R}$ & $05 / 25 / 04$ & 1110 & $<.02$ & .06 & $<.02$ \\
\hline & & $\mathrm{R}$ & 06/22/04 & 1630 & $<.02$ & .09 & $<.02$ \\
\hline & & $\mathrm{R}$ & $10 / 20 / 04$ & 1145 & $<.02$ & $<.02$ & $<.02$ \\
\hline & & $\mathrm{R}$ & $06 / 22 / 05$ & 1130 & $<.02$ & .06 & $<.02$ \\
\hline \multirow[t]{5}{*}{395045085510931} & Vadose zone ACTVZ1-1B at Mohawk & $\mathrm{R}$ & 04/06/04 & 1100 & $<.02$ & .05 & $<.02$ \\
\hline & & $\mathrm{R}$ & $05 / 25 / 04$ & 1100 & $<.02$ & .05 & $<.02$ \\
\hline & & $\mathrm{R}$ & $06 / 22 / 04$ & 1640 & $<.02$ & .03 & $<.02$ \\
\hline & & $\mathrm{R}$ & $10 / 20 / 04$ & 1155 & $<.02$ & $<.02$ & $<.02$ \\
\hline & & $\mathrm{R}$ & $06 / 22 / 05$ & 1140 & .03 & $<.02$ & $<.02$ \\
\hline \multirow[t]{6}{*}{395045085510932} & Vadose zone ACTVZ1-1C at Mohawk & $\mathrm{R}$ & 04/06/04 & 1100 & .03 & .09 & $<.02$ \\
\hline & & $\mathrm{R}$ & $05 / 25 / 04$ & 1130 & $<.02$ & .08 & $<.02$ \\
\hline & & $\mathrm{R}$ & $06 / 22 / 04$ & 1650 & $<.02$ & .03 & $<.02$ \\
\hline & & $\mathrm{R}$ & 08/24/04 & 0925 & $<.02$ & $<.02$ & $<.02$ \\
\hline & & $\mathrm{R}$ & $10 / 20 / 04$ & 1205 & $<.02$ & $<.02$ & $<.02$ \\
\hline & & $\mathrm{R}$ & $06 / 22 / 05$ & 1150 & $<.02$ & $<.02$ & $<.02$ \\
\hline \multirow[t]{3}{*}{395045085510933} & Vadose zone ACTVZ1-1D at Mohawk (saturated) & $\mathrm{R}$ & $04 / 06 / 04$ & 1100 & .05 & .29 & $<.02$ \\
\hline & & $\mathrm{R}$ & $05 / 25 / 04$ & 1140 & .14 & .20 & $<.02$ \\
\hline & & $\mathrm{R}$ & $06 / 22 / 04$ & 1700 & .04 & .24 & $<.02$ \\
\hline
\end{tabular}


Table 6. Analytical results for glyphosate, aminomethylphosphonic acid, and glufosinate in Toxic Substances Hydrology Program studies, 2001-06. Continued

[Sample type: FB, field blank; FR, field replicate; R, regular; LD, laboratory duplicate; --, no data; <, less than; $\mu \mathrm{g} / \mathrm{L}$, micrograms per liter]

\begin{tabular}{|c|c|c|c|c|c|c|c|}
\hline \multirow[b]{2}{*}{ Site number } & \multirow[b]{2}{*}{ Site location } & \multirow[b]{2}{*}{$\begin{array}{l}\text { Sam- } \\
\text { ple } \\
\text { type }\end{array}$} & \multirow[b]{2}{*}{$\begin{array}{c}\text { Date of } \\
\text { collection } \\
\text { (month/day/ } \\
\text { year) }\end{array}$} & \multirow[b]{2}{*}{$\begin{array}{l}\text { Col- } \\
\text { lec- } \\
\text { tion } \\
\text { time } \\
\text { (24- } \\
\text { hour) }\end{array}$} & \multicolumn{3}{|c|}{ Concentrations } \\
\hline & & & & & $\begin{array}{c}\text { Glypho- } \\
\text { sate } \\
\text { ( } \mu \mathrm{g} / \mathrm{L})\end{array}$ & $\begin{array}{l}\text { Amino- } \\
\text { meth- } \\
\text { ylphos- } \\
\text { phonic } \\
\text { acid } \\
\text { ( } \mu \mathrm{g} / \mathrm{L})\end{array}$ & $\begin{array}{l}\text { Glufos- } \\
\text { inate } \\
(\mu \mathrm{g} / \mathrm{L})\end{array}$ \\
\hline \multicolumn{8}{|c|}{ Sensitive environments study, 2004-06-Continued } \\
\hline \multicolumn{8}{|c|}{ Leary Weber Ditch Basin, Indiana (ground water)_Continued } \\
\hline 395045085510933 & Vadose zone ACTVZ1-1D at Mohawk (unsaturated) & $\mathrm{R}$ & $10 / 20 / 04$ & 1215 & $<0.02$ & $<0.02$ & $<0.02$ \\
\hline & & $\mathrm{R}$ & 06/22/05 & 1200 & $<.02$ & $<.02$ & $<.02$ \\
\hline 395151085504540 & Vadose zone ACTVZ1-2F at Mohawk (unsaturated) & $\mathrm{R}$ & $05 / 25 / 04$ & 1000 & .02 & .17 & $<.02$ \\
\hline 395151085504541 & Vadose zone ACTVZ1-2F at Mohawk (unsaturated) & $\mathrm{R}$ & $05 / 25 / 04$ & 1010 & .02 & .14 & $<.02$ \\
\hline 395151085504530 & Vadose zone ACTVZ1-2A at Mohawk (saturated) & $\mathrm{R}$ & $05 / 25 / 04$ & 1015 & $<.02$ & .06 & $<.02$ \\
\hline 395151085504530 & Vadose zone ACTVZ1-2A at Mohawk (saturated) & $\mathrm{R}$ & $06 / 22 / 04$ & 1200 & $<.02$ & .08 & $<.02$ \\
\hline 395151085504530 & Vadose zone ACTVZ1-2A at Mohawk (unsaturated) & $\mathrm{R}$ & $08 / 24 / 04$ & 1015 & $<.02$ & $<.02$ & $<.02$ \\
\hline 395151085504530 & Vadose zone ACTVZ1-2A at Mohawk (unsaturated) & $\mathrm{R}$ & $10 / 20 / 04$ & 1300 & $<.02$ & $<.02$ & $<.02$ \\
\hline 395151085504530 & Vadose zone ACTVZ1-2A at Mohawk (saturated) & $\mathrm{R}$ & 06/22/05 & 0900 & $<.02$ & $<.02$ & $<.02$ \\
\hline 395151085504531 & Vadose zone ACTVZ1-2A at Mohawk (saturated) & $\mathrm{R}$ & $05 / 25 / 04$ & 1025 & .02 & .08 & $<.02$ \\
\hline 395151085504531 & Vadose zone ACTVZ1-2B at Mohawk (unsaturated) & $\mathrm{R}$ & $08 / 24 / 04$ & 1020 & $<.02$ & .11 & $<.02$ \\
\hline 395151085504531 & Vadose zone ACTVZ1-2B at Mohawk (unsaturated) & $\mathrm{R}$ & $10 / 20 / 04$ & 1310 & $<.02$ & $<.02$ & $<.02$ \\
\hline 395151085504531 & Vadose zone ACTVZ1-2B at Mohawk (unsaturated) & $\mathrm{R}$ & 06/22/05 & 0910 & $<.02$ & .12 & $<.02$ \\
\hline 395151085504532 & Vadose zone ACTVZ1-2C at Mohawk (unsaturated) & $\mathrm{R}$ & $08 / 24 / 04$ & 1025 & $<.02$ & .16 & $<.02$ \\
\hline 395151085504532 & Vadose zone ACTVZ1-2C at Mohawk (unsaturated) & $\mathrm{R}$ & $10 / 20 / 04$ & 1320 & .05 & $<.02$ & $<.02$ \\
\hline 395151085504532 & Vadose zone ACTVZ1-2C at Mohawk (saturated) & $\mathrm{R}$ & $06 / 22 / 05$ & 0920 & $<.02$ & .09 & $<.02$ \\
\hline 395151085504533 & Vadose zone ACTVZ1-2D at Mohawk (saturated) & $\mathrm{R}$ & $08 / 24 / 04$ & 1030 & $<.02$ & .12 & $<.02$ \\
\hline 395151085504533 & Vadose zone ACTVZ1-2D at Mohawk (saturated) & $\mathrm{R}$ & 06/22/05 & 0930 & $<.02$ & .08 & $<.02$ \\
\hline 395025085493501 & Well ACTAS1-3A at Mohawk & $\mathrm{R}$ & $06 / 21 / 04$ & 1245 & $<.02$ & .05 & $<.02$ \\
\hline 395025085493501 & Well ACTAS1-3A at Mohawk & FR & $06 / 21 / 04$ & 1246 & $<.02$ & .05 & $<.02$ \\
\hline 395025085493501 & Well ACTAS1-3A at Mohawk & FR & $06 / 21 / 04$ & 1248 & $<.02$ & .04 & $<.02$ \\
\hline 395025085493501 & Well ACTAS1-3A at Mohawk & $\mathrm{R}$ & $10 / 15 / 04$ & 1200 & $<.02$ & $<.02$ & $<.02$ \\
\hline 395046085510903 & Well ACTAS1-1B at Mohawk (saturated) & $\mathrm{R}$ & $06 / 22 / 04$ & 1430 & .13 & .16 & $<.02$ \\
\hline 395046085510903 & Well ACTAS1-1B at Mohawk (saturated) & $\mathrm{R}$ & $06 / 22 / 05$ & 1200 & .12 & $<.02$ & $<.02$ \\
\hline 395046085510901 & Well ACTAS1-1C at Mohawk (saturated) & $\mathrm{R}$ & $10 / 21 / 04$ & 1100 & $<.02$ & .10 & $<.02$ \\
\hline 395046085510901 & Well ACTAS1-1C at Mohawk (saturated) & $\mathrm{R}$ & $06 / 22 / 05$ & 1300 & .08 & $<.02$ & $<.02$ \\
\hline
\end{tabular}


Table 6. Analytical results for glyphosate, aminomethylphosphonic acid, and glufosinate in Toxic Substances Hydrology Program studies, 2001-06.Continued

[Sample type: FB, field blank; FR, field replicate; R, regular; LD, laboratory duplicate; --, no data; <, less than; $\mu \mathrm{g} / \mathrm{L}$, micrograms per liter]

\begin{tabular}{|c|c|c|c|c|c|c|c|}
\hline \multirow[b]{2}{*}{ Site number } & \multirow[b]{2}{*}{ Site location } & \multirow[b]{2}{*}{$\begin{array}{l}\text { Sam- } \\
\text { ple } \\
\text { type }\end{array}$} & \multirow[b]{2}{*}{$\begin{array}{c}\text { Date of } \\
\text { collection } \\
\text { (month/day/ } \\
\text { year) }\end{array}$} & \multirow[b]{2}{*}{$\begin{array}{l}\text { Col- } \\
\text { lec- } \\
\text { tion } \\
\text { time } \\
\text { (24- } \\
\text { hour) }\end{array}$} & \multicolumn{3}{|c|}{ Concentrations } \\
\hline & & & & & $\begin{array}{c}\text { Glypho- } \\
\text { sate } \\
\text { ( } \mu \mathrm{g} / \mathrm{L} \text { ) }\end{array}$ & $\begin{array}{l}\text { Amino- } \\
\text { meth- } \\
\text { ylphos- } \\
\text { phonic } \\
\text { acid } \\
\text { ( } \mathrm{gg} / \mathrm{L} \text { ) }\end{array}$ & $\begin{array}{c}\text { Glufos- } \\
\text { inate } \\
(\mu \mathrm{g} / \mathrm{L})\end{array}$ \\
\hline \multicolumn{8}{|c|}{ Sensitive environments study, 2004-06-Continued } \\
\hline \multicolumn{8}{|c|}{ Leary Weber Ditch Basin, Indiana (ground water) —Continued } \\
\hline 395046085510902 & Well ACTAS1-1D at Mohawk (saturated) & $\mathrm{R}$ & $10 / 21 / 04$ & 1200 & 0.03 & $<0.02$ & $<0.02$ \\
\hline 395046085510902 & Well ACTAS1-1D at Mohawk & $\mathrm{R}$ & $06 / 22 / 04$ & 1530 & $<.02$ & .05 & $<.02$ \\
\hline 395046085510902 & Well ACTAS1-1D at Mohawk & $\mathrm{R}$ & $06 / 22 / 05$ & 1230 & $<.02$ & $<.02$ & $<.02$ \\
\hline 395151085504504 & Well ACTAS1-2A at Mohawk & $\mathrm{R}$ & $06 / 22 / 04$ & 1100 & .04 & .09 & $<.02$ \\
\hline 395151085504504 & Well ACTAS1-2A at Mohawk & $\mathrm{R}$ & $06 / 22 / 05$ & 0930 & .02 & .11 & $<.02$ \\
\hline 395151085504503 & Well ACTAS1-2B at Mohawk & $\mathrm{R}$ & $11 / 08 / 04$ & 1130 & $<.02$ & .17 & $<.02$ \\
\hline 395151085504503 & Well ACTAS1-2B at Mohawk & $\mathrm{R}$ & 06/22/05 & 1000 & $<.02$ & .06 & $<.02$ \\
\hline 395151085504502 & Well ACTAS1-2C at Mohawk & $\mathrm{R}$ & 06/22/05 & 1030 & .04 & .05 & $<.02$ \\
\hline 395151085504501 & Well ACTAS1-2D at Mohawk & $\mathrm{R}$ & $06 / 22 / 04$ & 1200 & $<.02$ & .05 & $<.02$ \\
\hline 395151085504501 & Well ACTAS1-2D at Mohawk & $\mathrm{R}$ & $11 / 08 / 04$ & 1200 & $<.02$ & $<.02$ & $<.02$ \\
\hline 395151085504501 & Well ACTAS1-2D at Mohawk & $\mathrm{R}$ & 06/22/05 & 1100 & $<.02$ & $<.02$ & $<.02$ \\
\hline \multicolumn{8}{|c|}{ Leary Weber Ditch Basin, Indiana (surface water) } \\
\hline 03361638 & Leary Weber Ditch near Mohawk (composite) & $\mathrm{R}$ & $11 / 02 / 04$ & 0845 & $<.02$ & $<.02$ & $<.02$ \\
\hline 03361638 & Leary Weber Ditch near Mohawk & $\mathrm{R}$ & $11 / 02 / 04$ & 0845 & 1.7 & .13 & $<.02$ \\
\hline 03361638 & Leary Weber Ditch near Mohawk & $\mathrm{R}$ & $11 / 02 / 04$ & 1045 & .14 & .26 & $<.02$ \\
\hline 03361638 & Leary Weber Ditch near Mohawk & $\mathrm{R}$ & $11 / 02 / 04$ & 1345 & $<.02$ & $<.02$ & $<.02$ \\
\hline 03361638 & Leary Weber Ditch near Mohawk & $\mathrm{R}$ & $11 / 03 / 04$ & 1100 & $<.02$ & $<.02$ & $<.02$ \\
\hline 03361638 & Leary Weber Ditch near Mohawk & $\mathrm{R}$ & $04 / 19 / 05$ & 1845 & $<.02$ & $<.02$ & $<.02$ \\
\hline 03361638 & Leary Weber Ditch near Mohawk & $\mathrm{R}$ & $03 / 22 / 04$ & 1015 & $<.02$ & $<.02$ & $<.02$ \\
\hline 03361638 & Leary Weber Ditch near Mohawk & $\mathrm{R}$ & 03/30/04 & 1015 & $<.02$ & .10 & $<.02$ \\
\hline 03361638 & Leary Weber Ditch near Mohawk & LD & $03 / 30 / 04$ & 1015 & $<.02$ & .06 & $<.02$ \\
\hline 03361638 & Leary Weber Ditch near Mohawk & $\mathrm{R}$ & $05 / 14 / 04$ & 1545 & .50 & .06 & $<.02$ \\
\hline 03361638 & Leary Weber Ditch near Mohawk & $\mathrm{R}$ & $05 / 19 / 04$ & 0315 & 2.1 & .23 & $<.02$ \\
\hline 03361638 & Leary Weber Ditch near Mohawk & FR & $05 / 19 / 04$ & 0315 & 1.1 & .23 & $<.02$ \\
\hline 03361638 & Leary Weber Ditch near Mohawk & $\mathrm{R}$ & 05/19/04 & 0715 & 1.1 & .13 & $<.02$ \\
\hline 03361638 & Leary Weber Ditch near Mohawk & $\mathrm{R}$ & $05 / 19 / 04$ & 1100 & .72 & .23 & $<.02$ \\
\hline 03361638 & Leary Weber Ditch near Mohawk & $\mathrm{R}$ & 05/20/04 & 0330 & .27 & .08 & $<.02$ \\
\hline
\end{tabular}


Table 6. Analytical results for glyphosate, aminomethylphosphonic acid, and glufosinate in Toxic Substances Hydrology Program studies, 2001-06. Continued

[Sample type: FB, field blank; FR, field replicate; R, regular; LD, laboratory duplicate; --, no data; <, less than; $\mu \mathrm{g} / \mathrm{L}$, micrograms per liter]

\begin{tabular}{|c|c|c|c|c|c|c|c|}
\hline \multirow[b]{2}{*}{ Site number } & \multirow[b]{2}{*}{ Site location } & \multirow[b]{2}{*}{$\begin{array}{l}\text { Sam- } \\
\text { ple } \\
\text { type }\end{array}$} & \multirow[b]{2}{*}{$\begin{array}{c}\text { Date of } \\
\text { collection } \\
\text { (month/day/ } \\
\text { year) }\end{array}$} & \multirow[b]{2}{*}{$\begin{array}{l}\text { Col- } \\
\text { lec- } \\
\text { tion } \\
\text { time } \\
\text { (24- } \\
\text { hour) }\end{array}$} & \multicolumn{3}{|c|}{ Concentrations } \\
\hline & & & & & $\begin{array}{c}\text { Glypho- } \\
\text { sate } \\
\text { ( } \mu \mathrm{g} / \mathrm{L})\end{array}$ & $\begin{array}{c}\text { Amino- } \\
\text { meth- } \\
\text { ylphos- } \\
\text { phonic } \\
\text { acid } \\
\text { ( } \mu \mathrm{g} / \mathrm{L})\end{array}$ & $\begin{array}{c}\text { Glufos- } \\
\text { inate } \\
\text { ( } \mu \mathrm{g} / \mathrm{L})\end{array}$ \\
\hline \multicolumn{8}{|c|}{ Sensitive environments study, 2004-06-Continued } \\
\hline \multicolumn{8}{|c|}{ Leary Weber Ditch Basin, Indiana (surface water) - Continued } \\
\hline 03361638 & Leary Weber Ditch near Mohawk & $\mathrm{R}$ & $05 / 20 / 04$ & 1600 & 0.16 & 0.14 & $<0.02$ \\
\hline 03361638 & Leary Weber Ditch near Mohawk & $\mathrm{R}$ & $05 / 30 / 04$ & 2200 & .47 & .18 & $<.02$ \\
\hline 03361638 & Leary Weber Ditch near Mohawk & $\mathrm{R}$ & 05/30/04 & 2200 & 2.1 & .62 & $<.02$ \\
\hline 03361638 & Leary Weber Ditch near Mohawk & $\mathrm{R}$ & $05 / 31 / 04$ & 0100 & 5.5 & .58 & $<.02$ \\
\hline 03361638 & Leary Weber Ditch near Mohawk & $\mathrm{R}$ & $05 / 31 / 04$ & 0400 & 4.9 & .39 & $<.02$ \\
\hline 03361638 & Leary Weber Ditch near Mohawk & $\mathrm{R}$ & $05 / 31 / 04$ & 2045 & 1.1 & .07 & $<.02$ \\
\hline 03361638 & Leary Weber Ditch near Mohawk & $\mathrm{R}$ & 06/01/04 & 1400 & .55 & .25 & $<.02$ \\
\hline 03361638 & Leary Weber Ditch near Mohawk & $\mathrm{R}$ & 06/01/04 & 1401 & .54 & .31 & $<.02$ \\
\hline 03361638 & Leary Weber Ditch near Mohawk & $\mathrm{R}$ & 06/30/04 & 1800 & .08 & .23 & $<.02$ \\
\hline 03361638 & Leary Weber Ditch near Mohawk & FB & $08 / 26 / 04$ & 1000 & $<.02$ & $<.02$ & $<.02$ \\
\hline 03361638 & Leary Weber Ditch near Mohawk & $\mathrm{R}$ & 06/12/05 & 2045 & .20 & $<.02$ & $<.02$ \\
\hline 03361638 & Leary Weber Ditch near Mohawk & $\mathrm{R}$ & $06 / 12 / 05$ & 2245 & .49 & .56 & $<.02$ \\
\hline 03361638 & Leary Weber Ditch near Mohawk & $\mathrm{R}$ & $06 / 13 / 05$ & 0045 & 2.0 & .81 & 1.5 \\
\hline 03361638 & Leary Weber Ditch near Mohawk & $\mathrm{R}$ & 06/13/05 & 0145 & 2.9 & .69 & .24 \\
\hline 03361638 & Leary Weber Ditch near Mohawk & $\mathrm{R}$ & 06/13/05 & 1345 & .52 & .33 & $<.02$ \\
\hline 03361638 & Leary Weber Ditch near Mohawk & $\mathrm{R}$ & $06 / 14 / 05$ & 1215 & .15 & .19 & $<.02$ \\
\hline 395120085502401 & Overland flow ACTOF1-1 at Mohawk & $\mathrm{R}$ & 05/19/04 & 0128 & 310 & 27 & $<0.02$ \\
\hline 395120085502401 & Overland flow ACTOF1-1 at Mohawk & $\mathrm{R}$ & 05/19/04 & 0158 & 418 & 25 & $<.02$ \\
\hline 395120085502401 & Overland flow ACTOF1-1 at Mohawk & $\mathrm{R}$ & 05/19/04 & 0228 & 427 & 29 & $<.02$ \\
\hline 395120085502401 & Overland flow ACTOF1-1 at Mohawk & $\mathrm{R}$ & 05/19/04 & 0258 & 376 & 24 & $<.02$ \\
\hline 395120085502401 & Overland flow ACTOF1-1 at Mohawk & $\mathrm{R}$ & $05 / 19 / 04$ & 0410 & 359 & 26 & $<.02$ \\
\hline 395120085502401 & Overland flow ACTOF1-1 at Mohawk & FR & $05 / 19 / 04$ & 0411 & 385 & 25 & $<.02$ \\
\hline 395120085502401 & Overland flow ACTOF1-1 at Mohawk & $\mathrm{R}$ & $05 / 19 / 04$ & 0500 & 377 & 28 & $<.02$ \\
\hline 395120085502401 & Overland flow ACTOF1-1 at Mohawk & $\mathrm{R}$ & $05 / 30 / 04$ & 2130 & 41 & 8.2 & $<.02$ \\
\hline 395120085502401 & Overland flow ACTOF1-1 at Mohawk & $\mathrm{R}$ & 05/30/04 & 2230 & 22 & 3.7 & $<.02$ \\
\hline 395120085502401 & Overland flow ACTOF1-1 at Mohawk & $\mathrm{R}$ & 05/30/04 & 2330 & 25 & 4.3 & $<.02$ \\
\hline 395120085502401 & Overland flow ACTOF1-1 at Mohawk & $\mathrm{R}$ & $05 / 31 / 04$ & 0201 & 31 & 6.7 & $<.02$ \\
\hline 395120085502401 & Overland flow ACTOF1-1 at Mohawk & FR & $05 / 31 / 04$ & 0200 & 38 & 8.5 & $<.02$ \\
\hline
\end{tabular}


Table 6. Analytical results for glyphosate, aminomethylphosphonic acid, and glufosinate in Toxic Substances Hydrology Program studies, 2001-06. Continued

[Sample type: FB, field blank; FR, field replicate; R, regular; LD, laboratory duplicate; --, no data; <, less than; $\mu$ g/L, micrograms per liter]

\begin{tabular}{|c|c|c|c|c|c|c|c|}
\hline \multirow[b]{2}{*}{ Site number } & \multirow[b]{2}{*}{ Site location } & \multirow[b]{2}{*}{$\begin{array}{c}\text { Sam- } \\
\text { ple } \\
\text { type }\end{array}$} & \multirow[b]{2}{*}{$\begin{array}{c}\text { Date of } \\
\text { collection } \\
\text { (month/day/ } \\
\text { year) }\end{array}$} & \multirow[b]{2}{*}{$\begin{array}{c}\text { Col- } \\
\text { lec- } \\
\text { tion } \\
\text { time } \\
\text { (24- } \\
\text { hour) }\end{array}$} & \multicolumn{3}{|c|}{ Concentrations } \\
\hline & & & & & $\begin{array}{c}\text { Glypho- } \\
\text { sate } \\
\text { ( } \mu \mathrm{g} / \mathrm{L} \text { ) }\end{array}$ & $\begin{array}{l}\text { Amino- } \\
\text { meth- } \\
\text { ylphos- } \\
\text { phonic } \\
\text { acid } \\
\text { ( } \mu \mathrm{g} / \mathrm{L})\end{array}$ & $\begin{array}{c}\text { Glufos- } \\
\text { inate } \\
(\mu \mathrm{g} / \mathrm{L})\end{array}$ \\
\hline \multicolumn{8}{|c|}{ Sensitive environments study, 2004-06-Continued } \\
\hline \multicolumn{8}{|c|}{ Leary Weber Ditch Basin, Indiana (surface water)_Continued } \\
\hline 395120085502401 & Overland flow ACTOF1-1 at Mohawk & $\mathrm{R}$ & $05 / 31 / 04$ & 0530 & 49 & 11 & $<0.02$ \\
\hline 395120085502401 & Overland flow ACTOF1-1 at Mohawk & $\mathrm{R}$ & $05 / 31 / 04$ & 1050 & 34 & 16 & $<.02$ \\
\hline 395120085502401 & Overland flow ACTOF1-1 at Mohawk & FB & $08 / 26 / 04$ & 1100 & $<.02$ & $<.02$ & $<.02$ \\
\hline 395120085502401 & Overland flow ACTOF1-1 at Mohawk & $\mathrm{R}$ & $06 / 12 / 05$ & 2100 & .20 & 1.3 & $<.02$ \\
\hline 394340085524601 & Sugar Creek at Co Road 400 South at New Palestine & $\mathrm{R}$ & $05 / 19 / 04$ & 0200 & .32 & .21 & $<.02$ \\
\hline 394340085524601 & Sugar Creek at Co Road 400 South at New Palestine & $\mathrm{R}$ & $05 / 20 / 04$ & 1200 & .20 & .12 & $<.02$ \\
\hline 394340085524601 & Sugar Creek at Co Road 400 South at New Palestine & $\mathrm{R}$ & $05 / 21 / 04$ & 1330 & .15 & .14 & $<.02$ \\
\hline 394340085524601 & Sugar Creek at Co Road 400 South at New Palestine & $\mathrm{R}$ & $05 / 14 / 04$ & 2100 & .06 & .09 & $<.02$ \\
\hline 394340085524601 & Sugar Creek at Co Road 400 South at New Palestine & $\mathrm{R}$ & $05 / 19 / 04$ & 0600 & .66 & .07 & $<.02$ \\
\hline 394340085524601 & Sugar Creek at Co Road 400 South at New Palestine & $\mathrm{R}$ & $05 / 19 / 04$ & 1000 & 1.0 & .36 & $<.02$ \\
\hline 394340085524601 & Sugar Creek at Co Road 400 South at New Palestine & $\mathrm{R}$ & $05 / 31 / 04$ & 0430 & 1.0 & .19 & $<.02$ \\
\hline 394340085524601 & Sugar Creek at Co Road 400 South at New Palestine & $\mathrm{R}$ & $11 / 02 / 04$ & 0400 & .03 & .07 & $<.02$ \\
\hline 394340085524601 & Sugar Creek at Co Road 400 South at New Palestine & $\mathrm{R}$ & $11 / 02 / 04$ & 0400 & .04 & $<.02$ & $<.02$ \\
\hline 394340085524601 & Sugar Creek at Co Road 400 South at New Palestine & $\mathrm{R}$ & $11 / 02 / 04$ & 1000 & .04 & $<.02$ & $<.02$ \\
\hline 394340085524601 & Sugar Creek at Co Road 400 South at New Palestine & $\mathrm{R}$ & $11 / 03 / 04$ & 1200 & $<.02$ & $<.02$ & $<.02$ \\
\hline 394340085524601 & Sugar Creek at Co Road 400 South at New Palestine & $\mathrm{R}$ & $05 / 31 / 04$ & 1030 & 1.6 & .23 & $<.02$ \\
\hline 394340085524601 & Sugar Creek at Co Road 400 South at New Palestine & $\mathrm{R}$ & $05 / 31 / 04$ & 0430 & .74 & .39 & $<.02$ \\
\hline 394340085524601 & Sugar Creek at Co Road 400 South at New Palestine & $\mathrm{R}$ & $06 / 01 / 04$ & 1015 & 66 & .48 & $<.02$ \\
\hline 394340085524601 & Sugar Creek at Co Road 400 South at New Palestine & $\mathrm{R}$ & $06 / 02 / 04$ & 1630 & .51 & .74 & $<.02$ \\
\hline 394340085524601 & Sugar Creek at Co Road 400 South at New Palestine & $\mathrm{R}$ & $06 / 02 / 04$ & 1631 & .37 & .44 & $<.02$ \\
\hline 394340085524601 & Sugar Creek at Co Road 400 South at New Palestine & FB & $08 / 26 / 04$ & 0900 & $<.02$ & $<.02$ & $<.02$ \\
\hline 394340085524601 & Sugar Creek at Co Road 400 South at New Palestine & $\mathrm{R}$ & $11 / 02 / 04$ & 1200 & .05 & .17 & $<.02$ \\
\hline \multicolumn{8}{|c|}{ Leary Weber Ditch Basin, Indiana (rainfall) } \\
\hline 395151085504595 & Rain sampler ACTRS1-2 at Mohawk & $\mathrm{R}$ & $04 / 27 / 04$ & 1100 & 1.1 & .07 & $<.02$ \\
\hline 395151085504595 & Rain sampler ACTRS1-2 at Mohawk & $\mathrm{R}$ & $05 / 12 / 04$ & 0930 & .67 & .21 & $<.02$ \\
\hline 395151085504595 & Rain sampler ACTRS1-2 at Mohawk & $\mathrm{R}$ & $05 / 18 / 04$ & 1015 & .15 & .06 & $<.02$ \\
\hline 395151085504595 & Rain sampler ACTRS1-2 at Mohawk & $\mathrm{R}$ & $05 / 25 / 04$ & 0930 & .04 & .06 & $<.02$ \\
\hline 395151085504595 & Rain sampler ACTRS1-2 at Mohawk & $\mathrm{R}$ & $06 / 08 / 04$ & 1000 & .12 & .08 & $<.02$ \\
\hline
\end{tabular}


Table 6. Analytical results for glyphosate, aminomethylphosphonic acid, and glufosinate in Toxic Substances Hydrology Program studies, 2001-06. Continued

[Sample type: FB, field blank; FR, field replicate; R, regular; LD, laboratory duplicate; --, no data; <, less than; $\mu \mathrm{g} / \mathrm{L}$, micrograms per liter]

\begin{tabular}{|c|c|c|c|c|c|c|c|}
\hline \multirow[b]{2}{*}{ Site number } & \multirow[b]{2}{*}{ Site location } & \multirow[b]{2}{*}{$\begin{array}{c}\text { Sam- } \\
\text { ple } \\
\text { type }\end{array}$} & \multirow[b]{2}{*}{$\begin{array}{c}\text { Date of } \\
\text { collection } \\
\text { (month/day/ } \\
\text { year) }\end{array}$} & \multirow[b]{2}{*}{$\begin{array}{l}\text { Col- } \\
\text { lec- } \\
\text { tion } \\
\text { time } \\
\text { (24- } \\
\text { hour) }\end{array}$} & \multicolumn{3}{|c|}{ Concentrations } \\
\hline & & & & & $\begin{array}{c}\text { Glypho- } \\
\text { sate } \\
\text { ( } \mu \mathrm{g} / \mathrm{L})\end{array}$ & 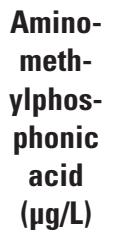 & $\begin{array}{c}\text { Glufos- } \\
\text { inate } \\
(\mu \mathrm{g} / \mathrm{L})\end{array}$ \\
\hline \multicolumn{8}{|c|}{ Sensitive environments study, 2004-06-Continued } \\
\hline \multicolumn{8}{|c|}{ Leary Weber Ditch Basin, Indiana (rainfall)_Continued } \\
\hline 395151085504595 & Rain sampler ACTRS1-2 at Mohawk (composite) & $\mathrm{R}$ & $06 / 15 / 04$ & 1000 & 0.10 & 0.08 & $<0.02$ \\
\hline 395151085504595 & Rain sampler ACTRS1-2 at Mohawk (composite) & $\mathrm{R}$ & $06 / 29 / 04$ & 1030 & .24 & .17 & $<.02$ \\
\hline 395151085504595 & Rain sampler ACTRS1-2 at Mohawk (composite) & $\mathrm{R}$ & $07 / 06 / 04$ & 1215 & .20 & .07 & $<.02$ \\
\hline 395151085504595 & Rain sampler ACTRS1-2 at Mohawk (composite) & $\mathrm{R}$ & $07 / 20 / 04$ & 1200 & .20 & .47 & $<.02$ \\
\hline 395151085504595 & Rain sampler ACTRS1-2 at Mohawk (composite) & $\mathrm{R}$ & 08/03/04 & 1100 & .06 & .02 & $<.02$ \\
\hline 395151085504595 & Rain sampler ACTRS1-2 at Mohawk (composite) & $\mathrm{R}$ & $08 / 17 / 04$ & 1200 & .11 & .04 & $<.02$ \\
\hline 395151085504595 & Rain sampler ACTRS1-2 at Mohawk (composite) & $\mathrm{R}$ & $08 / 24 / 04$ & 0945 & $<.02$ & .03 & $<.02$ \\
\hline 395151085504595 & Rain sampler ACTRS1-2 at Mohawk & $\mathrm{R}$ & $11 / 08 / 04$ & 1000 & $<.02$ & $<.02$ & $<.02$ \\
\hline 395151085504595 & Rain sampler ACTRS1-2 at Mohawk & $\mathrm{R}$ & 06/07/05 & 1045 & .03 & $<.02$ & $<.02$ \\
\hline
\end{tabular}


Table 6. Analytical results for glyphosate, aminomethylphosphonic acid, and glufosinate in Toxic Substances Hydrology Program studies, 2001-06. Continued

[Sample type: FB, field blank; FR, field replicate; R, regular; LD, laboratory duplicate; --, no data; <, less than; $\mu \mathrm{g} / \mathrm{L}$, micrograms per liter]

\begin{tabular}{|c|c|c|c|c|c|c|c|}
\hline \multirow[b]{2}{*}{ Site number } & \multirow[b]{2}{*}{ Site location/soil core depth in inches (“) } & \multirow[b]{2}{*}{$\begin{array}{c}\text { Sam- } \\
\text { ple } \\
\text { type }\end{array}$} & \multirow[b]{2}{*}{$\begin{array}{c}\text { Date of } \\
\text { collection } \\
\text { (month/day/ } \\
\text { year) }\end{array}$} & \multirow[b]{2}{*}{$\begin{array}{l}\text { Col- } \\
\text { lec- } \\
\text { tion } \\
\text { time } \\
\text { (24- } \\
\text { hour) }\end{array}$} & \multicolumn{3}{|c|}{ Concentrations } \\
\hline & & & & & $\begin{array}{c}\text { Glypho- } \\
\text { sate } \\
\text { ( } \mu \mathrm{g} / \mathrm{L})\end{array}$ & $\begin{array}{l}\text { Amino- } \\
\text { meth- } \\
\text { ylphos- } \\
\text { phonic } \\
\text { acid } \\
(\mu \mathrm{g} / \mathrm{L})\end{array}$ & $\begin{array}{l}\text { Glufos- } \\
\text { inate } \\
(\mu \mathrm{g} / \mathrm{L})\end{array}$ \\
\hline \multicolumn{8}{|c|}{ Sensitive environments study, 2004-06-Continued } \\
\hline \multicolumn{8}{|c|}{ Leary Weber Ditch Basin, Indiana (soils) } \\
\hline 395045085510930 & Vadose zone ACTVZ1-1A at Mohawk (South, 0-6") & $\mathrm{R}$ & $04 / 15 / 04$ & 1000 & 23 & 109 & -- \\
\hline 395045085510930 & Vadose zone ACTVZ1-1A at Mohawk (South, 0-6") & $\mathrm{R}$ & $04 / 15 / 04$ & 1001 & 12 & 109 & -- \\
\hline 395045085510930 & Vadose zone ACTVZ1-1A at Mohawk (South, 0-6") & $\mathrm{R}$ & $04 / 15 / 04$ & 1002 & 7.0 & 70 & -- \\
\hline 395045085510930 & Vadose zone ACTVZ1-1A at Mohawk (South, 0-6") & $\mathrm{R}$ & $05 / 24 / 04$ & 1500 & 110 & 112 & -- \\
\hline 395045085510930 & Vadose zone ACTVZ1-1A at Mohawk (South, 0-6") & $\mathrm{R}$ & 05/24/04 & 1501 & 332 & 91 & -- \\
\hline 395045085510930 & Vadose zone ACTVZ1-1A at Mohawk (South, 0-6") & $\mathrm{LD}$ & $05 / 24 / 04$ & 1501 & 269 & 99 & -- \\
\hline 395045085510930 & Vadose zone ACTVZ1-1A at Mohawk (South, 0-6") & $\mathrm{R}$ & 05/24/04 & 1502 & 62 & 136 & -- \\
\hline 395045085510930 & Vadose zone ACTVZ1-1A at Mohawk (South, 6-12”) & $\mathrm{R}$ & 05/24/04 & 1700 & 9.0 & 14 & -- \\
\hline 395045085510930 & Vadose zone ACTVZ1-1A at Mohawk (South, 6-12”) & $\mathrm{R}$ & $05 / 24 / 04$ & 1701 & 14 & 9.0 & -- \\
\hline 395045085510930 & Vadose zone ACTVZ1-1A at Mohawk (South, 6-12”) & $\mathrm{R}$ & 05/24/04 & 1702 & 12 & 22 & -- \\
\hline 395045085510930 & Vadose zone ACTVZ1-1A at Mohawk (South, 12-18") & $\mathrm{R}$ & $05 / 24 / 04$ & 1730 & 11 & 7.0 & -- \\
\hline 395045085510930 & Vadose zone ACTVZ1-1A at Mohawk (South, 12-18”) & $\mathrm{R}$ & $05 / 24 / 04$ & 1731 & 10 & 10 & -- \\
\hline 395045085510930 & Vadose zone ACTVZ1-1A at Mohawk (South, 12-18”) & $\mathrm{R}$ & 05/24/04 & 1732 & 12 & 13 & -- \\
\hline 395045085510930 & Vadose zone ACTVZ1-1A at Mohawk (South, 0-6”) & $\mathrm{R}$ & 07/07/04 & 1500 & 55 & 167 & -- \\
\hline 395045085510930 & Vadose zone ACTVZ1-1A at Mohawk (South, 0-6") & $\mathrm{R}$ & 07/07/04 & 1501 & 163 & 185 & -- \\
\hline 395045085510930 & Vadose zone ACTVZ1-1A at Mohawk (South, 0-6") & $\mathrm{R}$ & 07/07/04 & 1502 & 33 & 116 & -- \\
\hline 395045085510930 & Vadose zone ACTVZ1-1A at Mohawk (South, 6-12”) & $\mathrm{R}$ & 07/07/04 & 1530 & 8.6 & 21 & -- \\
\hline 395045085510930 & Vadose zone ACTVZ1-1A at Mohawk (South, 6-12”) & $\mathrm{R}$ & 07/07/04 & 1531 & 22 & 23 & -- \\
\hline 395045085510930 & Vadose zone ACTVZ1-1A at Mohawk (South, 6-12”) & $\mathrm{R}$ & 07/07/04 & 1532 & 46 & 25 & -- \\
\hline 395045085510930 & Vadose zone ACTVZ1-1A at Mohawk (South, 12-18”) & $\mathrm{R}$ & 07/07/04 & 1600 & 11 & 12 & -- \\
\hline 395045085510930 & Vadose zone ACTVZ1-1A at Mohawk (South, 12-18") & $\mathrm{R}$ & 07/07/04 & 1601 & 15 & 18 & -- \\
\hline 395045085510930 & Vadose zone ACTVZ1-1A at Mohawk (South, 12-18”) & $\mathrm{R}$ & 07/07/04 & 1602 & 4.9 & 9.2 & -- \\
\hline 395045085510930 & Vadose zone ACTVZ1-1A at Mohawk (South, 0-6") & $\mathrm{R}$ & $10 / 21 / 04$ & 1200 & 27 & 132 & $<1.0$ \\
\hline 395045085510930 & Vadose zone ACTVZ1-1A at Mohawk (South, 0-6”) & $\mathrm{R}$ & $10 / 21 / 04$ & 1201 & 6.9 & 69 & $<1.0$ \\
\hline 395045085510930 & Vadose zone ACTVZ1-1A at Mohawk (South, 0-6”) & $\mathrm{R}$ & $10 / 21 / 04$ & 1202 & 44 & 105 & $<1.0$ \\
\hline 395045085510930 & Vadose zone ACTVZ1-1A at Mohawk (South, 0-6”) & LD & $10 / 21 / 04$ & 1202 & 46 & 113 & $<1.0$ \\
\hline 395045085510930 & Vadose zone ACTVZ1-1A at Mohawk (South, 6-12”) & $\mathrm{R}$ & $10 / 21 / 04$ & 1210 & $<1.0$ & 2.2 & $<1.0$ \\
\hline 395045085510930 & Vadose zone ACTVZ1-1A at Mohawk (South, 6-12”) & $\mathrm{R}$ & $10 / 21 / 04$ & 1211 & $<1.0$ & $<1.0$ & $<1.0$ \\
\hline
\end{tabular}


Table 6. Analytical results for glyphosate, aminomethylphosphonic acid, and glufosinate in Toxic Substances Hydrology Program studies, 2001-06. Continued

[Sample type: FB, field blank; FR, field replicate; R, regular; LD, laboratory duplicate; --, no data; <, less than; $\mu \mathrm{g} / \mathrm{L}$, micrograms per liter]

\begin{tabular}{|c|c|c|c|c|c|c|c|}
\hline \multirow[b]{2}{*}{ Site number } & \multirow[b]{2}{*}{ Site location/soil core depth in inches (“) } & \multirow[b]{2}{*}{$\begin{array}{l}\text { Sam- } \\
\text { ple } \\
\text { type }\end{array}$} & \multirow[b]{2}{*}{$\begin{array}{c}\text { Date of } \\
\text { collection } \\
\text { (month/day/ } \\
\text { year) }\end{array}$} & \multirow[b]{2}{*}{$\begin{array}{c}\text { Col- } \\
\text { lec- } \\
\text { tion } \\
\text { time } \\
\text { (24- } \\
\text { hour) }\end{array}$} & \multicolumn{3}{|c|}{ Concentrations } \\
\hline & & & & & $\begin{array}{c}\text { Glypho- } \\
\text { sate } \\
\text { ( } \mu \mathrm{g} / \mathrm{L})\end{array}$ & 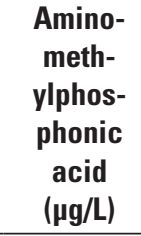 & $\begin{array}{c}\text { Glufos- } \\
\text { inate } \\
\text { ( } \mu \mathrm{g} / \mathrm{L})\end{array}$ \\
\hline \multicolumn{8}{|c|}{ Sensitive environments study, 2004-06-Continued } \\
\hline \multicolumn{8}{|c|}{ Leary Weber Ditch Basin, Indiana (soils)_Continued } \\
\hline 395045085510930 & Vadose zone ACTVZ1-1A at Mohawk (South, 6-12") & $\mathrm{R}$ & $10 / 21 / 04$ & 1212 & $<1.0$ & 3.8 & $<1.0$ \\
\hline 395045085510930 & Vadose zone ACTVZ1-1A at Mohawk (South, 12-18”) & $\mathrm{R}$ & $10 / 21 / 04$ & 1220 & $<1.0$ & $<1.0$ & $<1.0$ \\
\hline 395045085510930 & Vadose zone ACTVZ1-1A at Mohawk (South, 12-18”) & $\mathrm{R}$ & $10 / 21 / 04$ & 1221 & $<1.0$ & $<1.0$ & $<1.0$ \\
\hline 395045085510930 & Vadose zone ACTVZ1-1A at Mohawk (South, 12-18”) & $\mathrm{R}$ & $10 / 21 / 04$ & 1222 & $<1.0$ & $<1.0$ & $<1.0$ \\
\hline 395045085510930 & Vadose zone ACTVZ1-1A at Mohawk (South, 0-6") & $\mathrm{R}$ & $04 / 19 / 05$ & 0930 & 7.2 & 39 & $<1.0$ \\
\hline 395045085510930 & Vadose zone ACTVZ1-1A at Mohawk (South, 0-6") & $\mathrm{R}$ & $04 / 19 / 05$ & 0940 & 2.2 & 43 & $<1.0$ \\
\hline 395045085510930 & Vadose zone ACTVZ1-1A at Mohawk (South, 0-6") & $\mathrm{R}$ & $04 / 19 / 05$ & 0941 & $<1.0$ & 10 & $<1.0$ \\
\hline 395045085510930 & Vadose zone ACTVZ1-1A at Mohawk (South, 6-12”) & $\mathrm{R}$ & $04 / 19 / 05$ & 0931 & 1.2 & 8.5 & $<1.0$ \\
\hline 395045085510930 & Vadose zone ACTVZ1-1A at Mohawk (South, 6-12") & $\mathrm{R}$ & $04 / 19 / 05$ & 0942 & 1.7 & 9.3 & $<1.0$ \\
\hline 395045085510930 & Vadose zone ACTVZ1-1A at Mohawk (South, 6-12”) & LD & $04 / 19 / 05$ & 0942 & 1.5 & 8.8 & $<1.0$ \\
\hline 395045085510930 & Vadose zone ACTVZ1-1A at Mohawk (South, 6-12”) & $\mathrm{R}$ & $04 / 19 / 05$ & 0950 & 6.4 & 47 & $<1.0$ \\
\hline 395045085510930 & Vadose zone ACTVZ1-1A at Mohawk (South, 12-18”) & $\mathrm{R}$ & $04 / 19 / 05$ & 0932 & $<1.0$ & 1.6 & $<1.0$ \\
\hline 395045085510930 & Vadose zone ACTVZ1-1A at Mohawk (South, 12-18”) & $\mathrm{R}$ & $04 / 19 / 05$ & 0951 & 1.8 & 9.8 & $<1.0$ \\
\hline 395045085510930 & Vadose zone ACTVZ1-1A at Mohawk (South, 12-18”) & $\mathrm{R}$ & $04 / 19 / 05$ & 0952 & $<1.0$ & 2.6 & $<1.0$ \\
\hline 395045085510930 & Vadose zone ACTVZ1-1A at Mohawk (South, 0-6”) & $\mathrm{R}$ & $05 / 25 / 05$ & 1500 & 12 & 68 & $<1.0$ \\
\hline 395045085510930 & Vadose zone ACTVZ1-1A at Mohawk (South, 0-6") & $\mathrm{R}$ & $05 / 25 / 05$ & 1510 & 6.0 & 33 & $<1.0$ \\
\hline 395045085510930 & Vadose zone ACTVZ1-1A at Mohawk (South, 0-6”) & $\mathrm{R}$ & $05 / 25 / 05$ & 1520 & 7.9 & 52 & $<1.0$ \\
\hline 395045085510930 & Vadose zone ACTVZ1-1A at Mohawk (South, 6-12”) & $\mathrm{R}$ & $05 / 25 / 05$ & 1501 & $<1.0$ & 2.5 & $<1.0$ \\
\hline 395045085510930 & Vadose zone ACTVZ1-1A at Mohawk (South, 6-12”) & $\mathrm{R}$ & $05 / 25 / 05$ & 1511 & $<1.0$ & 5.4 & $<1.0$ \\
\hline 395045085510930 & Vadose zone ACTVZ1-1A at Mohawk (South, 6-12”) & $\mathrm{R}$ & $05 / 25 / 05$ & 1521 & $<1.0$ & 4.9 & $<1.0$ \\
\hline 395045085510930 & Vadose zone ACTVZ1-1A at Mohawk (South, 12-18”) & $\mathrm{R}$ & $05 / 25 / 05$ & 1502 & $<1.0$ & $<1.0$ & $<1.0$ \\
\hline 395045085510930 & Vadose zone ACTVZ1-1A at Mohawk (South, 12-18”) & $\mathrm{R}$ & $05 / 25 / 05$ & 1512 & $<1.0$ & 2.0 & $<1.0$ \\
\hline 395045085510930 & Vadose zone ACTVZ1-1A at Mohawk (South, 12-18”) & $\mathrm{R}$ & $05 / 25 / 05$ & 1522 & $<1.0$ & $<1.0$ & $<1.0$ \\
\hline 395151085504530 & Vadose zone ACTVZ1-2A at Mohawk (North, 0-6”) & $\mathrm{R}$ & $04 / 15 / 04$ & 0900 & 7.0 & 144 & -- \\
\hline 395151085504530 & Vadose zone ACTVZ1-2A at Mohawk (North, 0-6") & LD & $04 / 15 / 04$ & 0900 & 8.0 & 144 & -- \\
\hline 395151085504530 & Vadose zone ACTVZ1-2A at Mohawk (North, 0-6”) & $\mathrm{R}$ & $04 / 15 / 04$ & 0901 & 6.0 & 112 & -- \\
\hline 395151085504530 & Vadose zone ACTVZ1-2A at Mohawk (North, 0-6") & $\mathrm{R}$ & $04 / 15 / 04$ & 0902 & 10 & 157 & -- \\
\hline 395151085504530 & Vadose zone ACTVZ1-2A at Mohawk (North, 0-6") & $\mathrm{R}$ & $05 / 24 / 04$ & 1300 & 47 & 113 & -- \\
\hline
\end{tabular}


Table 6. Analytical results for glyphosate, aminomethylphosphonic acid, and glufosinate in Toxic Substances Hydrology Program studies, 2001-06.— Continued

[Sample type: FB, field blank; FR, field replicate; R, regular; LD, laboratory duplicate; --, no data; <, less than; $\mu$ g/L, micrograms per liter]

\begin{tabular}{|c|c|c|c|c|c|c|c|}
\hline \multirow[b]{2}{*}{ Site number } & \multirow[b]{2}{*}{ Site location/soil core depth in inches (“) } & \multirow[b]{2}{*}{$\begin{array}{c}\text { Sam- } \\
\text { ple } \\
\text { type }\end{array}$} & \multirow[b]{2}{*}{$\begin{array}{c}\text { Date of } \\
\text { collection } \\
\text { (month/day/ } \\
\text { year) }\end{array}$} & \multirow[b]{2}{*}{$\begin{array}{l}\text { Col- } \\
\text { lec- } \\
\text { tion } \\
\text { time } \\
\text { (24- } \\
\text { hour) }\end{array}$} & \multicolumn{3}{|c|}{ Concentrations } \\
\hline & & & & & $\begin{array}{l}\text { Glypho- } \\
\text { sate } \\
\text { (pg/L) }\end{array}$ & $\begin{array}{l}\text { Amino- } \\
\text { meth- } \\
\text { ylphos- } \\
\text { phonic } \\
\text { acid } \\
\text { ( } \mu \mathrm{g} / \mathrm{L} \text { ) }\end{array}$ & $\begin{array}{c}\text { Glufos- } \\
\text { inate } \\
(\mu \mathrm{g} / \mathrm{L})\end{array}$ \\
\hline \multicolumn{8}{|c|}{ Sensitive environments study, 2004-06-Continued } \\
\hline \multicolumn{8}{|c|}{ Leary Weber Ditch Basin, Indiana (soils)_Continued } \\
\hline 395151085504530 & Vadose zone ACTVZ1-2A at Mohawk (North, 0-6") & LD & $05 / 24 / 04$ & 1300 & 69 & 85 & -- \\
\hline 395151085504530 & Vadose zone ACTVZ1-2A at Mohawk (North, 0-6”) & $\mathrm{R}$ & $05 / 24 / 04$ & 1301 & 78 & 116 & -- \\
\hline 395151085504530 & Vadose zone ACTVZ1-2A at Mohawk (North, 0-6”) & $\mathrm{R}$ & $05 / 24 / 04$ & 1302 & 67 & 208 & -- \\
\hline 395151085504530 & Vadose zone ACTVZ1-2A at Mohawk (North, 6-12”) & $\mathrm{R}$ & $05 / 24 / 04$ & 1315 & 6.0 & 22 & -- \\
\hline 395151085504530 & Vadose zone ACTVZ1-2A at Mohawk (North, 6-12”) & $\mathrm{R}$ & $05 / 24 / 04$ & 1316 & 3.0 & 21 & -- \\
\hline 395151085504530 & Vadose zone ACTVZ1-2A at Mohawk (North, 6-12”) & $\mathrm{R}$ & $05 / 24 / 04$ & 1317 & 1.0 & 13 & -- \\
\hline 395151085504530 & Vadose zone ACTVZ1-2A at Mohawk (North, 12-18”) & $\mathrm{R}$ & $05 / 24 / 04$ & 1330 & 1.0 & 2.0 & -- \\
\hline 395151085504530 & Vadose zone ACTVZ1-2A at Mohawk (North, 12-18”) & $\mathrm{R}$ & $05 / 24 / 04$ & 1331 & 15 & 21 & -- \\
\hline 395151085504530 & Vadose zone ACTVZ1-2A at Mohawk (North, 12-18”) & $\mathrm{R}$ & $05 / 24 / 04$ & 1332 & 1.0 & 3.0 & -- \\
\hline 395151085504530 & Vadose zone ACTVZ1-2A at Mohawk (North, 0-6") & $\mathrm{R}$ & 07/07/04 & 1300 & 85 & 162 & -- \\
\hline 395151085504530 & Vadose zone ACTVZ1-2A at Mohawk (North, 0-6”) & $\mathrm{R}$ & 07/07/04 & 1301 & 23 & 142 & -- \\
\hline 395151085504530 & Vadose zone ACTVZ1-2A at Mohawk (North, 0-6”) & $\mathrm{R}$ & 07/07/04 & 1302 & 90 & 169 & -- \\
\hline 395151085504530 & Vadose zone ACTVZ1-2A at Mohawk (North, 6-12”) & $\mathrm{R}$ & 07/07/04 & 1330 & 17 & 31 & -- \\
\hline 395151085504530 & Vadose zone ACTVZ1-2A at Mohawk (North, 6-12”) & $\mathrm{R}$ & 07/07/04 & 1331 & 2.2 & 13 & -- \\
\hline 395151085504530 & Vadose zone ACTVZ1-2A at Mohawk (North, 6-12”) & $\mathrm{R}$ & 07/07/04 & 1332 & 12 & 21 & -- \\
\hline 395151085504530 & Vadose zone ACTVZ1-2A at Mohawk (North, 12-18”) & $\mathrm{R}$ & 07/07/04 & 1400 & 2.5 & 9.8 & -- \\
\hline 395151085504530 & Vadose zone ACTVZ1-2A at Mohawk (North, 12-18”) & $\mathrm{R}$ & 07/07/04 & 1401 & 3.7 & 14 & -- \\
\hline 395151085504530 & Vadose zone ACTVZ1-2A at Mohawk (North, 12-18”) & $\mathrm{R}$ & 07/07/04 & 1402 & 6.1 & 12 & -- \\
\hline 39515108504530 & Vadose zone ACTVZ1-2A at Mohawk (North, 0-6") & $\mathrm{R}$ & $10 / 21 / 04$ & 0930 & 25 & 127 & $<1.0$ \\
\hline 39515108504530 & Vadose zone ACTVZ1-2A at Mohawk (North, 0-6”) & LD & $10 / 21 / 04$ & 0930 & 27 & 99 & $<1.0$ \\
\hline 395151085504530 & Vadose zone ACTVZ1-2A at Mohawk (North, 0-6”) & $\mathrm{R}$ & $10 / 21 / 04$ & 0931 & 60 & 477 & $<1.0$ \\
\hline 395151085504530 & Vadose zone ACTVZ1-2A at Mohawk (North, 0-6") & $\mathrm{R}$ & $10 / 21 / 04$ & 0932 & 144 & 956 & $<1.0$ \\
\hline 395151085504530 & Vadose zone ACTVZ1-2A at Mohawk (North, 6-12”) & $\mathrm{R}$ & $10 / 21 / 04$ & 0940 & $<1.0$ & 9.5 & $<1.0$ \\
\hline 395151085504530 & Vadose zone ACTVZ1-2A at Mohawk (North, 6-12”) & $\mathrm{LD}$ & $10 / 21 / 04$ & 0940 & $<1.0$ & 11 & $<1.0$ \\
\hline 395151085504530 & Vadose zone ACTVZ1-2A at Mohawk (North, 6-12”) & $\mathrm{R}$ & $10 / 21 / 04$ & 0941 & $<1.0$ & 1.9 & $<1.0$ \\
\hline 395151085504530 & Vadose zone ACTVZ1-2A at Mohawk (North, 6-12”) & $\mathrm{R}$ & $10 / 21 / 04$ & 0942 & $<1.0$ & 4.9 & 1.0 \\
\hline 395151085504530 & Vadose zone ACTVZ1-2A at Mohawk (North, 12-18”) & $\mathrm{R}$ & $10 / 21 / 04$ & 0950 & $<1.0$ & $<1.0$ & $<1.0$ \\
\hline 395151085504530 & Vadose zone ACTVZ1-2A at Mohawk (North, 12-18”) & $\mathrm{R}$ & $10 / 21 / 04$ & 0951 & $<1.0$ & $<1.0$ & $<1.0$ \\
\hline
\end{tabular}


Table 6. Analytical results for glyphosate, aminomethylphosphonic acid, and glufosinate in Toxic Substances Hydrology Program studies, 2001-06. Continued

[Sample type: FB, field blank; FR, field replicate; R, regular; LD, laboratory duplicate; --, no data; <, less than; $\mu \mathrm{g} / \mathrm{L}$, micrograms per liter]

\begin{tabular}{|c|c|c|c|c|c|c|c|}
\hline \multirow[b]{2}{*}{ Site number } & \multirow[b]{2}{*}{ Site location/soil core depth in inches (") } & \multirow[b]{2}{*}{$\begin{array}{c}\text { Sam- } \\
\text { ple } \\
\text { type }\end{array}$} & \multirow[b]{2}{*}{$\begin{array}{c}\text { Date of } \\
\text { collection } \\
\text { (month/day/ } \\
\text { year) }\end{array}$} & \multirow[b]{2}{*}{$\begin{array}{l}\text { Col- } \\
\text { lec- } \\
\text { tion } \\
\text { time } \\
(24- \\
\text { hour) }\end{array}$} & \multicolumn{3}{|c|}{ Concentrations } \\
\hline & & & & & $\begin{array}{c}\text { Glypho- } \\
\text { sate } \\
\text { ( } \mu \mathrm{g} / \mathrm{L})\end{array}$ & $\begin{array}{l}\text { Amino- } \\
\text { meth- } \\
\text { ylphos- } \\
\text { phonic } \\
\text { acid } \\
\text { ( } \mu \mathrm{g} / \mathrm{L})\end{array}$ & $\begin{array}{c}\text { Glufos- } \\
\text { inate } \\
(\mu \mathrm{g} / \mathrm{L})\end{array}$ \\
\hline \multicolumn{8}{|c|}{ Sensitive environments study, 2004-06-Continued } \\
\hline \multicolumn{8}{|c|}{ Leary Weber Ditch Basin, Indiana (soils)_Continued } \\
\hline 395151085504530 & Vadose zone ACTVZ1-2A at Mohawk (North, 12-18”) & $\mathrm{R}$ & $10 / 21 / 04$ & 0952 & $<1.0$ & $<1.0$ & $<1.0$ \\
\hline 395151085504530 & Vadose zone ACTVZ1-2A at Mohawk (North, 0-6") & $\mathrm{R}$ & $04 / 19 / 05$ & 1030 & 18 & 75 & $<1.0$ \\
\hline 395151085504530 & Vadose zone ACTVZ1-2A at Mohawk (North, 0-6") & $\mathrm{R}$ & $04 / 19 / 05$ & 1040 & 8.0 & 62 & $<1.0$ \\
\hline 395151085504530 & Vadose zone ACTVZ1-2A at Mohawk (North, 0-6") & $\mathrm{R}$ & $04 / 19 / 05$ & 1041 & $<1.0$ & 6.9 & $<1.0$ \\
\hline 395151085504530 & Vadose zone ACTVZ1-2A at Mohawk (North, 6-12”) & $\mathrm{R}$ & $04 / 19 / 05$ & 1031 & 1.0 & 4.1 & $<1.0$ \\
\hline 395151085504530 & Vadose zone ACTVZ1-2A at Mohawk (North, 6-12”) & $\mathrm{R}$ & $04 / 19 / 05$ & 1042 & $<1.0$ & $<1.0$ & $<1.0$ \\
\hline 395151085504530 & Vadose zone ACTVZ1-2A at Mohawk (North, 6-12”) & $\mathrm{R}$ & $04 / 19 / 05$ & 1050 & 2.6 & 33 & $<1.0$ \\
\hline 395151085504530 & Vadose zone ACTVZ1-2A at Mohawk (North, 12-18") & $\mathrm{R}$ & $04 / 19 / 05$ & 1032 & $<1.0$ & 1.3 & $<1.0$ \\
\hline 395151085504530 & Vadose zone ACTVZ1-2A at Mohawk (North, 12-18”) & $\mathrm{R}$ & $04 / 19 / 05$ & 1051 & $<1.0$ & 9.3 & $<1.0$ \\
\hline 395151085504530 & Vadose zone ACTVZ1-2A at Mohawk (North, 12-18”) & $\mathrm{R}$ & $04 / 19 / 05$ & 1052 & $<1.0$ & $<1.0$ & $<1.0$ \\
\hline 395151085504530 & Vadose zone ACTVZ1-2A at Mohawk (North, 0-6") & $\mathrm{R}$ & $05 / 25 / 05$ & 0900 & 36 & 148 & $<1.0$ \\
\hline 395151085504530 & Vadose zone ACTVZ1-2A at Mohawk (North, 0-6”) & $\mathrm{R}$ & $05 / 25 / 05$ & 0910 & 9.7 & 119 & $<1.0$ \\
\hline 395151085504530 & Vadose zone ACTVZ1-2A at Mohawk (North, 0-6") & $\mathrm{R}$ & $05 / 25 / 05$ & 0920 & 28 & 58 & $<1.0$ \\
\hline 395151085504530 & Vadose zone ACTVZ1-2A at Mohawk (North, 6-12”) & $\mathrm{R}$ & $05 / 25 / 05$ & 0901 & 3.0 & 12 & $<1.0$ \\
\hline 395151085504530 & Vadose zone ACTVZ1-2A at Mohawk (North, 6-12”) & $\mathrm{R}$ & $05 / 25 / 05$ & 0911 & $<1.0$ & 2.0 & $<1.0$ \\
\hline 395151085504530 & Vadose zone ACTVZ1-2A at Mohawk (North, 6-12”) & $\mathrm{LD}$ & $05 / 25 / 05$ & 0911 & $<1.0$ & 2.3 & $<1.0$ \\
\hline 395151085504530 & Vadose zone ACTVZ1-2A at Mohawk (North, 6-12”) & $\mathrm{R}$ & $05 / 25 / 05$ & 0921 & $<1.0$ & 1.6 & $<1.0$ \\
\hline 395151085504530 & Vadose zone ACTVZ1-2A at Mohawk (North, 12-18") & $\mathrm{R}$ & $05 / 25 / 05$ & 0902 & 1.3 & 8.4 & $<1.0$ \\
\hline 395151085504530 & Vadose zone ACTVZ1-2A at Mohawk (North, 12-18”) & $\mathrm{R}$ & $05 / 25 / 05$ & 0912 & $<1.0$ & $<1.0$ & $<1.0$ \\
\hline 395151085504530 & Vadose zone ACTVZ1-2A at Mohawk (North-12-18”) & $\mathrm{R}$ & $05 / 25 / 05$ & 0922 & $<1.0$ & $<1.0$ & $<1.0$ \\
\hline 395120085502401 & Vadose ACT: Overland Flow at Mohawk (0-6”) & $\mathrm{R}$ & 07/07/04 & 1100 & 476 & 341 & -- \\
\hline 395120085502401 & Vadose ACT: Overland Flow at Mohawk (0-6") & $\mathrm{R}$ & 07/07/04 & 1101 & 103 & 127 & -- \\
\hline 395120085502401 & Vadose ACT: Overland Flow at Mohawk (0-6") & $\mathrm{R}$ & 07/07/04 & 1102 & 88 & 150 & -- \\
\hline 395120085502401 & Vadose ACT: Overland Flow at Mohawk (6-12”) & $\mathrm{R}$ & 07/07/04 & 1130 & 24 & 21 & -- \\
\hline 395120085502401 & Vadose ACT: Overland Flow at Mohawk (6-12”) & $\mathrm{R}$ & 07/07/04 & 1131 & 4.4 & 9.4 & -- \\
\hline 395120085502401 & Vadose ACT: Overland Flow at Mohawk (6-12”) & $\mathrm{R}$ & 07/07/04 & 1132 & 40 & 54 & -- \\
\hline 395120085502401 & Vadose ACT: Overland Flow at Mohawk (12-18”) & $\mathrm{R}$ & 07/07/04 & 1200 & 19 & 14 & -- \\
\hline 395120085502401 & Vadose ACT: Overland Flow at Mohawk (12-18”) & $\mathrm{R}$ & 07/07/04 & 1201 & 17 & 25 & -- \\
\hline
\end{tabular}


Table 6. Analytical results for glyphosate, aminomethylphosphonic acid, and glufosinate in Toxic Substances Hydrology Program studies, 2001-06.Continued

[Sample type: FB, field blank; FR, field replicate; R, regular; LD, laboratory duplicate; --, no data; <, less than; $\mu \mathrm{g} / \mathrm{L}$, micrograms per liter]

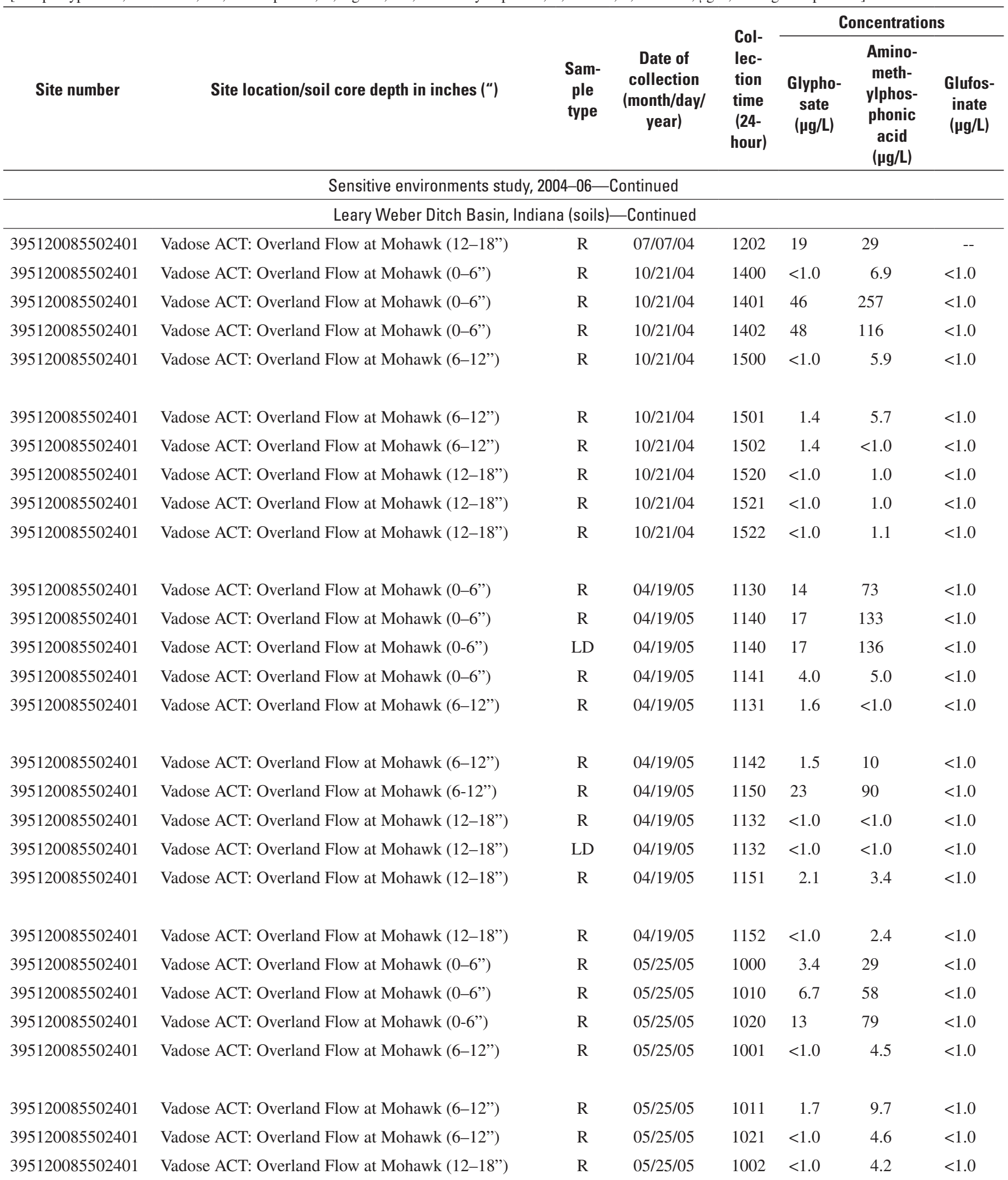


Table 6. Analytical results for glyphosate, aminomethylphosphonic acid, and glufosinate in Toxic Substances Hydrology Program studies, 2001-06. Continued

[Sample type: FB, field blank; FR, field replicate; R, regular; LD, laboratory duplicate; --, no data; <, less than; $\mu \mathrm{g} / \mathrm{L}$, micrograms per liter]

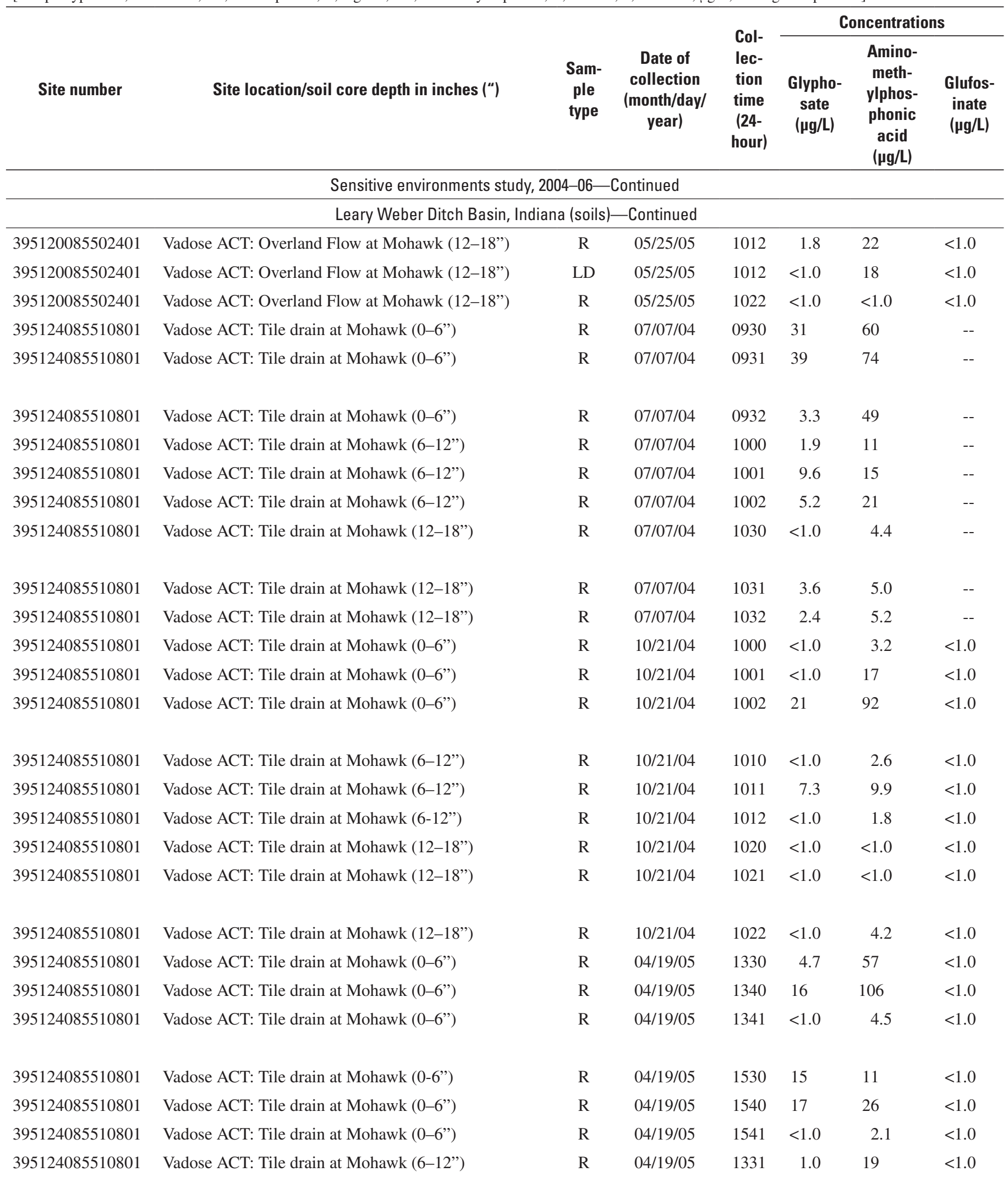


Table 6. Analytical results for glyphosate, aminomethylphosphonic acid, and glufosinate in Toxic Substances Hydrology Program studies, 2001-06. Continued

[Sample type: FB, field blank; FR, field replicate; R, regular; LD, laboratory duplicate; --, no data; <, less than; $\mu$ g/L, micrograms per liter]

\begin{tabular}{|c|c|c|c|c|c|c|c|}
\hline \multirow[b]{2}{*}{ Site number } & \multirow[b]{2}{*}{ Site location/soil core depth in inches (“) } & \multirow[b]{2}{*}{$\begin{array}{l}\text { Sam- } \\
\text { ple } \\
\text { type }\end{array}$} & \multirow[b]{2}{*}{$\begin{array}{c}\text { Date of } \\
\text { collection } \\
\text { (month/day/ } \\
\text { year) }\end{array}$} & \multirow[b]{2}{*}{$\begin{array}{c}\text { Col- } \\
\text { lec- } \\
\text { tion } \\
\text { time } \\
(24- \\
\text { hour) }\end{array}$} & \multicolumn{3}{|c|}{ Concentrations } \\
\hline & & & & & $\begin{array}{c}\text { Glypho- } \\
\text { sate } \\
\text { ( } \mu \mathrm{g} / \mathrm{L})\end{array}$ & $\begin{array}{l}\text { Amino- } \\
\text { meth- } \\
\text { ylphos- } \\
\text { phonic } \\
\text { acid } \\
\text { ( } \mathrm{g} / \mathrm{L})\end{array}$ & $\begin{array}{c}\text { Glufos- } \\
\text { inate } \\
\text { ( } \mu \mathrm{g} / \mathrm{L})\end{array}$ \\
\hline \multicolumn{8}{|c|}{ Sensitive environments study, 2004-06-Continued } \\
\hline \multicolumn{8}{|c|}{ Leary Weber Ditch Basin, Indiana (soils)—Continued } \\
\hline 395124085510801 & Vadose ACT: Tile drain at Mohawk (6-12") & $\mathrm{R}$ & $04 / 19 / 05$ & 1342 & $<1.0$ & 2.5 & $<1.0$ \\
\hline 395124085510801 & Vadose ACT: Tile drain at Mohawk (6-12”) & $\mathrm{R}$ & $04 / 19 / 05$ & 1350 & 7.0 & 60 & $<1.0$ \\
\hline 395124085510801 & Vadose ACT: Tile drain at Mohawk (6-12") & $\mathrm{R}$ & $04 / 19 / 05$ & 1531 & $<1.0$ & $<1.0$ & $<1.0$ \\
\hline 395124085510801 & Vadose ACT: Tile drain at Mohawk (6-12") & $\mathrm{R}$ & $04 / 19 / 05$ & 1542 & $<1.0$ & $<1.0$ & $<1.0$ \\
\hline 395124085510801 & Vadose ACT: Tile drain at Mohawk (6-12") & $\mathrm{R}$ & $04 / 19 / 05$ & 1550 & 17 & $<1.0$ & $<1.0$ \\
\hline 395124085510801 & Vadose ACT: Tile drain at Mohawk (12-18”) & $\mathrm{R}$ & $04 / 19 / 05$ & 1332 & $<1.0$ & 6.6 & $<1.0$ \\
\hline 395124085510801 & Vadose ACT: Tile drain at Mohawk (12-18”) & $\mathrm{R}$ & $04 / 19 / 05$ & 1351 & $<1.0$ & 2.6 & $<1.0$ \\
\hline 395124085510801 & Vadose ACT: Tile drain at Mohawk (12-18”) & $\mathrm{LD}$ & $04 / 19 / 05$ & 1351 & $<1.0$ & $<1.0$ & $<1.0$ \\
\hline 395124085510801 & Vadose ACT: Tile drain at Mohawk (12-18”) & $\mathrm{R}$ & $04 / 19 / 05$ & 1352 & $<1.0$ & $<1.0$ & $<1.0$ \\
\hline 395124085510801 & Vadose ACT: Tile drain at Mohawk (12-18") & $\mathrm{R}$ & $04 / 19 / 05$ & 1532 & $<1.0$ & 1.1 & $<1.0$ \\
\hline 395124085510801 & Vadose ACT: Tile drain at Mohawk (12-18”) & $\mathrm{R}$ & $04 / 19 / 05$ & 1551 & $<1.0$ & $<1.0$ & $<1.0$ \\
\hline 395124085510801 & Vadose ACT: Tile drain at Mohawk (12-18”) & $\mathrm{R}$ & $04 / 19 / 05$ & 1552 & $<1.0$ & $<1.0$ & $<1.0$ \\
\hline 395124085510801 & Vadose ACT: Tile drain at Mohawk (0-6”) & $\mathrm{R}$ & $05 / 25 / 05$ & 1200 & 6.6 & 5.5 & $<1.0$ \\
\hline 395124085510801 & Vadose ACT: Tile drain at Mohawk (0-6") & $\mathrm{R}$ & $05 / 25 / 05$ & 1210 & 9.2 & 1.8 & $<1.0$ \\
\hline 395124085510801 & Vadose ACT: Tile drain at Mohawk (0-6") & $\mathrm{LD}$ & $05 / 25 / 05$ & 1210 & 8.3 & 2.5 & $<1.0$ \\
\hline 395124085510801 & Vadose ACT: Tile drain at Mohawk (0-6") & $\mathrm{R}$ & $05 / 25 / 05$ & 1220 & 17 & 3.7 & $<1.0$ \\
\hline 395124085510801 & Vadose ACT: Tile drain at Mohawk (0-6") & $\mathrm{R}$ & $05 / 25 / 05$ & 1300 & 10 & 104 & $<1.0$ \\
\hline 395124085510801 & Vadose ACT: Tile drain at Mohawk (0-6") & $\mathrm{R}$ & $05 / 25 / 05$ & 1310 & 6.9 & 65 & $<1.0$ \\
\hline 395124085510801 & Vadose ACT: Tile drain at Mohawk (0-6") & $\mathrm{R}$ & $05 / 25 / 05$ & 1320 & $<1.0$ & 11 & $<1.0$ \\
\hline 395124085510801 & Vadose ACT: Tile drain at Mohawk (6-12") & $\mathrm{R}$ & $05 / 25 / 05$ & 1201 & $<1.0$ & $<1.0$ & $<1.0$ \\
\hline 395124085510801 & Vadose ACT: Tile drain at Mohawk (6-12") & $\mathrm{R}$ & $05 / 25 / 05$ & 1211 & 1.7 & $<1.0$ & $<1.0$ \\
\hline 395124085510801 & Vadose ACT: Tile drain at Mohawk (6-12”) & $\mathrm{R}$ & $05 / 25 / 05$ & 1221 & 1.0 & $<1.0$ & $<1.0$ \\
\hline 395124085510801 & Vadose ACT: Tile drain at Mohawk (6-12”) & $\mathrm{R}$ & $05 / 25 / 05$ & 1301 & $<1.0$ & 13 & $<1.0$ \\
\hline 395124085510801 & Vadose ACT: Tile drain at Mohawk (6-12”) & $\mathrm{R}$ & $05 / 25 / 05$ & 1311 & $<1.0$ & 4.7 & $<1.0$ \\
\hline 395124085510801 & Vadose ACT: Tile drain at Mohawk (6-12”) & $\mathrm{LD}$ & $05 / 25 / 05$ & 1311 & $<1.0$ & 3.5 & $<1.0$ \\
\hline 395124085510801 & Vadose ACT: Tile drain at Mohawk (6-12”) & $\mathrm{R}$ & $05 / 25 / 05$ & 1321 & $<1.0$ & $<1.0$ & $<1.0$ \\
\hline 395124085510801 & Vadose ACT: Tile drain at Mohawk (12-18”) & $\mathrm{R}$ & $05 / 25 / 05$ & 1202 & 4.1 & $<1.0$ & $<1.0$ \\
\hline 395124085510801 & Vadose ACT: Tile drain at Mohawk (12-18”) & $\mathrm{R}$ & $05 / 25 / 05$ & 1212 & 7.1 & $<1.0$ & $<1.0$ \\
\hline
\end{tabular}


Table 6. Analytical results for glyphosate, aminomethylphosphonic acid, and glufosinate in Toxic Substances Hydrology Program studies, 2001-06. Continued

[Sample type: FB, field blank; FR, field replicate; R, regular; LD, laboratory duplicate; --, no data; <, less than; $\mu \mathrm{g} / \mathrm{L}$, micrograms per liter]

\begin{tabular}{|c|c|c|c|c|c|c|c|}
\hline \multirow[b]{2}{*}{ Site number } & \multirow[b]{2}{*}{ Site location/soil core depth in inches (“) } & \multirow[b]{2}{*}{$\begin{array}{c}\text { Sam- } \\
\text { ple } \\
\text { type }\end{array}$} & \multirow[b]{2}{*}{$\begin{array}{c}\text { Date of } \\
\text { collection } \\
\text { (month/day/ } \\
\text { year) }\end{array}$} & \multirow[b]{2}{*}{$\begin{array}{l}\text { Col- } \\
\text { lec- } \\
\text { tion } \\
\text { time } \\
\text { (24- } \\
\text { hour) }\end{array}$} & \multicolumn{3}{|c|}{ Concentrations } \\
\hline & & & & & $\begin{array}{c}\text { Glypho- } \\
\text { sate } \\
\text { ( } \mu \mathrm{g} / \mathrm{L})\end{array}$ & 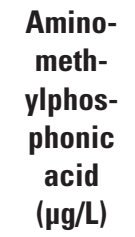 & $\begin{array}{c}\text { Glufos- } \\
\text { inate } \\
(\mu \mathrm{g} / \mathrm{L})\end{array}$ \\
\hline \multicolumn{8}{|c|}{ Sensitive environments study, 2004-06-Continued } \\
\hline \multicolumn{8}{|c|}{ Leary Weber Ditch Basin, Indiana (soils)_Continued } \\
\hline 395124085510801 & Vadose ACT: Tile drain at Mohawk (12-18”) & $\mathrm{R}$ & $05 / 25 / 05$ & 1222 & 3.5 & $<1.0$ & $<1.0$ \\
\hline 395124085510801 & Vadose ACT: Tile drain at Mohawk (12-18”) & $\mathrm{R}$ & $05 / 25 / 05$ & 1302 & $<1.0$ & 1.9 & $<1.0$ \\
\hline 395124085510801 & Vadose ACT: Tile drain at Mohawk (12-18”) & $\mathrm{R}$ & $05 / 25 / 05$ & 1312 & $<1.0$ & $<1.0$ & $<1.0$ \\
\hline 395124085510801 & Vadose ACT: Tile drain at Mohawk (12-18”) & $\mathrm{R}$ & $05 / 25 / 05$ & 1322 & $<1.0$ & 1.7 & $<1.0$ \\
\hline 03361638 & Leary Weber Ditch at Mohawk (0-6”) & $\mathrm{R}$ & $04 / 19 / 05$ & 1730 & 2.2 & 4.0 & $<1.0$ \\
\hline 03361638 & Leary Weber Ditch at Mohawk (0-6”) & FR & $04 / 19 / 05$ & 1740 & $<1.0$ & $<1.0$ & $<1.0$ \\
\hline 03361638 & Leary Weber Ditch at Mohawk (0-6") & FR & $04 / 19 / 05$ & 1750 & $<1.0$ & $<1.0$ & $<1.0$ \\
\hline 03361638 & Leary Weber Ditch at Mohawk (0-6”) & FR & $04 / 19 / 05$ & 1751 & $<1.0$ & $<1.0$ & $<1.0$ \\
\hline 03361638 & Leary Weber Ditch at Mohawk (0-6") & $\mathrm{R}$ & $05 / 25 / 05$ & 1100 & $<1.0$ & $<1.0$ & $<1.0$ \\
\hline 03361638 & Leary Weber Ditch at Mohawk (0-6") & FR & $05 / 25 / 05$ & 1110 & $<1.0$ & $<1.0$ & $<1.0$ \\
\hline 03361638 & Leary Weber Ditch at Mohawk (0-6") & FR & $05 / 25 / 05$ & 1120 & $<1.0$ & $<1.0$ & $<1.0$ \\
\hline
\end{tabular}


Prepared by Lawrence Publishing Service Center. Edited by Lanna Combs.

Illustrations and cover design by Jeff Hartley.

Layout and design by Kristi Hartley.

For more information concerning the research described in this report, contact:

U.S. Geological Survey

4821 Quail Crest Place

Lawrence, KS 66049

(785) 842-9909

http://ks.water.usgs.gov 

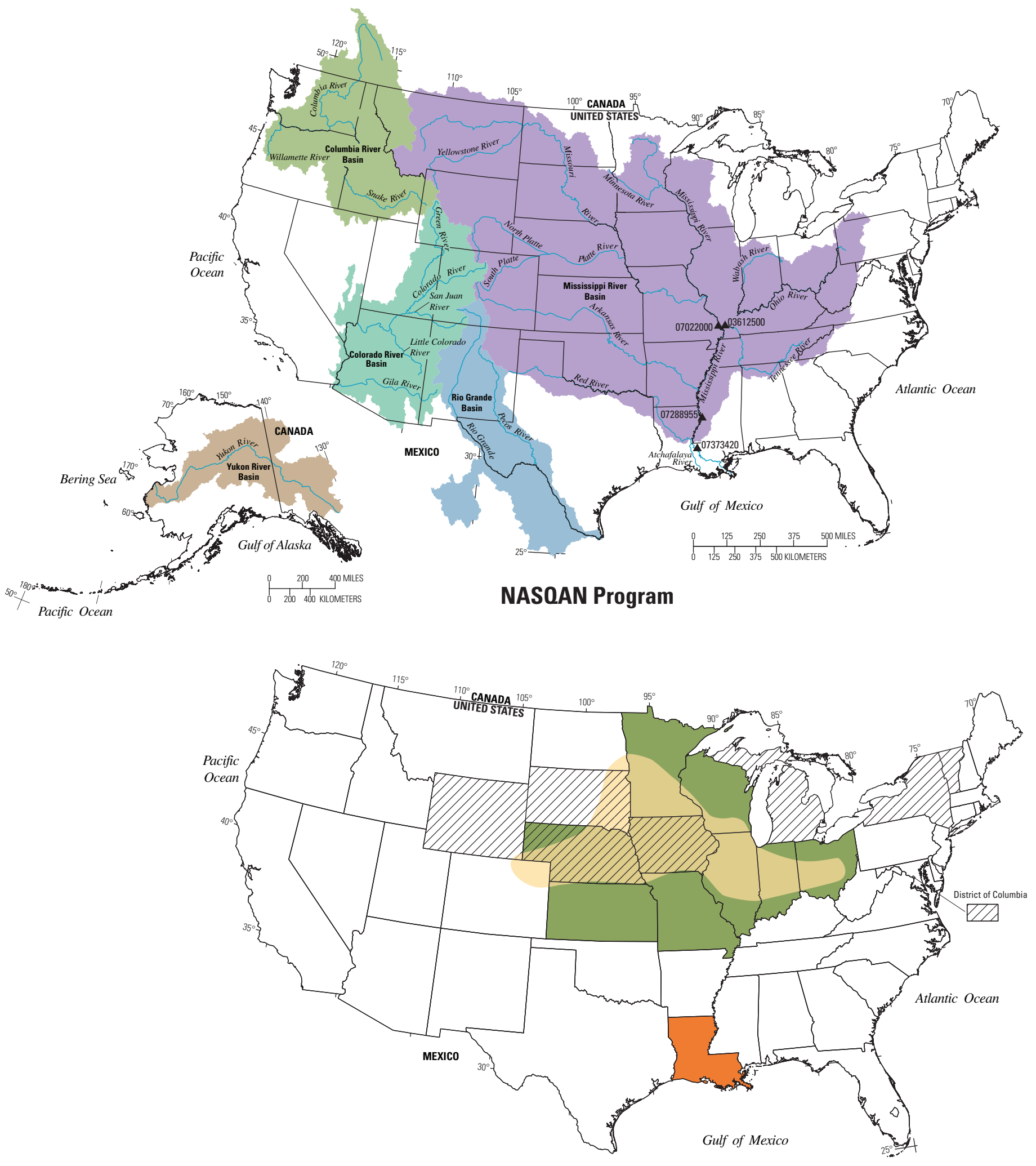

Toxic Substances Program

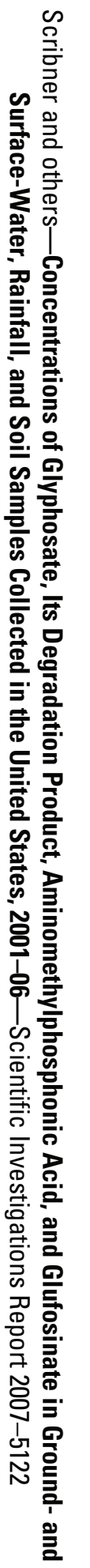

